STATE OF ALASKA

DEPARTMENT OF NATURAL RESOURCES

DIVISION OF GEOLOGICAL AND GEOPHYSICAL SURVEYS

\author{
Robert E. LaResche - Commissioner \\ Ross G. Schaff - State Geologist
}

May 1977

This is a preliminary publication of the Alaska Division of Geological and Geophysical Surveys and as such has not received final editing ond review. It may bc quoted only through permission of the author and State Geologist. The author will appreciate candid comments on the accuracy of the data, and welcomes suggestions that will improve the report.

\author{
Alaska Open-File Report 109 \\ INVESTIGATION OF ALASKA'S \\ URANIUM POTENTIAL
}

By G.R. Eakins, B.K. Jones and R.B. Forbes 


\section{INVESTIGATION OF ALASKA'S URANIUM POTENTIAL}

Part 1 - Reconnaissance Program. West-Central Alaska and Copper River Basin By Gilbert R. Eakins

Originally prepared as a Summary Report for the U.S. Energy Research and Development Administration-Grand Junction Officeunder Contract AT(05-1)-1639 


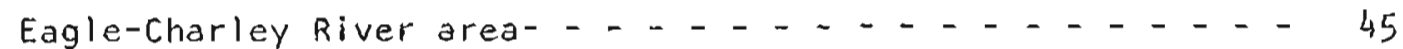

Stream-sediment sampling - - - - - - - - - - - 46

Water sampling - - - - - - - - - - - - - 47

Bedrock sampling - - - - - - - - - - - - - - 47

Aerial radiometric survey- - - - - - - - - - - - 48

Ground radiometric survey- - - - - - - - - - - 48

Suggestions for exploration - - - - - - - - - - 48

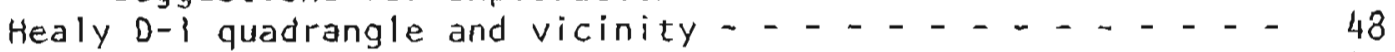

Stream-sediment sampling - - - - - - - - - - - - 49

Suggestions for exploration- - - - - - - - - - 50

Conclusions - - - - - - - - - - - - - - - - - - - 50

References - - - - - - - - - - - - - - - - - - 52

APPENDIX A-Stream-sediment-, rock , and water-sample analyses

with radiometric data - - - - - - - - - - - - - - A-l

APPENDIX B-Histograms- - - - - - - - - - - - - - - - - B B

APPENDIX C-Correlation matrix- - - - - - - - - - - - - C-1

APPENDIX D-Determination of thorium, uranium, and potassium

oxide in stream sediments and rocks - - - - - - - - - - D-1

APPEHDIX E General geology of areas investigated - - - - - - - E-l

ILLUSTRATIONS

Page

Plate 1. Western Candle quadrangle: Sample locations, generalized geology, and aerial radiometric survey data - . - . . . . . - - . . . - - In pocker

2. Eastern Solomon and southeastern Bendeleben quadrangles: Sample locations, generalized geology, and aerial radiometric data - - . - . - . - In pocket

3. Selawik Hills area: Sample locations, generalized geology, and aerial radiometric data- - - - - - In pocket

4. Zane Hills and Purcell Mountains area: Sample locations, generalized geology, and aerial radiometric data - - - . - . - . - - - - In pocket

5. Eastern Talkeetna Mountains and southwestern Gulkana quadrangles: Sample locations, generalized geology, and aerial radiometric data- - - - - - In pocket

6. Southern and northwestern McCarthy quadrangles: Sample locations and generalized geology- - - . - In pocket

7. Eastern and northern Valdez quadrangles: Sample locations and generalized geology - . - . - . - In pocket

8. Eagle and Charley River area: Sample locations and generalized geology _. . . . . . - - - In pocket

9. Healy D-l quadrangle and vicinity: Sample locations and generalized geology - . . - . - . - - In pocket

10. Central Mt. Hayes quadrangle (Richardson Highway): Sample locations and generalized geology- . . . . in pocket

Figure 1. Index map of areas investigated for uranium by DGGS,

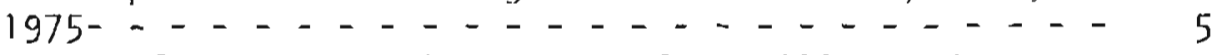

2. Plutons of west-central Alaska; after Miller and Bunker, 1975- - - - - - - - - - - - - - 12

3. Ground radiometric survey of the Granite Mountain area, Candle $\mathrm{B}-5$ quadrangle - - - $_{-}-$- $_{-}-$- $_{-}$ 
Figure 4. Ground radiometric survey of the Hunter Creek pluton, Candle $\mathrm{C}-5$ and $\mathrm{C}-6$ quadrangles $\ldots \ldots$

5. Ground radiometric survey of the northern part of the Hunter Creek pluton, Candle D-5 quadrangle- - 13

6. Generalized geology of the Darby Mountains area- - - - 22

7. Location of mineralized areas and analyzed samples referred to in table $3 \ldots \ldots 24$

8. Ground radiometric survey of the southern Darby Mountains area, Solomon $8-2$ and $C-2$ quadrangles- - - 27

9. Ground radiometric survey of the northern Darby Mountains - - . . . . . . - . . - - 28

10. Ground radiometric survey of the Selawik Hills, Selawik A-3 and A-4 quadrangles- _ . . . . . 32

11. Ground radiometric survey of the western Selawik Hills, Selawik B-3 quadrangle _ . . . . . . . . - 33

12. Ground radiometric survey, Selawik Hills-Inland Lake area, Selawik A-2 and B-2 quadrangles- - . - . 34

13. Ground radiometric survey of the southern Zane Hills, Hughes A-6 and Shungnak A-l quadrangles- - - - - 37

14. Ground radiometric survey of the northern Zane Hills, Hughes B-6 and Shungnak B- 1 quadrangles - - . - - 38

15. Ground radiometric survey of the Purcell Mountains, Shungnak A-3, A-4, B-3, and B-4 quadrangles- - - 39

16. Index map of the Copper River basin and Chitina River valley sample areas, south-central Alaska- - - - - 41

17. Index map of the Eagle-Charley River sample area, eastcentral Alaska - . . . . . . . . . . . . 47

18. Index map of the Healy D-I sample area, north flank of the central Alaska Range- . . . . . . . 49

\section{TABLES}

Table 1. Mineral composition of sample SEIR - - - - - - - 14

2. Mineral composition of sample 5E4R - - - - . - 15

3. Uranium and thorium analyses of samples from the Kachauik pluton- - - - - - - - - - - - 26

4. Sample locations, Fagle-Charley River area - . . - 45 
Two types of analyses for uranium in the stream-sediment samples are presented. One split of the stream-sediment samples was sent to Resource Associates of Alaska's laboratory, where they were analyzed for $U$ by the fluormetric method after acid extraction. This is the method employed for $U$ by most laboratories. The completeness of uranium extraction unavoidably varies with the particular type of uranium-bearing minerals present and possibly with the amount of iron, manganese, and carbonaceous material present, which can interfere with the extraction. Analyses by the method are shown in the text and appendix columns with the designation "RAA."

Splits of most of the stream-sediment samples were also analyzed by Los Almos Scientific Laboratory's (LASL) under the ir ERDA-sponsored Hydrochemical and Stream-sedinent Reconnaissance Program. Analyses at LASL were performed by the delayed-neutron counting method, which gives total uranium. This method can be used only if a reactor is available. Samples analyzed by this method are prefaced by the designation "LASL."

To provide as much information as possible and to illustrate what can be expected by the two different analytical methods, results of both are tabulated in the tables of analyses. It should be emphasized that the chemical analyses were monitored and frequently checked. Differences such as those shown between the chemical-fluormetric method and delayedneutron counting, according to LASL, are normal and to be expected. 


\section{ABSTRACT}

The Alaska Division of Geological and Geophysical Surveys (DGGS), under EROA contract AT(05-1)-1639, conducted a 6-week reconnaissance program in west-central Alaska and in the Copper River basin-Chitina River valley area to aid in determining the uranium potential of the state. Division personnel also submitted samples from the Healy, Eagle, and Charley River quadrangles.

DGGS personnel collected 916 stream-sediment samples and 427 bedrock samples for uranium, thorium, and potassium oxide determinations, and 565 water samples for uranium analyses. A statistical analys is of the determinations was made using a computer at the University of Alaska. The means, thresholds, anomalies, and $U: T h$ ratios were calculated for eight separate regions. A complete tabulation of the analyses is found in appendix $A$; anomalous values are indicated on the maps and in the tables.

A set of maps was constructed combining sample locations, aerial radiometric survey data, and the general geology. I sorad maps showing radiometric values determined by hand-carried scintillometers on the ground are included for the areas investigated in west-central Alaska.

The alkaline plutonic rocks in the west-central part of the state contain unusual amounts of uranium and thorium and are believed to have a potential for commercial uranium deposits. Stream-sediment samples from this region contained up to 111 ppm uranium, 150 ppm thorium, and 7.3 percent potassium oxide. Bedrock samples contained as much as 258 ppm uranium, $290 \mathrm{ppm}$ thorium, and 17.19 percent potassium oxide. The $U$ content of stream sediments was often higher than that of the surrounding bedrock, indicating concentrating of $U$ in the streams.

Water samples were found to generally be very low in uranium, but several anomalies were determined. The highest uranium content in the water samples was $3.95 \mathrm{ppb}$, obtained from a stream in the Zane Hills.

Analyses of sediments, bedrock, and water samples from the Copper River basin-Chitina River valley area, the Healy area, and the EagleCharley River area yielded disappointingiy low values for $U$. The maximum $U$ content of stream sediments was $10 \mathrm{ppm}$, rocks $2.5 \mathrm{ppm}$, and water $3.5 \mathrm{ppb}$. More detailed investigations in most of these areas, however, is recommended.

Petrographic studies of the plutonic rocks in west-central Alaska were performed by Dr. R.B. Forbes and B.K. Jones (part II of this report), who found a correlation of certain minerals with $U$ and Th within individual plutons, but no mineralogic guides to $U$ and Th that could be applied overall in the region. A limited number of calculations showed that nepheline syenite contained the most $U$ (mean $12.83 \mathrm{ppm}$ ) of the seven most common igneous rock types. Granite was second $(10.38 \mathrm{ppm} \mathrm{U).} \mathrm{The}$ highest concentrations of $U$ were found in alkalic dikes in the Darby Mountains and Selawik Hills, and in a broad zone in the Selawik Lake complex. 
Radiometric readings taken with hand-carried scintillometers at most sample sites and other locations yielded impressive values in the plutonic rocks of west-central Alaska. Backgrounds were commonly 200 to 400 counts per second (cps). The maximum was 2,000 cps over a dike in the Selawik Hills. In contrast with the plutonic belt in the western part of the state, radiometric readings obtained in the Copper River basin-Chitina River valley area were very low. The maximum reading in a single point was $160 \mathrm{cps}$, and the usual values were under $60 \mathrm{cps}$.

Although specific targets for drilling were not defined, the DGGS party concluded that all the plutons of west-central Alaska warrant detailed examination for potential uranium deposits. The Cretaceous sediments in the upper part of the chitina valley appear to deserve further investigation. Too little work was done in the Eagle-Charley River area to make any judgment, but the presence of a thick sequence of rocks ranging in age from Precambrian to Tertiary will no doubt be studied furcher by industry. Sampling results in the Healy D-I quadrangle were not encouraging, but farther west the belt of Tertiary coalbearing rocks and granite intrusives has produced uranium anamalies and is being explored by industry.

Anomalous values of the $U$, Th, and $\mathrm{K}_{2} \mathrm{O}$, and radiometric measurements are discussed for the separate areas, and suggestions are offered for exploration. A combination of all uranium exploration techniques is needed to locate potential uranium deposits in Alaska. For example, it was frequently found that only one type of sample (striam sediment, bedrock, or water) would show an anomally at a particular location, whereas the others did not. Also, correlations between aerial and ground radiometric surveys and geochemical surveys were often lackinn, indicating that each method may or may not be effective, depending on local conditions.

\section{ACKNOWLEDGMENTS}

The author wishes to acknowledge the assistance of others who, in many cases, contributed a great amount of time and effort. to certain phases of this study.

Cheri L. Carver, field assistant, performed the keypunching and computer work to obtain the statistical analyses of the sample determinations and assembled the data into useful form for the report. In addition, she was a dedicated assistant in the field, collecting and handling the saniples.

Dr. Daniel 8. Hawkins, professor and head of the Geology Department: at the University of Alaska, generously gave his time in writing the computer program and arranging for use of the University computer.

Dr. Robert R. Sharp, Jr., head of the hydrogeochemical and streamsediment project at the Los Alamos Scientific Laboratory, and his group performed uranium analyses of the water samples and splits of the streamsediment samples.

Henry S. Potworowski, laboratory supervisor at the OGGS laboratory, and his staff made $U$, Th, and $\mathrm{K}_{2} \mathrm{O}$ determinations, an essential part of this project. Numerous problems related to the analyses and equipment were worked out by these people. Namok Veach, assayer-chemist, performed some mineral analyses and identifications. 
Ann Schell, cartographer for the DGGS, spent many hours drafting the maps and figures.

Other members of the DGGS who contributed samples or assisted in map compilation include geologists Wyatt G. Gilbert, Mitchell W. Henning, Robert M. Kline, Thomas K. Bundtzen, and Patrick L. Dobey; and geological assistants Jeffrey $T$. KI ine and Jeffrey A. Morehouse.

Appreciation is due UV Industries and Jack $F$ isher, camp manager, for their hospitality at the Hogatza dredge mining camp.

\section{INTRODUCTION}

Reconnaissance sampling of stream sediments, water, and bedrock was conducted during 1975 in widespread areas of Alaska as a part of the National Uranium Resource Evaluation (NURE) program. The Alaska project was conducted by the Alaska State Division of Geological and Geophysical Surveys (DGGS) under a contract with the U.S. Energy Research and Development Administration (ERDA), who is the administrator of the NURE program. The sampling program is referred to as phase 11 by ERDA. Phase I consisted of a summary of published and unpublished geologica\} data and uranium investigations within the state and construction of a 1:1,000,000scale map of Alaska showing the distribution and classification of the alkaline intrusive rocks (Eakins and Forbes, 1975). The primary objective of phase 11 was to collect geochemical and radiometric data to aid industry in the search for new uranium deposits. Alaska is presently of particular interest because it is a large region that is still generally untested for uranium. The correlations between the $U$, $T h$, and $K_{2} O$ contents of water, stream sediments, and bedrock and radiometric surveys will help determine the reliability of each with respect to uranium exploration in the state.

Personnel consisted of G.R. Eakins, principal investigator, Br. R.B. Forbes with the University of Alaska Geophysical Institute, and C.L. Carver and B.K. Jones, graduate students at the University of Alaska who were geological assistants in the field and office. Jones performed much of the petrographic studies and Carver compiled the maps and geochemical data.

The field work was supported by a full-time helicopter during the 6 weeks in the field. Six separate base camps were occupied.

All the stream-sediment and bedrock samples were analyzed for th and $\mathrm{K}_{2} \mathrm{O}$ by the DGGS laboratory. Because of early problems with the analytical equipment, the DGGS contracted Resource Associates of Alaska (RAA) in Fairbanks to do the uranium analyses. Later in the program, however, the DGGS laboratory perfected the $U$ method by purchasing new equipment and reanalyzed many of the samples.

The water samples were analyzed by the University of California Los Alamos Scientific Laboratory (LASL) in Los Alamos, New Mexico in conjunction with their geochemical survey of several western states and Alaska under another ERDA contract. The Los Alamos work is being directed by Dr. R.R. Sharp, Jr. Also, splits of most of the streamsediment samples were analyzed by Los Alamos Laboratory. Therefore, two sets of $U$ values---one marked "RAA" and one "LASL" w-mare reported 
for most stream-sediment samples. Values reported by Los Alamos are generally higher than those reported by RAA.

Alaska is about one-fifth the area of the entire U.S. and includes a large variety of geologic settings that suggest many targets for the prospector. A cross section between Anchorage and Point Barrow, Alaska, has been shown to be generally similar to a cross section between San Francisco and Denver. However, mineral exploration in Alaska is faced with numerous difficulties and high costs. Because of the general lack of roads, the rugged terrain, and the remoteness of most of the state, most programs require full-time helicopter support. Many questions are unanswered regarding the behavior of uranium in arctic and subarctic climates and in metamorphic terrains. Research programs are needed to determine the most effective methods of exploring this vast region. Field work on one such program was completed for ERDA during 1976. This was an on-the-ground follow up of the aerial radiometric survey of the Copper River basin concluded by Texas Instruments during 1975. Although uranium exploration in Alaska is far behind that of the conterminous states, the tempo is increasing and ERDA and industry have conducted radiometric and geochemical programs during the past 3 years $(1974-76)$.

Uranium has been produced from only one locality in the state, the Ross-Adams mine on Prince of Wales Island in southeastern Alaska. About 120,000 tons of ore averaging approximately 1 percent $\mathrm{U}_{3} \mathrm{O}_{8}$ were produced from a small peralkaline granite stock of Late Triassic or Eariy Jurassic age (Lanphere and others, 1964). A number of radioactive prospects are present within a 70-square-mile area surrounding the stock (Mackevett, 1963; Stephens, 1971), but so far none of them has been found to be of commercial grade. Exploration in this area again is intense. Brief summaries of the history of uranium exploration in Alaska have been published (Eakins, 1969, 1975a, 1975b; Cobb, 1970).

No effort has been made in this report to define land status or ownerships. State land selections under the Alaska Statehood Act, selections by Native groups under the Alaska Native Claims Settlement Act, and Federal classifications are still pending. Present control and prior claims should be determined for each specific area of interest before any surface activities are undertaken. Because of the diversity of the geology among the several areas investigated, descriptions of the geology and results of the investigation of each area are included in separale sections.

\section{AREAS OF INVESTIGATION}

The two principa) areas (fig. 1) investigated by the DGGS party during the 1975 field season were: (1) west-central Alaska, including the Darby Mountains, Granite Mountain Clem Mountain area, Selawik Hills, and Zane Hills, and (2) the southwest part of the Copper River basin and parts of the Chitina River valley in the south-central part of the state.

These areas were chosen because the granitic rocks in west-central Alaska were known to be anomalously radioactive and to consist of alkaline rock types favorable for uranium associations, and because the Copper River basin contains nonmarine Tertiary sediments near granitic terrain which 


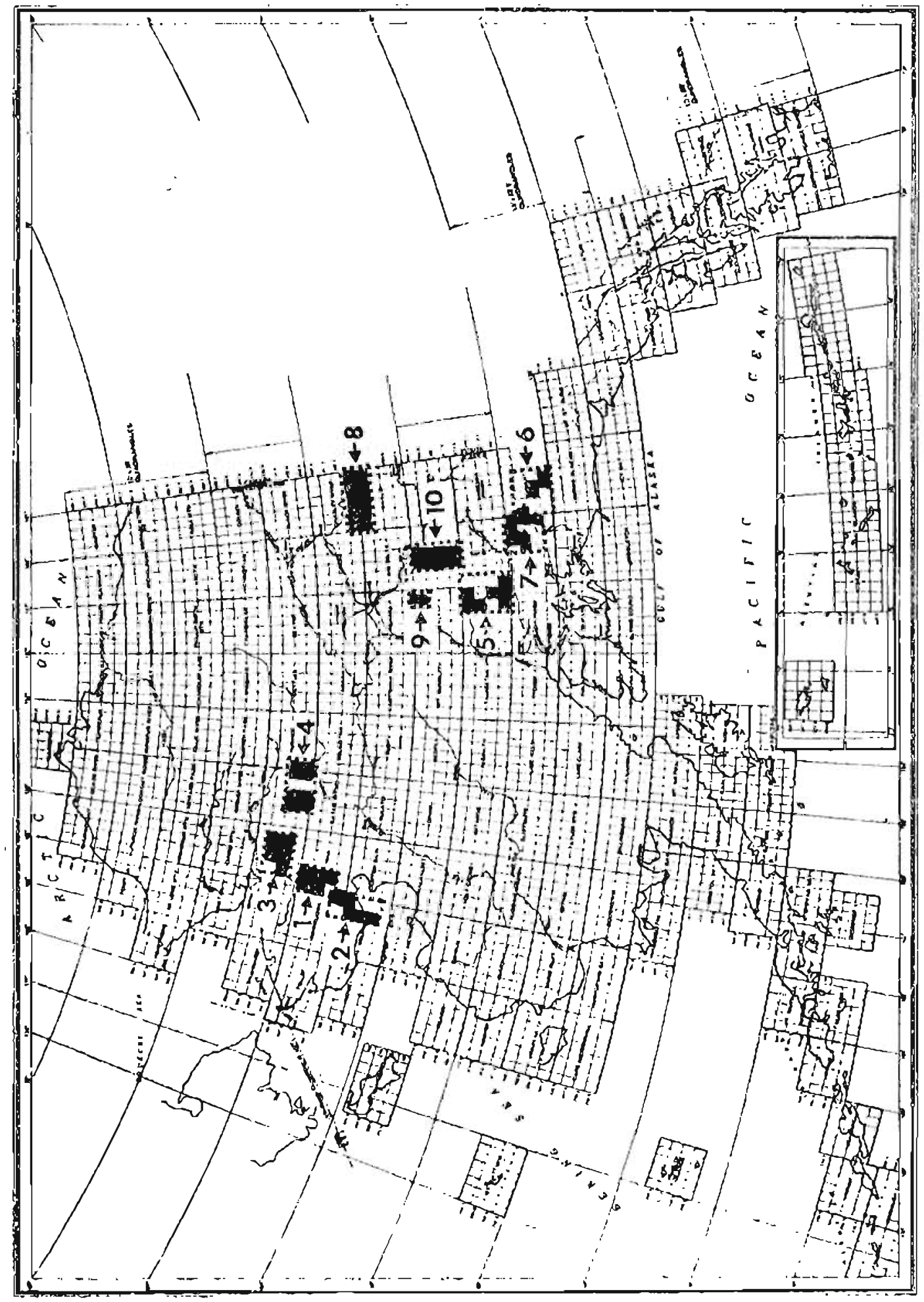

Figure 1. Index map of areas investigated for uranium by DGGS, 1975. Numbers refer to plates (in pocket). 
may be a setting similar to that of sandstone-type uranium districts in the western U.S. The Chitina River valley was included because it contains Mesozoic sandstones which have some of the characteristics of uranium-bearing sandstones and because it is near the Copper River basin. The investigation of one predominantly granitic area and one predominantly sedimentary area provided the opportunity of testing the sampling methods in both types of potential uranium provinces.

In addition to the two principal areas investigated, stream-sediment samples were collected by DGGS field parties in two other regions and analyzed for U, Th, and K. These areas are (1) the Healy D-I quadrangle and vicinity in the Healy coal district and (2) parts of the Eagle and Charley River quadrangles along the Yukon River and near the Canadian border.

The north flank of the Alaska Range contains nonmarine coal-bearing Tertiary deposits and has been investigated for ulanium by industry; it is still in an area of interest. The Kandik Basin has also been considered to have a potential for uranium in sediments of Precambrian, Paleozoic, and Tertiary ages.

Summaries of the general geology and previous investigations for uranium in these areas have been published previously (Eakins, 1975). Portions of that report are included in appendix $E$ for reference.

The general areas that were investigated are covered by the following 0.5. Ceological Survey 1:250,000 (4 miles-to-the-inch) topographic quadrangle sheets:

reneral area

West-central Alaska

Darby Mountains

Granite Mt. and Kunter Creek plutons Sellawik Hills

Zane Hills and Purcell Mits.

North flank Alaska Range

Healy district

East-central Alaska

Eagle-Charley River area

South-central Alaska

Southern Copper River basin

Chitina River valley nuadrangles

Solomon, Bendeleben

Candle, Selawik

Candle, Selawik

Shungnak, Hughes

Healy

Eagle, Charley River

Talkeetna Mountains, Gulkana, Valdez, Anchorage Valdez, McCarthy

Greater detail is provided by the USGS 1:63,360 (1 inch-to-the-mile) scale topographic sheets, which are available for all areas except the Shungnak, the western part of Selawik, and most of the Hughes quadrangles. All sample locations and field data were posted on a 1:63,360 scale. Maps of the are is not covered by 1:63,360 scale were obtained by enlarging the $1: 250,000$ topographic sheets. The sesults of the field work and sampling ty the DFGS in each of the eight above areas are discussed separately. 
Aerial photos of a 1:20,000 scale were obtained for the areas sampled in west-central Alaska and these were examined for structural features. Photos were not purchased for the Copper River region.

\section{FIELD METHOOS EMPLOYED}

\section{Stream-Sediment Sampling}

Silt-size sediments were collected from beneath the water of streams for $\mathrm{U}$, Th, and $\mathrm{K}_{2} \mathrm{O}$ analyses. Because of the large areas investigated during a relatively short time in the field, the sampling was of a broad reconnaissance nature. Most 5 treams were in mountainous areas and were very swift, but some sediments were also collected in streams in the lowlands bordering the highlands. These areas were tundra- or muskeg-covered and the water was sluggish or stagnant. These samples consisted mostly of carbonaceous material because mud was generally scarce.

In addition to sediment sampling at each location, an effort was made to collect a water sample for uranium analysis, make a radiometric measurement with a hand-carried scintillometer, and note the type of bedrock and stream float.

Sampling was done by two people working out of the helicopter. The number of stream-sediment samples collected during a working day was not great-- 20 to 30---because of the long distances to and from work areas, time lost for refueling, difficulty in finding landing places, relocating other members of the field party, and frequent difficulty in finding fine material suitable for a sediment sample. Whenever possible, approximately I cup of material was taken and placed in a cloth sample bag. The DGGS lab handled their analyses for $U$, Th, and $K_{2} O$. Sample splits were shipped to the Los Alamos Scientific Laboratory for analysis to accompany the water samples. Unfortunately, mislabeling of some of the splits of stream sediments and water shipped to Los Alamos caused many of the Los Alamos samples to be discarded.

\section{Water Sampling}

Water samples were taken at most of the sediment-sample locations. Plastic bottles were provided by Los Alamos, and the samples were shipped to their laboratory for analys is by the neutron activation method. Generally, two water samples were collected at each sample site: a $25-\mathrm{ml}$ vial and a 4-ounce vial. However, there were periods when the supply of vials became exhausted in the field and no water samples were taken.

The total number of water samples taken was 565 . The samples were collected by simply dipping the vial into running water, avoiding carbonaceous and particulate matter as much as possible. Prior to taking the sample the vial was rinsed in the water to be sampled and the sample taken by moving it up and down in a clear part of the stream to obtain a "cross section" of the flow. No special treatment was used except for rinsing the vials in dilute $\mathrm{HNO}_{3}$ before taking them to the field and adding 3 drops of concentrated $\mathrm{HNO}_{2}$ to the sample collected. Much more sophisticated techniques such as filtering and measuring the $\mathrm{kh}$ and $\mathrm{pH}$ of the waters are to be used in future water sampling by the Los Alamos Laboratory and others engaged in the NURE program throughout Alaska and the entire nation. 
In general, reports of water sampling for uranium in Alaska by industry have been rather discouraging. The geology and topography in Alaska are considerably different than those of the Canadian Shield province, where some success with the method has been achieved. Research is needed to construct models providing for the possible movement of uranium in the arctic and subarctic basins. A paper by the Russian V.P. Borovitskii (1975) touched on the effectiveness of geochemical exploration of swamps and bogs in permafrost regions, but little information on the geology and topography was included. Publications by the USGS (Williams, 1970; Pewe, 1975) provide data on the ground water in the permafrost regions of Alaska, and may be useful in any such studies.

\section{Bedrock Sampling}

The extensive exposures of Cretaceous alkaline plutons in the southeastern Seward Peninsula and west-central Alaska have been described by Miller (1970, 1972) and Miller and Bunker (1975). The rocks are unusually potassic and subsilicic. The abnormally high uranium and thorium contents of the Darby Mountains and the Rogatza plutonic belt suggest that they may be favorable areas for economic concentrations of uranium and thorium.

Forbes and Jones conducted the sampling and petrographic study of the granitic rocks. Their report on the mineralogy and $U$ and Th associations constitute a separate section of this report (part 11) and should be referred to for descriptions of the rocks in each area.

Systematic sampling of bedrock along selected traverses across the plucons was done to collect samples for $U, T h$, and $K_{2} O$ analysis and thin sectioning. The traverses were made along ridges where the exposures were best, and a sledge hammer was used to obtain samples as little weathered as possible. Forbes and Jones discuss the findings in the section on petrology, and tables and maps have been compiled to show the relations of the bedrock characteristics to the radiometric surveys and to the stream-sediment and water analyses. Of particular interest to a uranium polential study are determining the average $\mathrm{U}$, Th, and $\mathrm{K}_{2} \mathrm{O}$ contents of the various rock types, learning where the radioactive elements are contained within the rocks, and determining if leaching of the uranium has occurred.

For comparison of the $U$ and Th values obtained from analyses of rock and water samples collected for this study with general averages, it will help to keep the following figures in mind. Average uranium content of selected crustal rocks and major
rock types (W.I. Finch, 1967, p. 2)

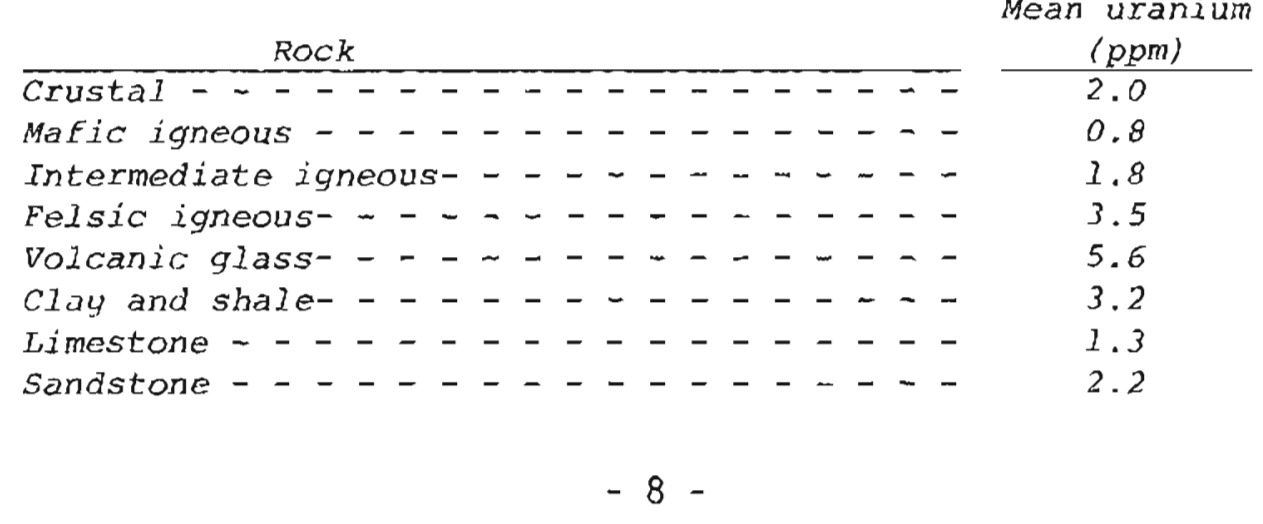


The value for felsic igneous rocks, around $4 \mathrm{ppm}$, is generally considered normal. Thorium concentrations of granitic rocks $\left(>70 \% \mathrm{SiO}_{2}\right)$ average 18 ppm (Wedephol, 1969, p. 92-8-2), and about $2 \mathrm{ppm}$ in plateau basalts. The $U:$ Th ratio in igneous rocks normally is about $1: 4$. The ratios of $U$ to Th and $\mathrm{K}_{2} \mathrm{O}$ in bedrock are believed to be significant and an aid in defining potential uranium-enriched districts. When the $U$ content approaches or exceeds the Th content, the ratio is anomalous and may indicate $U$ enrichment even though the actual level is relatively low. However, $U$ is more soluble than $T h$ in the zone of weathering and oxidation, causing the two elements to become separated and the ratio to change.

Weathering processes in the subarctic environment of west-central Alaska rapidly produce an accumulation of talus and rubble at outcrops because of the extreme temperature variations. Chemical weathering in the region, however, is retarded by permanently frozen ground and low rainfall, so that the rubble buildup is preserved. Because of the accumulation of debris, there are few good outcrops where the bedrock is exposed in place. Chemical weathering is more active in the Copper River basin-Chitina River valley region, where the climate is warmer and wetter.

Studies of the granitic rocks by Jones and Fotbes show that no general mineralogy guides can be applied to locate the most favorable uranium-rich localities in all areas, but each pluton has its own particular mineral assemblages associated with uranium and thorium.

\section{Ground Radiometric Survey}

Portable Mount Sopris single-channel scintillometers were carried by each member of the field party. Radioactivity measurements were made at most sample sites and are reported in the tables along with the analysis of each sample. All four instruments were calibrated to give the same responses under identical conditions. The meters indicated the radioactivity in counts per second (cps), which ranged from a low of 20 over tundra and some sediments to a maximum of 2,000 on a syenite dike in the Selawik Hills.

The measurements were generally made by placing the instruments directly on the ground or bedrock. The maximum reading obtained at each station was the one reported. The readings have been used to construct isorad maps of the areas examined in the southeastern part of the Seward Peninsula and west-central Alaska. The added bulk of this extra set of maps led to their reduction for inclusion within the text instead of printing them at the same scale as the sample location maps.

The Copper River basin radiometric surveys were not contoured, which, because of the consistently low values, would be of little use. The difference between the average radiometric measurements of the two regions is extreme. Whereas measurements of the bedrock in the Seward Peninsulawest-central region averaged 200-300 cps and occasionally reached 1200-2000 cps, those in the Copper River basin region seldom were as high as $100 \mathrm{cps}$ and were usually less than $60 \mathrm{cps}$.

Because stream-sediment samples and corresponding radiometric measurements were often obtained where no bedrock was exposed, readings were taken 
on the strean gravels. In some low areas neither bedrock nor float werc present and readings were taken on tundra. Although correlations beLween particular radiometric measurements and $U$ or th content of stream sediments and bedrock are often poor or nonexistent, they do exist for general areas. A discussion of the correlations between counts per second and $U$ and Th contents of the igneous rocks is included in the suction on igneous rocks by Forbes and Jones $(p .80)$.

\section{Aerial Radiometric Survey Data}

A highly sophisticated airborne radiometric and magnetic survey of the most of Copper River basin, the Seward Peninsula, and west-central Alaska was conducted by Texas Instruments, Inc. (1975) for ERDA during the summer of 1975. The aerial survey intentionally covered areas being investigated on the ground by the DGGS. A high-sensitivity multichamml gamma-ray system was flown at a $6.25-\mathrm{mile}(10-\mathrm{km})$ spacing al an average a)titude of 400 feet ahove the ground. The system scanned a 800 -footwide strip ot this altitude.

The anomalies presented on the Texas Instruments anomaly maps worc determined statistically, and represent values in standard deviations above or below the mean value for each particular rock unit. This methol depends on the accuracy of geologic mapping and rock classifications, which is still uncertain in some area. "Preferred" anomalies indicate $U$ enrichment over Th or $K$ (or both). The listings of the raw data, point by poinl, are needed for detailed study and interpretations, but these frive not been nade available in the Texas Instruments open-filed report $(6,00-1653)$.

The flight lines and the anomalies as determined by Texas Instruments have been transferred to the sample location-geology maps accompanying this report in an effort to correlate as many of the available data as possible. For more information on these anomalies the reader is referred to fiJo-1653.

The Texas instrumenls report on the survey stated:

The principal problem encountered wes low counting iates, ospccially in the basin areas caused by watcr-shiclding by the satuxated surfacr materials. This may have effectively prevented detection of any uraniforous provinces which might be prosent in rhose areas.

Becausc of the nature of the intrusive rocks and relatively thin suficial cover, anonalies are much more numerous in the Scward Peninsula.... west-central Alaska region than in the Copper River basin region.

Follow-up ground studies to determine the cause and significance of the aerial radiometric anomalies encountered in Alaska are being initiated by ERDA. Field parties will make detailed examinations of the anomalic, with hand-carried multichannel spectroneters and by detailed mapping. The first follow-up project, conducted in the Copper River basin rluring the summer of 1976, will be an aid in interpreting the radionetric data .nd determining if leaching and migration of uranlum has occurred, and will also be a general aid in uranium exploration of lowlands in Alaska. similar follow-up studics are planned for other areas within the stata. 


\section{SUMMARY OF RESULTS}

Summaries of the results of the program are discussed by principal areas. Plates showing sample locations and the aerial radiometric anomalies discussed accompany the report. Sample analyses and statistics appear in appendixes $A, B$, and $C$. Correlation matrices are listed in appendixD. The general geologic setting of each area is summarized in appendix E.

\section{Granite Mountain Area}

The fleld party was based at the White Alice communications facility located on the top of Granite Mountain in the western part of the Candle quadrangle $(p 1.1)$. Field wark was possible only 5 of the 9 days at this location because of the weather. The areas sampled include the Granite Mountain pluton with its satellitic body on the northeast side, the nuartz Creek pluton, and the Hunter Creek pluton. General locations of the plutons of west-central Alaska are shown on figure 2.

\section{Stream-Sediment Sampling}

A total of 146 stream sediment samples were collected in this area. Results of the analyses are:

\begin{tabular}{|c|c|c|c|c|}
\hline & Range & Mean & Threshold & Anomal ies \\
\hline Uppm (RAA) & $n-8 \overline{8}$ & 6.4774 & 23.5104 & 3 \\
\hline Uppm (LASL) & $0.14-91$ & 13.8012 & 44.061 & 7 \\
\hline Th ppm & $0-79$ & 20.5366 & 51.404 & 7 \\
\hline $\mathrm{K}, 0 \%$ & $0.64-1.67$ & 1.9384 & 3.432 & 5 \\
\hline
\end{tabular}

The highest U value reported, 88 ppm (sample B137), was from near the head of Doc Creek, a small north tributary to Sweepstakes Creek on the south flank of Granite Mountain. The Th value for this sample was $25.8 \mathrm{ppm}$, which results in a U:Th ratio of 3.41:1. However, the radiometric reading at this sample location was only 20 cps, and the bedrock is basalt or andesite. Another sediment sample (B/40) collected about 1 mile downstream from $B 137$ yielded only $2.2 \mathrm{ppm} U$ and $5.59 \mathrm{ppm}$ Th. Additional sampling is needed near the head of Doc Creek to determine the source of the uranium in sample 8137 .

Six other unusually high values range between 18.0 and $28.0 \mathrm{ppm}$. Three of these---361, B62, and B71--were collected in the headwaters of the Peace River on the northeast flank of Granite Mountain. Panned concentrates front this area have previously been reported to contain anomalously high radioactivity, uramium-bearing minerals, and a variety of sulfides (Gault and others, 1953; Elliott and Miller, 1969; and Miller and Elliott, 1969). Bedrock exposures are almost entirely lacking, but four small pits dug in 1952 along the north fork of the northernnost part of the Peace River revealed weathered granitic rock. A. small stock satellitic to Granite and consisting of monzonite, syenite, and nepheline syenite has been mapped in this area by Miller and Elliott (1969, figs. 1, 7). The area is covered by tundra and several feet of soil, but is well drained and completely free of brush so that further exploration would be relatively easy. 


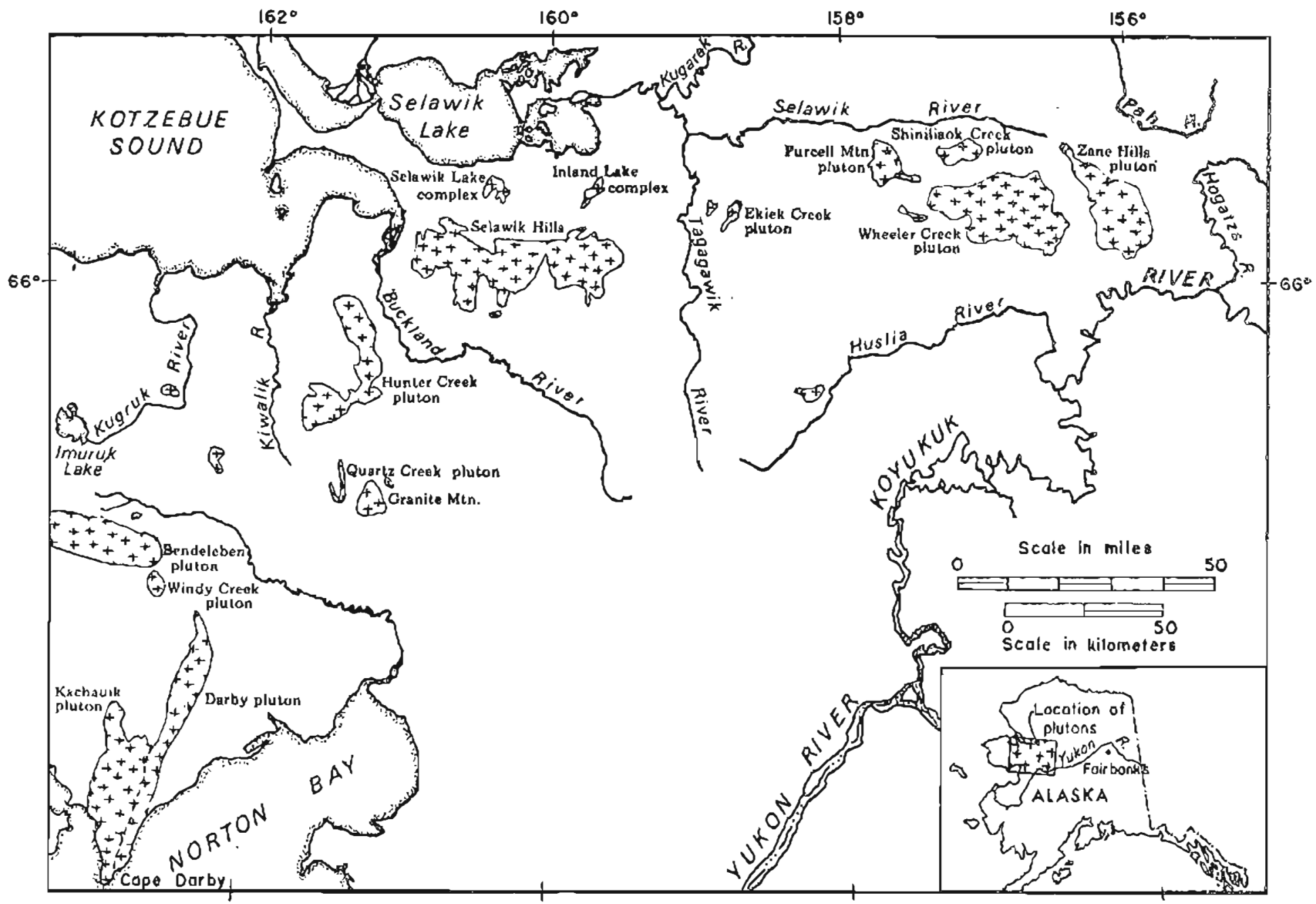

Figure 2. Plutons of west-central Alaska.

A sediment sample ( $B 30)$ from a stream 4 miles south of Clem Mountain yielded $18.8 \mathrm{ppm} \mathrm{U;} \mathrm{sample} \mathrm{B5I,} \mathrm{from} \mathrm{near} \mathrm{the} \mathrm{south} \mathrm{end} \mathrm{of} \mathrm{the} \mathrm{Hunter} \mathrm{Creek}$ pluton, produced $20.3 \mathrm{ppm} \mathrm{U}$. The latter sample was collected near or on a fault mapped by Patton (1967).

Numerous other sediment samples from the Granite Mountain-Hunter Creek pluton area, i.e., Anzac, Boulder, Connally Creeks, and others, contained above-average amounts of uranium $(10-15 \mathrm{ppm})$. Most were from the Granite Mountain pluton. The west side of the Hunter Creek pluton is anomalously high in thorium---up to $78 \mathrm{ppm}$, and uranium up to $12.5 \mathrm{ppm}$. The highest Th and $\mathrm{K}_{2} \mathrm{O}$ values usually were from the samples having the higher uranium values. 
Water Sampling

A total of 134 water samples were taken in the Granite MountainHunter Creek pluton area:

$\mathrm{H}_{2} \mathrm{O}(p p b) \quad \frac{\text { Range }}{0-2.15} \quad \frac{\text { Mean }}{0.7508} \quad \frac{\text { Threshold }}{1.5936} \quad \frac{\text { Anomalies }}{8}$

Four of the water anomalies were in samples B25, B27, B31, and B44, collected over granitic rocks of the Hunter Creek pluton, between flight Iines 62 and 63 . Three more (B62, 865, and B72) were from the headwaters of Peace River on the northeast flank of Granite Mountain, and one (B/18) was from Anzac Creek.

Bedrock Sampling

A total of 153 rock samples were collected in the Granite MountainHunter creek pluton area for analysis and thin sectioning. The highest uranium and thorium contents by chemical analysis and radiometric measurements were obtalned from the syenites or nephline syenites of Granite Mountain. The ranges in the chemical analyses of the rock samples were:

\begin{tabular}{|c|c|c|c|c|}
\hline $\mathrm{U} * \mathrm{ppm}$ & $\frac{\text { Range }}{0-258}$ & $\frac{\text { Mean }}{3.6898}$ & $\frac{\text { Threshold }}{10.1592}$ & $\frac{\text { Anomalies }}{5}$ \\
\hline Th ppm & $0-290$ & 25.5889 & 67.8015 & 10 \\
\hline $\mathrm{K}_{2} \mathrm{O} \div \%$ & $0.10-9.15$ & 4.0735 & 8.9503 & 3 \\
\hline
\end{tabular}

*BI49R and $8151 R$ not used in calculating threshold because of extremely high values.

The highest single $U$ and Th values as shown above are highly anomalous, and the second highest values were $29 \mathrm{ppm} U$ and $93 \mathrm{ppm}$ Th. The sample containing $258 \mathrm{ppm} U$ (BI50R) was from nepheline syenite dikes on the southwest flank of Granite Mountain, just a few hundred yards west of the northern end of the landing field.

Most of the bedrock in the area has been reduced to rubble, but boulders of the radioactive nephel ine syenite are up to 3 feet long and 1 foot thick. The syenite occurs as banded dikes cutting a highly fractured and veined diorite(?) porphyry. Scattered grains in the nepheline syenite fluoresce a bright orange. The Th content was $32.8 \mathrm{ppm}$.

The highest thorium value $(289 \mathrm{ppm})$ was from a sample (B152R) of a radioactive boulder found near the head of Granite creek. The $U$ content was only $10.9 \mathrm{ppm}$, so the highest Th and highest $U$ were not from the same sample but were from the same general locality.

Two bedrock samples collected at Granite Mountain which produced very high radiometric readings and anomalous $U$ or Th values were submitted to the DGGS laboratory for mineralogical analyses. One sample (8149R) was from the nephel ine syenite dikes which contains $258 \mathrm{ppm} U$. It was found a few hundred yards west of the northern end of the runway on Granite Mountain. The second sample (B151R) was from a boulder near the head of Granite Creek on the southwest flank of Granite Mountain. The analyses by size fractions and their radloactivity are given in tables 1 and 2 . 
Table 1. Mineral composition, SElR

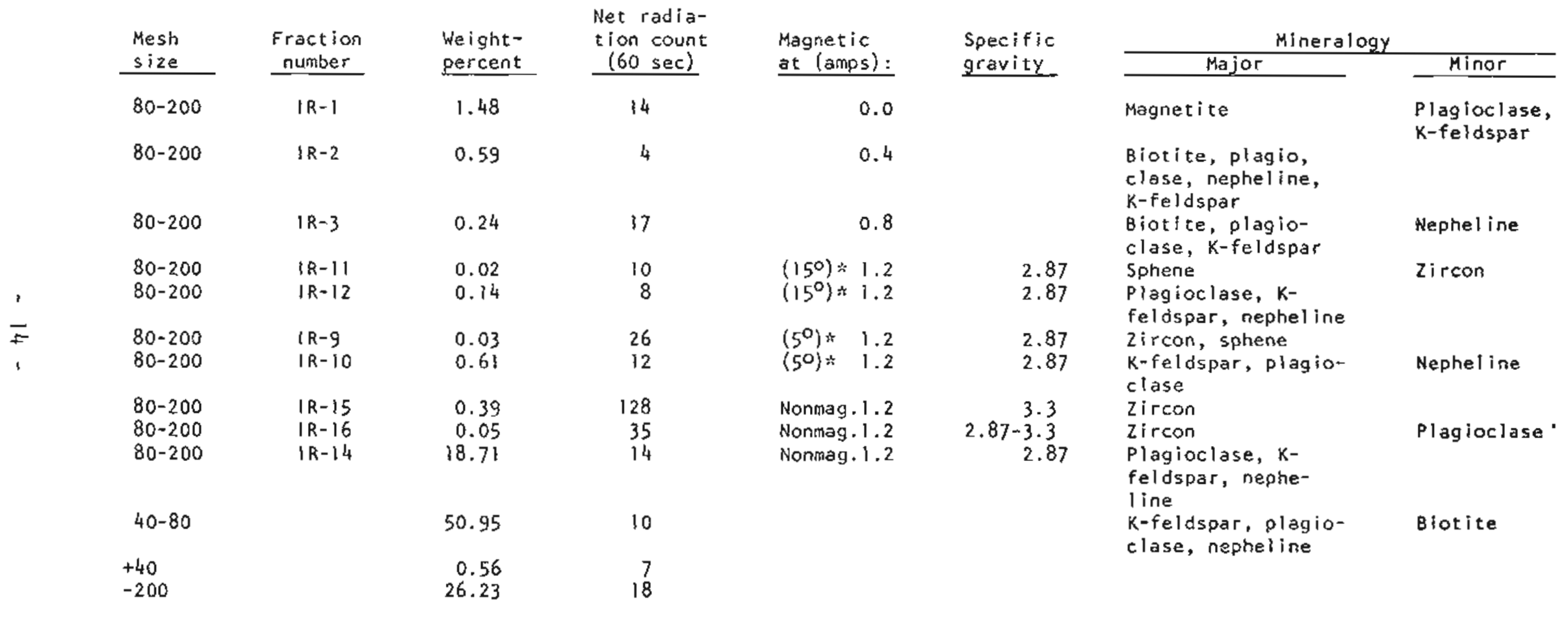

Fide slope at Franz magnetic separator. 

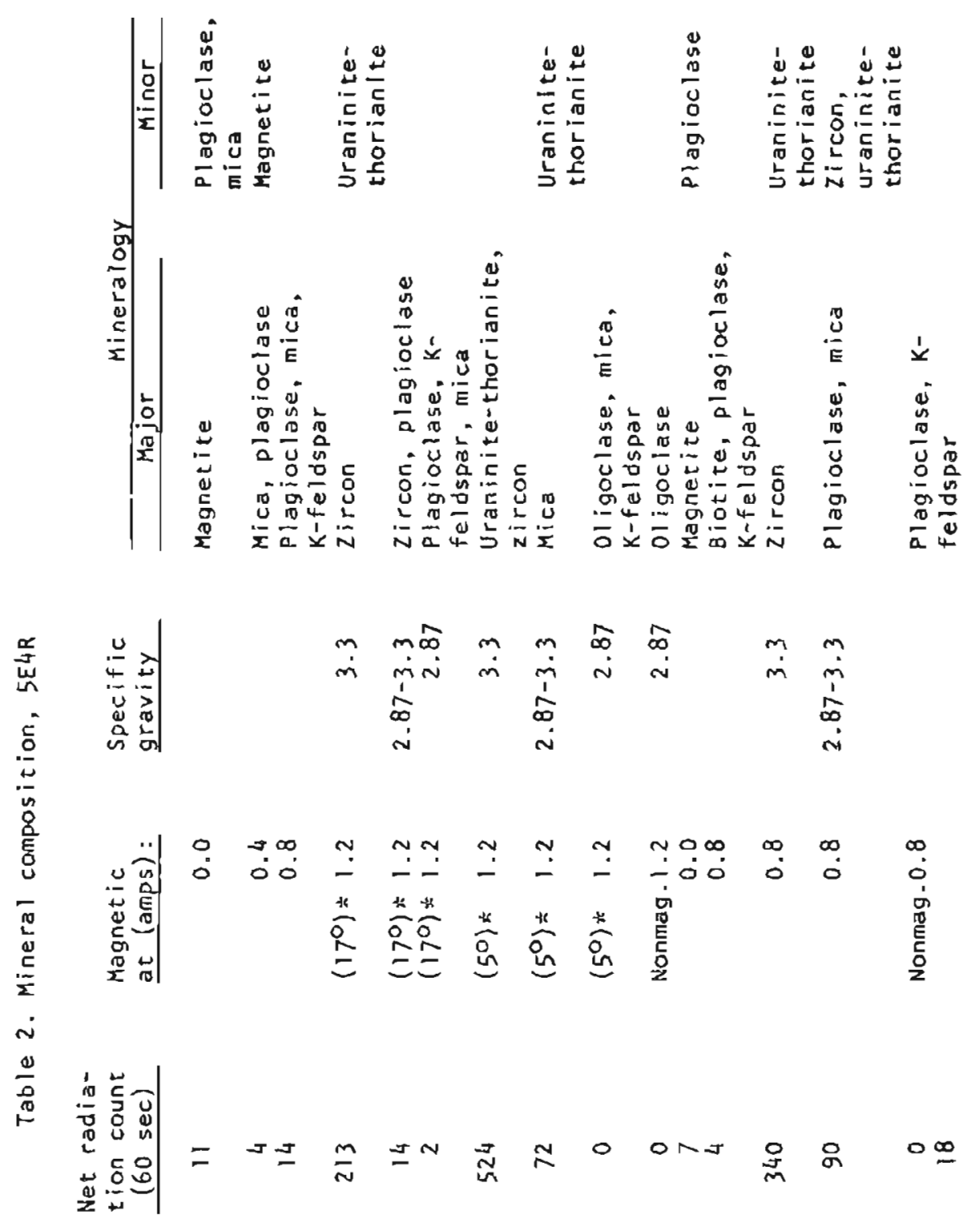

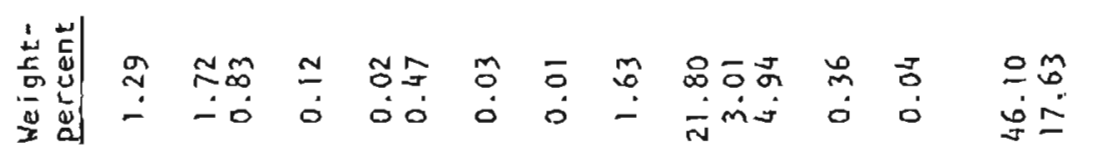

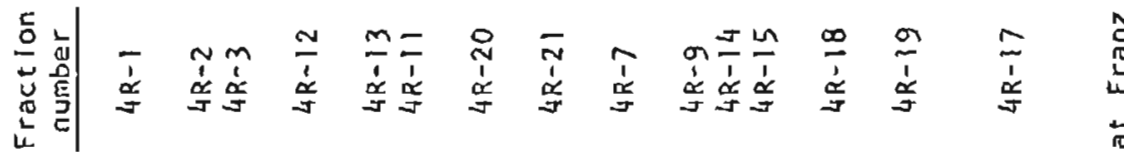

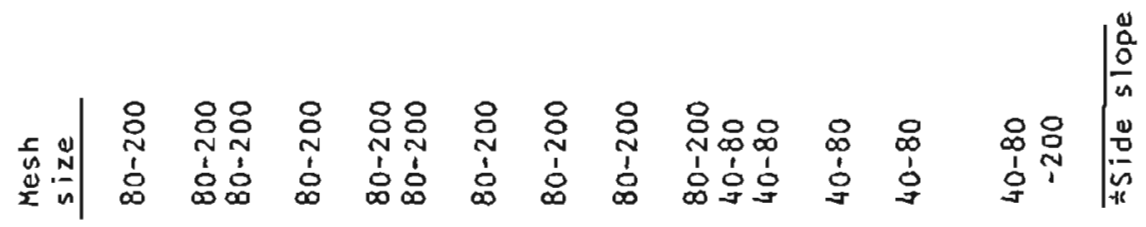


The flight lines and radiometric anomalies from the Texas Instruments survey are indicated on the accompanying sample location maps. Flightline 65 crosses 2 miles north of Granite Mountain summit in an east-west direction and, fortunately, crosses the headwaters of Peace River. A "suspect" anomaly is indicated at the Peace River locality, where streamsediment samples and ground radiometric surveys also produced uranium and thorium anomalies. Another aerial anomaly along flight-line 65 was found 6 miles west of the Peace River anomaly, over a nepheline syenite zone on the western margin of the Granite Mountain pluton.

Two suspect anomalies appear on flight-line 62 in the Hunter Creek area of the Hunter Creek pluton, where anomalous Th values were obtained from stream-sediment samples. One suspect anomaly is along flight-line 60,10 miles southwest of Buckland.

Aerial radiometric anomalies east of the area investigated by the OGGS suggest that the cretaceous sediments should be investigated for their uranium potential. These occur near the center of the Candle quadrangle and contain some volcanic debris and plant remains (Patton, 1967).

Ground Radiometric Survey

Scintillometer readings in the Granite Mountain-Hunter Creek pluton area ranged from a low of $17 \mathrm{cps}$ over basalt to a maximum of $1,200 \mathrm{cps}$ on nepheline syenite dikes on the southwest side of Granite Mountain (figs. $3,4,5)$. Counts measured over lavas were generally less than 50 cps. Counts over monzonite and quartz monzonite varied between 100 and 250

cps. Syenites usually produced between 200 and 300 cps.

An area of about 1 square mile at the head of Peace River is particularly interesting because exceptionally high readings (up to 370 cps) were obtained over tundra and soil in the stream banks. Stream gravels also produced up to $300 \mathrm{cps}$. These levels of radioactivity are unusually high even for exposed granitic bedrock, and generally a thin cover soil and tundra will completely mask radiation. But in this area there are no bedrock exposures except in a few test pits along the stream channel, and soil and tundra are several feet thick. Radioactive material has been incorporated into the overburden. Thorium was found to be above average in the sediment samples (up to $35.3 \mathrm{ppm}$ ) and the uranium was as high as 28 ppm.

"Contouring" ground radiometric measurements produced a broad high over the central part of Granite Mountain. One pronounced anomaly appears on the west side of the landing strip. where the radioactive nepheline syenite dikes were found, and one centers in the headwaters of Peace River.

There was a general decrease in radiometric measurements northward from Granite Mountain along the Hunter Creek pluton, but another "high" appears at the head of Spruce Creek, a tributary to Hunter Creek. 


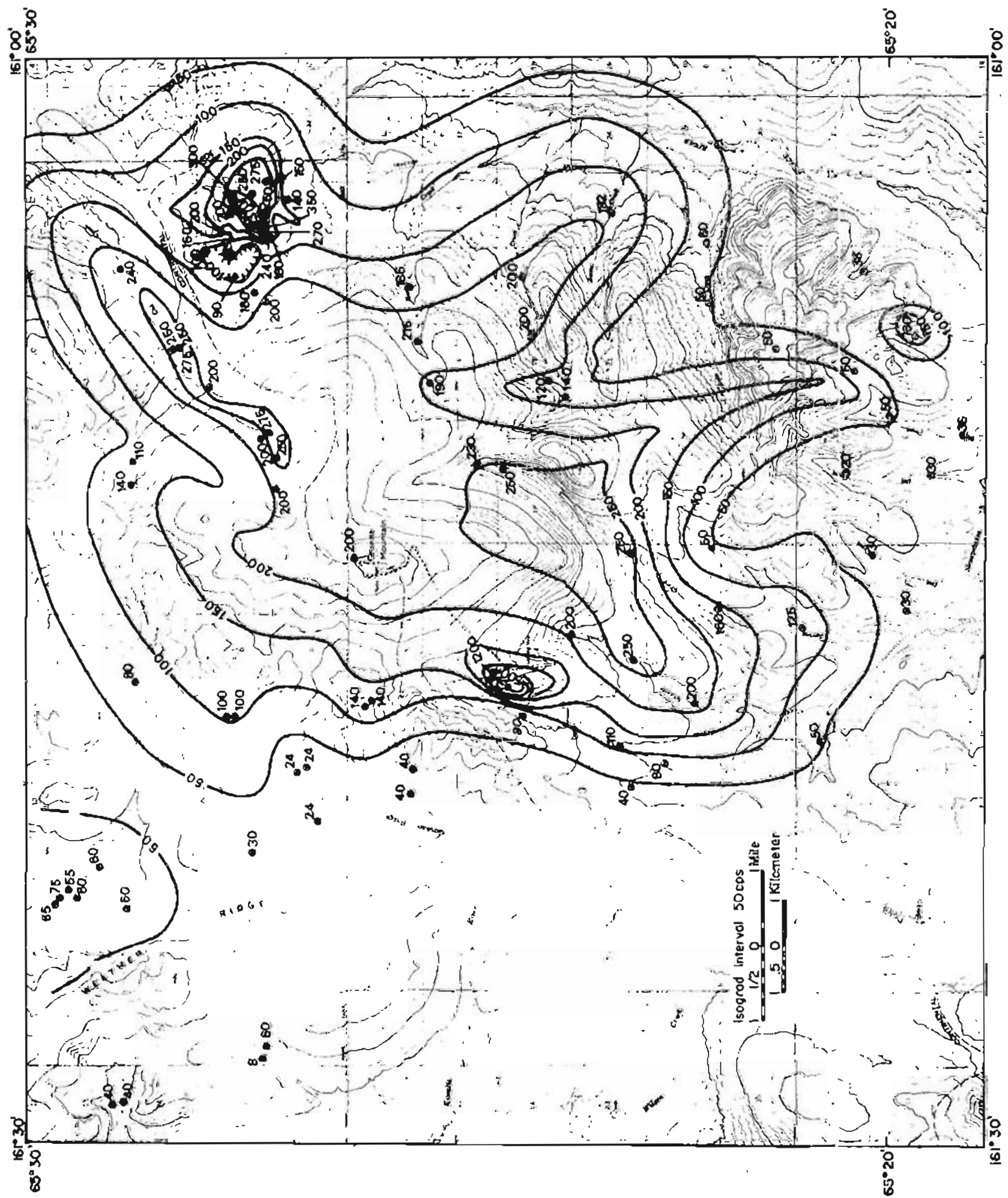

Figure 3. Ground radlometric survey, Granite Mountain area, Candle B-5 quadrangle; by G.R. Eakins and C.L. Carver, 1976. 


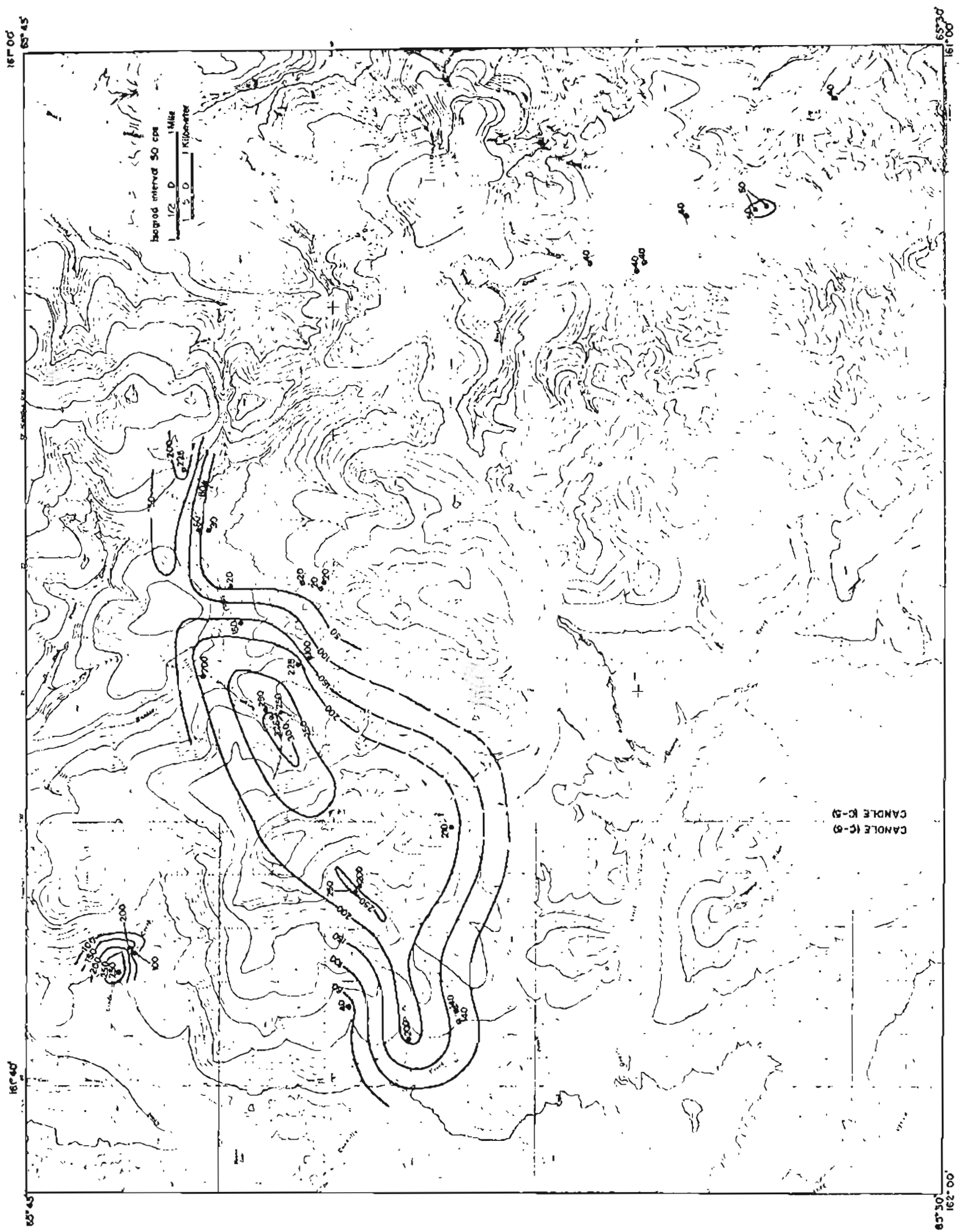

Figure 4. Ground radiometric survey, part of Hunter creek pluton, Candle $\mathrm{C}-5$ and C-6 quadrangles; by G.R. Eakins and C.L. Carver, 1976. 


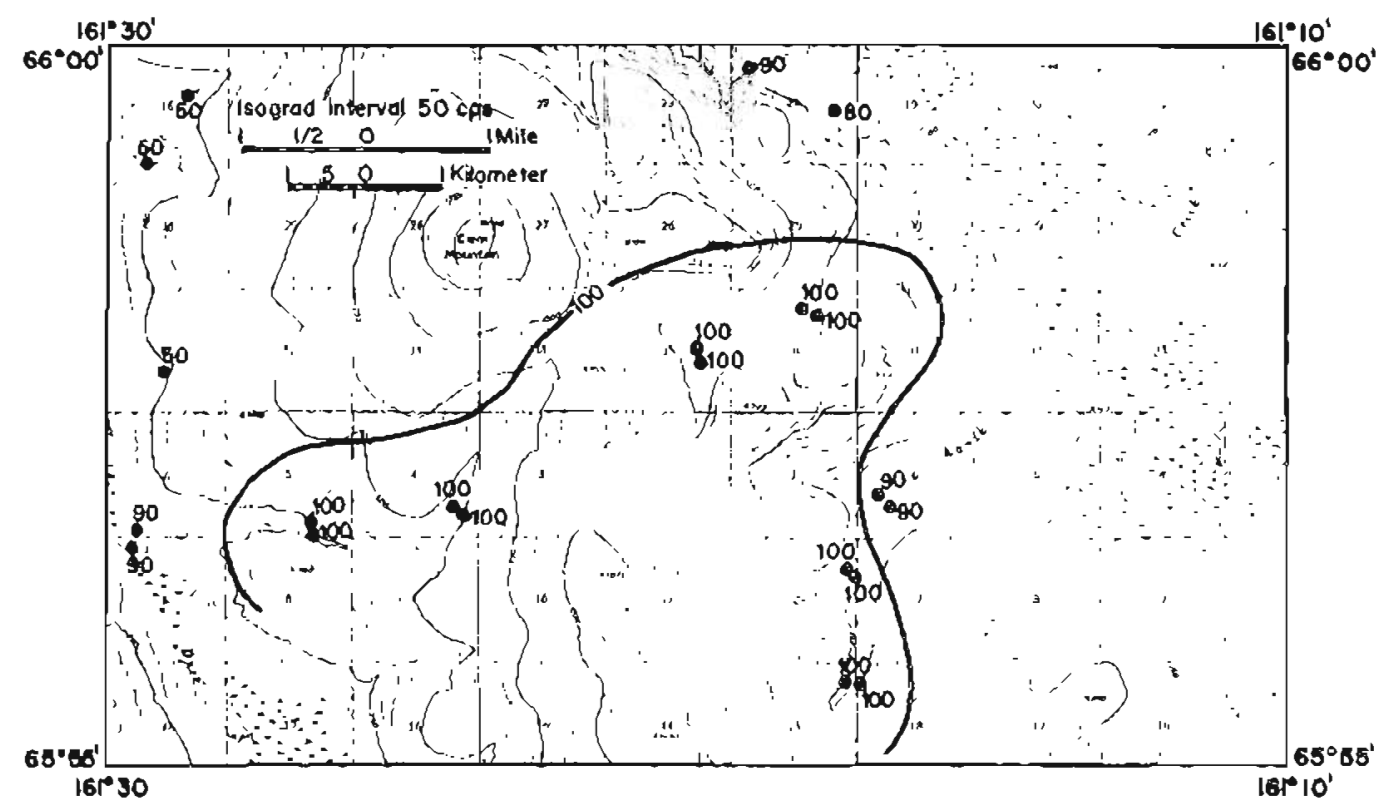

Figure 5. Ground radiometric survey, northern part of Hunter Creek pluton; Candle D-5 quadrangle, by G.R. Eakins and C.L. Carver, 1976.

Suggestions for Exploration

The headwaters of Peace River have been known for many years to contain radioactive material, and it still appears to the writer to be one of the most intriguing localities for exploration. Anomalous $U$ and Th have been found in stream gravel concentrates and stream-sediment. samples, and both ground and aerial radiometric surveys have shown the area to be anomalous. Mineralization is believed to be associated with a small monzonite-syenite stock. The area would be relatively easy to explore by drilling. Uraninite or thorianite (or both) were identified in a sample of a granitic boulder on the south side of Granite Mountain.

More detailed studies and sampling are needed to localize uranium prospects in the Granite Mountain-Hunter Creek pluton area. Radiometric and geochemical anomalies are rather widespread around the margins of Granite Mountain and along the western side of the Hunter Creek pluton. Forbes and Jones suggest that rocks of the Granite Mountain pluton most favorable for uranium associations are those containing abundant nepheline and garnet but little plagioclase and amphibole.

The Cretaceous sediments near the center of the Candle quadrangle probably warrant investigation. Compositions of the sediments and aerial radiometric anomalies indicate a favorable area for exploration.

The Darby Mountains

The field party was based at Moses Point, an abandoned FAA station on Norton 8ay, while conducting sampling of the Darby Mountains area (pl. 2). The area is in the eastern Solomon quadrangle and the southeastern corner of the Bendeleben quadrangle. Plutonic rocks and streams were sampled from Cape Darby, on the southern end of the Darby Peninsula, northward 60 miles to Death Valley on the east side of the range. On the western side, the northernmost point reached was the Omilak mine on Omilak Mountain. The geologic setting is described in appendix E. 
Stream-Sediment Sampling

A total of 163 stream-sediment samples were collected in the area. Results of the analyses are:

\begin{tabular}{|c|c|c|c|c|}
\hline & Range & Mean & Threshold & Anoma\} i es \\
\hline Uppm (RAA) & $0-81$ & 14.8669 & 49.2515 & 15 \\
\hline Uppm (LASL) & $6.8-111$ & 20.7463 & 60.1325 & 7 \\
\hline Th ppm & $4.4-150$ & 33.7210 & 87.3388 & 8 \\
\hline $\mathrm{K}_{2} 0 \%$ & $0.76-5.5$ & 2.6492 & 4.3178 & 7 \\
\hline
\end{tabular}

Agreement between the $U$ analyses reported by RAA and LASL is reasonably good for the sediment samples collected in the Darby Mountains area. Average values for both $U$ and $T h$ are high in the stream sediments. A group of sediment samples (J20-J65) from an area west of Vulcan and Clear Creeks in the Solomon D-1 quadrangle are almost all unusually high, up to $81 \mathrm{ppm}$ $U$ and $99 \mathrm{ppm}$ Th.

Ground radiometric readings were consistently above average (200 $600 \mathrm{cps}$ ) and the aerial radiometric survey also revealed an anomaly in the D-l quadrangle (flight-line 71 ).

The Dry Canyon stock on the west flank of the Darby Mountalns produced a highly anomalous stream-sediment sample $(77 \mathrm{ppm})$ and the highest Th value produced in the Darby Mountain area (149.5 ppm).

Bedrock samples collected on the ridges in the same areas as the stream-sediment samples were much lower in uranium content than were the sediments. It appears that uranium has been weathered from the granitic rocks and concentrated in the fines in the streams (especially samples J20-J65), whereas thorium has not migrated significantly. Another possibility for the great disparity in uranium values to consider is that the $U$ extraction from the bedrock by laboratory methods was much less than from the stream sediments. It may be that both the natural and laboratory processes are factors. Since the Los Alamos Laboratory did not do $U$ analyses for bedrock samples, there are no delayed-neutronactivation analyses for comparison.

Water Sampling

A total of 126 water samples was callected in the Darby Mountains area, generally from stream-sediment sites. Results of the water analyses for $U$ are:

$\mathrm{H}_{2} \mathrm{O}$ ppb $\frac{\text { Range }}{0.3-2.3} \quad \frac{\text { Mean }}{0.9352} \quad \frac{\text { Threshold }}{1.984} \quad \frac{\text { Anomalies }}{7}$

Overall there is some correlation of high $U$ values in the waters to those in corresponding sediment samples. Although the water values are mostly in a low range, the higher ones may relate to anomalous amounts of $U$ in the bedrocks. Four of the anomalous values are 1 to 3 miles south of the aerial radiometric anomaly on flight-line 71 . 
Bedrock Sampling

A total of 117 bedrock samples were collected in the Darby Mountains area, mostly along an east-west traverse near Vulcan Creek. Bedrock analyses produced the following:

\begin{tabular}{|c|c|c|c|c|}
\hline & Range & Mean & Threshold & Anomalies \\
\hline J ppm & 0.20 .3 & 5.1074 & 12.496 & 5 \\
\hline Th ppm & $0-85.6$ & 32.6709 & 74.9435 & 4 \\
\hline $\mathrm{K}_{2} 0 \%$ & $0.02-11.18$ & 5.4809 & 10.7727 & 8 \\
\hline
\end{tabular}

The Darby Mountains are enriched in silica. Although the $U$ content of some rock samples is unusually high for granitic rocks, the values are generally considerably lower than those of the stream sediments. Thorium, on the other hand, is rather consistently high. Above-average $U$ was obtained from samples in the series $31 R-J 72 R$, from the northeast side of the Darby Mountains in the Solomon $D-1$ quadrangle. The area is underlain by quartz monzonite of the Darby oluton and is drained largely by Clear and Vulcan Creeks.

The corresponding Th content of this series of samples is anomalously high and in greater proportions than that normally found in igneous rocks; this results in a relatively low U:Th ratio, suggesting a Th province.

Two small outcrop areas on the west side of the Darby Mountains are exposures of the Dry Canyon Creek stock. It is predominantly a hornblendebiotite-nepheline syenite consistently high in Th (to $76.0 \mathrm{ppm}$ ) and slightly anomalous in $U$ (up to $20.3 \mathrm{ppm}$ ). Forbes and Jones found that the samples containing abundant accessary minerals and little nepheline were the best hosts for uranium.

At the time of this writing, the U.S. Department of Interior issued a news release on a uranium-thorium-rare earth find by the USGS in the Kachauik pluton of the Darby Mountains. A 13-page USGS open-file report (Miller, Elliott, Finch, and Brooks, 1976) has been released to the public (Sept. 27, 1976). The DGGS field party did not reach the particular sites of the discoveries, but did conduct sampling within 4 miles of them.

The information in the USGS report is significant to this study and a considerable portion is reproduced here with location maps (figs. 6 and 7).

Uranium-, thorium, and rare-earth bearing rocks were found by a U.S. Geological Survey field party 15 miles northeast of Golovin, Alaska, in the southeastern Seward reninsula (fig. 6) in June 1976. The mineralized areas occur in syenite and appear to be concentrated. along the margins of alkaline dikes, with allanite tentatively identified as the principal mineral containing the uranium-, thorium-, and rare-earths. Samples contain as much as 0.15 percent $\mathrm{U}_{3} \mathrm{O}_{8}$ and 1.05 percent $\mathrm{ThO}_{2}$, and over 2 percent rare-earth elements. These mineralized rocks are closely associated with alkaline dikes which are part of a dike swarm that crops out over at least $250 \mathrm{~km}^{2}$ (100 $\left.\mathrm{mi}^{2}\right)$. This large dike swarm is thus of considerable economic interest. 


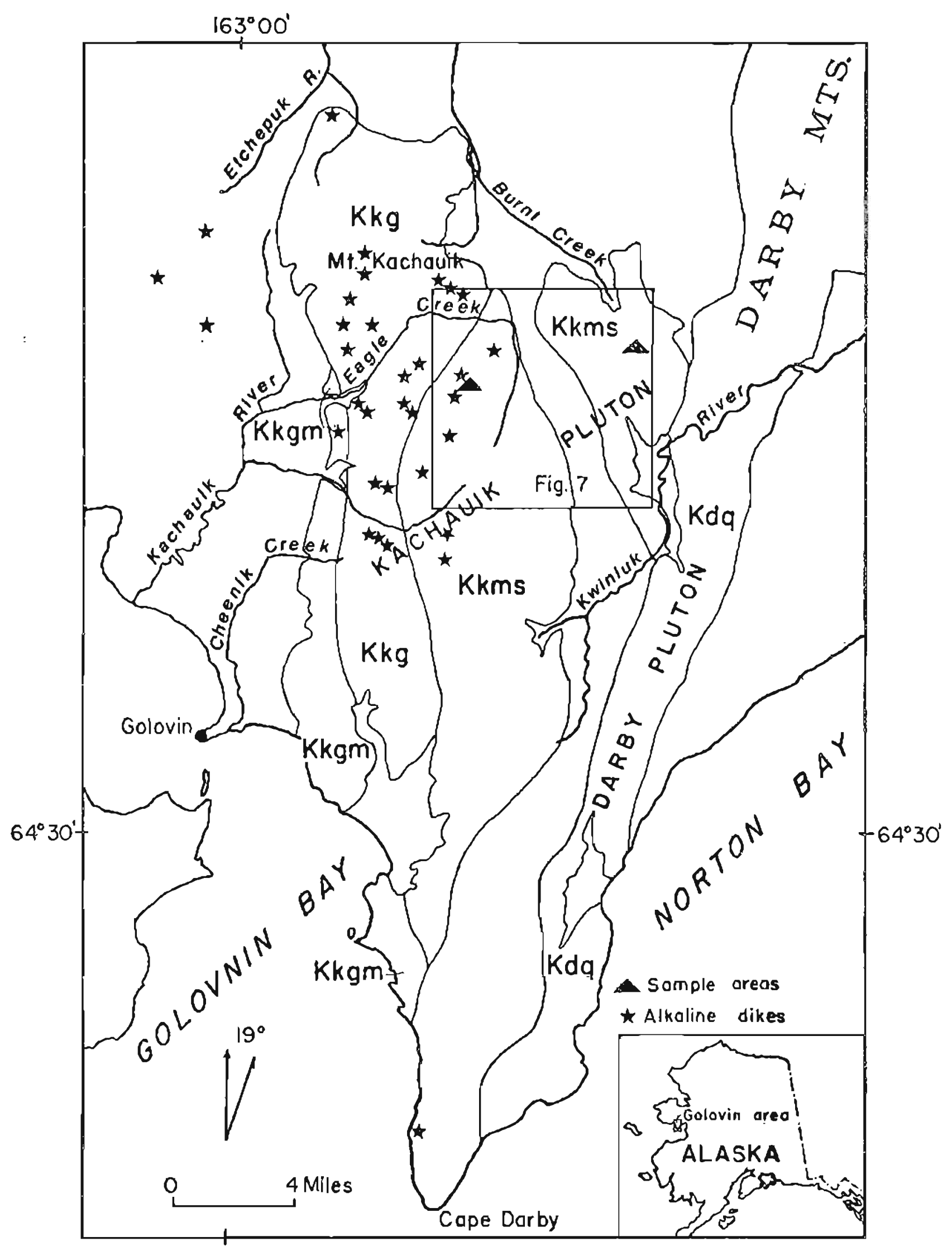

Figure 6. Generalized geology of the Darby Mountains area. Sources: Miller, Elliott, Finch, and Brooks, 1976. 
The principal mineralized area occurs on top of a small flat knoll shown as hill 2109 in the SW 1/4 of SeC. 18, T. 9 S., R. 20 W. on the Solomon $C-2$ 1:63,360 quadrangle map, (fig. 7) located on the crest of a north-south ridge bounded on three sides by Eagle creek (fig. 7). The mineralized rock consists of medium- to coarse-grained syenite characterized by large (a.s much as $1.25 \mathrm{~cm}$ or 0.5 in) brownish-black allanite crystals constituting 20 to 40 percent of the rock. Because of extensive frost-heaving, none of the allanite-rich rock can be found actualiy in place; however, abundant pieces of float of this material as much as $30 \mathrm{~cm}$ ( $12 \mathrm{in}$ ) across occur scattered over a zone $9-14 \mathrm{~m}$ (30-45 ft) wide and extending some $60 \mathrm{~m}$ (200 ft) across the top of the knoll. This zone lies near the east margin of a northelast-striking pulaskite dike which extends for some $900 \mathrm{~m}(3,000 \mathrm{ft})$ along the ridge crest. float of allanite-bearing syenite was found in several other places over a distance of $450 \mathrm{~m}(1,500 \mathrm{ft})$ along the eastern margin of the dike south of hill 2109 suggesting the mineralized zone may have considerable stxike length. The dike is off-set as much as $15 \mathrm{~m}$ by faulting in at least two places.

The actual width of the mineralized zone is difficult to determine owing to the lack of true outcrop. The larger talus blocks of nonmineralized monzonite and sycnite tend to mask the smaller blocks of allanite-bearing suenite on the slopes and on the ridge crest away from the flat-topped knoll at hill 2.109.

The allanite-bearing syenite is strongly radiaactive with a total count of up to 8,000 cps being recorded for pieces of float up to 12 inches across. This is 20 to 2.5 times background for the syenite and monzonite of the Kachauik pluton. In addition to allanite, the mineralized rock contains K-feldspar, plagioclase, and nepheline with minor hornblende and biotite. "zircon, apatite, and sphene are present as accessories. Nepheline has been previously noted at a few localities in the Kachauik pluton, but it does not appear to be widespread. Its occurrence next to a nepheline-bearing dike suggest that it may have been metasomatically introduced, perhaps along with the allanite.

Other pulaskite dikes occur on the west side of hjll 2109 and strongly radioactive allanite-bearing syenite float was found along the margin of at least one of these dikes (sample 1, table 3).

Samples of the strongly radioactive allanite-bearing syenite and of the pulaskite dike were analyzed for uranium and thorium. These samples were selected as being typical of the most radioactive material found and were taken from float blocks as much as $30 \mathrm{~cm}$ (12 in) across. The uranium content of the radioactive allanite-bearing syenite averages $1.325 \mathrm{ppm}\left(0.156\right.$ percent $\left.U_{3} \mathrm{O}_{8}\right)$ by neutron activation analysis and thorium averages $7,990 \mathrm{ppm}$ $\left(0.91\right.$ percent $\left.\mathrm{ThO}_{2}\right)$. A sample of the pulaskite (sample 2) dike yielded $34 \mathrm{ppm} U\left(0.004\right.$ percent $\left.U_{3} \mathrm{O}_{8}\right)$ and $96 \mathrm{ppm}$ th $(0.011$ percent $\mathrm{ThO}_{2}$ ), which is about 7 times the urinium content ior the average monzonite and syenite and about 4 times the thorium background. 


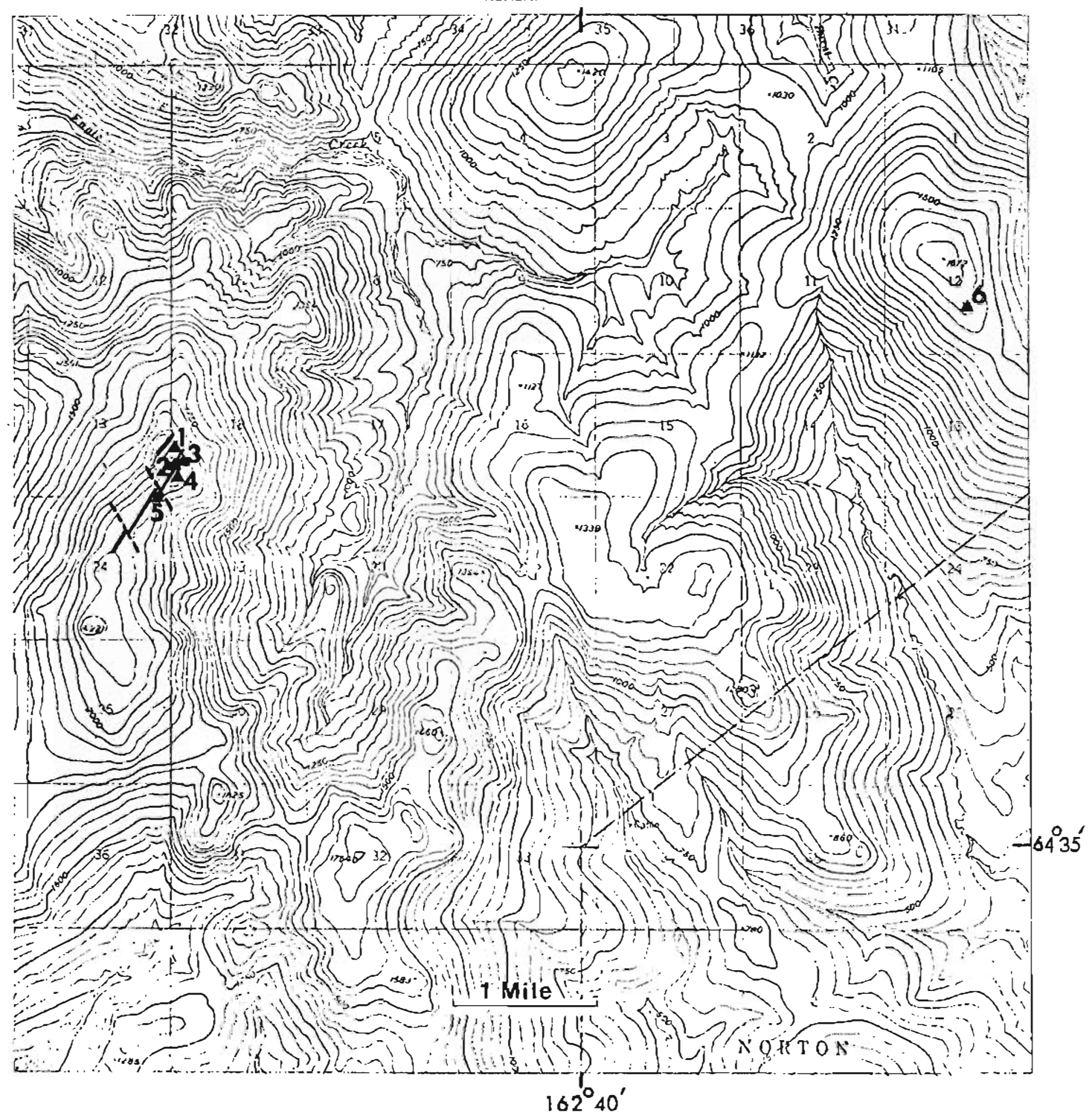

Figure 7. Location of mineralized areas and analyzed samples referred to in table. 3. See figure 6 for general location. Source: Miller, Elliott, Finch, and srooks, 1976. 
These same rocks were analyzed for rare-earth content. It should be noted that these are semi-quantitative spectrographic analyses and therefore are only approximate. The analyses, however, indicate that the rare-earth content, particulariy that of the cerium group of rare-earths, may exceed 2 percent in some samples.

On a low Elat-topped spur $1.5 \mathrm{mi}$ to the north in the $\mathrm{NW} I / 4$ of Sec. 7, T. 9 S., R. $20 \mathrm{~W}$. (fig. 7), radioactivity readings of 600 cps were recorded on the bog and tundra-covered ridge crest suggesting other mineralized areas may occur in this area.

Similar allanite-rich syenite was found occurring as isolated boulders on a largely tundra-covered ridge $10 \mathrm{~km}$ (6 $\mathrm{mi})$ to the east in Sec. 12, T. $9 \mathrm{~S} ., R .20 \mathrm{~W}$. north of Burnt Creek (Eig. 7). A fist-sized sample (no. 6, table 3) taken from a large boulder yielded $392 \mathrm{ppm} U\left(0.059\right.$ percent $\left.U_{3} \mathrm{O}_{8}\right)$ and 9,200 ppm Th $(1.05$ percent $\mathrm{ThO}_{2}$ ).

Allanite appears to be the principal uranium-, thorium-, and rare-earth-bearing mineral in the several samples examined in thinsection. A detailed study of the mineralogy has not been made, however, and other uranium minerals may be present. The allanite occurs as euhedra as much as $1.25 \mathrm{~cm}(0.5 \mathrm{in})$ long, is strongly metamict, and cut by numerous anastomosing fractures. A characteristic bright reddish-brown weathered crust is found on the weathered surfaces of many of the allanite crystals: according to Hata (1939), this material consists mainly of ferric hydroxide, alumina, silica, and carbon dioxide. Zircon, while moderately abundant, occurs only as an accessory.

The mineralized axeas and much of the area of alkaline dike occurrence are in lands presently withdrawn for native village and/ or regional corportation selections under the Alaska Native Claims Settlement Act (ANCSA) of 1971; the remainder of the area of alkaline dike occurrence is withdrawn under section $D-2$ of ANCSA for possible inclusion in national systems lands (Parks, Wildlife Refuges, etc.).

The extent and value of the mineralized areas described here is unknown owing to the relatively poor exposures and the brief time spent in the area. The width of the mineralized zones are unknown and could be quite narrow; their strike length, however, could be considerable. The uranium, thorium, and rare-earth contents of samples are sufficiently high as to indicate further study of the economic potential of the deposits is warranted.

of perhaps equal importance is the fact that similar minera.lized material was found associated with alkaline dikes in two other localities, one of which is 6 miles away from the hill 2.109 locality. These dikes axe enriched in uranium and thorium as compared to the average monzonite-syenite of the pluton and may represent a lithologic and/or structural control of the uranium, thorium, and rareearth mineralization. Since the alkaline dikes are part of a dike swarm that crops out over at least $250 \mathrm{~km}^{2}\left(100 \mathrm{mi}^{2}\right)$, this entire area appears worthy of more detailed exploration for uranium, thorium, and rare-earth elements. 
Table 3. Uranium and thorium analyses in parts per million (ppm) of samples from the Kachauik pluton. Map number refers to

Figure 7 (Miller, Elliott, Finds, Brooks, 1976.)

\begin{tabular}{|c|c|c|c|c|c|}
\hline Map no. & Field no. & $\underline{\underline{p} p m^{I}}$ & Uppm ${ }^{2}$ & Th ppml & Th ppm ${ }^{2}$ \\
\hline 1 & $76 A M m I J 2$ & 1.107 & 1000 & 6619 & 5700 \\
\hline 2 & $76 A M m I 12 C$ & 34 & $-\quad-$ & 96 & - \\
\hline 3 & $76 \operatorname{AEr} 23$ & 2162 & 1050 & 7692 & 6400 \\
\hline 4 & $76 A M 212 B$ & 1486 & - & 9240 & - \\
\hline 5 & $76 A E r 23 B$ & 1545 & 1500 & 8408 & 7000 \\
\hline 6 & 76 AMmIIO & -- & 392 & - & 9200 \\
\hline
\end{tabular}

IDelayed neutron determination. The coefficient of variation of uranium and thorium is more than $1 \%$ for all samples. Analysts A.J. Bartel and R.J. Vinnola.

${ }^{2}$ Camma-ray spectrometric analysis. The coefficient of variation of uranium and thorium is more than $2 \%$ for all samples. Analyst.s C.M. amber inm in. Ansh.

Aerial Radiometric Survey

The aerial radiometric survey produced a "perferred" anomaly over the quartz monzonite of the Darby pluton in the northern part of the Solomon A-l quadrangle (flight-line 71), and over the granodiorite of the Kachauik pluton (flight-line 73). Three suspect anomalies were calculated from the survey: one each over the Kachiauk and Darby plutons and one over Cretaceous conglomerate on the northeast flank of the Darby Mountains.

Ground Radiometric Survey

Results of the ground radiometric survey are shown in figures 8 and 9. The readings were high even for alkaline intrusive rocks: 200 to 600 cPs over most of the upland area, compared to about 50 cps in the surrounding lowlands. The maximum reading, I,200 cps, was obtained at stream-sediment sample location $\mathbf{3 9 2}$ in one of the uppermost reaches of the Kwiniuk River. The 1,200-cps count was obtained at a point in sandy and gravely loam on the bank of a small stream. A soil sample at the point yielded $9 \mathrm{ppm} U$ and $90.8 \mathrm{ppm} T \mathrm{Th}$. Bedrock was not exposed, but the area is underlain by granodiorite of the Kachauik pluton.

The Dry Canyon pluton on the west flank of the Darby Mountains in the Solomon D-2 quadrangle is anomalously radioactive - 400 to 700 cps. The radiometric survey was supported by unusually high $U$ and $T h$ in bedrock and stream-sediment samples. A flight line of the aerial radiometric survey passed over the northern end of the pluton. Three consecutive data points recorded only $1-2$ standard deviations and an aerial radiometric anomaly was not reported.

Suggestions for Exploration

Above-average $U$ and $T h$ in stream-sediment and water samples, and a broad radiometric anomaly in the headwaters of Vulcan and clear Creeks of 


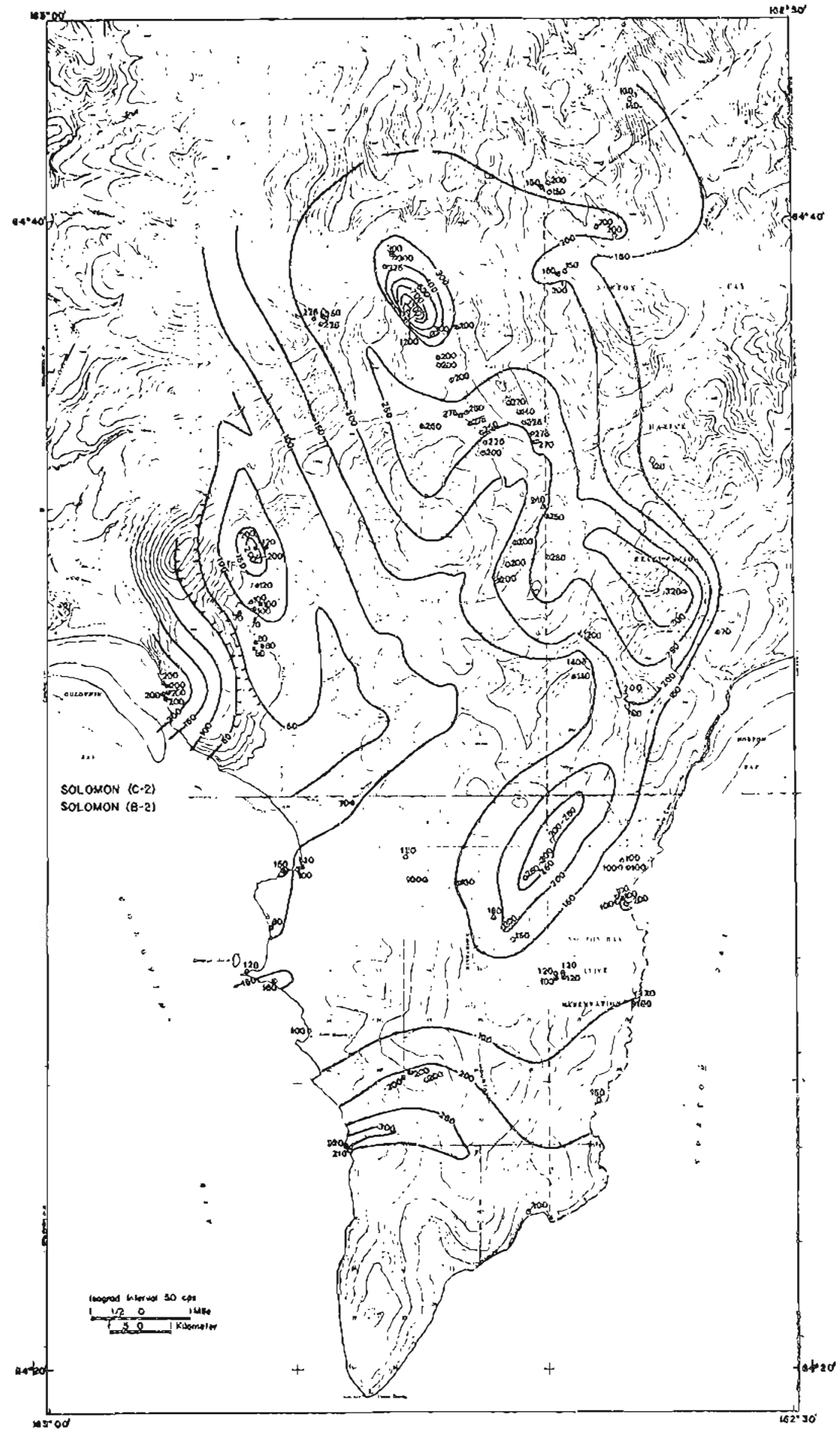

Figure 3. Ground radiometric survey, southern Darby Mountains area, Solomon $\mathrm{B}-2$ and $\mathrm{C}-2$ quadrangles; by G.R. Eakins and C.L. Carver, 1976. 


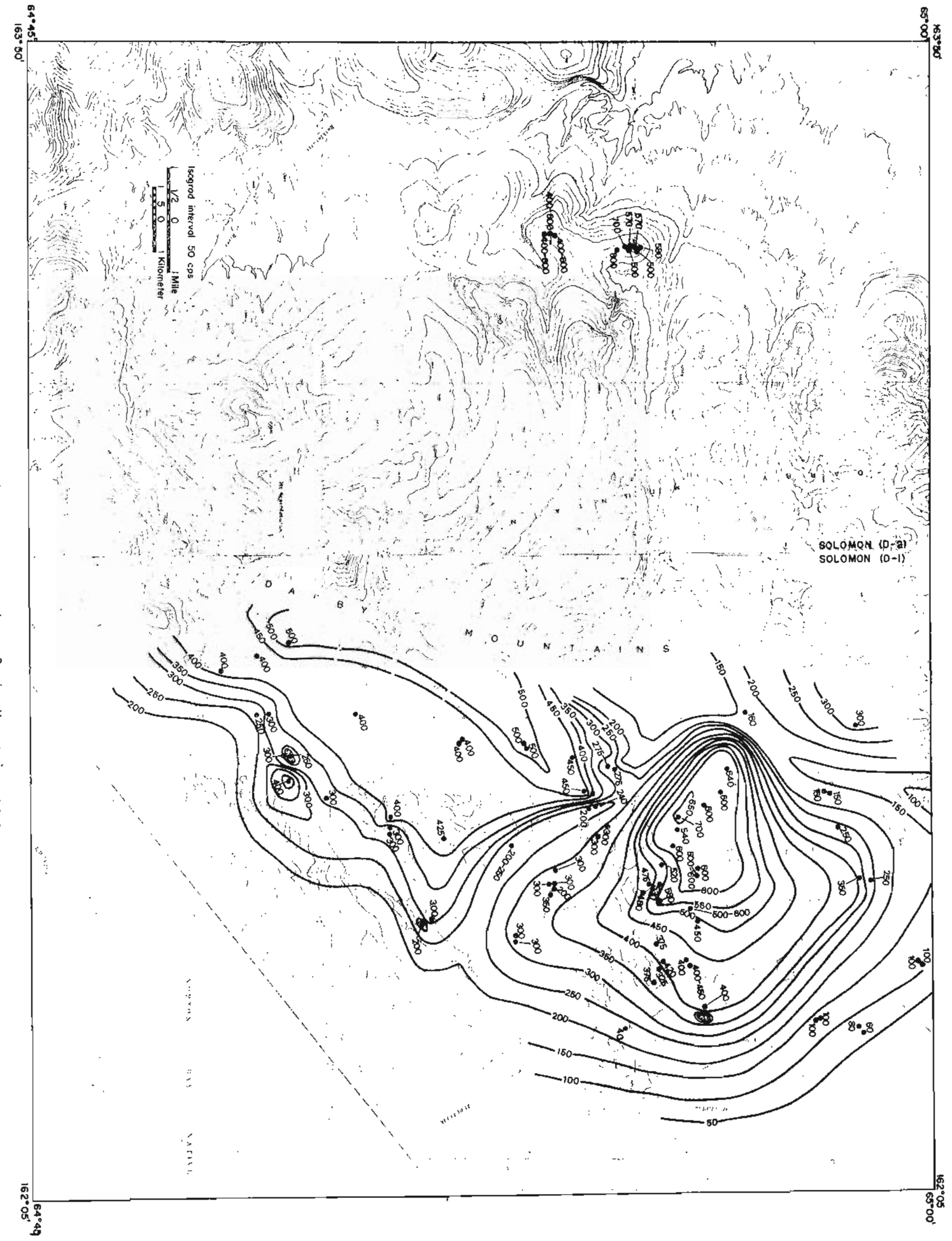


the centra! and east-central Darby Mountains may indicate a favorable area for uranium exploration. Follow-up work is also suggested in the vicinity of sediment sample $J 92$ and in the headwaters of the Kwinik River, where a scintillometer reading of 1,200 eps was obtained. Examination of the Dry Canyon pluton is considered warranted on the basis of sediment samples and the ground radiometric survey.

The highly radioactive alkaline dikes in the western part of the area, which contain up to 0.15 percent $U_{3} \mathrm{O}_{8}$ and 1.05 percent Th as reported by the USGS, are very encouraging finds in this region and indicate the need for detailed investigations.

\section{Selawik Hills Area}

The Selawik Mills (pi. 3) lie in the southern part of the Selawik quadrangle and along the northern edge of the Candle quadrangle. The field party was based at the Native village of Buckland on the Buckland River during the 1 week in the vicinity. Sampling and radiometric surveys were concentrated on the alkaline rocks of the Selawik Hills pluton and the small Inland Lake and Selawik Lake plutons that lie a short distance north of the Selawik Hills (fig, 2). The geologic setting is described in appendix E under "Hogatza Plutonic Belt of Westcentral Alaska." The Selawik Hills area has already attracted uranium explorationists. During the summers of 1975 and 1976, the Wyoming Minerals Corporation, a subsidiary of Westinghouse, conducted a drilling program in the central part of the Selawik Hills in the Selawik A-3 quadrangle. Results are not available to the writer, but it was rumored that deep holes were orilled to test the pluton for secondary enrichment at depth.

Stream-Sediment Sampling

A total of 84 stream-sediment samples were collected. Results of the analyses are:

\begin{tabular}{|c|c|c|c|c|}
\hline & Range & Mean & Threshold & Anomalies \\
\hline Uppm (RAA) & $0.0-27.4$ & 5.3963 & 13.8971 & 3 \\
\hline Uppm (LASL) & $1.80-100$ & 13.3966 & 44.6608 & 3 \\
\hline Th ppm & $1.0-96.9$ & 31.9267 & 79.8525 & 2 \\
\hline $\mathrm{K}_{2} 0 \%$ & $0.93-7.23$ & 2.6755 & 4.8203 & 2 \\
\hline
\end{tabular}

There is a considerable descrepancy between the RAA and LASL analyses. Los Alamos did not receive all the samples, but those they did analyze were nearly all notably above the average for $U$ in alkaline intrusive rocks. Except for samples 666-681, most of the uranium values reported by RAA were not unusual. These 15 samples were collected in the Selawik A-4 quadrangle, in the western part of the Selawik Hills. However, the analyses by Los $A 1$ amos showed above normal $U$ to be much more widespread; 28 samples contained over $10 \mathrm{ppm}$. The higher $U$ values reported by Los Alamos seem more consistent with the high radiometric background and high Th values.

Water Sampling

Uranium values from 57 water samples collected in the Selawik Hills 
ranged from 0.00 to $1.75 \mathrm{ppb}$. The results are surprisingly low for an area of known high radioactivity:

$\mathrm{H}_{2} \mathrm{O}(\mathrm{ppb}) \quad \frac{\text { Maximum }}{1.7000} \quad \frac{\text { Mean }}{0.4962} \quad \frac{\text { Threshold }}{1.2276} \quad \frac{\text { Anomalies }}{5}$

Only six of the water samples exceeded I ppb. Four of these (675G78) were collected from the southwestern flank of the Selawik Hills, south of the center of the Selawik $A-4$ quadrangle. The corresponding stream-sediment samples are above average for both $U$ and Th.

\section{Bedrock Sampling}

A total of 66 bedrock samples from the Selawik area were analyzed. Results are:

\begin{tabular}{|c|c|c|c|c|}
\hline nam & Range & $\frac{\text { Mean }}{108823}$ & Threshold & $\frac{\text { Anomal i es }}{3}$ \\
\hline Th ppm & $0.0-618$ & 46.0958 & 239.2816 & 3 \\
\hline $\mathrm{K}_{2} 0 \%$ & $0.0-17.05$ & 7.7311 & 15.3131 & 3 \\
\hline
\end{tabular}

Rock samples GIR-GIOR, collected from the Inland Lake pluton in the Selawik B-3 quadrangle, suggest a potential uranium area. This poorly exposed alkaline complex underlies a group of low hills located 6 to 10 miles north of the Selawik Hills. The rocks, according to Miller (1972, table 2) include pulaskile, malignize, foyaite, nepheline syenite, and alaskite. Two samples of the group, $G 8 R$ and $G I O R$, contained unusual amounts of $1,86.0$ and $92.0 \mathrm{ppm}$, respectively. The corresponding Th values are 70.3 and $37.0 \mathrm{ppm}$, respectively. An outstanding characteristic of the pluton is the exceptionally high $K_{2} 0$ content, 10.65 to 17.19 percent.

Two other bedrock samples with unusual $U$ and $T$ h contents are samples $953 \mathrm{R}$ and $\mathrm{G} 54 \mathrm{R}$, which contain 139.0 and $44.0 \mathrm{ppm} U$, and 613.0 and 277.5 ppm Th, respectively. These samples were obtained from an altered felsic dike that is poorly exposed on the southeast side of a low spur (hill 860) in sec. $30, R .8 \mathrm{~N} ., T .9 \mathrm{~W}$. near the center of Selawik A-4 quadrangle. The dike, possibly a syenite, was found to be highly radioactive and produced up to $2,000 \mathrm{cps}$ on the outcrop. One radioactive mineral was identified by the DGGS laboratory as tantalian rutile (struverite; Fe [Ta, Nt] Tio) which occurs in small ( $1 \mathrm{~mm}$ ) crystals. The dike is about 3 feet wide and is exposed at three points within a distance of 200 feet. The strike is northwest. Radioactivicy of adjacent igneous rocks along the crest of the spur to the west is also high (400 to $600 \mathrm{cps}$ ).

While an extension of the radioactive dike was sought along a projection of the strike to the southeast, it was not found. However, a highly anomalous stream-sediment sample (G73) collected 1.1 miles southeast of the dike outcrop and possibly on strike was found by the Los Alamos laboratory to contain $100 \mathrm{ppm} U$. RAA reported $27.4 \mathrm{ppm} U$.

The group of four anomalous water samples described under "Water Sampling," above, were collected about 3 miles to the southwest of these 
bedrock and sediment samples. Although the samples were found in different drainages, their near proximity may be significant and add evidence for a possible $U$ concentration in the general area.

Aerial Radiometric Survey

Texas instruments reported two preferred anomalies over the Selawik Hills: one on the north slope on flight-line 57 in the selawik A-3 quadrangle and one on $f$ light-line 58 over the southeastern part of the Selawik A-2 quadrangle. A suspect anomaly was shown at the intersection of flight-lines 57 and 457 in the southwest part of A-4 quad.rangle. Spacing of the flight lines does not permit correlations with sampling results.

\section{Ground Radiometric survey}

Results of ground radiometric investigations in the Selawik Hills area are shown in figures 10, 11 , and 12 . The general background was found to be relatively high over the plutonic rocks, averaging possibiy $300 \mathrm{cps}$ (compared with a low of $30 \mathrm{cps}$ encountered at the edge of the Selawik Hills). Two radiometric highs are conspicious in the A-4 quadrangle: one shows a maximum value of $700 \mathrm{cps}$ near the summlt of a 1,360foot hill in the north-central part, and one surrounding the radioactive dike mentioned under "Bedrock Sampling," above. Another high appears as a broad anomaly in the central part of the Selawik A-3 quadrangle.

\section{Suggestions for Exploration}

Much of the Selawik Hills area could not be sampled or examined even in a cursory manner within the time allowed for this part of the program. in light of the $U$ and Th values found in the samples and the anomalous radioactivity, however, it is believed that more complete investigations can produce additional anomalies and define drilling targets. Forbes and Jones found that uranium is associated with biotite in this area.

The radioactive dike and adjacent bedrock in the central part of the Selawik A-4 quadrangle, the nearby sediment-sample containing 100 ppm $U$, and the relatively high $U$ content in water samples $3-4$ miles to the south suggest a several-square-mile area that warrants detailed study. The broad radiometric anomaly and moderately high stream-sediment samples in Selawik A-3 quadrangle also indicate a favorable area for exploration; this area includes Wyoming Minerals' claims. Wyoming Minerals reportedly has drilled the intrusive rocks to depths of severai hundred feet in an effort to determine if there has been secondary enrichment of $U$, but no data are available to the writer.

The highly potassic stocks in the Selawik lowlands contain as much as $92 \mathrm{ppm} U$ in dikes; this suggest that detailed study in this locality is warranted.

The Zane Hills Area

The DGGS field party was based near UV Industries' gold dredge camp at Hogatza on Bear Creek while conducting field work in the Zane Hills 


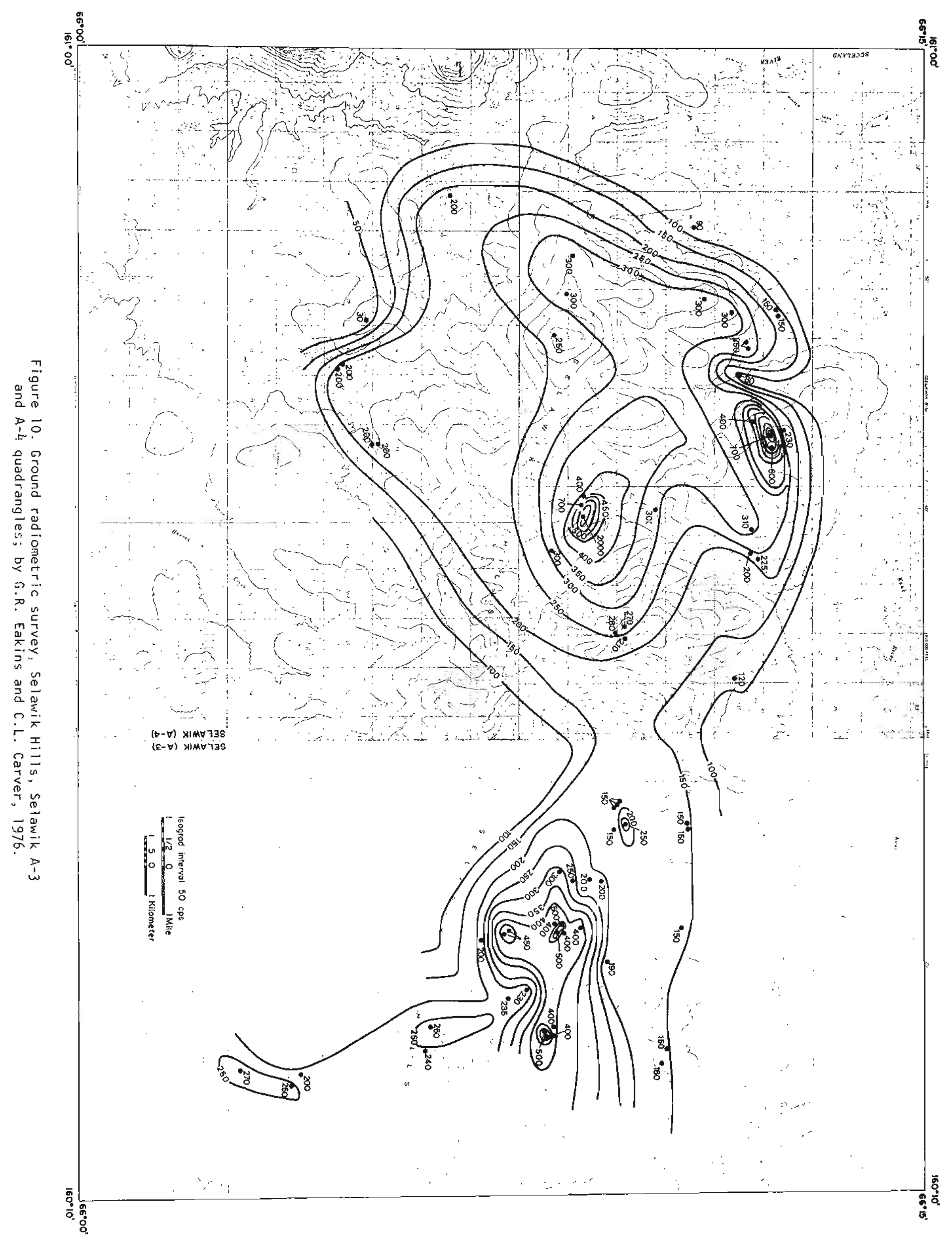




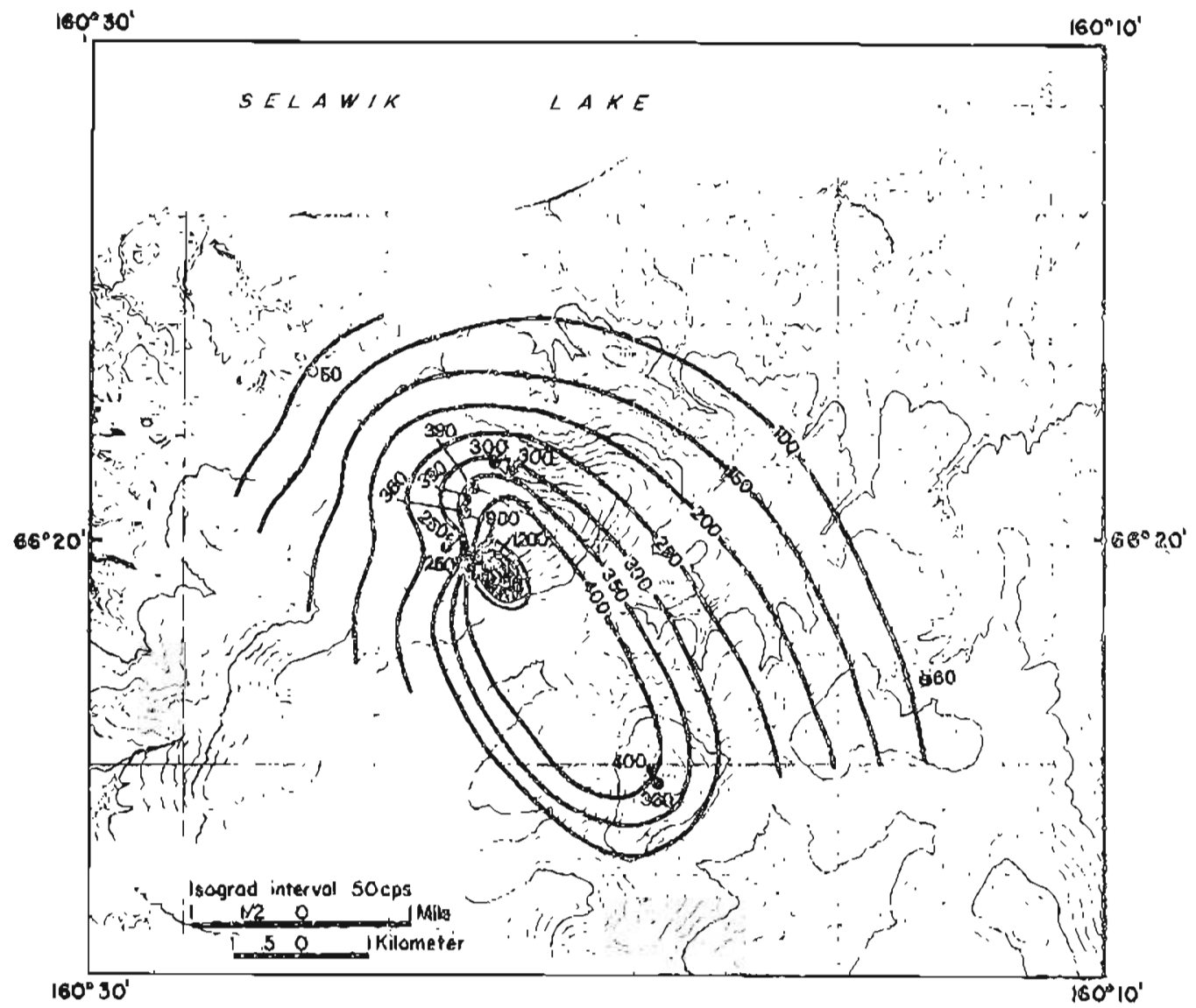

Figure 11. Ground radiometric survey, western Selawik Hills, Selawik B-3 quadrangles; by G.R. Eakins and C.L. Carver, 1976.

and Purcell Mountains ( $p$ l. 4). The area lies in the Hughes and Shungnak quadrangles (fig. 2). There is a good landing strip at Hogatza, but it is privately owned and permission must be obtained prior to use.

Anomalous amounts of $U$ in the quartz monzonite border phase of the Zane Hills and mineral occurrences in both the Zane Hills and Purcell Mountains have been known for some time (Miller and Ferrians, 1968) and the region is considered favorable for uranium exploration. A summary of the geology is included in appendix $E$, under "Hogatza plutonic belt of west-central Alaska," and the petrology is discussed by Forbes and Jones in the section on granitic rocks.

Parts of the Cretaceous Zane Hills and Wheeler Creek plutons were sampled ( $\mathrm{pl}$. 4). The Zane Hills pluton is predominantly granodiorite, but monzonite and quartz monzonite constitute about 10 percent of the rocks. The Wheeler Creek pluton, which forms a large part of the Purcell Mountains, is composed of granodiorite, quartz monzonite, and alaskite.

\section{Stream-Sediment Sampling}

A total of 96 stream sediments were collected in the. Zane $\mathrm{Hill}$ s area. The values from the analyses are: 


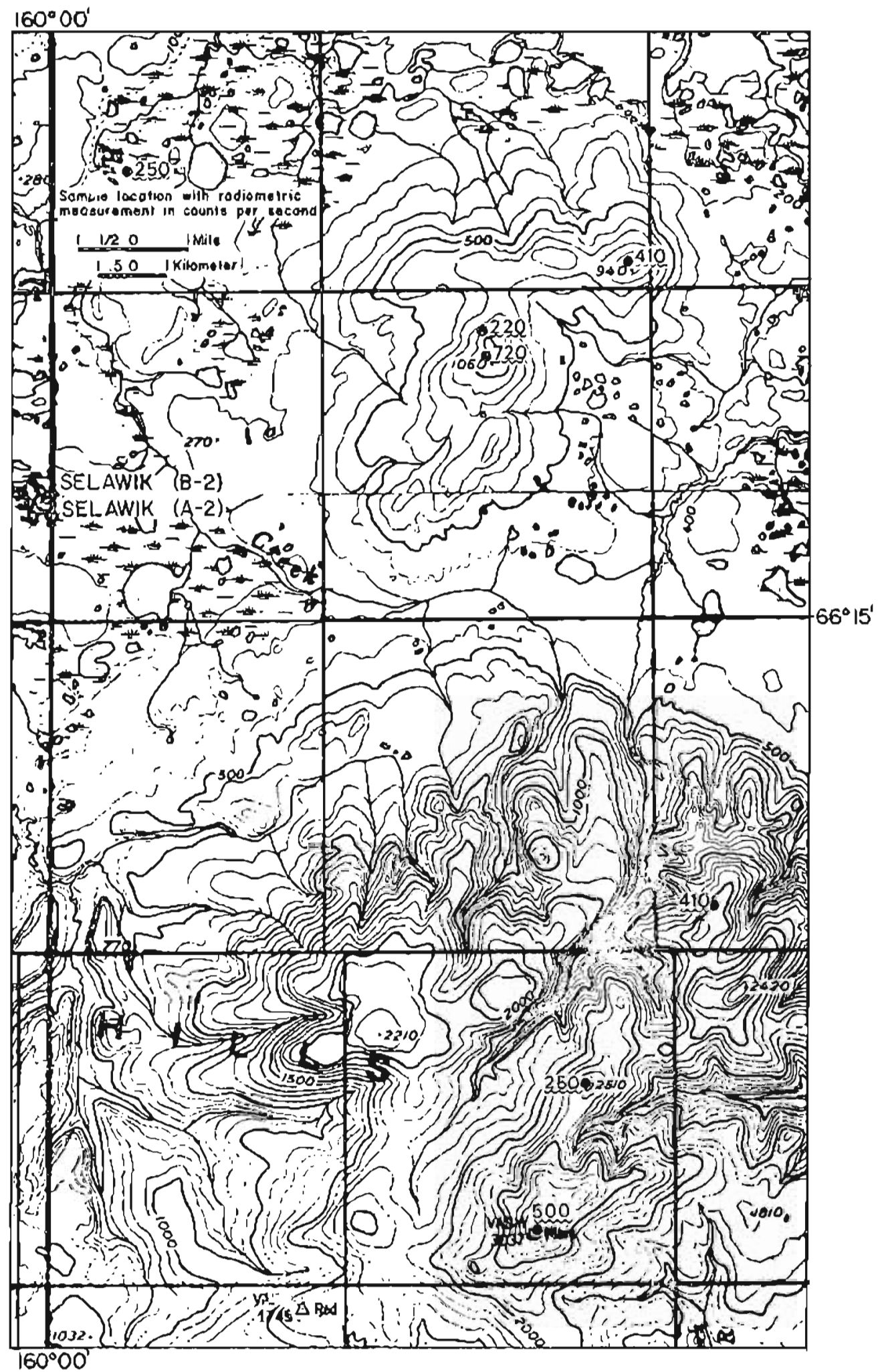

Figure 12, Ground radiometric survey, Selawik Hills-|nland Loke area, Selawik A-2 and B-2 quadrangles: by G.R. Eakins and C.L. Carver, 1976. 


\begin{tabular}{|c|c|c|c|c|}
\hline & Range & Mean & Threshold & Anonialies \\
\hline Uppm (RAA) & $0.8-57.0$ & 11.7126 & $\overline{35.3198}$ & 6 \\
\hline Uppm (LASL) & $3.3-77.0$ & 22.3518 & 59.0502 & 7 \\
\hline Th ppm & $4.5-120.0$ & 30.2095 & 72.6465 & 5 \\
\hline $\mathrm{K}_{2} 0 \%$ & $0.97-3.69$ & 2.0772 & 3.245 & 4 \\
\hline
\end{tabular}

The $U$ analyses reported by Los Alamos average over 50 percent above those reported by RAA, but both sets of analyses contain numerous relatively high values.

The border zones along the southeastern-southwestern edges of the Zane Hills yielded stream sediments with contents higher than those found in other parts of the pluton, $U$ up to $60 \mathrm{ppm}$. Thi up to $98 \mathrm{ppm}$. These areas have been described by Miller and Ferrians (1968).

A second particularly anomalous area is the western part of the Wheeler Creek pluton (samples $\mathrm{H3} 3-\mathrm{H} 39$ and H52-H57) in the Purcell Mountains. The area lies in the Shungnak $A-3$ and $B-3$ quadrangles. Uranium was as much as $77.0 \mathrm{ppm}$ and Th as much as $120.0 \mathrm{ppm}$. The anomalous area lies across the contact of alaskite on the west and monzonite and quartz monzonite on the east.

Water Sampling

A total of 84 water samples from the Zane Hills and Purcell Mountain were analyzed for $U$.

$\mathrm{H}_{2} \mathrm{O}$ ppb $\frac{\text { Maximum }}{3.95} \quad \frac{\text { Mean }}{1.1220} \quad \frac{\text { Threshold }}{2.669} \quad \frac{\text { Anomalies }}{5}$

The nine samples in the series $030-038$ compose an anomalous group. These samples were collected from the Caribou Creek drainage in the southern part of the Zane Hills. Sometimes they support the $U$ values reported from stream sediments and bedrock, but in other cases there is no correlation. The $U$ value of $3.95 \mathrm{ppb}$ is the highest obtained from any water samples collected during the entire 1975 DGGS project.

Bedrack Sampling

A total of 49 bedrock samples were collected from the Zane Hills and Purcell Mountains. Their analyses produced the following values:

\begin{tabular}{|c|c|c|c|c|}
\hline & Range & Mean & Threshold & Anomalies \\
\hline $\mathrm{U} p \mathrm{ppm}$ & $0.60-49.0$ & $\overline{6.6872}$ & 24.632 & 2 \\
\hline Th ppm & $2.08-126.3$ & 27.5588 & 73.9118 & 2 \\
\hline$K_{2} 0 \%$ & $0.75-8.15$ & 4.1629 & 7.3887 & 1 \\
\hline
\end{tabular}

Eleven of the 49 rock samples were noticeably above average for granitic rocks (over $6 \mathrm{ppm}$ ), but the remaining seem low for this area. This may be partly due to incomplete extraction of $U$ from silicate accessory minerals. None of the calculated anomalies for the three eiements is for the same sample. The U:Th ratio exceeds 3.6 in one sample, H4R. 
Anomalous $U$ was found in pegmatite dikes. Two samples, $H 4 R$ and $H 5 R$, yielded 49.0 and $19.4 \mathrm{pom} U$, respectively. These are from the east side of Zane Hills near the contact with the volcanic rocks on a spur 14.5 miles northwest of Hogatza.

Samples H3OR-H38R are noticeably high in $U$. These samples represent alaskite from the western end of the Wheeler Creek pluton in the Purcell Mountains. The $U$ values for six rocks of this group range from 9.0 to $32.7 \mathrm{ppm}$.

\section{Aeria1 Radiometric Survey}

The aerial survey by Texas Instruments produced a preferred anomaly over Caribou Mountain on the east side of the Zane Hills, 4 to 8 miles west of Hogatza. Five bedrock samples from this locality (D5R-D9R) were collected by the DGGS party, but none was anomalous in $U$, Th, or $k_{2} O$. A ground radiometric anomaly of $600 \mathrm{cps}$, however, was measured on the east side of Caribou Mountain peak.

A broad aerial radiometric anomaly was produced over the central part of the Purcell Mountains at the junction of flight-lines 56 and 461 . No samples were collected within the anomalous area, but anomalous stream sediments ( $\mathrm{H} 42, \mathrm{H} 53$ ) were obtained about 3 miles to the west.

Several suspect anomalies were calculated in the Hughes and Shungnak quadrangles, but their significance is questionable. Some appear over tundra-covered lowlands.

Ground Radiometric Survey

The radiometric background of the Zane Hills pluton is generally high (figs. 13, 14, and 15); the average was found to be $200 \mathrm{cps}$ or more. Radiometric highs are present on Caribou Mountain (600 cps) and across the southern end of the Zane Hills south of Caribou Creek, where radiometric readings were as much as 1,000 cps. A broad area in the central part of the Purcell Moutains is a radiometric high where counts are 400 to $500 \mathrm{cps}$.

Correlation of radiometric readings with the $U$ and Th contents of stream sediments and rock samples is usually good, but sometimes poor. Again, the incomplete extraction from some of the bedrock samples is a possibility.

\section{Suggestions for Exploration}

Anomalous $U$ and Th values were obtained from stream-sediment, bedrock, and water samples in the Zane Hilis and Purcell Mountains, and radiometric highs are present. The anomalous localities warrant more detailed sampling, and the areas not sampled by the DGGS should also be investigated. The broad geochemical and radiometric anomalous part of the Purcell Mountains alaskite seems to offer promise for a uranium deposit. Forbes and Jones recommend concentrating on the augen gneiss unit which they discovered.

The adjacent lowlands of the Pah River Flats and the Selawik and Koyukuk Rivers may deserve study for possible sedimentary-type uranium deposits. 


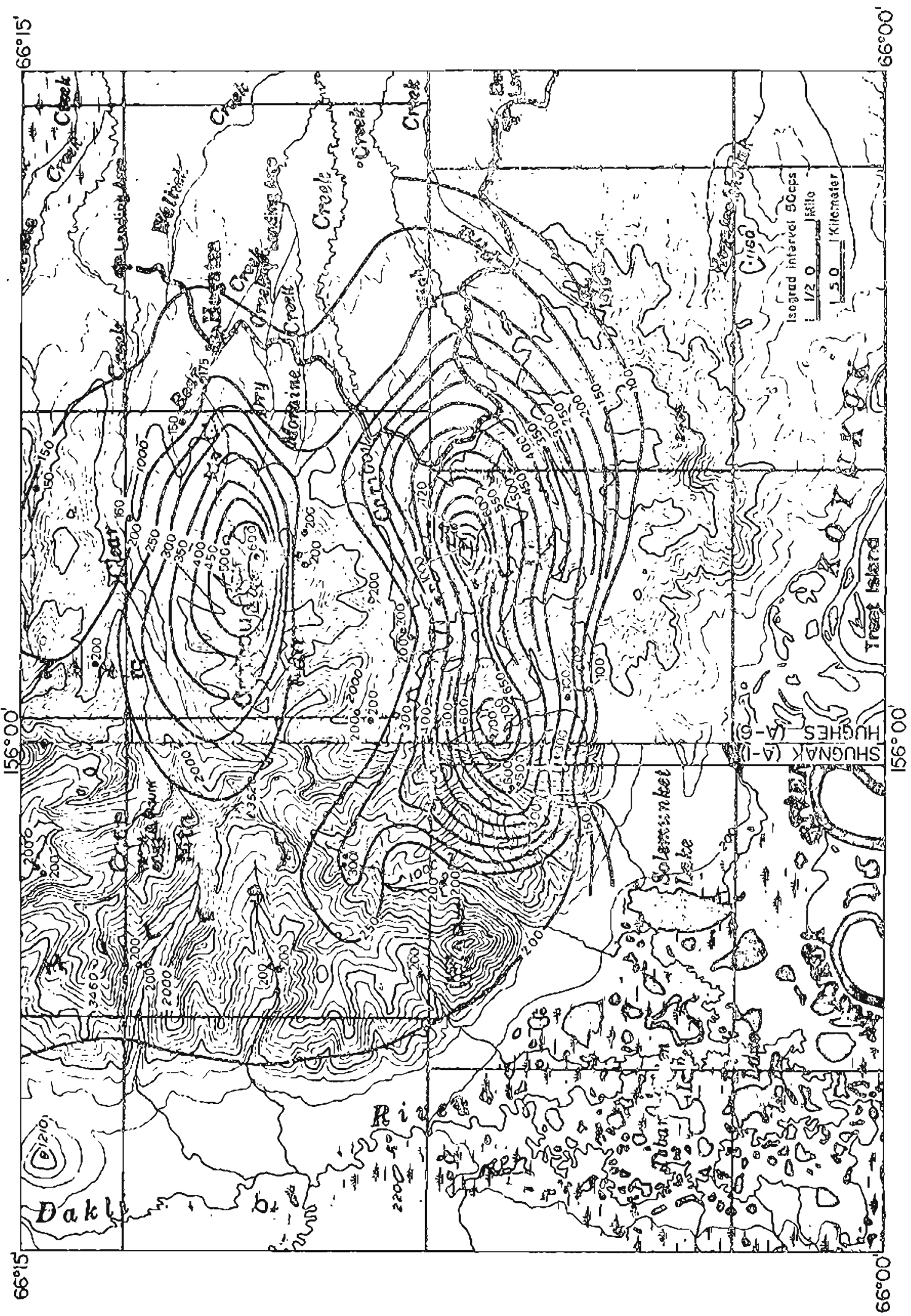

Figure 13. Ground radiometric survey, southern Zane Hills, Shungnak A- 1 and Hughes A-6 quadrangles; by G.R. Eakins and C.L. Carver. 1976. 


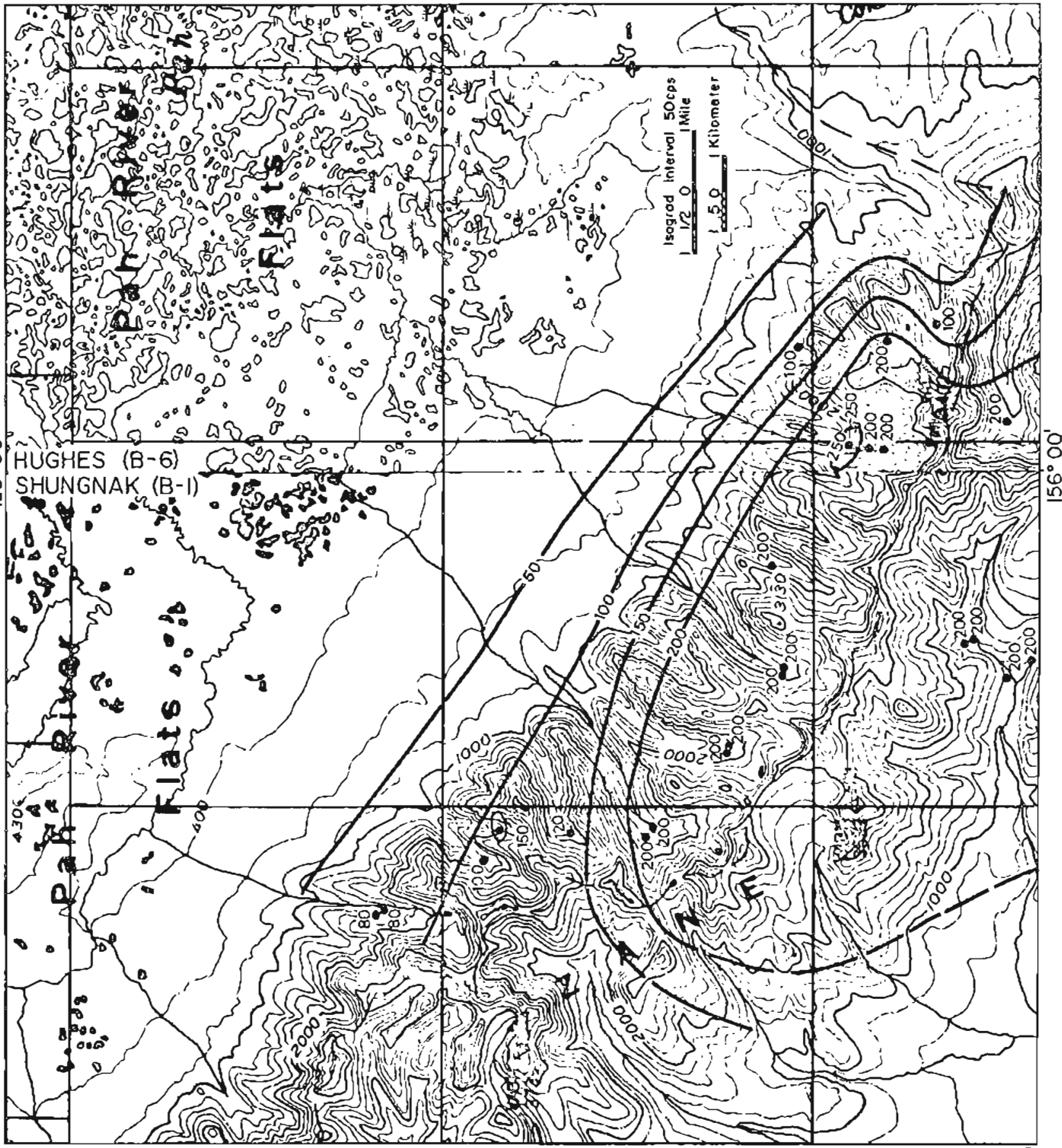

Figure 14. Ground radiometric survey, northern Zane Hills, Shungnak B-I and Hughes B-6 quadrangles; by G.R. Eakins and C.L. Carver, 1976. 


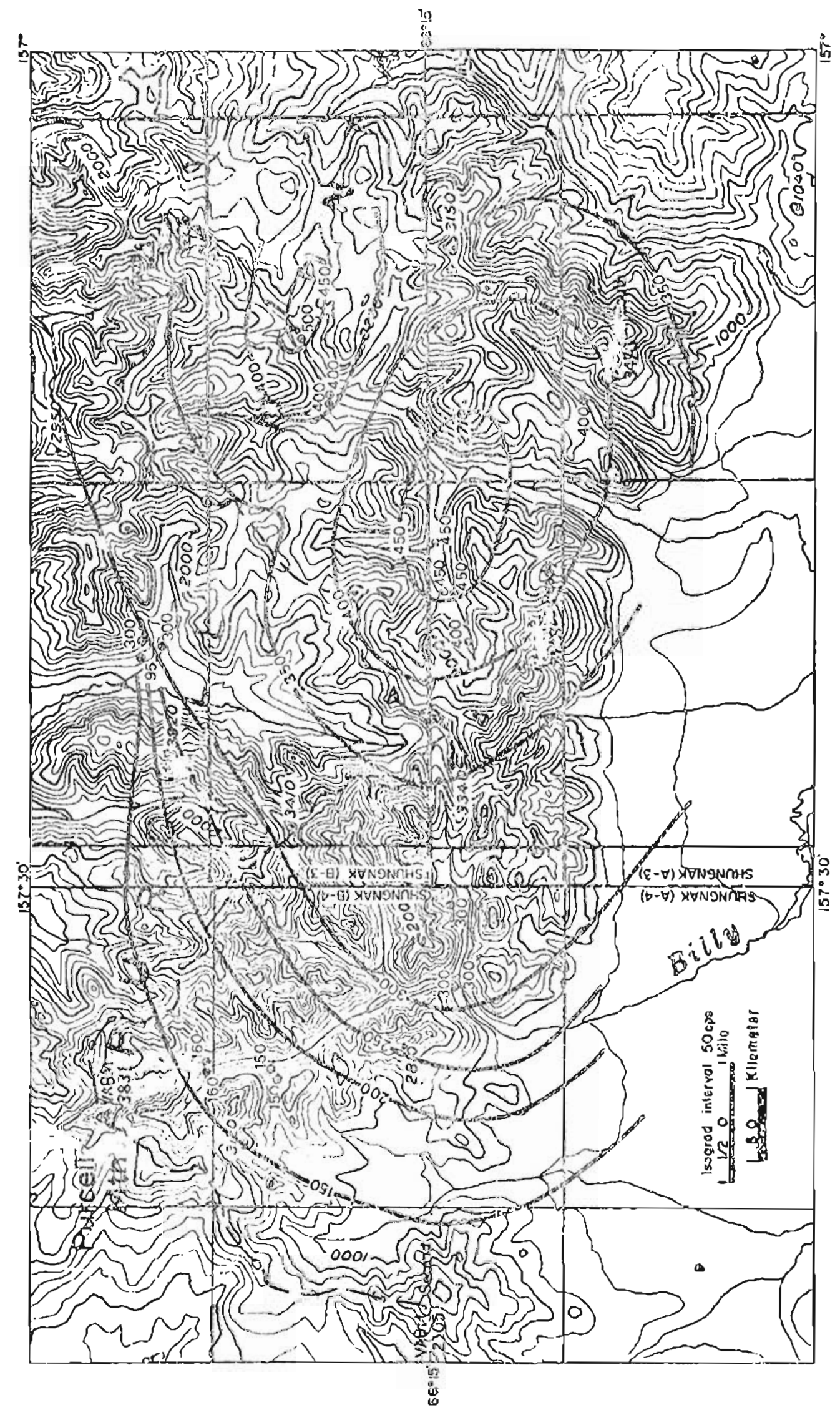

Figure 15. Ground radiometric survey, Purcell Mountains, Shugnak $A-3, A-4, B-3$, and $B-4$ quadrangles; by $G, R$. Eakins and C.L. Carver, 1976. 
The Copper River basin is a confined area about 80 miles across with narrow outlets. It is filled with a variety of glacial and fluvial sediments of Cretaceous and Tertiary ages that are considered possible hosts for sedimentary $U$. The area lies in the Gulkana and eastern part of the Talkeetna Mountains quadrangles (fig. 16). The Cretaceous and Tertiary sediments are poorly exposed, but samples of Tertiary sandstones were obtained along the Richardson Highway in the northwestern part of the basin and near the Glenn Highway in the southeastern part. Areas of intrusive rocks in the Talkeetna Mountains along the western margin of the basin were sampled to determine their favorability as host to vein deposits or sources for sedimentary deposits. The geology is discussed in appendix E under the section on the Copper River basin-Chitina River valley. Minor exploration activity for petroleum and uranium by industry was still being conducted during 1975 and 1976.

Field work was conducted from a lodge on the Glenn Highway with helicopter supporl during a 5-day period. Sample location sites appear on plates 5, 7, and 10 .

Stream-Sediment Sampling

In the Copper River basin area, 96 stream-sediment samples were collected. Statistics of their analyses are:

$\begin{array}{lllcc} & \frac{\text { Range }}{30-80} & \frac{\text { Mean }}{1.30} & \frac{\text { Threshold }}{2.45} & \text { Anomalies } \\ \text { Uppm (RAA) } & 0.30-2.80 & 1.44 & 2.80 & 3 \\ \text { Uppm (LASL) } & 0.15-3.30 & 1.29 & 9.71 & 5 \\ \text { Th } \rho p m & 0.50-16.0 & 4.29 & 1.60 & 2 \\ \mathrm{~K}_{2} \mathrm{O} \% & 0.20-2.00 & 0.97 & \end{array}$

II and Th values in all samples were consistently low, especially when compared with those collected in west-central Alaska. One anomalous sample (C5) was from Cache Creek in the southern part of calkana A- 6 quadrangle in an area underlain by Tertiary or Mesozoic sediments. The other three anomalous sediment samples were from scattered locations in the headwaters of the Susitna River (Talkeetna Mountains B-2 and C-2 quadrangles) in areas underlain by Mesozoic and older volcanic and sedimentary rocks. The low values and a lack of clustering of the anomalous results prevent the outlining of good target areas.

Water Sampling basin:

Water samples were collected from 74 locations in the Copper River

$\mathrm{H}_{2} \mathrm{O} \mathrm{ppb} \quad \frac{\text { Range }}{0.25-1.95} \quad \frac{\text { Mean }}{0.91} \quad \frac{\text { Threshold }}{1.74} \quad \frac{\text { Anomalies }}{3}$

The three $U$ anomalies are from scattered locations. One of the three anomalies ( $\mathrm{C} 6$ ) is from an anomalous stream-sediment location at slide Mountain and may be significant. Anomalous sample $\mathrm{k} 82$ was from the headwaters of Tyone Creek in the northeast part of the Talkeetna 


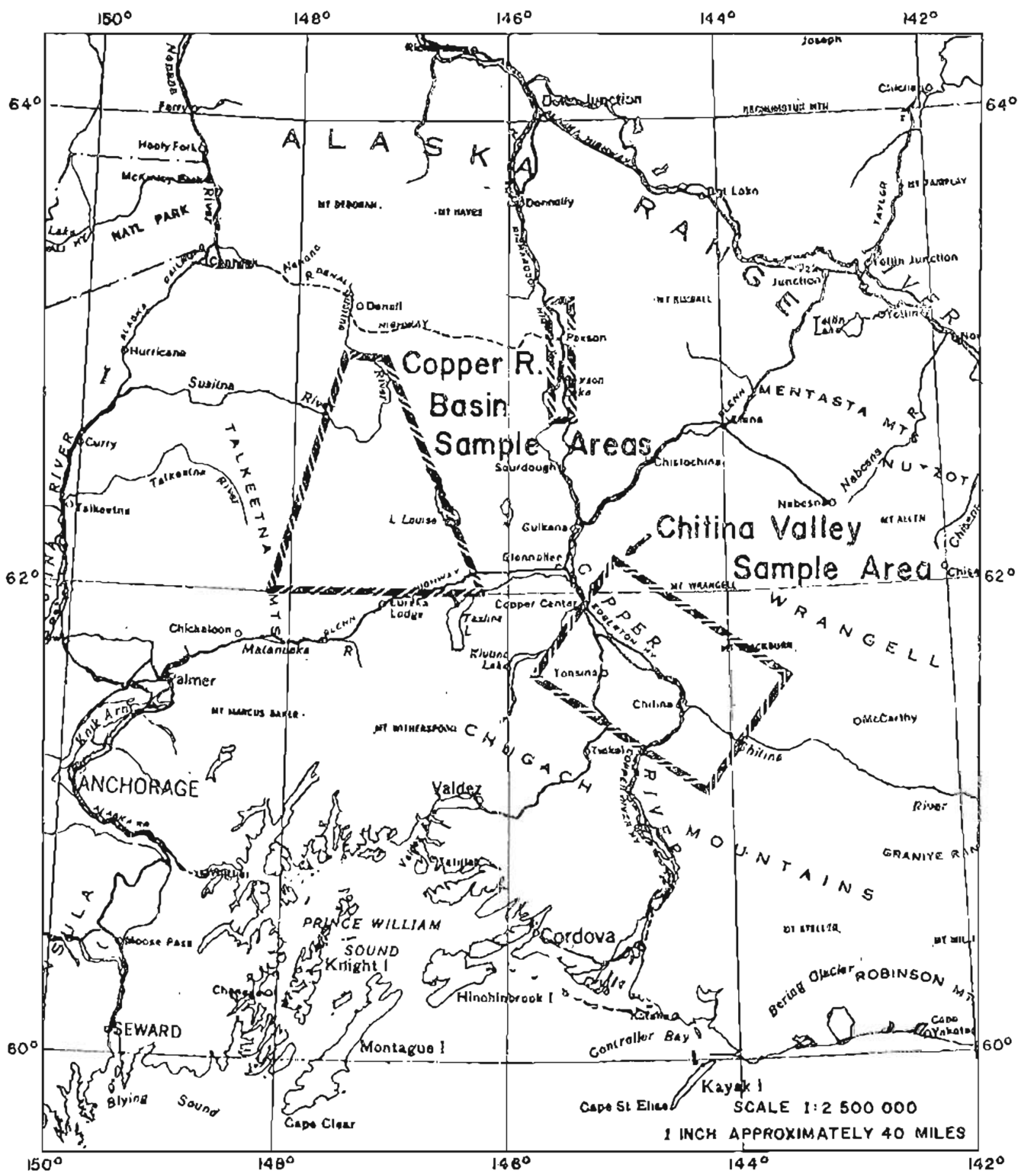

Figure 16. Index map of the Copper River basin and Chitina River valley areas, south-central Alaska. 
Mountains A-l quadrangle. This locality is underlain by Cretaceous and Tertiary sediments. The third sample (K88) was collected on a tributary to Mazuma Creek in the southwestern corner of Talkeetna Mountains A-2 quadrangle. This is in an area of Tertiary volcanics.

Bedrock Sampling

Fifteen intrusive rock samples from the Copper River basin were analyzed for $U, T h$, and $k_{2} O$. These included a variety of granites and quartz diorite. The values obtained from the analyses are:

\begin{tabular}{|c|c|c|c|c|}
\hline & Maximum & Mean & Threshold & Anomalies \\
\hline$U \mathrm{ppm}$ & 1.20 & $0 . \overline{76}$ & 1.46 & 0 \\
\hline Th ppm & 26.0 & 3.77 & 17.45 & 1 \\
\hline $\mathrm{K}_{2} 0 \%$ & 2.70 & 1.07 & 2.56 & 1 \\
\hline
\end{tabular}

The unusually low $U$ and Th values are disappointing and seem abnormally low for the types of rocks sampled. No $U$ anomalies were found in this group and only one each for $T h$ and $k_{2} O$. The low radiometric responses produced by rocks in the area was the reason so few were submitted for chemical analyses.

\section{Aerial Radiometric Survey}

The aerial radiometric survey conducted during 1975 covered the Gulkana quadrangle and the eastern part of the Talkeetna Mountains quadrangle. On plate 5 , one preferred and two suspect anomalies appear on flight-line 93, two preferred anomalies on flight-line 95, and one on flight-line 578.

Sampling by the DGGS party, preliminary results of the ERDA fallow-up study in 1976, and a lack of $U$ or Th anomalies from ground surveys indlcate that these weak aerial radiometric anomalies have little significance in this area. It is reported that at least one industry exploration party also examined the aerial radiometric anomalies produced by the Texas Instruments survey in the Copper River basin, with negative results.

Ground Radiometric Survey

Radioactivity of all types of rocks in the area examined by the DGGS party was at a very low level. Readings taken at numerous intrusive and sedimentary bedrock exposures and sample sites with hand-carried scintillometers ranged from a low of 20 to a maximum of $70 \mathrm{cps}$. The highest count was detected on Tertiary sediments on Slide Mountain in the Gulkana A-6 quadrangle. These readings are lower than those generally found to be average backgrounds in most regions, and they showed so little variation that no radiometric maps are included in this report.

\section{Suggestions for Exploration}

No targets for $U$ exploration were found in the limited areas examined in the Copper River basin or eastern Talkeetna Mountains by either the OGGS party or the aerial radiometric survey. Possibly the most anomalous area was that on the east and north flanks of Slide Mountain in the Gulkana A-6 quadrangle (samples $(3-C 6)$. The area is underlain by Tertiary and Mesozoic sediments. 
Mesozoic and Tertiary nonmarine sediments in the subsurface may possibly be hosts for epigenicic uranium, but so far there is no evidence for this. The Wrangell Mountains on the east and the Alaska Range on the north side of the basin seem to contain better source rocks than the Talkeetna and Chugach Mountains on the west and south, respectively. Further study of these possible source rocks and the depositional history of the basin may suggest the best locations for exploratory driliing.

Some plutonic rocks in the Talkeetna Mountains that were not examineo' by the DGGS party but mapped during 1972 and 1973 by Division personnel may be favorable for U. Felsic intrusives (map unit Jms) of Mesozoic age in the Kings-Kashwitna Rivers area extend in a northeast belt for at least 10 miles (DGGS Annual Report for 1973, p. 14-16). This unit and other granitic and gneissic rocks in the area have not been examined for their uranium potential.

\section{The Chitina River Valley}

The Chitina River valley area is of interest for its $U$ potential because of the presence of probable nonmarine cretaceous sandstones and the rich Kennecott copper mines. Reconnaissance sampling by helicopter was conducted during a 4-day period from a base in the town of chitina. The Chitina River valley is within the Valdez and McCarthy quadrangles and opens into the southeastern part of the Copper River basin (fig. 16). The Mesozoic sediments exposed in the valley provide some information on the subsurface of the Copper River basin, where bedrock is not exposed. Summaries of the geologic setting are included in appendix E under "Copper River basin-Chitina Valley" section and in a DGGS open-file report (Henning, 1973).

Stream-Sediment Sampling

Stream-sediment samples were collected from 105 locations in the Chitina River valley area ( $p l s .6 \& 7)$ :

\begin{tabular}{|c|c|c|c|c|}
\hline & Range & Mean & Threshold & Anomalies \\
\hline Upom (RAA) & $0.30-3.20$ & 1.38 & 2.77 & 5 \\
\hline Uppm (LASL) & $0.46-6.30$ & 2.11 & 3.86 & 4 \\
\hline Th ppm & $0.80-11.80$ & 4.78 & 9.16 & 5 \\
\hline $\mathrm{K}_{2} \mathrm{O} \%$ & $0.31-2.10$ & 1.24 & 1.94 & 2 \\
\hline
\end{tabular}

The $U$ contents of the sediments for the area are low, but the results may reflect subtle anomalies in the bedrock. Two of the stream sediment anomalies (E28 and E29) were found in the northwest part of the McCarthy C-4 quadrangle and one (E14-LASL) was from a tributary to the Kotsina River in the northwest part of the McCarthy $c-4$ quadrangle. Bedrock in the areas of these samples is Triassic Nikolai Greenstone or TriassicJurassic limestone.

Three anomalous $U$ samples (L22, L24, and L28) were collected in granitic rock terrain. $L 22$ and $L 24$ are from the northwest corner of Valdez $B-1$ quadrangle; $L 28$ is fron the south-central part of the Valdez C-2 quadrangle.

Four of the five Th anomalies are from tributaries to Young Creek, an area underlain by Cretaceous sediments. While no $U$ anomalies were 
found in the stream sediments in the area, two water-sample and four of the $f$ ive $k_{2} O$ anomalies were produced. The combined Th and $k_{2} O$ and the $U$ in water anomalies suggest that the cretaceous sediments should be investigated more thoroughly.

Water Sampling

Three of the 74 water samples from the Chitina Valley area were anoma lous:

$\mathrm{H}_{2} \mathrm{O} \mathrm{ppb} \quad \frac{\text { Range }}{0.20-3.50} \quad \frac{\text { Mean }}{0.81} \quad \frac{\text { Threshold }}{2.01} \quad \frac{\text { Anomalies }}{3}$

Two of the anomalous samples were rather closely spaced, on tributaries to Young Creek that drain Cretaceous sedimentary bedrock. The other anomaly was from a tributary to McCarthy Creek that drains from Cretaceous sediments in the McCarthy $8-5$ quadrangle. Considering the generally low values of $U$ found in water samples, these appear to be relatively strong anomalies $(2.10,2.75$, and $3.50 \mathrm{ppb})$.

Bedrock Sampling

Thirty-seven rock samples from the Chitina River valley were analyzed:

\begin{tabular}{|c|c|c|c|c|}
\hline & Range & Mean & Threshold & Anomalies \\
\hline U ppm & $0.40-2.50$ & $\overline{1.23}$ & 2.65 & 0 \\
\hline Th ppm & $0.20-11.30$ & 3.14 & 8.53 & 2 \\
\hline $\mathrm{K}_{2} 0 \%$ & $0.05-3.00$ & 1.55 & 3.18 & 0 \\
\hline
\end{tabular}

The above summary inciudes the results of analyses of both granitic and sedimentary rocks. Two possible anomalous samples, EI6R and EI7R, contained $2.5 \mathrm{ppm} U$. Both of these were from the western part of Maccoll Ridge. Sample El6R is from a small fine-grained felsic intrusive body, and sample El7R is of Cretaceous siltstone.

\section{Aerial Radiometric Survey}

No aerial radiometric survey had been flown by ERDA at the time of the investigation. A single radiometric anomaly was reported by the USGS from a flight up the Chitistone River valley. Bates (1953) recorded an aerial scintillometer reading of $1.0 \mathrm{mr} / \mathrm{hr}$ at one point with a background of only $0.15 \mathrm{mr} / \mathrm{hr}$. This area was not reached by the DGGS party.

\section{Ground Radiometric Survey}

Radiometric responses measured with hand-carried scintilloneters were unimpressive, and generally were below $70 \mathrm{cps}$ on all types of bedrock; counts of 40 to 50 cps were usual. Inspection of the ores at several mines in the Kennecott copper area did not yield any detectable radioactivity. No radiometric survey map was constructed for this region.

The strongest radiometric reading encountered (160 cps) was in the soil bank of a small gully on the east side of a north-flowing tributary to Elliott Creek in the Valdez $\mathrm{C}-1$ quadrangle. This site is on the north- 
west flank of Iron Mountain and is underlain by Triassic limestone and argillite. The spot with the high radioactivity is very small, but is interesting because it is four times the background in the area and is in soil rather than an exposed rock. Stream-sediment samples from near this location were almost anomalous-- -3.40 ppm by Los Alamos' determination. The site seems to warrant some digging to expose the bedrock.

Above-average radiometric readings for the area (90 cps) were found on Maccoll Ridge, over Cretaceous sediments. A value of 100 CDS was recorded for the granite pluton south of the Chitina River in Valdez B-I quadrangle.

\section{Suggestions for Exploration}

The cursory investigation of the Chitina River valley area indicated two areas deserving additional work: the Cretaceous sediments on both sides of Young Creek and the site of a radiometric anomaly on a tributary to Elliott Creek.

The combination of slightly anomalous $\mathrm{Th}$ and $\mathrm{K}_{2} \mathrm{O}$ in sediment samples and $U$ in water samples may be significant in areas of Cretaceous sediments. The writer suggests that the lower parts of the section on the south side of Castle 8 luffs be investigated because these beds are difficult to reach and were not examined.

The importance of the radioactive anomaly on Elliott Creek is unknown; a) though not very strong, it was quite pronounced for that area. Little work would be required to test this site below the soil cover.

The aerial radiometric anomaly reported in the chitistone Canyon by the USGS in 1953 is of unknown significance, but adds to the interest of the region.

\section{Eagle-Charley River Area}

A brief investigation of the Eagle-Circle district in the Yukon River drainage in east-central ( $\mathrm{fig}$. 17) Alaska was conducted by the DGGS during 1975. P.L. Dobey and R.M. Klein spent approximately 1 week collecting samples to aid in determining the potential for oil shale and petroleum in the region. Eighteen of their stream-sediment samples and 16 water samples from a 70-mile-long northwest-trending belt in the northeast corner of the Eagle quadrangle and the southern part of the Charley River quadrangle were submitted for $U$, Th, and $\mathrm{K}_{2} \mathrm{O}$ analyses. These were collected from areas occupied by sedimentary units ranging in age from Devonian to Tertiary (pl. 8). A general description of the geology is included in appendix $E$. Map numbers and general locations are shown in table 4 .

Table 4. Sample locations, Eagle-Charley River area

$\frac{\text { Map No. }}{M I}$

$M 2$

\author{
Location \\ Sediment and water sample, \\ Trib. Coal Creek \\ Sediment and water sample, \\ Sam Creek
}


Table 4. (Cont.)

\begin{tabular}{|c|c|}
\hline Map No. & Location \\
\hline M3 & $\begin{array}{l}\text { Sediment and water sample, } \\
\text { Sam Creek }\end{array}$ \\
\hline$M 4$ & $\begin{array}{l}\text { Sediment and water sample, } \\
\text { Michigan Creek }\end{array}$ \\
\hline M5 & $\begin{array}{l}\text { Sediment and water sample, } \\
\text { mouth of Nation River }\end{array}$ \\
\hline M6 & $\begin{array}{l}\text { Sediment and water samples, } \\
\text { Trout Creek }\end{array}$ \\
\hline$M 7$ & $\begin{array}{l}\text { Sediment and water samples, } \\
\text { Trout Creek }\end{array}$ \\
\hline M8 & $\begin{array}{l}\text { Sediment and water samples, } \\
\text { Trout Creek }\end{array}$ \\
\hline$M 9$ & $\begin{array}{l}\text { Sediment and water sample, } \\
\text { Tutonduk River }\end{array}$ \\
\hline$M 10$ & $\begin{array}{l}\text { Sediment and water sample, } \\
\text { Bryant Creek }\end{array}$ \\
\hline MII & $\begin{array}{l}\text { Sediment and water sample, } \\
\text { Bryant Creek }\end{array}$ \\
\hline 1412 & $\begin{array}{l}\text { Sediment and water sample, } \\
\text { American Creek }\end{array}$ \\
\hline$M 13$ & $\begin{array}{l}\text { Sediment and water sample, } \\
\text { Bluff Creek and Taylor Highway }\end{array}$ \\
\hline$M 14$ & $\begin{array}{l}\text { Sediment and } \mathrm{H}_{2} \mathrm{O} \text { sample, } \\
\text { American Creek }\end{array}$ \\
\hline MIS & $\begin{array}{l}\text { Sediment and } \mathrm{H}_{2} \mathrm{O} \text { sample, } \\
\text { American Creek }\end{array}$ \\
\hline$M 16$ & $\begin{array}{l}\text { Sediment and } \mathrm{H}_{2} \mathrm{O} \text { sample, } \\
\text { American Creek }\end{array}$ \\
\hline MIT & $\begin{array}{l}\text { Water sample, Marion Creek } \\
\text { and Taylor Highway }\end{array}$ \\
\hline$M 18$ & $\begin{array}{l}\text { Sediment and } \mathrm{H}_{2} \mathrm{O} \text { sample, } \\
\text { American Creek campground } \\
\text { and Taylor IIighway }\end{array}$ \\
\hline
\end{tabular}

Siream-Sediment Sampling

A suminary of analyses of 18 stream-sediment samples from the Eagle and Charley River quadrangles is given below:

\begin{tabular}{|c|c|c|c|c|}
\hline & Range & Mean & Threshold & Anomalies \\
\hline Uppm (RAA) & $\overline{0.6-2.50}$ & 1.30 & 2.36 & 1 \\
\hline Th ppm & $1.3-1.7$ & 1.54 & 1.81 & 0 \\
\hline $\mathrm{K}_{2} \mathrm{O} \%$ & $4.0-22.5$ & 10.69 & 22.83 & 0 \\
\hline
\end{tabular}

The one anomalous stream-sediment sample was collected along the Totanouk River in the southeast corner of the Charley River quadrangle. The area is underlain by a series of Precambrian and lower Paleozoic beds. It appears the sample in question was taken near an outcrop of Road River shale. 


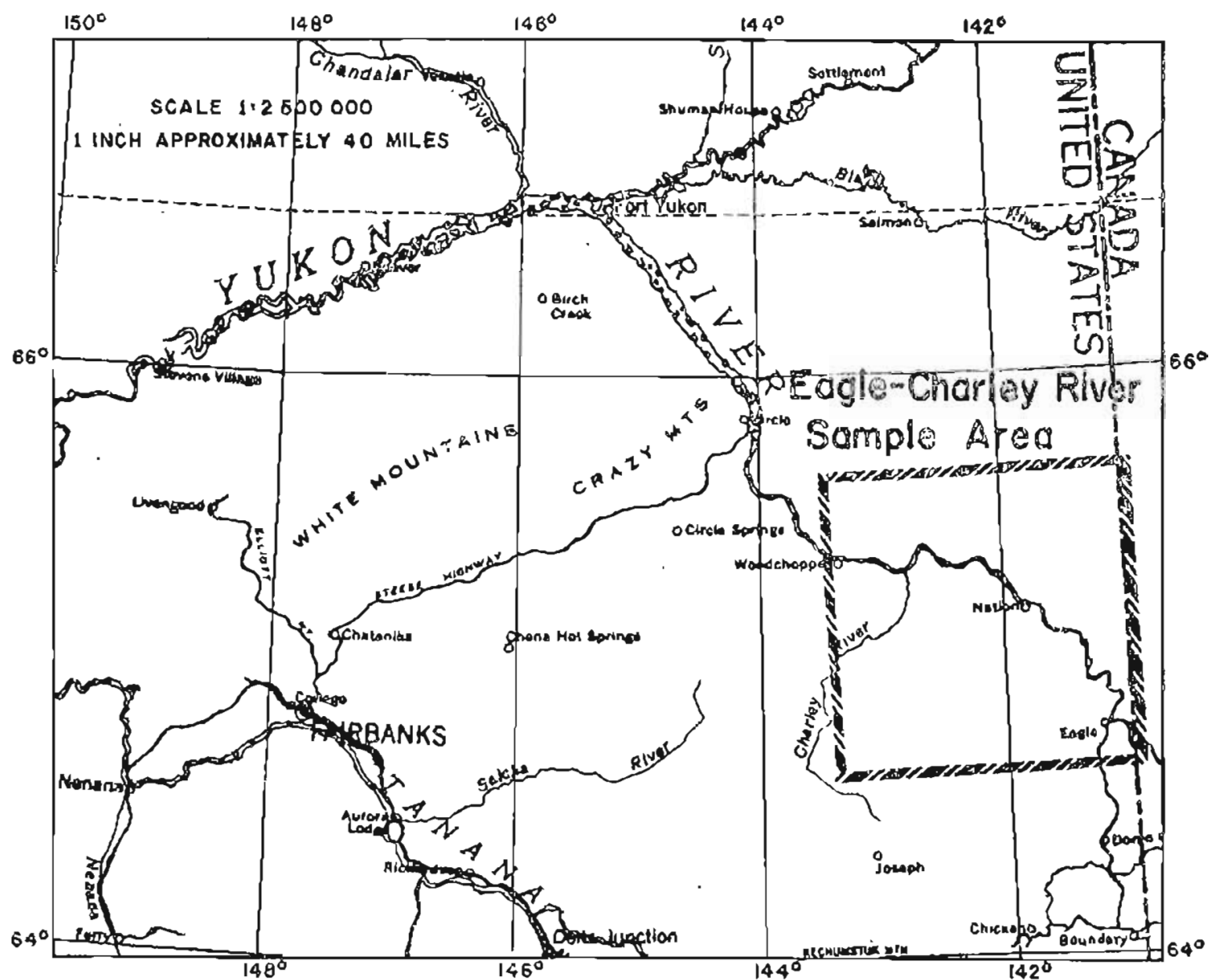

Figure 17. Imlex map of Eagle-Charley River sample area, east-central Alaska.

Water Sampling

Results of analyses of 16 water samples collected at the abovelisted sedinent sample sites are as follows:

$\mathrm{H}_{2} \mathrm{O} \mathrm{ppb}$ $\frac{\text { Range }}{0.1 \cdots 2.2}$

$\frac{\text { Mean }}{0.81}$ $\frac{\text { Threshold }}{2.07}$

Anomalies

The computer analyses produced one anomaly (2.1 ppb) from American creek, just outside the town of Eagle (map M12). This area is predominantly underlain by Tertiary sandstones, tuffs, and siltstones.

Bedrock Sampling

No bedrock samples were submitted for U analyses. 
An aerial radiometric survey was not conducted in this region, but will be flown during 1977, according to a Texas Instruments proposal.

Ground Radiometric Survey

Readings taken with hand-carried scintillometers at the water and sediment sample locations vary from 80 to 400 cps. The three 400-cps readings were at sample locations $M 6, M 7$, and $M 8$, closely spaced sites on Mesozoic Glenn Shale. This unit is predominantly a carbonaceous shale which includes siltstone, quartzite, limestone, and oil shale.

Radiometric readings on a number of scattered outcrops of $G_{i}$ lenn Shale yielded values of $12.0 \mathrm{cps}$. Tertiary shales and sandstones produced between 75 and 165 cps.

\section{Suggestions for Exploration}

The east-central Alaska region has had a limited amount of reconnaissance investigations for uranium. Samples from the Mississippian Ca)ico Bluff formation, however, contain up to 0.02 percent eU (Wedow, White, and Moxham, 1951, p. 106; Wedow, 1954, p. 3, 4), and recent reports (Jonasson and Goodfellow, 1976) describing $U$ discoveries in northern Yukon Territory suggest that the favorable trend may extend into Alaska north of the Yukon River. The writers ( $\rho .72)$ concluded the following environments to be favorable for exploration:

(1) Proterozoic sedimentary and volcanic rocks which are hosts to known occurrences of urunium and copper mineralization.

(2) Ordovician Road River shales which have shown potential as a low-grade high-tonnage sollce of uranium.

(3) Cretaceous (or younger) alkaji granites, syenites, and quartz monzonites which were shown to contain areas of high uranium and fluorine concentrations within a given stock.

(4) Major stratigraphical and structural breaks (i.e. unconformities, faults) betwcen Helikian rocks and younger Proterozoic and paleozoic rocks.

Sediments of Precambrian through Tertiary in age in the region (including the Kandik basin) may have a potential for $U$, and more extensive investigations are certain to follow.

$$
\text { Healy D-I Quadrangie and Vicinity }
$$

A DGGS party headed by W.G. Gilbert conducted a sampling program in

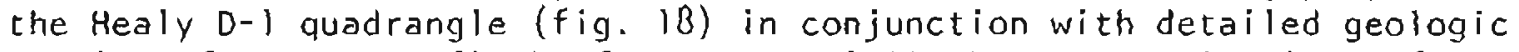
mapping of the north flank of the central Alaska Range. Submitted for $\mathrm{U}$, Th, and $\mathrm{K}_{2} \mathrm{O}$ analyses were 208 stream-sediment samples collected during the 1975 field season. No water or bedrock samples were analyzed for these materials, and neither aerial nor ground radiometric surveys have been conducted over the area. 


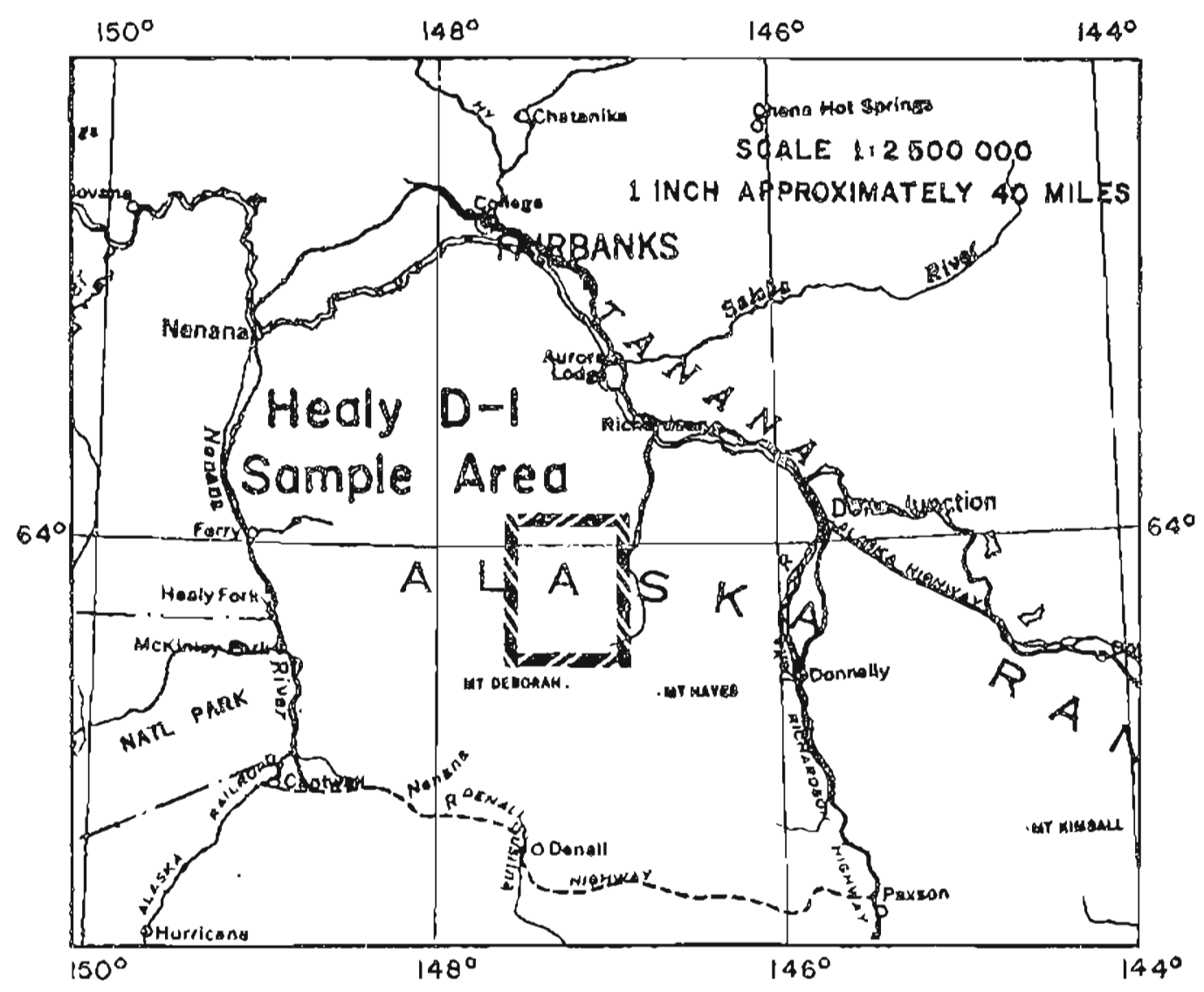

Figure 18. Index map of the Healy D-I sample aren, north flank of the Alaska Range.

The area occupies the foothills and mountains on the north flank of the central part of the Alaska Range, where altitudes in the southern part reach 8,159 feel. Bedrock includes the Precambrian or Early Paleozoic Birch Creek Schist, Keevy Peak Formation, and the Totatlanika Schist. Granitic rocks of Mesozoic age are exposed in the southern half of the D-l quadrangle, and Tertiary roal-Bearing Group sediments are present in the northeastern part. The Tertiary sediments are of interest to uranium prospectors because they display characteristics sinilar to those that are $U$ hosts in Wyoming, and industry explorationists have found $U$ anomalies in stream sediments and water about $50 \mathrm{miles}$ west in this group. The granitic rocks were also considered to warrant study for their $U$ potential. A discussion of the Tertiary Coal-Bearing group is included in appendix $\mathrm{E}$.

Stream-Sediment Sampling

Location of 208 stream-sediment samples from the Healy $D-1$ area appears on plate 9 . Results of the computer analysis are summarized below; analyses were done by Resource Associates of Alaska only; none were submitted to Los Alamos. 


\begin{tabular}{|c|c|c|c|c|}
\hline & Range & Mean & Threshold & Anomal ies \\
\hline Uppm (RAA) & $0.3-10.0$ & 2.13 & 5.40 & 10 \\
\hline Th ppm & $2.0-72.5$ & 19.28 & 40.56 & 8 \\
\hline $\mathrm{K}_{2} 0 \%$ & $1.4-5.2$ & 3.08 & 4.70 & 9 \\
\hline
\end{tabular}

There are a number of anomalies, but none is particularly high. All the $U$ anomalies are over or near ( $2 \mathrm{miles}$ or less) outcrops of granitic rock. Th and $\mathrm{K}_{2} \mathrm{O}$ anomalies are scattered, but $\mathrm{Th}$ appears to be relatively high overall for the rock types in the area.

\section{Suggestions for Exploration}

On the basis of the stream-sediment sampling alone it is difficult to judge the potential for $U$ in the Healy $D-1$ quadrangle. Statistically, there are a number of anomalies, but none is high enough to be especially interescing. It appears the anomalies are associated with the granitic bodies in the southern part of the quadrangle.

The granitic rocks and the Tertiary Coal-Bearing Group, however, should not be condemned, because both have reportedly yielded $U$ anomalies at other locations on the north flank of the Alaska Range and remain underexplored. The Tertiary Coal-Bearing Group is also present up to 200 miles west in the Minchumina basin area, where it is favorably situated structurally and near possible source rocks, but as far as the writer knows, it remains untested for $U$.

\section{CONCLUSIONS}

Uranium exploration in Alaska is still in a preliminary stage. Knowledge of the geology of much of the state is inadequate and the best mechoos to use for uranium exploration in this region demand more research. Field methods to date indicate that stream-sediment sampling and ground radiometric surveys are suitable in areas of bedrock exposures. Water sampling results are erratic, and results of this type of sampling are still being evaluated by ERDA-sponsored programs. Work by the Geological survey of Canada (Jonassen and Goodfellow, 1976) indicates that fluorine shows a close relation to $U$ in stream waters and should be used as an indicator. The aerial radlometric survey of Alaska being conducted on a wide-spaced pattern by ERDA may help define general areas favorable for uranium, or by chance may locate a concentration of radioactive minerals; but because of the wide spacing of flight lines and manner of calculating and reporting anomalies, the results may be misleading and the survey can miss local anomalous areas.

Ceologic studies could include interpreting the tectonic and depositional history of the basinal areas, a study of major unconformicies, and study of 1 ineaments on Landsat (ERTS) images and their relation to $J$ anomalies.

Discoveries of vein-type uranium deposits will probably precede discoveries of sedimentary types, simply because there are considerable areas of exposed alkaline plutonic rocks, and the basins are masked by tundra and water. Most current exploration is directed towards intrusive rock tersains in southeastern Alaska, the Seward Peninsula, the Hogatza plutonic 
belt, and to a lesser extent the central Alaska Range and the YukonKoykuk regions. Late-stage differentiates generally are the most favorable phases for uranium.

Some exploration for sedimentary-type uranium deposits has been conducted in the Tertiary Coal-Bearing Group on the north flank of the Alaska Range, in the Susitna lowland, and in the Tertiary and Cretaceous sediments on the central part of the North Slope. Recent discoveries of uranium associated with copper mineralization in Precambrian sedimentary rocks in northern Yukon Territory, Canada, suggest that similar occurrences could exist in north-central Alaska. Lacking good exposures of sandstones like those in the rimrock country of the Colorado Plateau, evaluation of the uranium potential of the basins in Alaska can be expected to be slow and expensive. Drilling may be the only adequate method, but such tools as track etch, radon-gas sampling, geobotanical prospecting, and geophysics may be useful in some areas. A preliminary project to test the application of radiometric, magnetic, gravimetric, induced polarization, and very low frequency electromagnetic methods to locating roll fronts was conducted in the Selawik lowlands in Alaska during the 1976 field season by J.W. Cady of the USGS and S.W. Hackett of the DGGS. Early results suggest these methods may prove helpful.

The writer considers all the plutons that were examined by the DGGS party in west-central Alaska to be favorable areas for uranium exploration. Rock types, radiometric surveys, and high $U$ and Th contents of numerous stream-sediment and rock samples indicate $U$ and Th enrichment. The headwaters of the Peace River on the northeast flank of Granite Mountain was thought to be one of the most positive target areas for radinactive materials. This area was restaked during the summer of 1976. Alkalic dikes in the Darby Mountains and Zane Hills contain anomalous amounts of $U$ and $T h$ and offer encouragement for exploration. Other localities within the region believed to be more favorable than others are discussed in the text of both parts 1 and 11 . More comprehensive investigations are surely needed.

The Copper River basin and its margins produced little encouragement in the areas that were examined. But the nonmarine sediments in the subsurface, which could not be evaluated because of lack of exposures, may warrant exploratory drilling.

The Chitina River valley did not yield strong anomalies, but the Cretaceous sediments at Maccoll Ridge appear to have characteristics similar to sedimentary uranium host rocks in the western U.S., and a more comprehensive study is suggested. A radioactive anomaly on the northwest flank of Iron Mountain, though very local, is interesting. The source of the radioactivity was not identified.

TBranch of Theoretical and Applied Physics, Denver, CO. 


\section{REFERENCES CITED}

Alaska Division of Geological and Geophysical Surveys annual report for 1973.

Alaska Geological Soclety, 1969-1970a, Copper River stratigraphic correlation section. Tawawe Lake to Moose Creek.

, 1969-1970b, Copper River Basin, Alaska, southwest to northeast

stratigraphic correlation section, Eureka to Rainbow.

, 1969-1970c, Copper River Basin well-location map.

Andreasen, G.E.; Grantz, Arthur; 2ietz, Isidore; and Barnes, D.F., 1964, Geologic interpretation of magnetic and gravity data in the Copper River Basin, Alaska: U.S. Geol. Survey Prof. Paper 316-H, 19 p., maps.

Barnes, F.F., 1951, A review of the geology and coal resources of the Bering River coal field, Alaska: U.S. Cieol. Survey Circ. 146.

Barnes, F.F., Wahrhaftig, Clyde, Kickcox, C.A., Freedman, Jacob, and Hopkins, D.M., 1951, Coal investigations in south-central Alaska, 1944-1946:

U.S. Geol. Survey Bull. 963-E, P. 137-213.

Berg, H.C., and Cobb, E.H., 1967, Metalliferous lode deposits of Alaska: U.S. Geol. Survey Bull. 1246, 254 p.

Borovitskii, V.P., 1976, The application of bog-sampling in prospecting for ore deposits in perennial frost regions: Jour of fieochemical Exploration, May, p. 67-70.

Brabb, E.E., 1965, Stratigraphy and oil possibilities of Mesozoic rocks in Kandik Basin, east-central Alaska (abs.): Am. Assoc. Petroleum beologists, v. 49, no. 10, p. 1757-1758.

Brabb, E.E. and Churkin, Michael, Jr., 1967, Stratigraphic evidence for the Late Devonian age of the Nation River Formation, east-central Alaska: in Geological Survey Research, 1967, chapt. D: U.S. Geol. Survey Prof. Paper 575-D, $291 \rho$.

- 1969, Geologic map of the Charley River quadrangle, east-central Alaska: U.S. Geol, Survey Misc. Geol. Inv. Map 1-573.

Brosge, W.P., and Reiser, H.N., 1962, Preliminary geologic map of the Coleen quadrangle, Alaska: IJ.S. Geol. Survey open-file rept. 370.

Cairnes, D.D., 1914, The Yukon-Alaska international boundary between Porcupine and Yukon Rivers: Canada Geol. Survey Mem. 67, p. 61-65.

Capps, S.R., 1940, Ceology of the Alaska railroad region: U.S. feol. Survey Bull. 907, $196 \mathrm{p}$.

Cass, J.T., 1959, Reconnaissance geologic map of the Nulato quadrangle, Alaska: U.S. Geol. Survey Misc. Creol. Inv. Map 1-291.

Chapin. Theodore, 1918, The Nelchina-Susitna region, Alaska: U.S. Ceol. Survey Bull. 668, 6h $\mathrm{p}$.

Churkin, Michael, Jr., 1973, Paleozoic and Precambrian rocks of Alaska and their role in its structural evolution: U.S. Geol. Survey Prof. Paper 740, $64 \mathrm{p}$.

Cobb, E.H., 1970, Uranium, thorium, and rare-earth elements in Alaska: U.S. Geol. Survey Mineral Inv. Resource Map MR-56.

1972, Metallic mineral resources map of the Candle quadrangle, Alaska: U.5. Geol. Survey Misc. Field Studies Map MF-389.

, 1972, Placer deposits of Alaska: U.S. Geol. Survey open-file rept. 508 .

Dutro, J.T., Jr., and Payne, T.6., 1957, Geologic map of Alaska: U.S. Geol. Survey, scale $1: 2,000,000$. 
Eakin, H.M., 1916, The Yukon-Koyukuk region, Alaska: U.S. Geol. Survey Bull. 631, 85 p.

Eakins, G.R., 1969, Uranium in Alaska:Div. Geol. Survey Geol. Rept. 38, $49 \mathrm{p}$.

, 1975, Iranium investigations in southeastern Alaska: Alaska Div. of Geol. and Geophysical Surveys Geol. Rept. 44, 59 p.

Eakins, G.R., and Forbes, R.B., Investigation of Alaska's uranium potential: U.S. Energy Research and Development Adm. contract AT(05-1)-1627, and Alaska Div. of Geol. and Geophys. Surveys special rept. $12,372 \mathrm{p}$.

Elliott, R.L., and Miller, T.P., 1969, Results of stream-sediment sampling in the western Candle and southern Selawik quadrangles, Alaska: U.S. Geol. Survey open-file report, 61 p., map.

Finch, W.I., 1967, Geology of epigenitic uranium deposits in sandstone in the United States: U.S. Geol. Survey Prof. Paper 538, $97 \mathrm{p}$.

Foster, H.L., 1967, Geology of the Mount Fairplay area, Alaska: U.S. Geol. Survey Bull. 1241-B, P. B1-B18. 1969. Asbestos accurrences in the Eagle C-4 quadrangle, Alaska: U.S. Geol. Survey Circ. 611, $7 \mathrm{p}$. 1972, Preliminary geologic map of the Eagle quadrangle, Alaska: U.S. Geol. Survey Misc. Field Studies Map MF-358.

Foster, H.L., and Keith, T.E.C., 1974, Ultramafic rocks of the Eagle quadrangle, east-central Alaska: U.S. Geol. Survey Jour. Research, V. 2, no. 6, Nov.-Dec., p. 659-669.

Gault, H.R., Kiljeen, P.L., West, W.S., and others, 1953, Reconnaissance of radioactive deposits in the northeastern part of the Seward Peninsula, Alaska, 1945-47 and 1951: U.S. Geol. Survey Circ. 250, 31 p. Grantz, Arthur, 1960a, Geologic map of Talkeetna Mountains (A-1) quadrangle and the south third of Talkeetna Mountains (8-1) quadrangle, Alaska: U.S. Geol. Survey Misc. Geol. Inv. Map 1-314. - 1960b, reologic map of the Talkeetna Mountains A-2 quadrangle, Alaska; and the contiguous area to the north and northwest: U.S. Geol. Survey Misc. Geol. Inv. Map 1-313.

, 1965, Geologic map and cross sections of the Nelchina area, south-central Alaska: U.S. Reol. Survey open-file rept. 255.

Harrington, G.L., 1919, The gold and platinum placers of the KiwalikKoyuk region: U.S. Geol. Survey Bull. 692-G, p. 369-400.

Henning. M.W., 1973, Geologic and mineral evaluation of the Chitina River drainage basin: Alaska Div. Of Geol. and Geophys. Surveys open-file rept. 25, $20 \mathrm{p}$.

Herreid, Gordon, 1965, Geology of the Bear Creek area, Seward Peninsula, Candle quadrangle, Alaska: Alaska Div. Mines and Minerals Geol. Rept. $12,16 \mathrm{p}$.

Imlay, R.W., and Reeside, J.B., 1954, Correlation of Cretaceous formations of Greenland and Alaska: Geol. Soc. America Bull., v. 65, no. 3, p. 223246.

Jonasson, I.R. and Goodfellow; W.D., 1976, Uranium reconnaissance program; Orientation studies in uranium exploration in the Yukon: Geol. Survey of Canada open-file rept. 388 .

Jones, B.K., 1976, Uranium and thorium in granitic and alkaline rocks in western Alaska: Fairbanks, Univ. of Alaska, M.S. thesis, $123 \mathrm{p}$.

Jones, D.L., and Mackevett, E.M., Jr., 1969, Summary of Cretaceous stratigraphy in part of the McCarthy quadrangle, Alaska: U.S. Geol. Survey Bull. 1274-K, 19 p. 
Kimball, A.L:, 1969, Reconnaissance of Tatonduk River red beds: U.S. Bur. Mines open-file report, Il $p$.

Lanphere, M.A., Mackevett, E.M., Jr., and Stern, T.W., 1964, Potassiumargon and lead-alpha ages of plutonic rocks, Bokan Mountain area, Alaska: Science, vol. 145, no. 3633, p. 705-707.

Laudon, L.R., Hartwig, A.E., Morgridge, D.L., and Omernik, J.B., 1966, middle and late Paleozoic stratigraphy, Alaska-Yukon border area between Yukon and Porcupine Rivers: Am. Assoc. Petroleum Geologists Bull., v. 50, no. 9, Sept., o. 1848-1889.

Levorsen, J.A., 1973, Alaska Jexicon reference data: Alaska Div. Cieol. and Geophys. Surveys, 25 p.

Mackevett, E.M., Jr., 1963, Geology and ore deposits of the Bokan Mountain uranium-thorium area, southeastern Alaska: U.S. Geol. Survey Bull. $1154,116 \mathrm{p}$.

, 1971, Stratigraphy and general geology of the McCarthy $\mathrm{C}-5$ quadrangle, Alaska: U.S. Geol. Survey Bull. 1323, 35 p.

Maloney, R.P., and Thomas, B.1., 1966, Investigation of the Purkey-Pile prospects, Kuskokwim River Basin, Alaska: U.S. Bureau of Mines openfile report, $12 \mathrm{p}$.

Martin, G.C., 19!9, The Nenana coal Field, Alaska: U.S. Geol. Survey Bull. $664,54 \mathrm{p}$.

, 1923, A supposed petroleum seepage in the Nenana coal field, in Mineral resources of Alaska, report on progress of investigations

in 1921: U.S. Geol. Survey Bull. 739, p. 137-147.

, 1926, The Mesozoic stratigraphy of Alaska: U.S. Geol. Survey BuाT. 776,493 p.

Matzko, J.J., 1951, Radiometric examination of rock speclmens from Mount Mckinley, Alaska: U.S. Geol. Survey Trace Elements Inv. Rept. 45-C.

Matzko, J.J., and Freeman, V.L., 1963, Summary of reconnaissance for uranium in Alaska, 1955, in Contributions to economic geology of Alaska: U.S. Geol. Survey Bull. 1155, $89 \mathrm{p}$.

Mendenhall, W.C., 1901, A reconnaissance in the Norton Bay region, Alaska (in Reconnaissance in the Cape Nome and Norton Bay regions, Alaska):

U.S. Geol. Survey special publication.

, 1905, Geology of the central Copper River region, Alaska: U.S. Geol. Survey Prof. Paper 41, $125 \mathrm{p}$.

Mertie, J.B., Jr., 1927, Geology of the upper Matanuska Valley, Alaska: U.S. Geol. Survey Bull. 791, $90 \mathrm{p}$.

- 1930, Geology of the Eagle-Circle district, Alaska: U.S. Geol. Survey Bull. 816, 168 p.

, 1932, The Tatonduk-Nation district, Alaska: U.S. Geol. Survey Bull. 836-E, p. 347-444.

, 1937, The Yukon-Tanana region, Alaska: U.S. Geol. Survey Bull. 872, p. 47-48, 51, 54-59, 201-203.

, 1942, Tertiary deposits of the Eagle-Circle district, Alaska:

U.S. Geol. Survey Bull. 917-D, p. 213-262.

Miller, D.J., Payne, T.G., and Gryc, George, 1959, Geology and possible petroleum provinces in Alaska: U.S. Geol. Survey 8ull. 1094, 127 p.

Miller, T.P., 1970, Petrology of the plutonic rocks of west-central Alaska: U.S. Geol. Survey open-flle report, 132 p., maps.

, 1972, Potassium-rich alkaline intrusive rocks of western Alaska: Geol. Soc. America Bull., v. 83, p. 2111-2128, July.

Miller, T.P., and Anderson, L.A., 1969, Airborne radioactivity and totalintensity magnetic survey of the southern kobuk-Selawik lowland, western Alaska: U.S. Geol. Survey open-file rept. 372. 
Miller, T.P., and Bunker, C.M., 1975a, A reconnaissance study of the $U$ and Th contents of plutonic rocks of the south-eastern Seward Peninsula, Alaska: U.S. Geol. Survey open-file rept. 75-217. , 1975b, U, Th, and $K$ analyses of selected plutonic rocks from west-central Alaska: U.S. Geol. Survey open-file rept. 75-2i6.

Miller, T.P., and Elliott, R.L., 1969, Metalliferous deposits near Granite Mountain, eastern Seward Peninsula, Alaska: U.S, Geol. Survey Circ. $614,19 \mathrm{p}$.

Miller, T.P., Elliott, R.L., Finch, W.I., and Brooks, R.A., 1976, Preliminary report on uranium-, thorium-, and rare-earth-bearing rocks near folovin, Alaska: U.S. Geol. Survey open-file rept. 76-710, $13 \mathrm{p}$.

Miller, T.P., and Grybeck, D.G., 1973, Geochemical survey of the eastern Solomon and southeastern Bendeleben quadrangles, Seward Peninsula, Alaska: U.S. Geol. Survey open-file rept. 553, $115 \mathrm{p}$.

Miller, T.P., Grybeck, D.G., Elliott, R.L., and Hudson, T.L., 1972, Preliminary geologic map of the eastern Solomon and southeastern Bendeleben quadrangles, eastern Seward Peninsula, Alaska: U.S. Geol. Survey openfile report, 3 p.

Miller, T.P., and Elliott, R.L., Grybeck, D.G., and Hudson, T.L., 1971, Results of geochemical sampling in the northern Darby Mountains, Seward Peninsula, Alaska: U.S. Geol. Survey open-rile rept. 478.

Miller, T.P., and Ferrians, 0.J., Jr., 1968, Suggested areas for prospecting in the central Koyukuk River region, Alaska: U.S. Geol. Survey Circ. $570,12 . P$.

Miller, T.P., Patton, W.W., Jr., and Lanphere, M.A., 1966, Preliminary report on a plutonic belt in west-central Alaska, in Geological Survey research-1966: U.S. Geol. Survey Prof. Paper 550-D, p. D 158-0162.

Moffit, F.H., 1905, The Fairhaven gold placers, Seward Peninsula, Alaska: U.S. Geol. Survey Bull. 247, 85 p., maps.

1938, Geology of the Chitina Valley and adjacent area, Alaska: U.S. Geol. Survey Bull. 894, $131 \mathrm{p}$.

Moxham, R.M., and Nelson, A.E., 1952, Reconnaissance for radioactive deposits in south-central Alaska, 1947-49: U.S. Geol. Survey Circ. 184, p. 11-14.

Nininger, R.D., 1954, Minerals for otomic energy: 0 . Van Nostrand Co., Inc., $347 \mathrm{p}$.

Paige, Sidney, and Knopf, Adolph, 1907, Cienlogic reconnaissance in the Matanuska and Talkeetna basins, Alaska: U.S. Geol. Survey Bull. 327, $68 \mathrm{p}$.

Patton, W.W., Jr., 1967, Regional geologic map of the Candle quadrangle, Alaska: U.S. Geol. Survey Misc., Geol. Inv. Map I-492.

1970, Petroleum possibilities of the Yukon-Koyukuk province, Alaska: U.S. Geol. Survey open-file report, 13 p., map.

, 1973, Reconnaissance geology of the northern Yukon-Koyukuk province, Alaska: U.S. Geol. Survey Prof. Paper 774-A, $17 \mathrm{p}$.

Patton, W.W., Jr., and Hoare, J.M., 1968, The Kaltag fault, west-central Alaska: U.S. Geol. Survey Prof. Paper 600-0, p. D147-D153.

Patton, W.W., Jr., and Miller, T.P., 1966, Regional geologic map of the Hughes quadrangle, Alaska: U.S. Geol. Survey Misc. Geol. Inv. Map 1-459.

- 1968, Regional geologic map of the Selawik and southeastern Baird Mountains quadrangles, Alaska: U.S. Geol. Survey Misc. Geol. Inv. Map $1-530$.

Patton, W.W., Jr., Miller, T.P., and Tailleur, I.L., 1968, Regional geologic map of the Shungnak and southern part of the Ambler River quadrangles, Alaska: U.S. Geol. Survey Misic. Geol. Inv. Map 1-554. 
Payne, T.G., 1955, Mesozoic and Cenozoic tectonic elements of Alaska: U.S. Geol. Survey Misc. Geol. Inv. Map 1-84.

Péwe, T.L., 1975, Quaternary geology of Alaska: U.S. Geol. Survey prof. Paper $835,122 \mathrm{p}$.

Reed, 3.C., 1961, Geology of the Mount Mckinley quadrangle, Alaska: U.S. Geol. Survey 8 ul?. 1108-A, 36 p., map.

Reed, B.L., and Lanphere, M.A., 1974, Offset plutons and history of movement along the Mckinley segment of the Denali Fault system, Alaska: Geol. Soc. America Bull. v. 85, p. 1883-1892, Dec.

Runnelis, D.D., 1964, The copper deposits of Ruby Creek, Cosmos Hills, Alaska: Cambridge, Mass., Harvard University, Ph.D. thesis, May 1963.

Sainsbury, C.L., 1974, Geologic map of the Bendeleben 1:250,000 quadrangle, Seward Peninsula, Alaska: The Mapmakers, P.0. Bax 145. Anchorage, Alaska, $37 \mathrm{p}$. and map.

Sainsbury, C.L., Hedge, C.E., and Bunker, C.M., 1970, Structure, stratigraphy, and isotopic composition of racks of Seward Peninsula, Alaska: Am. Assoc. Petroleum rieologists Bull., v. 54, no. 12, p. 2502-2503, Dec.

Saunders, R.H., 1952, Fred J. Jenkins property, Eagle, Alaska: Terr. of Alaska Dept. Mines and Minerals Prospect Examination 60-3 (unpub.). , 1955, Berg copper prospect, Alaska Dept. of Mines Prospect Examination 28-2 (unpub.). , 1956, Berg copper prospect: Alaska Dept. of Mines Prospect Examination 28-3 (unpub.). , 1962, Bear Creek prospect: Alaska Div. Of Mines and Minerals Prospect Examination 28-4 (unpub.).

Schrader, F.C., 1904, A reconnaissance in northern Alaska: U.S. Geol. Survey Prof. Paper 20, $139 \mathrm{p}$.

Smith, J.f., and Mackevett. E.M., Jr., 1970, The Skolai group in the McCarthy $B-4, C-4$, and $C-5$ quadrangles, Wrangell Mountains, Alaska: U.S. Geol. Survey Bull. 1274-0, 26 p.

Sinith, P.S., 1913, The Noatak-Kobuk region, Alaska: U.S. Reol. Survey Bull. $536,160 \mathrm{p}$.

, 1939, Aerial geology of Alaska: U.S. Geol. Survey Prof. Paper 192,92 p., maps.

Smith, P.S., and Eakin, H.M., 1911, A geologic reconnaissance in southwestern Seward Peninsula and the Nortan Bay-Nulato region, Alașka: U.S. Geol. Survey Bull. 449, $142 \mathrm{p}$.

Stephens, F.H., 1971, the Kendrick Bay project: Western Miner, October, p. $151-158$.

Taucher, L.M., 1971, Uranium exploration in southwest Texas, in Selected papers from 1970 uranium symposium at Socorro, New Mexico: New Mexico Bur. of Mines and Mineral Resources Circ. $118,61 \mathrm{p}$.

Texas Instruments, Inc., 1975, Airborne geophysical survey, Copper River and Seward-Selawik areas, Alaska: Prepared for U.S. Energy Research and Development Adm., contract AT(05-1)-1653.

Triplehorn, D.M., 1974, Clay mineralogy and petrology of the Coal-Bearing Group near Healy, Alaska: Alaska Div. of Geol. and heophys. Surveys Geol. rept. $52,15 \mathrm{p}$.

U.S. Reological Survey, 1962, reological Survey Research, 1962: rieol. Prof. Paper 450-A.

, in cooperation with Alaska Department of Natural Resources, 1964, Mineral and Water Resources of Alaska: U.S. Government Printing Office, 177 p., maps. 
, 1967, Geological survey research, 1967: U.S. Geol. Survey Prof. Paper 575-A.

Wahrhaftig, Clyde, 1944, Coal deposits of the Costello Creek Basin, Alaska: U.S. Geol. Survey open-file rept. 8.

- 1958, Quaternary and engineering geology in the central part of the Alaska Range: U.S. Geol. Survey Prof. Paper 293, $115 \mathrm{p}$.

- 1970a, Geologic map of the Healy D-2 quadrangle, Alaska: U.S.

Geol. Survey Geol, Quad, Map G0-804.

, 1970b, Geologic nap of the Healy D-3 quadrangle, Alaska: U.S.

Geol. Survey Geol. Quad. Map G0-805.

, 1970c, Geologic map of the Healy D-4 quadrangle, Alaska: U.S.

Geol. Survey Geol. Quad. Map GQ-806.

, 1970d, Geologic map of the Healy D-5 quadrangle, Alaska: U.S.

Geal. Survey Geol. Quad. Map GQ-807.

, 1970e, Geologic map of the Fairbanks A-2 quadrangle, Alaska:

U.S. Geol. Survey Quad. Map 6Q-808.

- 1970f, Geologic map of the Fairbanks A-3 quadrangle, Alaska:

U.S. Geol. Survey quad. Map 60 -809.

, 1970g, Geologic map of the Fairbanks A-4 quadrangle, Alaska:

U.S. Geol. Survey Quad. Map GQ-810.

, 1970h, Geologic map of the Fairbanks A-5 quadrangle, Alaska:

U.S. Geol. Survey Quad. Map G0-811.

Wahrhaftig, Clyde, and Hickcox, C.A., 1955, Geology and coal deposits, Jarvis Creek coal Field, Alaska: U.S. Geol. Survey Bull. 989-G, p. 353366.

Wahrhaftig. Clyde, Wolfe, J.A., Leopold, E.B., and Lanphere, M.A., 1969, The Coal-Bearing Group in the Nenana coal field, Alaska: U.S. Geol. Survey Bull. 1274-0, $29 \mathrm{p}$.

Weber, F.R., 1971, Preliminary engineering geologic map of proposed transAlaska pipeline route, Mt. Hayes quadrangle: U.S. Geol. Survey openfile report $493(R-8)$.

Wedow, Helmuth, Jr.. 1954a, Reconnaissance for radioactive deposits in the Eagle-Nation area, east-central Alaska, 1948: U.S. Geol. Survey Circ. B16. 9 p.

- 1956, Summary of reconnaissance for radioactive deposits in Alaska, 1945-I954, and an appraisal of Alaskan uranium possibilities: U.S. Geol. Survey Trace Elements Inv. Rept. 577, $113 \rho$.

Wedow, Helmuth, Jr., Killeen, P.L. and others, 1954, Reconnaissance for radioactive deposits in eastern interior Alaska, 1946: U.S. Feol. Survey Circ. 331 , p. 16-18.

Wedow, Helmuth, Jr., White, M.G., and others, 1954b, Reconnaissance for radioactive deposits in east-central Alaska, 1949: U.S. Geol. Survey Circ. 335, $22 \mathrm{p}$.

Wedephol, K.H., 1969, Handbook of Geochemistry: published in Berlin, Germany.

West, W.S., 1953, Reconnaissance for radioactive deposits in the Darby Mountains, Seward Peninsula, Alaska, 1948: U.S. Geol. Survey Circ. 300,7 p., map.

West, W.S. and Matzko, J.J., 1953, Buckland-Kiwalik district, 1947, in Reconnaissance for radioactive deposits in the northeastern part of the Seward Peninsula, Alaska, 1945-47 and 1951: U.S. Geol. Survey Circ. 250,31 p.

White, M.G., 1950, Examination for radioactivity in a copper-lode prospect on Ruby Creek, Kobuk River Valley, Alaska: U.S. Geol. Survey Trace Elements Inv. Rept. 76-A, 8 p., maps. 
, 1952, Radioactivity of selected rocks and placer concentrates from northeastern Alaska: U.S. Geol. Survey Circ. 135, $12 \mathrm{p.}$

, 1952, Reconnaissance for radioactive deposits along the upper Porcupine and lower Coleen Rivers, northeastern Alaska: U.S. Geol. Survey Circ. 185,13 p., 3 figs.

White, M.G., and Tolbert, G.E., 1954, Miller House-Circle Hot Springs area, in Reconnaissance for radioactive deposits in east-central Alaska, 1949: U.S. Geo\}. Survey Circ. 335, 22 p.

Williams, J.R., 1970, Ground water in the permafrost regions of Alaska: U.5. Geol. Survey Prof. Paper 696, $76 \mathrm{p}$. 
Appendix A

STREAM-SEDIMENT-, ROCK-, ANO WATER-SAMPLE ANALYSES WITH RADIOMETRIC DATA

Sediment , rock , and water-sample data collected and analyzed were categorized into seven groups according to geologic and geographic setting. The groups are: (1) Granite Mountain area (Candle quadrangle), (2) the Darby Mountains (Solomon and Bendeleben quadrangles) and the Selawik Hills area (Selawik quadrangle), (3) the Zane Hills and Purcell Mountains area (Hughes and Shungnak quadrangles), (4) the Copper River basin area (Talkeetna Mountains, Mount Hayes, and Gulkana quadrangles), (5) the Chitina River valley area the (McCarthy and Valdez quadrangles), (6) the Eagle-Charley River area (Eagle and Charley River quadrangles), and (7) the Healy area (Healy quadrangle).

Means, standard deviations, and threshold values of concentrations of uranium, thorium, and potassium oxide were calculated for samples in each of the above-mentioned areas. Threshold values, above which sample concentrations are considered to be anomalously high and therefore of special interest, are concentrations two standard deviations above the sample mean. Samples containing concentrations considered anomalous have been underlined in the following table.

Correlation coefficients were calculated to see if there was a relationship between the stream-sediment analyses for uranium by Resource Associates, sediment analyses for the uranium by Los Alamos Scientific Laboratories, and the uranium in water samples. The histograms included in appendix B plot the concentrations of uranium in these three categories.

Correlation coefficients were also calculated to discern any relationship between the uranium concentrations reported by Resource Associates and the thorium and potassium oxide concentrations reported by the DGGS laboratory. 
Table A-l. Stream-sediment, bedrock, and water analyses and radiometric data. Concentrations considered anomalous are underlined; those near anomalous are dashed.

Granite Mountain Area

Sediment and Water

\begin{tabular}{|c|c|c|c|c|c|c|c|c|}
\hline $\begin{array}{l}\text { Map } \\
\text { No. } \\
\text { E I }\end{array}$ & $\begin{array}{l}\text { Field No. } \\
\text { Gi:l } 6865\end{array}$ & $\begin{array}{l}\text { U PPM } \\
\text { (RAA) } \\
\theta .40\end{array}$ & $\begin{array}{l}\text { Th ppm } \\
1.3 .50\end{array}$ & $\begin{array}{l}U / \text { Th } \\
0.62\end{array}$ & $\begin{array}{l}\% \mathrm{~K}_{2} \mathrm{O} \\
0.0 \% 2\end{array}$ & $\begin{array}{l}U p p m \\
(L A S L)^{2}\end{array}$ & $\begin{array}{l}\mathrm{H}_{2} \mathrm{O} \text { ppb } \\
(\mathrm{LASL})^{2}\end{array}$ & cps \\
\hline $\mathrm{B} 2$ & $5 E \perp 645$ & 5.80 & $1.5,40$ & 0.38 & 1.20 & 0.1 .4 & 0.10 & 50 \\
\hline$\sqrt{13}$ & 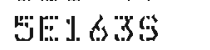 & 3.60 & 13.50 & 0.27 & 0.52 & 3.60 & $0.2 E$ & 50 \\
\hline$B A$ & $5 E, 6 \% 5$ & 2.70 & $2 x .20$ & 0.12 & 1.58 & 7.90 & 0.85 & \\
\hline 85 & $51: 186$ & A. 40$)$ & 10.70 & $0.4 \%$ & 1.35 & 5.80 & 0.35 & A \\
\hline$B 6$ & $5 E .629$ & $\therefore 10$ & 11.00 & 0.1 .9 & $1.2=$ & 2.30 & 0.25 & \\
\hline 117 & $5 F 36 \%$ & 1. .50 & 3.65 & 0.41 & 0.98 & 3.30 & 0.40 & 50 \\
\hline$B B$ & GE:LS\$S & 2.10 & 17.70 & $0.1 . A$ & 1.84 & 8.50 & 0.35 & 90 \\
\hline (B) & SE」ひOS & 2.70 & 1.6 .70 & 0.16 & $1.9 \%$ & 6.00 & 1. .25 & 30 \\
\hline$\dddot{810}$ & SEปSBS & 2.00 & 32.830 & 0.06 & 1.80 & 1.7 .90 & 0.00 & 100 \\
\hline$x 11$ & 沾ELラフタ & 3.70 & 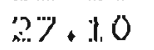 & 0.1 .4 & 1.70 & 1.0 .40 & 0.15 & 100 \\
\hline$H 12$ & $5 E 1565$ & $6 \cdot 20$ & 29.20 & 0.21 & 1.72 & $1.3 \cdot 30$ & 0.30 & 1.00 \\
\hline [3. 3 & 58: & $6 \cdot 20$ & 1.6 .40 & 0.38 & 1.69 & 10.40 & $0.0 \%$ & 100 \\
\hline 114 & $50+969$ & 7.10 & 18.30 & 0.39 & 1.39 & 9.40 & 0.35 & 1.00 \\
\hline 415 & 501978 & 9.20 & 30.00 & 0.31 & 1.82 & 1.7 .70 & 1.1 .0 & 1.00 \\
\hline a. 6 & $\because ; 0 ; 1949$ & 6.60 & 1.1 .50 & $0.5 \%$ & 1.33 & 9.70 & & 100 \\
\hline 917 & (3). & 4.60 & 10.00 & $0.4 B$ & 1.68 & 7.60 & 1.00 & 100 \\
\hline$[1.8$ & $50 \times 918$ & $1.1 .70)$ & J. & 0.75 & 1.25 & 12.80 & 0.10 & 90 \\
\hline$\forall \perp 9$ & 50,925 & 6.90 & 19.50 & 0.35 & 1.72 & 10.00 & 0.45 & 10 \\
\hline 120 & 501908 & 10.90 & $2 \div 4.00$ & 0.45 & 2.15 & $35 \cdot 10$ & & 100 \\
\hline 121 & 501898 & $3 \cdot 6(0)$ & 10.50 & 0.34 & $1 \cdot 61$ & 9.00 & 0.79 & 100 \\
\hline a? & 501886 & 6.10 & 20.00 & 0.31 & 246 & 10.60 & 0.60 & 100 \\
\hline 623 & $5018 \% 8$ & 8.80 & 17.50 & 0.50 & 2.33 & $1.7 \cdot 1.0$ & 1.30 & 100 \\
\hline 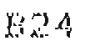 & 810775 & 0 & 3.80 & 1.47 & 2.62 & $12 \cdot 10$ & 0.50 & 250 \\
\hline 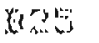 & 50729 & $\therefore .10$ & 11.20 & $0.2 \%$ & .1. $4 \mathrm{l}$ & & 2.15 & 60 \\
\hline 326 & SE73 & 0.70 & 2.50 & 0.28 & 0.62 & 7.10 & & 20 \\
\hline 1827 & $5 E 769$ & $0+$ & 3.06 & 0. & $0.0 \$$ & 1.40 & 1.50 & 20 \\
\hline 528 & $5 E 7 A S$ & 0.30 & 2.24 & 0.13 & 0.79 & 1.60 & 1.00 & ?o \\
\hline $82 \%$ & $5 E 75$ & .1. .0 & 0.00 & & 0.82 & 1.90 & 1. . 45 & 20 \\
\hline 1330 & 50686 & 18.80 & 8.25 & 2.28 & .7. & 4.30 & 0.45 & 100 \\
\hline B3.31. & $5 c 67 s$ & 2.90 & 37.50 & 0.08 & 2.46 & 35.00 & 1.65 & 250 \\
\hline 832 & 50695 & 2.10 & 15.50 & 0.14 & 1. . & 1.00 & & d.50 \\
\hline E.33 & $5 E 728$ & 1.70 & $2+150$ & 0.68 & $\mathrm{~L} .0 \mathrm{~L}$ & 4.10 & (). & \\
\hline 1834 & $5 E 715$ & 10.20 & 32.70 & 0.31 & 1.98 & 24.00 & 0.65 & 200 \\
\hline$B 135$ & SE: & 10.80 & 7.50 & 1.44 & 1.43 & 20.00 & 0.95 & 250 \\
\hline 1.36 & 5E69s & 11.30 & $5 \Omega \cdot 50$ & 0.2 .2 & 1.73 & 05.00 & $1 \cdot .0)$ & 300 \\
\hline 1837 & "تË685 & 0.80 & 50 & 0,16 & 3.34 & 8.10 & 0.50 & 3.50 \\
\hline 838 & SEGAS & 7.90 & 34.30 & 0.33 & 3.07 & 1.4 .70 & 0.75 & 200 \\
\hline 189 & $51=639$ & 2.40 & 37.60 & 0.06 & 2.36 & 14.70 & 1. . $0 \mathrm{~g}$ & 1.00 \\
\hline BAO & 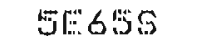 & 4.90 & 25,50 & 0.19 & 3.01 & 3.70 & 0.85 & \\
\hline$B A 1$ & 51:665 & 5.20 & 50.50 & 0.10 & 2.97 & 10.80 & 0.5 & 250 \\
\hline 442 & 5Cs & 4.90 & 13.60 & 0.36 & 2.29 & 6.80 & 0.30 & \\
\hline$x \rightarrow 43$ & $5 \mathrm{c} 615$ & 4.50 & 16.80 & 0.27 & 1.93 & 6.30 & 0.56 & \\
\hline BAA & 50629 & 4.30 & 21.30 & 0.20 & 2.37 & 10.10 & 1.913 & 40) \\
\hline 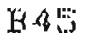 & 55613 & 9.60 & 21.00 & 0.16 & 2.01 & 14.50 & 0.30 & \\
\hline PQMG & FE609 & 7.60 & 16.50 & 0.46 & $\therefore .08$ & 1.4 .60 & 1.20 & \\
\hline
\end{tabular}

IAnalyses performed by Resource Associates of Alaska, Inc.

2 Analyses performed by Los Alamos Scientific Laboratory. 


\begin{tabular}{|c|c|c|c|c|c|c|c|c|}
\hline $\begin{array}{l}\text { Map } \\
\text { No. } \\
\text { li. } 97\end{array}$ & $\begin{array}{l}\text { Field No. } \\
\text { wi: } 599\end{array}$ & $\begin{array}{l}\text { U ppm } \\
\text { (RAA) } \\
1.1 .90\end{array}$ & $\begin{array}{l}\text { Th ppm } \\
2: 30\end{array}$ & $\begin{array}{l}\text { U/Th } \\
0.5 .5\end{array}$ & $\begin{array}{l}\% \mathrm{~K}_{2} \mathrm{O} \\
2.8 \mathrm{I}\end{array}$ & $\begin{array}{l}\text { U ppm } \\
(\text { LASL) } 1 \\
1.8 .40\end{array}$ & $\begin{array}{l}\mathrm{H}_{2} \mathrm{O}_{\mathrm{ppb}} \\
(\mathrm{LASL}){ }^{1} \\
1.1 \mathrm{i}\end{array}$ & $\begin{array}{l}\operatorname{cps} \\
\text {.1. } 40\end{array}$ \\
\hline 848 & 51589 & $12+60$ & $26 \cdot 30$ & 0.48 & 2.86 & 1.8 .00 & $0+100$ & 140 \\
\hline 1849 & $30: 89$ & 6.60 & 28.50 & 0.08 & 2.63 & 1.4 .90 & 1.25 & 年 \\
\hline 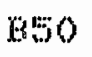 & :059s & 9.20 & $47+50$ & 0.1 .9 & 2.63 & 21.60 & 1.30 & 200 \\
\hline MBS\% & BEs & 20.30 & 19.30 & 1.05 & 1.94 & & & \\
\hline 802 & $5 E 428$ & 1. 20 & 3.00 & 0.40 & 3.03 & 1.60 & 1.00 & 40 \\
\hline 1853 & 30398 & $2 ?+40$ & $16 \cdot 60$ & 0.1 .4 & 1.05 & 2.30 & 0.60 & 40 \\
\hline$B: 34$ & 50405 & 200 & $7.7 \%$ & 0.34 & 1.09 & 4.00 & 0.130 & 40 \\
\hline B: & FEA 1.9 & 3.10 & $6+90$ & 0.45 & $\therefore 40$ & 8.90 & 0.60 & 40 \\
\hline 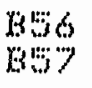 & $\begin{array}{r}5385 \\
60378\end{array}$ & $\begin{array}{l}3.00 \\
1.50\end{array}$ & $\begin{array}{l}9.57 \\
3.30\end{array}$ & $\begin{array}{l}0.31 \\
0.45\end{array}$ & $\begin{array}{l}1.29 \\
1.00\end{array}$ & $\begin{array}{l}3.60 \\
2.40\end{array}$ & $\begin{array}{l}0.360 \\
0.360\end{array}$ & $\begin{array}{l}50 \\
60\end{array}$ \\
\hline 868 & : 409 & 2.70 & 7.60 & 0.36 & 1. .34 & 4.90 & 1.00 & \\
\hline 259 & 50.366 & 8.50 & 24.00 & 0.35 & 1.72 & 13.50 & 0.313 & $2 !: 00$ \\
\hline 860 & 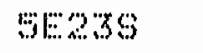 & 6.60 & $34+80$ & 0.16 & 2.74 & 10.70 & 0.70 & \\
\hline 1861 & 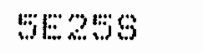 & 24.40 & 23.60 & 0.33 & 4.1.14 & $3: 3.00$ & $1.0 \%$ & 200 \\
\hline 1362 & : : & 21.20 & 28.60 & $0.2 \%$ & 3.72 & 49.00 & $1+70$ & 200 \\
\hline 863 & :1:200 & 10.70 & $68+170$ & 0.16 & 3.89 & 29.00 & 1.10 & $2 \%$ \\
\hline 864 & 60.318 & 9.00 & 20.00 & 0.45 & 2.14 & 1.4 .30 & 1.35 & 180 \\
\hline 868 & 1006 & 9.60 & $2 \% .00$ & 0.36 & 2.00 & $18+10$ & $1.6 \%$ & 130 \\
\hline 1366 & (3) & 9.70 & 12.20 & 0.80 & $1 .+83$ & 1.90 & 1.30 & 1.40 \\
\hline $86 \%$ & 30236 & 9.40 & I. 1.40 & 0.82 & 1.73 & & & 170 \\
\hline 1868 & 60226 & 7.1 .0 & 3.3 .00 & $0+2 \%$ & 1.78 & & 0.70 & 1.00 \\
\hline 869 & 60218 & 7.20 & 13.90 & 0.152 & 2.00 & 1.1 .50 & 1.00 & 100 \\
\hline 1870 & 30169 & 10.60 & 24.90 & 0.42 & 2.75 & $16 \cdot 60$ & 1.00 & 200 \\
\hline 371 & 50178 & 28.00 & 22.30 & $1+26$ & $2.1 \%$ & 44.00 & 0.95 & 1.70 \\
\hline 172 & 50209 & 7.30 & 20.00 & 0.37 & 2.60 & I. L . 20 & 1.83 & 100 \\
\hline 573 & $51: 215$ & 2.40 & 16.30 & 0.115 & 1.66 & & & 1.50 \\
\hline 174 & $51: 220$ & 7.60 & 360 & 0.22 & $4.6 \%$ & 39.00 & 0.45 & 100 \\
\hline 1373 & 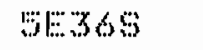 & $6+100$ & 37.40 & 0.1 .7 & 1.03 & 1.7 .40 & 0.45 & 273 \\
\hline 1876 & $31: 376$ & 7.40 & 26.30 & 0.28 & 1. .3. & $14+00$ & 1.30 & 250 \\
\hline 877 & 513335 & 8.60 & $4 \% .00$ & $0+18$ & 2.76 & 22.00 & 0.100 & 200 \\
\hline 878 & $5: 1: 345$ & 6.20 & 36.00 & 0.1 .7 & $2+40$ & 12.90 & 1.24 & \\
\hline 1379 & $80-385$ & 3.80 & 27.30 & 0.1 .4 & 2.08 & $113+30$ & 1.30 & 120 \\
\hline 1380 & 60326 & 3.70 & 22.30 & 0.1 .7 & $1.8 \%$ & 9.20 & 0.85 & 1.90 \\
\hline $58 \%$ & 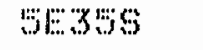 & $5+20$ & 17.80 & 0.29 & 2.00 & 1.0 .30 & 0.90 & \\
\hline 882 & 35439 & 3.30 & 23.00 & 0.1 .4 & 1.76 & 10.80 & 0.80 & 80 \\
\hline 583 & 50428 & $6+20$ & 33.80 & $0+18$ & 2.22 & 1.4 .90 & $0 .: 30$ & 100 \\
\hline 584 & 50415 & 8.70 & 1.3 .100 & 0.64 & 2.04 & 6.40 & 0.40 & 1.00 \\
\hline 1085 & 50465 & 2.90 & 14.80 & 0.20 & $1.9 \%$ & 5.40 & 1.35 & 30 \\
\hline 1886 & 50498 & 2.30 & 4.00 & 0.988 & 1.49 & 3.50 & 0.50 & 60 \\
\hline 887 & 50489 & 3.20 & 10.00 & 0.32 & 1.89 & 3.90 & 0.80 & 60 \\
\hline 588 & 5008 & $\because 1.0$ & 7.00 & 0.30 & 1. . 72 & & 0.40 & 80 \\
\hline 1889 & 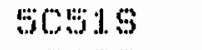 & $2+70$ & 11.00 & 0.25 & $1.7 \%$ & 1.0 .70 & 0.40 & 70 \\
\hline 1890 & $5 E 49 S$ & 2.00 & 1.1 .60 & 0.17 & 1.78 & 3.30 & 0.35 & 1.100 \\
\hline $19 \%$ & 50528 & 1.70 & 8.00 & 0.21 & 1.74 & & & 60 \\
\hline 1892 & 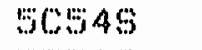 & 2.70 & 10.50 & 0.26 & 1.070 & 13.20 & $\mathrm{~J}, 09$ & 90 \\
\hline 1893 & $6 E 515$ & 2.00 & 9.00 & 0.22 & 1.26 & 3.20 & 0.20 & \\
\hline 1394 & 60635 & 1.90 & 34.60 & 0.06 & 1.30 & & & 60 \\
\hline 195 & : & 2.00 & 9.20 & 0.22 & 1.49 & & 0.80 & 160 \\
\hline 896 & 30 & 2.70 & 11.00 & 0.23 & 1.02 & & & 40 \\
\hline 1897 & 6066 & 2.90 & $\ddot{3}+7 \%$ & 0.50 & 1. 48 & 2.40 & $1 .+1 .: 3$ & 40 \\
\hline 1998 & $31: 195$ & 1.00 & 10.40 & 0.1 .0 & 1.62 & & $0.6 \%$ & 60 \\
\hline
\end{tabular}

1. See p. A-2. 


\begin{tabular}{|c|c|c|c|c|c|c|c|c|}
\hline $\begin{array}{l}\text { Map } \\
\text { No. } \\
\text { Hig9 }\end{array}$ & $\begin{array}{l}\text { Field No. } \\
5135\end{array}$ & $\begin{array}{l}\text { U ppm } \\
(\text { RAA) } \\
3.30\end{array}$ & $\begin{array}{l}\text { Th } \mathrm{ppm} \\
1.0 .40\end{array}$ & $\begin{array}{l}U / T h \\
0.32\end{array}$ & $\begin{array}{l}\% \mathrm{~K}_{2} \mathrm{O} \\
1.84\end{array}$ & $\begin{array}{l}U \text { ppm } \\
(\text { LASL) } 1 \\
4.00\end{array}$ & $\begin{array}{l}\mathrm{H}_{20} \mathrm{ppp} \\
(\mathrm{LASL}) \\
0+30\end{array}$ & cps \\
\hline$x 100$ & 60435 & 1.20 & 3.60 & 0.34 & $0.8 \%$ & 1.60 & $0.9 \%$ & 24 \\
\hline B. & 60458 & 1.90 & 3.00 & 0.6 .3 & $0.8 \%$ & 1.40 & 0.60 & 24 \\
\hline B.102 & 50445 & 1.70 & 4.50 & 0.38 & 0.86 & 1.60 & 0.30 & 24 \\
\hline H103 & $50,4 \% 5$ & 3.30 & 20.00 & 0.1 .7 & 2.06 & & & 40 \\
\hline 81.04 & tives & 1.70 & 13,40 & 0.13 & 1.1 .3 & & $1+13$ & 40 \\
\hline$B 10 \%$ & :3E:318 & & & & 2.41 & & 0.75 & 200 \\
\hline 8106 & 50309 & 6.70 & 3.3 .50 & 0.20 & $2.4 \%$ & 19.70 & 0.40 & 250 \\
\hline B.10\% & GE:328 & 7.90 & $\because 0.00$ & 0.16 & 2.46 & $1 \% .60$ & 0.45 & 3 \\
\hline 8108 & 50165 & 16.80 & $16+80$ & 1.00 & 2.230 & 24.00 & 0.55 & 1.90 \\
\hline H10\% & 80145 & 6.80 & 16.50 & 0.41 & $2.7 \%$ & $12 \cdot 60$ & 0.60 & 240 \\
\hline 10110 & 50138 & 9.80 & $18 \cdot 30$ & 0.54 & 2.49 & 21.00 & 0.35 & 1.90 \\
\hline 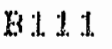 & : $: 328$ & 3.30 & 15.80 & 0.17 & $2+87$ & & 0.30 & 230 \\
\hline$B \perp 12$ & $51:=35$ & 7.10 & $26+50$ & 0.27 & 3.20 & & 1.00 & 250 \\
\hline B1. 13 & $51: 205$ & 6.70 & 29.50 & 0.23 & 2.71 & $14+10$ & 0.35 & 200 \\
\hline 8114 & 51.295 & $18+00$ & 20.20 & 0.89 & $2+30$ & 28.00 & 0.65 & 200 \\
\hline 191180 & $5 E 179$ & 3.70 & 14.30 & 0.26 & 2.49 & & & 120 \\
\hline 8116 & (1: 189 & 3.70 & 16.00 & 0.22 & $2+64$ & 8.00 & 0.90 & L: \\
\hline 8117 & 68 & $6+40$ & $21+30$ & 0.30 & 2.80 & $12+70$ & 0.60 & 160 \\
\hline B.1. & : 128 & $11: 30$ & Q & 0.27 & $3+20$ & $4 \% .00$ & 1.20 & 1.92 \\
\hline 81.9 & 8.1.1. & $1.4+60$ & $3+3: 3$ & 4.11 & $2+23$ & 30.00 & 15 & 85 \\
\hline 3120 & 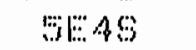 & & $3 \%+0$ & & 4.05 & & 0.40 & 250 \\
\hline $812 \%$ & $315>3$ & $1: 20$ & 27.50 & 0.19 & 2.30 & & 0.90 & 200 \\
\hline 1122 & $6 E 68$ & 4.90 & 19.60 & 0.25 & 2.48 & & 0.40 & 260 \\
\hline 8123 & 5018 & 2.100 & 10.90 & 0.23 & $2+1 \%$ & 5.20 & 0.30 & 110 \\
\hline W12A & $B E 1.8$ & 2.50 & 20.50 & 0.10 & 1.03 & & 0.70 & 80 \\
\hline E12:3 & $3 E: 58$ & 0 & 10.30 & 0 & 2.41 & & 0.60 & 40 \\
\hline 18126 & 3025 & $\therefore .30$ & $10+40$ & 0.24 & 2.22 & $1: 40$ & 0.35 & 80 \\
\hline B12\% & $5 E 8$ & $6 \cdot 70$ & $61+20$ & 0.11 & 2.63 & & 0.50 & 200 \\
\hline 3128 & 659 & 1.30 & 6.50 & 0.20 & 0.94 & 2.50 & 0.33 & 50 \\
\hline$B \perp 29$ & 8086 & 3.60 & 13.60 & 0.26 & $1.8 \%$ & 6.90 & 0.50 & 123 \\
\hline$B 130$ & 30.38 & 4.00 & 26.70 & 0.1 .5 & 2.39 & 9.20 & 0.90 & 160 \\
\hline B.3.1. & $\because 0.40$ & $4.1 \% 0$ & $12+10$ & $0.3 \%$ & 1.62 & 6.40 & 0.65 & $\because 0$ \\
\hline 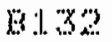 & $y=148$ & 4.00 & 1.1 .80 & 0.34 & 1.62 & 6.20 & $0.1 \mathrm{~L}: 3$ & 80 \\
\hline 81.33 & 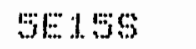 & 3.70 & 12.00 & 0.31 & 1.57 & 80.10 & 0.55 & 60 \\
\hline 8134 & 0.08 & 1.20 & $3+36$ & 0.36 & 1.39 & 3.40 & $0.8 \%$ & 36 \\
\hline 3135 & WE1.8 & 2.70 & $1.4+80$ & 0.1 .8 & 1.433 & 3.80 & 0.95 & 100 \\
\hline 81.36 & $5 E 129$ & 3.10 & 9.00 & 0.34 & 1.46 & 4.00 & 0.63 & 50 \\
\hline 5137 & 6078 & 88.00 & 230.30 & 3.41 & 2.30 & 9.00 & 0.35 & 20 \\
\hline 8.138 & 5068 & 0.90 & 20.70 & 0.04 & 1.06 & 2.70 & 0.35 & 30 \\
\hline B139 & 510.09 & 2.10 & 1.3 .30 & 0.14 & 1.45 & 3.30 & 0.60 & 30 \\
\hline [1. 140 & 5089 & 2.20 & 60 & 0.39 & 1. +20 & 60.00 & 0.90 & 30 \\
\hline 8141 & 5098 & 1.40 & 4.73 & 0.30 & 1.06 & 4.40 & $0.7 \%$ & 36 \\
\hline B. 142 & SE: 139 & 1.40 & 1.1 .00 & 0.13 & 1.40 & 3.40 & 0.75 & 1.60 \\
\hline 8143 & $867 \mathrm{~W}$ & & & & & & 0.49 & 80 \\
\hline F. 44 & $5 F 62 \mathrm{~W}$ & & & & & & 0.60 & \\
\hline$\$ \mathrm{~J} .4 \mathrm{~g}$ & $3064 \mathrm{~W}$ & & & & & & 1.40 & \\
\hline 1146 & $56.34 \mathrm{~W}$ & & & & & & $0.2 \mathrm{~g}$ & \\
\hline 8147 & $5036 \mathrm{~W}$ & & & & & & 0.45 & \\
\hline
\end{tabular}

1. See $p, A-2$. 
Granite Mountain Area

Rock Samples

\begin{tabular}{|c|c|c|c|c|c|c|}
\hline $\begin{array}{l}\text { Map } \\
\text { No. } \\
\text { Bill: }\end{array}$ & $\begin{array}{l}\text { Field No. } \\
\text { CMgA }\end{array}$ & $\begin{array}{r}4 \mathrm{ppm} \\
12.60\end{array}$ & $\begin{array}{l}\text { Th ppin } \\
45.50\end{array}$ & $\begin{array}{l}U / T h \\
0.28\end{array}$ & $\begin{array}{l}\% k 20 \\
6.86\end{array}$ & $\begin{array}{l}\operatorname{cps} \\
400\end{array}$ \\
\hline $12 \%$ & $\operatorname{CMg} \cdots .1$ & $4+20$ & :3. 30 & 0.08 & 8.90 & 400 \\
\hline X36 & CMO & 0.70 & 31.00 & 0.17 & 8.34 & 30 \\
\hline$B A F$ & CMLZ & 0 & 2.80 & 0 & 0.10 & 30 \\
\hline n: & CMII N & 0 & 2.80 & 0. & 0.27 & 1.8 \\
\hline$x \in R$ & CMIII ?...? & 0 & 0.80 & 0. & 0.31 & 1.8 \\
\hline$B \%$ & CMUONOI. & 0 & $\therefore .80$ & 0. & 0.29 & 1.7 \\
\hline B8R & CMION? & 0 & 1.30 & 0. & $0.2 \%$ & 1.7 \\
\hline 189 & CMI. & 0 & 0.0 & & 0.68 & 30 \\
\hline BIOK & CM2? 1 & 8.00 & 86.00 & 0.09 & 2.09 & i. 1.0 \\
\hline BIIIA & GM2N2? & 0.70 & 0.10 & 7.00 & 0.90 & 10 \\
\hline स1.2\% & $\mathrm{CH} 2 \mathrm{3}$ & 3.30 & 30.00 & 0.08 & 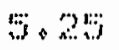 & .1. 1.0 \\
\hline $113 \%$ & CMB-1. & $2 \cdot 10$ & 9.30 & 0.23 & $3 . \% 0$ & \\
\hline Q I A $A F$ & $C M 3 \cdots 2$ & 4.80 & 39.60 & 0.12 & 8.86 & \\
\hline 0156 & $\operatorname{CMB} 3$ & $0+10$ & 0.60 & 0.80 & 0.18 & \\
\hline B. $16 \mathrm{~K}$ & $\operatorname{CMAA}$ & 1.90 & 40.80 & 0.12 & 6.93 & 280 \\
\hline Bम\% & CMA W. & 3.30 & 73.00 & 0.1 .1 & $7+3: 3$ & 600 \\
\hline $\mathrm{Blar}$ & $0 M 4 \cdots 2$ & 6.20 & 43.80 & $0+1.4$ & 4.10 & 600 \\
\hline Xis? & Com 1 & $4 \cdot 20$ & 83.10 & $0.0 \mathrm{i}$ & 5.60 & 270 \\
\hline $1908:$ & $\mathrm{CH}=3$ & $\because .90$ & 1.8 .80 & 0.11 .3 & 4.90 & 270 \\
\hline B21 & CMI 3 & 4.30 & 23.60 & 0.19 & 5.22 & 200 \\
\hline B2क & CMII 4 & 2.30 & 24.60 & 0.09 & $4.6 ! 3$ & 220 \\
\hline $82 x$ & $C M I B$ & $2 \cdot 60$ & 29.00 & 0.1 .0 & 4.92 & $2 \% 0$ \\
\hline 132 An & CMI 6 & 2.80 & 1.9 .30 & 0.1 .6 & 4.64 & 260 \\
\hline E2) & QMill & 1.00 & $1 \% \cdot 80$ & 0.06 & 4.99 & 220 \\
\hline B26R & CMII & 1.30 & 24.00 & 0.09 & 4.96 & 240 \\
\hline B27r & CMS $\cdots$ & 3.60 & $21+60$ & 0.18 & $4 \cdot 11: 3$ & 420 \\
\hline $1828 / 7$ & $C M S$ & 4.90 & 43.50 & 0.11 & 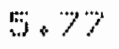 & 420 \\
\hline 199ॠ & $C M 6 \cdots 1$ & 100 & 33.30 & 0.1 .1 .3 & 6.40 & 300 \\
\hline B30F & CM6 & 3.60 & 30.30 & 0.12 & 4.27 & 300 \\
\hline $\mathrm{B} 3 \mathrm{AR}$ & CM?O & 2.60 & 21.30 & 0.12 & 4.60 & 200 \\
\hline $432 \%$ & CM2I & $\because .00$ & 18,50 & 0.11 & 4.66 & $18 \%$ \\
\hline 183314 & CMI 9 & 1. . 20 & 7.50 & 0.16 & 2.00 & 74 \\
\hline 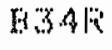 & 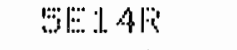 & 0. & 6.30 & 0 & 1.35 & 40 \\
\hline $836: 3$ & $\because 666 \mathrm{~F}$ & 6.80 & $1.1: 0$ & 4.63 & 0.21 & 20 \\
\hline$\sqrt{36 \%}$ & $50.64 \mathrm{~F}$ & 0.90 & 0.80 & $1 . \cdot 1.3$ & $0.2 \%$ & 20 \\
\hline $133 \%$ & WCG"SF & 3.00 & $32+100$ & 0.1 .8 & 3.20 & 190 \\
\hline k38\% & $506 ?$ & 2.40 & $13+00$ & 0.18 & $\because+20$ & 200 \\
\hline 8396 & \%0.6.17A & 4.40 & 19.30 & 0.23 & 8.60 & 200 \\
\hline WAOF & $50611 \mathrm{E}$ & 2.70 & 1200 & 0.22 & 6.23 & 200 \\
\hline BAIF & $H F=1$ & 1.2 .0 & 7.80 & 0.1 .4 & 2.09 & 1. 150 \\
\hline $842 \mathrm{k}$ & $4 F 5$ & 」. .50 & 9.30 & 0.16 & 2.60 & 1100 \\
\hline \$AB & $H F=3$ & 3.00 & 45050 & 0.18 & 7.00 & 150 \\
\hline 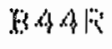 & $1060 F$ & 2.90 & $10 \cdot 30$ & 0.28 & 3.12 & \\
\hline W A WF & WGOOFA & 1.20 & 80 & 0.21 & $6 \%+89$ & \\
\hline 84627 & 60:59 & $\therefore 40$ & $12+130$ & 0.19 & 8.70 & 200 \\
\hline$X 4 \% F$ & :30: & 4.00 & 39.00 & 0.11 & 3.34 & 200 \\
\hline $148 \mathrm{R}$ & $H \cdots 1 \cdots 1$ & 2.60 & 27.30 & 0.10 & 4.20 & $33 \%$ \\
\hline $649 \mathrm{R}$ & $\| \cdots \cdots$ & 4.90 & 9.30 & 0.63 & 3.86 & \\
\hline Bю्̈ & $|\cdots|: \cdot 1 . \cdots 3$ & 5.00 & $62+60$ & 0.08 & 2.66 & \\
\hline 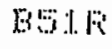 & 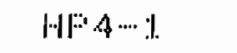 & 7.90 & $2 \% \cdot 130$ & $0.3 \%$ & 8.40 & 390 \\
\hline
\end{tabular}




\begin{tabular}{|c|c|c|c|c|c|c|}
\hline $\begin{array}{l}\text { Map } \\
\text { No. } \\
\text { BS.2R }\end{array}$ & $\begin{array}{l}\text { Field No. } \\
\text { HFA-2 }\end{array}$ & $\begin{array}{l}\text { U ppm } \\
6.50\end{array}$ & $\begin{array}{l}\text { Th ppm } \\
56.30\end{array}$ & $\begin{array}{l}\mathrm{U} / \mathrm{Th} \\
0 . \mathrm{L} 2\end{array}$ & $\begin{array}{l}\% K_{2} \mathrm{O} \\
9.15 \\
\end{array}$ & CPS \\
\hline BS $35 \mathrm{Fi}$ & $H F \cdot 4-3$ & 4.00 & $2 \mathrm{~J}$ & 0.18 & 7.00 & \\
\hline BSAR & 40.3 & 6.50 & 31.50 & 0.21 & 6.55 & 420 \\
\hline [5 & $H F 2-1$ & 6.60 & 34.60 & 0.19 & 0.75 & 460 \\
\hline ESG & $H F 2$ & 7.20 & 36.50 & 0.20 & 6.21 & \\
\hline$[A 57 R$ & $H F$ & 6.00 & 33.80 & 0.1 .8 & $6.83: 3$ & \\
\hline$B 58 F$ & $H F--4$ & 4.70 & 38.90 & 0.12 & 7.75 & \\
\hline $\mathrm{BSSF}$ & $H F^{\prime} Y-1$ & 4.50 & 39.30 & 0.11 & 8.21 & 550 \\
\hline $\mathrm{BGOF}$ & HP>ー" & 38.00 & 54.50 & 0.51 & 4.00 & \\
\hline H61.7i & HF है। 1 & 8.40 & 32.50 & $0+26$ & 5.92 & 390 \\
\hline $862 \%$ & $H F B-7$ & 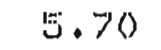 & $40 \cdot 30$ & 0.1 .4 & 6.95 & \\
\hline E63\% & HF 10 & 3.30 & 32.60 & 0.10 & 3.43 & 300 \\
\hline $\mathrm{B} G A \mathrm{~A}$ & $H P \perp 1$ & 2.40 & 43.80 & 0.05 & 3.40 & 250 \\
\hline $665 R$ & 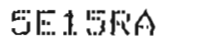 & 4.20 & 23.50 & 0.1 .8 & 2.80 & 325 \\
\hline E665 & 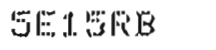 & 4.00 & 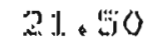 & 0.19 & $6 \cdot 64$ & \\
\hline W675 & SC7.1. & 1.60 & 3.00 & 0.53 & 1.41 & 50 \\
\hline BEBF & SEIGRA & $7 \cdot 30$ & 28.830 & 0.28 & 6.80 & 2.15 \\
\hline B69li & EEDGRB & 0.90 & $\delta .80$ & 0.13 & 7.75 & \\
\hline BYOR & $M F 12$ & 3.70 & 23.30 & 0.36 & 1.20 & 250 \\
\hline B $71 \mathrm{~F}$ & $H F^{3} 1.3$ & 3.50 & 25.00 & 0.1 .4 & 4.45 & 220 \\
\hline $132 \pi$ & $M F \perp A$ & 2.60 & 22.80 & 0.11 & 1.78 & 225 \\
\hline घ3\%3F & $Q C 1$ & 0.70 & 1.60 & 0.47 & 0.68 & 25 \\
\hline $18>4 R$ & $Q C^{2} 2$ & 1.00 & 2.00 & $1 . .00$ & 2.41 & 30 \\
\hline B>ジ̈: & $\mathrm{arc} 3$ & 3.10 & 14.30 & 0.1 .6 & $0.31: 3$ & 40 \\
\hline B> & QR. 4 & 1.3 & 0 & & (). As? & 30 \\
\hline 8775 & RCS & 0.90 & 3.30 & 0.207 & $0 . \omega$ & 48 \\
\hline II 78 & $R C 6$ & 1.50 & 6.00 & 0.298 & 0.50 & 40 \\
\hline 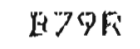 & QLO & 1.90 & 3.30 & 0.58 & 0.30 & \\
\hline S180F & QCB & 0.40 & 1.80 & 0.23 & 0.35 & 25 \\
\hline KEIF & QC9 & 0.40 & $1 . .80$ & 0.22 & $0.2 \%$ & 30 \\
\hline प182F & QC:LO- & 2.00 & 3.30 & 0.6 .1 & $0.7 \%$ & 100 \\
\hline MS3F & acilO- 2 & .1 .70 & 7.80 & 0.22 & 3.40 & \\
\hline$M \# 4 x^{\prime}$ & RCLO- & 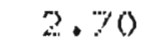 & $1: 3.00$ & 0.18 & 5.72 & 100 \\
\hline IBESF & $0010 \cdots 1$ & 3.10 & 9.830 & $0.3 \%$ & 3.06 & 100 \\
\hline ME6R & (ac) & 3.00 & $22=80$ & 0.1 .3 & 4.837 & 200 \\
\hline $58 \% R$ & (UC 1.2 & 3.00 & 20.80 & $0.1 . A$ & 4.30 & 150 \\
\hline I:33Fi & AC I & 0. & 3.00 & 0 & 0.63 & 30 \\
\hline $\operatorname{BSOR}$ & QRCD.4-2] & 0 & 1.20 & 0. & 0.26 & \\
\hline IS9OF & $5057 \ltimes$ & 6.10 & $2 \%$ & $0.2 \%$ & 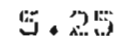 & 1. 20 \\
\hline Ey $1 \mathrm{~K}$ & GH1 & 1.50 & 0.683 & $2+2 \%$ & 0.83 & 283 \\
\hline BS' $2 R$ & $G M_{2}=$ & 1.80 & 0.78 & 2.31 & 0.02 & 24 \\
\hline EST $3 F$ & GM $2 A$ & $2 \cdot 30$ & .1 .67 & 1.383 & 1.08 & \\
\hline$\left[394 F^{\circ}\right.$ & GM:3A & 1.70 & 0.43 & 2.70 & 0.23 & 1.20 \\
\hline ESS & GM:3B & 1.70 & 2.60 & 0.65 & $0.3 \%$ & \\
\hline By $6 \mathrm{~F}$ & $6 M 30$ & 2.30 & 3.65 & 0.63 & 0.20 & \\
\hline 3078 & GM3n & 1.50 & 4.43 & 0.34 & 0.79 & \\
\hline LOEF & GMZEE & 6.20 & $4 \mathrm{~L} \cdot \mathrm{J} .0$ & 0.19 & 7.00 & \\
\hline $\mathrm{BSOF}$ & GM $3 F$ & 6.20 & 45.80 & 0.14 & 7.71 & \\
\hline W.OSB & GM30 & 6.80 & 42.70 & 0.16 & 7.50 & 120 \\
\hline$E I O R$ & KMA & $\mathrm{J} .70$ & 30.20 & 0.06 & 5.02 & 250 \\
\hline 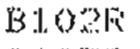 & Gis & 0.80 & 22.60 & 0.04 & 5.32 & 250 \\
\hline BII. OJ $\mathrm{F}^{\circ}$ & GMS & 0.60 & 17.40 & 0.03 & 3.83 & 250 \\
\hline EIOAF & GiM7 & 1.60 & 50.60 & 0.03 & $6+00$ & 3130 \\
\hline
\end{tabular}




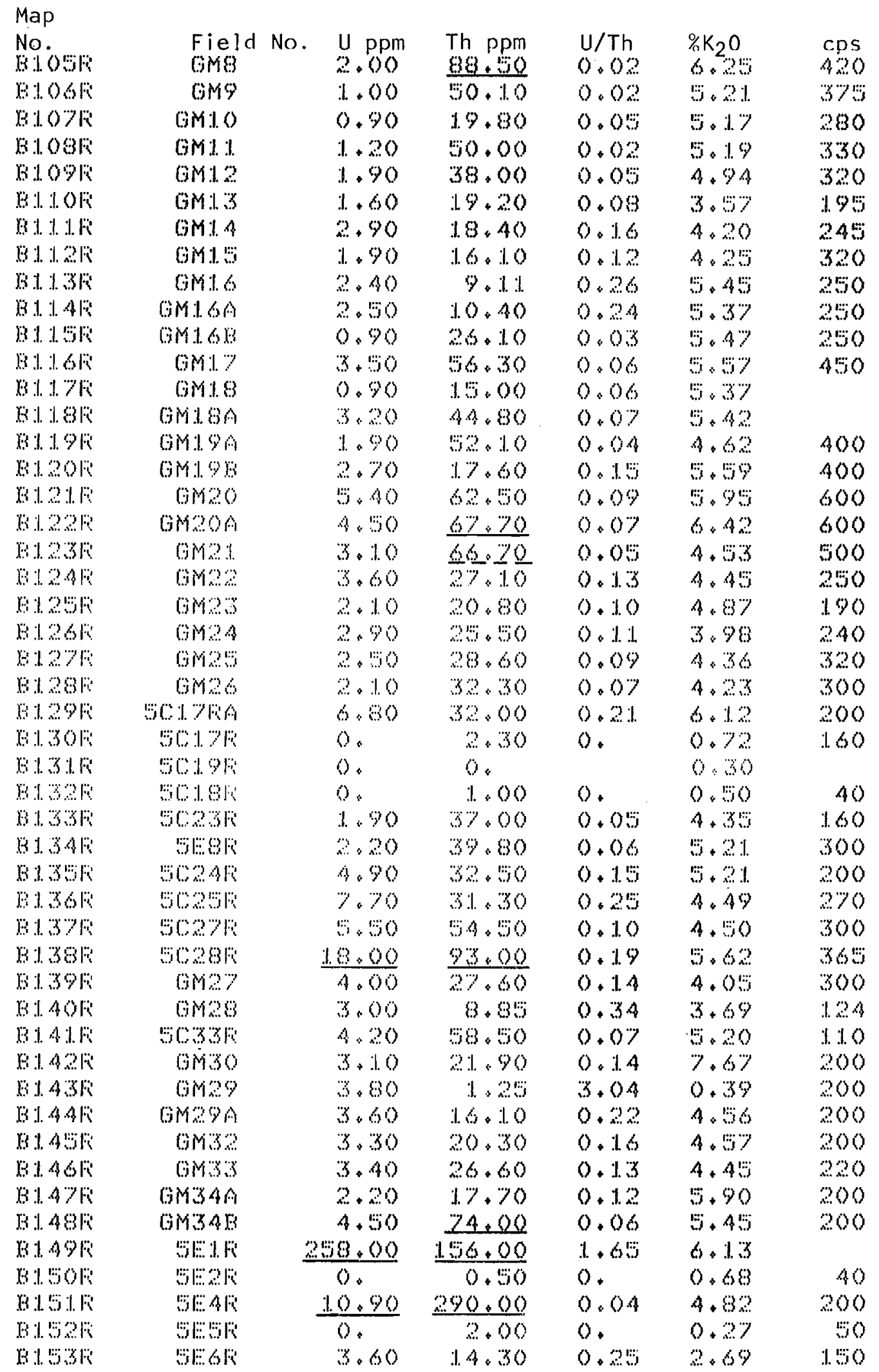


The Darby Mountains

Sediment and Water Samples

\begin{tabular}{|c|c|c|c|c|c|c|c|c|}
\hline $\begin{array}{l}\text { Map } \\
\text { No. }\end{array}$ & Field No. & $\begin{array}{l}\text { U PPM } \\
\text { (RAA)I }\end{array}$ & Th ppm & U/Th & $\% K_{2} O$ & $\begin{array}{l}\text { U Ppm } \\
(\text { LASL)I }\end{array}$ & $\begin{array}{l}\mathrm{H}_{2} 0 \mathrm{ppp} \\
(\mathrm{LASL})\end{array}$ & cPs \\
\hline A.1 & 561689 & 1.30 & 13.50 & 0.10 & 1.67 & 5.20 & 0.80 & 80 \\
\hline$A 2$ & 50.1705 & 250 & 10.20 & 0.25 & $1+31$ & 4.20 & & \\
\hline A 3 & 507715 & 1.20 & 9.44 & 0.1 .3 & $1.5 \%$ & & & \\
\hline A.4 & 501725 & 2.10 & 9.4 .4 & 0.2 .2 & 1.75 & & & 60 \\
\hline$A 5$ & 561735 & 2.00 & 1.0 .70 & $0.1 \%$ & $\because .57$ & & & 80 \\
\hline$A B$ & 601675 & 0.80 & 6.383 & 0.1 .3 & 1.37 & $\therefore \& 830$ & 0.30 & \\
\hline$A 7$ & 501669 & 2.80 & 15.60 & 0.18 & 3.82 & 3.90 & 0.50 & 80 \\
\hline A 8 & $36+698$ & 24.00 & 10.0 & 1.49 & 2.67 & $30.0(0)$ & 2.00 & 250 \\
\hline 09 & $5 C 1635$ & 8.50 & 28.90 & 0.30 & 3.87 & 18.00 & 0.90 & 250 \\
\hline ا.lll. ل & $5 \mathrm{CH} 62 \mathrm{~S}$ & $17 \cdot 10$ & 20.40 & 0.84 & 2.47 & 25.00 & & 100 \\
\hline$\sqrt{2}$ & 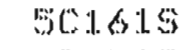 & 7.00 & $3,3.20$ & 0.21 & 2.84 & 12.80 & & 100 \\
\hline (J) & 502515 & 12.00 & 36.50 & 0.33 & 2.41 & $1.8 \cdot 30$ & 0.60 & 80 \\
\hline$J A$ & $5 C 1505$ & 3.30 & 16.10 & 0.20 & 1.72 & 4.50 & 0.40 & 60 \\
\hline ن & 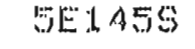 & 13.40 & $28 \cdot 60$ & 0.47 & $2 \cdot 13$ & 1.3 .90 & $\underline{1} \cdot \underline{B}$ & \\
\hline 16 & $5 \mathrm{C} 1495$ & 28.00 & 27.00 & ง. .04 & 2.10 & 49.00 & 1.055 & 1.00 \\
\hline .17 & $50 \mathrm{~B} \$ 85$ & 10.30 & 4.1 .20 & 0.17 & $\therefore .31$ & 21.00 & 0.40 & 100 \\
\hline (1) & $5 E$ I 478 & $\underline{33}, 00$ & $61 \cdot 20$ & 0.87 & 2.59 & 30.00 & & \\
\hline 19 & 5181.165 & iis. $0(0)$ & 25.00 & 2.04 & 2.65 & $4 ! 3.00$ & J. .00 & \\
\hline ald 0 & $50 \perp 578$ & $16+40$ & 8.4 .70 & 0.1 .8 & 3.45 & 32.00 & & .350 \\
\hline .1. I. J. & 501585 & 11.60 & 19.10 & 0.61 & 2.74 & 1.3 .90 & & 250 \\
\hline 112 & 501605 & $? 1.00$ & $\because 4,30$ & 0.72 & 1. .49 & 43.00 & & 250 \\
\hline 53 & 501959 & 220 & 20.40 & 0.11. & 3.78 & 7.20 & & \\
\hline .111 & 401035 & 4.30 & 9.70 & 0.44 & 1.71 & 6.60 & 1.05 & 150 \\
\hline J J & 501338 & 5.40 & 26.30 & 0.21 & 2.99 & 9.90 & 0.80 & 150 \\
\hline . 16 & 61498 & I. I. . 30 & 1.4 .20 & 0.80 & $1.4 \%$ & 3.20 & & 300 \\
\hline .117 & $5 E 1485$ & 11.30 & $7.7 x$ & 1.46 & 1.42 & 3.60 & 1. . 25 & \\
\hline 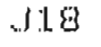 & 601558 & 1.60 & 9.18 & $0.1 \%$ & 1.52 & 3.00 & 0.45 & \\
\hline .119 & 50.1543 & $15 \cdot 80$ & 6.12 & 2.55 & 2.800 & 20.00 & 1.05 & \\
\hline 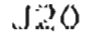 & 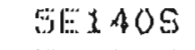 & 曹 80.00 & 43.80 & 1.32 & $2+64$ & $5 \% .00$ & 1. 15 & 600 \\
\hline M? & $8=3.396$ & 举. 00 & 67.80 & 0.75 & 2.74 & $73+00$ & & 600 \\
\hline . (3)"? & 5 C 1458 & $31 .>0$ & 11.4 .00 & 0.71 & 2.78 & $\underline{1.11 .00}$ & 0.60 & 450 \\
\hline 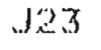 & $5 E 145$ & 4.20 & 62.00 & 0.07 & 1.95 & & & 400 \\
\hline 124 & $5 E A d A$ & 28.00 & 28.10 & 2.78 & 2.37 & & & 400 \\
\hline .125 & $5 C: 1465$ & 些9.00 & 130.00 & 0.45 & 3.25 & 2.3 .00 & L. 45 & 300 \\
\hline 126 & GE 1 A ? & 6.90 & 21.40 & 0.32 & 1.38 & 6.70 & 1.35 & 1.20 \\
\hline . 127 & $5 E: 433$ & 8.30 & 24.00 & 0.35 & 1.93 & & 1.33 & 200 \\
\hline $.12 B$ & 501.475 & 24.20 & $16 \cdot A 0$ & 0.52 & 2.70 & 62.00 & 0.95 & 300 \\
\hline . & 50.436 & 6) $1 . \Omega(2)$ & 96.90 & 0.66 & $2+77$ & 78.00 & 0.30 & 3() 0 \\
\hline . 130 & 501425 & 20.00 & 74.00 & 0.27 & 2.95 & & & 300 \\
\hline $.33 \%$ & $5 E\rfloor 385$ & a. 20 & $A d \cdot 30$ & 0.54 & 3.3 .3 & $22+00$ & & \\
\hline J32 & 501300 & 35.00 & 56.00 & 0.63 & 3.08 & 50.00 & 1.90 & $\therefore 40$ \\
\hline 13.3 & 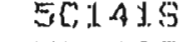 & 52.00 & 53.50 & 0.97 & 2.55 & 1.3 .70 & 2.30 & 300 \\
\hline .334 & 50.409 & 47.00 & 46.50 & 1.01 & 2.94 & & & 300 \\
\hline 134 & 501338 & 37.00 & 57.10 & 0.68 & 3.45 & 36.00 & 2.05 & 200 \\
\hline$\sqrt{1.36}$ & $5 E 1329$ & 21.00 & 28.10 & 0.75 & $2+10$ & 30.00 & 1.25 & 300 \\
\hline 137 & $5 E 1,3,36$ & 1. 5.40 & 25.50 & 0.60 & 1.88 & 20.00 & & 200 \\
\hline .138 & $5 E 1346$ & 49.00 & 1.9 .90 & 2.46 & 2.10 & 49.00 & 1.15 & 350 \\
\hline .139 & 50,346 & 20.00 & 46.50 & 0.43 & 3.26 & 29.00 & 1.70 & 300 \\
\hline .140 & 50135.3 & $15+90$ & 79.50 & 0.20 & 3.31 & 37.00 & 1.6 & 300 \\
\hline .51 & 5E1:36 & $3 A .00$ & 40.30 & $0.8 \%$ & 3.27 & 22.00 & 2.20 & 300 \\
\hline 142 & 31305 & 40.00 & 53.10 & 0.75 & 2.39 & 37.00 & 0.60 & \\
\hline 143 & 501319 & 19.10 & 72.00 & 0.27 & 2.393 & 36.00 & 1.65 & 250 \\
\hline
\end{tabular}

1. See P, A-2. 


\begin{tabular}{|c|c|c|c|c|c|c|c|c|}
\hline $\begin{array}{l}\text { Map } \\
\text { No. } \\
1 / 44\end{array}$ & $\begin{array}{l}\text { Field No. } \\
\text { yl: } 208\end{array}$ & $\begin{array}{c}\text { U ppm } \\
(\mathrm{RAA})^{1} \\
1 \% .00\end{array}$ & $\begin{array}{l}\text { Th ppm } \\
40.80\end{array}$ & $\begin{array}{l}\mathrm{U} / \mathrm{Th} \\
0.42\end{array}$ & $\begin{array}{l}\% \mathrm{~K}_{2} \mathrm{O} \\
2 \mathrm{I}: \mathrm{H}\end{array}$ & $\begin{array}{l}\text { U ppm } \\
(L A S L) \\
42.00\end{array}$ & $\begin{array}{l}\mathrm{H}_{2} \mathrm{O} \quad \mathrm{ppb} \\
(\mathrm{LASL}){ }^{1} \\
1.30\end{array}$ & cps \\
\hline $14 \%$ & "ש:1295 & 36.00 & 46.90 & $0.7 \%$ & 2.49 & 43.00 & & \\
\hline .146 & 701296 & 17.30 & 61.00 & 0.28 & 2.60 & & & 100 \\
\hline $.14 \%$ & 801309 & 4.00 & 64.00 & 0.76 & 2.44 & 33.00 & & $\because 00$ \\
\hline 140 & जE 12\% & 20.90 & 41.30 & 0.49 & 2.30 & 33.00 & 0.95 & 400 \\
\hline .149 & : 1166 & 23.50 & 37.80 & 0.62 & 2.12 & 36.00 & 0.40 & 400 \\
\hline .150 & 5181.8 & 5.00 & .19 .90 & 0.26 & 1. .86 & & & $42 \mathrm{~g}$ \\
\hline . & 601328 & 29.00 & $60 \cdot 60$ & 0.46 & 2.40 & 38.00 & $1+23$ & 300 \\
\hline $1 \% 2$ & 561335 & 99.00 & 73.70 & 0.80 & $2+31$ & 45.00 & $1+15$ & 200 \\
\hline $.5 \% 3$ & 54256 & 27800 & 40.00 & 0.168 & 2.36 & 30.00 & 1.60 & 300 \\
\hline .154 & 801269 & 30.00 & 11.00 & 2.73 & 1.23 & 40.00 & 1.00 & 300 \\
\hline $.10 \%$ & 501289 & 9.80 & $42+10$ & 0.23 & 1.92 & 45.00 & 0.40 & 400 \\
\hline .156 & 601278 & 29.00 & $9 \% .00$ & 0.29 & 2.34 & & & 100 \\
\hline $4 \%$ & 61248 & 33.00 & 36.00 & 0.38 & 2.48 & 41.00 & 1.30 & 100 \\
\hline 198 & $5 f 1258$ & 13.90 & 66.00 & 0.21 & 2.88 & 32.00 & $1+65$ & 300 \\
\hline $1 \% 9$ & 60189 & 36.00 & $4 \% .00$ & 0.73 & 2.06 & 3.00 & $0.5: 5$ & 2100 \\
\hline 160 & 501239 & 1060 & $7 \% .90$ & 0.72 & $2+64$ & & & 300 \\
\hline $16 \%$ & $31: 1248$ & $22 \cdot 60$ & 91.80 & $0.21 \%$ & J. $.8 \%$ & & 1.96 & 400 \\
\hline 162 & $\because 12+20$ & 64.00 & 30.30 & $2+11$ & 3.26 & 100.00 & 1.16 & 230 \\
\hline .63 & $\because 12,235$ & 49.00 & $36 \%$ & 1.37 & 2.46 & 04.00 & & 300 \\
\hline .164 & 601200 & 23.00 & $62=00$ & 0.44 & 1.86 & 37.00 & 0.99 & 1500 \\
\hline .163 & (i) 118 & 33.00 & 62.20 & 0.53 & 3.69 & & 1.68 & 400 \\
\hline 166 & $5018 \%$ & $\because 30$ & $2 \times 31$ & 0.46 & 1.69 & & & 600 \\
\hline 167 & 361849 & 6.70 & $6 \% .80$ & 0.10 & 4.83 & & & 600 \\
\hline 168 & 601839 & 1.4 .60 & 1.49 .100 & 0.10 & 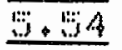 & & & 600 \\
\hline .169 & $61: 21529$ & 10.40 & 18.20 & 0.30 & 1.85 & & & 600 \\
\hline 170 & $9 E 1006$ & 9.90 & $47 \cdot 60$ & $0.2 \%$ & $2.7 \%$ & & & 600 \\
\hline 171 & : & 0 & $16 \cdot 10$ & 0. & 2.93 & & & 600 \\
\hline $1 \%$ & 10198 & $2 \% .00$ & 121.40 & 0.63 & 3.30 & & & 600 \\
\hline $17 \%$ & 501818 & 3.70 & 70.40 & 0.08 & 2.83 & & & 600 \\
\hline 174 & 613.36 & $3 . \% 0$ & 1.4 .40 & 0.26 & $1.8 \%$ & & & \\
\hline $17 \%$ & 61809 & 0.80 & 1.70 & $0+1$ & 2.46 & & & 600 \\
\hline $1 \%$ & $61: \cdots 48$ & 3.70 & 16.00 & $0.2 \div 3$ & 2.16 & & & \\
\hline $.17 \%$ & 6009 & 3.80 & 31.10 & 0.19 & $4=16$ & 7.10 & 1.00 & 160 \\
\hline .178 & 80916 & 7.40 & 26.30 & 0.28 & 9.70 & 11.00 & 0.45 & 110 \\
\hline .179 & 1687 & 6.30 & $20 \cdot 20$ & 0.31 & 3.26 & 10.20 & $0+28$ & 200 \\
\hline .180 & 30098 & 7.110 & 10.60 & 0.68 & 1.86 & 8.40 & & 1.50 \\
\hline .181 & : & 6.70 & 1.4 .90 & 0.45 & $.8 \%$ & 9.00 & $0.8 \%$ & 160 \\
\hline .182 & 26898 & 7.70 & $2 x \cdot 10$ & 0.3 .3 & $3.7 \%$ & $1 x+10$ & 0.39 & 200 \\
\hline .183 & $\because E 906$ & 9.20 & $26+30$ & 0.35 & $3+41$ & 18.30 & 0.33 & 200 \\
\hline 184 & 60,86 & 8.10 & 1.60 & 0.3 .3 & 1. $\%$ & 7.70 & 0.75 & 160 \\
\hline $.18 \%$ & 50848 & 7.30 & 22.00 & 0.3 .3 & 3.23 & 16.40 & 0.80 & 1.60 \\
\hline .186 & 30368 & $8+20$ & 24.50 & 0.33 & $2+86$ & & & 200 \\
\hline .167 & $\because 6916$ & 8.60 & $60+50$ & 0.1 .4 & 3.06 & 14.10 & 1.45 & 300 \\
\hline .180 & 315926 & 9.90 & 28.90 & 0.21 & $2.9 \%$ & 1.3 .60 & 0.25 & 300 \\
\hline 189 & $\because 4929$ & 6.80 & 37.00 & 0.18 & 2.72 & 9.80 & 0.80 & 2100 \\
\hline .190 & 50945 & 3.30 & 32.70 & 0.12 & 3.1 .1 & 10.20 & 0.40 & 200 \\
\hline .191 & 680 & 8.90 & 60.60 & 0.1 .4 & 3.20 & $12 \cdot 20$ & 0.50 & 200 \\
\hline .192 & "BE & 9.30 & 20.90 & 0.10 & 3.69 & 26.00 & & 1200 \\
\hline .193 & 6209 & 0.50 & 36.00 & 0.24 & 3.36 & d. 1.60 & 0.70 & \\
\hline .194 & 6838 & 6.90 & 33.40 & $0+21$ & 4.03 & 9.30 & 0.90 & 300 \\
\hline .195 & 6073 & $4 \cdot 1: 0$ & 14.00 & 0.32 & $2.1 \%$ & & 1.80 & 200 \\
\hline .196 & 6078 & 2.70 & $33+90$ & 0.08 & 2.79 & 10.80 & 0.95 & 200 \\
\hline
\end{tabular}

1. See p. A-2. 


\begin{tabular}{|c|c|c|c|c|c|c|c|c|}
\hline $\begin{array}{l}\text { Map } \\
\text { Noy }\end{array}$ & Figld No. & $\begin{array}{l}U \text { ppm } \\
\left(\mathrm{RAA}^{1}\right)^{1}\end{array}$ & $T_{34}: 88$ & $8 / T h$ & $\frac{6}{3} 28$ & $\begin{array}{l}\text { U ppm } \\
\left({ }_{0} \text { ASL }\right)^{l}\end{array}$ & $\begin{array}{l}\mathrm{H}_{2} \mathrm{O} \\
(\text { LASL) }\end{array}$ & 200 \\
\hline .198 & $60 \% 63$ & $3+40$ & 30.60 & 0.18 & 3.98 & 9.00 & 0.80 & 200 \\
\hline .199 & $30 \% 8 \%$ & 8.30 & $60 \cdot: 30$ & 0.1 .4 & 3.90 & & $0.9 \%$ & 300 \\
\hline J. I. & $40 \% \%$ & 1.80 & 93.00 & 0.04 & 4.60 & 1. 1.70 & & 290 \\
\hline J1. & $10 \% 98$ & 6.00 & 33.20 & 0.18 & 3.84 & 10.10 & 0.40 & 250 \\
\hline 1102 & 60809 & 6.70 & 36.00 & 0.1 .9 & 3.90 & 11.20 & 0.35 & 300 \\
\hline J103 & 6081.8 & 6.30 & 32.00 & 0.20 & 3.90 & 9.80 & 1.75 & 250 \\
\hline 1104 & 60025 & $3+20$ & 78.80 & $0.0 \%$ & 3.04 & & & 200 \\
\hline J10\% & $\because 0.36$ & 6.30 & $3 \% .20$ & 0.1 .4 & 4.18 & 9.60 & 0.35 & 220 \\
\hline .1106 & $: 1: 896$ & 4.20 & 20.20 & 0.21 & 4.60 & 5.70 & 0.30 & 1.79 \\
\hline $.110 \%$ & !: & 2.90 & 12.30 & 0.24 & 3.36 & 7.30 & 0.00 & 200 \\
\hline 1108 & $6: \div 86$ & $1: 10$ & 26.60 & 0.19 & 3.59 & 6.80 & 0.20 & 200 \\
\hline $.110 \%$ & 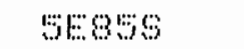 & 0.40 & 24.20 & 0.02 & 9.20 & $\%: 40$ & 0.25 & 200 \\
\hline . III I. & $6:=845$ & 0. & $1.8+1.0$ & 0 & 3.29 & 7.90 & 0.23 & \\
\hline 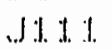 & 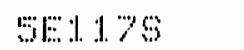 & 0.40 & 10.60 & $0.63 \mathrm{~L}$ & 2.20 & 11.10 & 1.25 & 200 \\
\hline 11.2 & : & 3.60 & 4.4 .3 & 0.79 & 2.09 & 8.40 & 1.30 & 120 \\
\hline 1113 & FI: I L 68 & 8.60 & 18.80 & 0.30 & $2+05$ & 9.20 & 0.40 & \\
\hline . 111.4 & 61205 & 2.10 & 6.60 & 0.32 & 2.94 & 7.60 & 1.05 & \\
\hline $111 \%$ & 91.98 & 4.00 & 7: & 0.53 & 2.20 & 8.90 & 1.25 & \\
\hline . I. 1.6 & $\because 01129$ & 4.70 & 1.1. .00 & 0.42 & 2.06 & 8.40 & 0.45 & \\
\hline J11\% & 91169 & 6.60 & 14.60 & 0.46 & 3.13 & 1. . . .90 & 0.60 & \\
\hline II I I 8 & 301139 & 60 & 16.10 & 0.36 & $2+21: 3$ & 9.60 & 0.95 & \\
\hline 1119 & $\because 01196$ & 8.60 & $2 \mathrm{~J} .70$ & 0.39 & 1. 82 & 1.3 .00 & $L, L$ & \\
\hline J & $90 \mathrm{I} 195$ & 6.60 & 94.00 & 0.08 & $\therefore \cdot 16$ & $3 \% .00$ & 1.35 & \\
\hline Jा & 601189 & 4.30 & $1 \% .60$ & 0.24 & 2.63 & $8 \cdot 10$ & $1+8=$ & \\
\hline J1.28 & 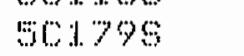 & 4.60 & 10.60 & 0.44 & $2+18$ & & & 200 \\
\hline . 1123 & $61 \% 89$ & 3.00 & 9.10 & 0.33 & 2.17 & & & 200 \\
\hline 1.94 & 601745 & 4.90 & 1.3 .30 & 0.37 & 1. .67 & & & 200 \\
\hline J1.28 & $6017 \%$ & $4+60$ & 10.20 & 0.44 & 2.22 & & & 200 \\
\hline$J 126$ & $61 \% 1765$ & 3.70 & 8.42 & 0.44 & 2.39 & & & 200 \\
\hline J12\% & $601 \% 79$ & 3.80 & $8 \cdot 16$ & 0.47 & 2.110 & & & 200 \\
\hline 1120 & $80 \%$ & 3.70 & $9 . \% 0$ & 0.38 & 2.89 & 6.90 & $1.6 \%$ & 80 \\
\hline 1129 & FE89 & $2 . \%$ & 24.70 & 0.1 .1 & 1.84 & $\because: 70$ & 0.35 & \\
\hline 130 & $8 \%$ & 4.70 & 12.60 & 0.38 & 1.94 & 10.20 & 0.60 & 150 \\
\hline 1131 & FIF: & $\because, 40$ & 60.00 & 0.09 & 2.34 & 12.00 & 0.50 & 200 \\
\hline 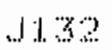 & 9896 & 3.90 & $60 .: 00$ & 0.10 & 2.38 & $\therefore .4 .40$ & & 180 \\
\hline $4 \pi 3$ & HEg6 & 0 & 32.30 & 0. & 2.48 & 1. 4.80 & & \\
\hline 134 & BE J 000 & 8.00 & 26.00 & 0.1 .9 & 2.74 & & & 475 \\
\hline 1136 & $5 E 945$ & 20.00 & 4.70 & $4 \cdot 26$ & 1.69 & 23.00 & 0.79 & 200 \\
\hline 1136 & 601.18 & 4.00 & $2 L \cdot 20$ & 0.1 .9 & 3.69 & 7.40 & 0.93 & 100 \\
\hline 1137 & WE I L 6 & 3.60 & $14+40$ & $0.2 \%$ & 0.76 & 8.10 & $0.6 \%$ & \\
\hline . 1.38 & 1. 45 & 3.00 & 13.80 & 0.22 & 2.34 & 5.80 & $0.6 \%$ & 1.00 \\
\hline 1139 & $5 E 11.36$ & 0.90 & 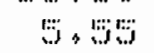 & 0.1 .6 & 2.68 & 8.40 & 0.53 & 100 \\
\hline 1140 & & 2.60 & 3.43 & 0.48 & 2.83 & $\because .80$ & 0.90 & 160 \\
\hline 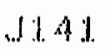 & 501085 & 2.00 & 7.1 .4 & 0.28 & 3.73 & 4.40 & $1+9$ & 100 \\
\hline 1142 & 60.1069 & 3.60 & $1 \% .90$ & 0.20 & 2.75 & 9.90 & 1.60 & 200 \\
\hline 1143 & 601078 & 3.80 & $2 \div 80$ & 0.15 & 4.08 & $10+20$ & 0.90 & 200 \\
\hline 1144 & $\because E A I S$ & 6.60 & 10.70 & 0.62 & 2.64 & 10.40 & 1. .35 & \\
\hline . 1148 & BEd d. & 9.00 & 6.68 & 1.37 & 3.24 & 12.40 & 0.25 & \\
\hline 11.46 & :I: 1089 & 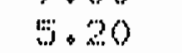 & 10.70 & 0.49 & 2.46 & 7.20 & $0.6 \%$ & 200 \\
\hline J1. $4 \%$ & $1: 1095$ & 4.40 & 12.90 & 0.34 & 2.49 & 7.10 & 0.30 & \\
\hline 11.48 & 601045 & 1. . $\%$ & 20.40 & 0.08 & 2.93 & 6.90 & 0.90 & $1: 30$ \\
\hline 1149 & $5610 \%$ & 6.80 & $1.2+30$ & 0.64 & 2.44 & 9.20 & 0.10 & 1.60 \\
\hline
\end{tabular}

1. See p. A-2. 


\begin{tabular}{|c|c|c|c|c|c|c|c|c|}
\hline $\begin{array}{l}\text { Map } \\
\text { No. } \\
\text { J150 }\end{array}$ & $\begin{array}{l}\text { Field No. } \\
\text { GE106s }\end{array}$ & $\begin{array}{l}\text { U Ppm } \\
(\text { RAA } ! \\
7.1 .0\end{array}$ & Th 890 & $\begin{array}{l}\text { l//Th } \\
0.24\end{array}$ & $\begin{array}{l}8 \mathrm{~K}_{2} \mathrm{O} \\
2.30\end{array}$ & 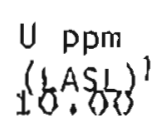 & $\begin{array}{l}\mathrm{H}_{2} \mathrm{O} \text { ppo } \\
\left(\mathrm{LASL}^{\prime}\right)^{1}\end{array}$ & 998 \\
\hline 1.51. & ::-1.035 & $1 \% .20$ & 12.00 & 1.02 & 2.07 & 11.80 & $0 . \Delta 0$ & 100 \\
\hline 1152 & 5 & 5.50 & 9.20 & 0.60 & 1.60 & 93,70 & 0.23 & 120 \\
\hline d15.3 & $5 \% 1025$ & 4.60 & 1.3 .50 & 0.34 & 1.34 & 10.30 & 0.00 & 120 \\
\hline$\sqrt{ } 15$ A $_{3}$ & k: 10.10 & 5.90 & 1.3 .20 & $0.4 k j$ & $2 \cdot 1.1$ & 10.00 & 0.15 & \\
\hline . 15.5 & $5 E 1045$ & 1.1 .70 & 13.60 & 0.86 & 1. 0.9 & 12.70 & 1.05 & 1.20 \\
\hline $1 \geq 56$ & $5 C L() 25$ & 9.30 & 26.80 & 0.35 & 2.17 & 14.90 & 0.20 & 100 \\
\hline 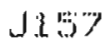 & 501035 & 9.60 & 33.70 & $0.28\}$ & 2.35 & 13.90 & 0.65 & 200 \\
\hline J & $5[1.015$ & 9.00 & 20.20 & 0.45 & 2,34 & 12.70 & 0.70 & 100 \\
\hline 3153 & 103595 & 7.30 & 1.7 .1 .0 & 0.43 & 2.835 & & & 300 \\
\hline 51.60 & 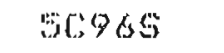 & 5.10 & 27.90 & 0.28 & 2.77 & $8 \cdot 30$ & 0.60 & 1.00 \\
\hline .31 .61 & $\because 6085$ & 8.10 & 1.8 .10 & 0.43 & 2.27 & 1. 1.20 & 0.85 & 100 \\
\hline $16 \%$ & $50 \%$ & $8.0\rangle$ & 21.40 & $0.3 \%$ & 2.58 & 9.00 & 0.70 & 100 \\
\hline $\int \log x$ & 5095 & 5060 & 18.70 & 0.30 & 2.91. & & & 0 \\
\hline
\end{tabular}

The Darby Mountains Rock Samples

\begin{tabular}{|c|c|c|c|c|c|c|}
\hline \multicolumn{7}{|l|}{ Map } \\
\hline No. & Field No. & U ppm & Th ppm & U/Th & $\% \mathrm{~K}_{2} \mathrm{O}$ & cps \\
\hline Jit & :1:295 & 49.70 & 51.00 & $0.3 \%$ & 4.95 & 300 \\
\hline .2\% & DAA $3 \cdots \cdots$ & 0 & 3.00 & 0. & 1.57 & 160 \\
\hline J'Ki & UAAX & 0.50 & $20 \cdot 30$ & $0.0 \%$ & 0.47 & 160 \\
\hline (1) $A B$ & IIA4X-3 & 0 & 0.62 & 0. & 0.71 & 160 \\
\hline $15 F$ & IIAA $43 \cdots$ & 0.90 & 11.90 & 0.08 & $\because 30$ & 1.60 \\
\hline JSF & [IAAS & 2.60 & $56 \cdot 70$ & 0.05 & 4.50 & 600 \\
\hline J"lit & IAAB & 8.70 & $33 \cdot 30$ & 0.1 .7 & $4 \cdot 43$ & 660 \\
\hline.$M B F$ & $M A A Y-1$ & 8.20 & 91.00 & 0.16 & 4.37 & \\
\hline .194 & $\ln 4 \% \%$ & 3.00 & $1.5 \cdot 20$ & 0.16 & 4.45 & \\
\hline 山৬ぬ & 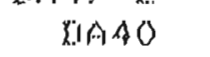 & 4.50 & 35.90 & 0.1 .3 & 1.94 & 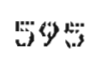 \\
\hline fli x. Fi & [IAA1. & 2.80 & $463+40$ & $0.0 \mathrm{~S}$ & 4.45 & 600 \\
\hline WI: & IIA $48-1$ & 5.80 & 64.30 & 0.09 & $4 \cdot 42$ & 610 \\
\hline Jis & $Y A A B-2$ & $? . \% 0$ & 57.90 & 0.0 & 4.92 & \\
\hline J18R & 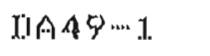 & 8.80 & 24,50 & 0.36 & 8.39 & \\
\hline WLF & UA $49 \ldots$ & 8.830 & $16 \cdot 60$ & 0.5 .5 & 5.043 & 520 \\
\hline $116 \mathrm{Fi}$ & 0450 & 4.80 & 46,30 & 0.1 .0 & 5.17 & 540 \\
\hline JI.7R & OA & 2.90 & 44.20 & 0.07 & 4.32 & 600 \\
\hline $18 F$ & ডEЗフк内 & 13850 & 32,80 & 0.41 & 5.08 & $62 \%$ \\
\hline $.519 F$ & $\operatorname{lin} 34-1$ & 1.3 .50 & 13.50 & 0.27 & 4.33 & 520 \\
\hline WOF & $\operatorname{lin} 34-2$ & 5.10 & $32 \cdot 30$ & 0.16 & 6.18 & 520 \\
\hline 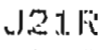 & 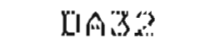 & 7.30 & $4 A .20$ & $0.1 \%$ & 5.02 & 500 \\
\hline J22R & 21438 & 2.80 & 19.80 & $0.1 A$ & 0.62 & 460 \\
\hline .235 & DA:B:3A & 9.80 & $19 . \% 0$ & 0.50 & 3.76 & 920 \\
\hline$=124 F$ & IIMA4: & 7.90 & 13.50 & $0.5 \%$ & $4.7 \%$ & 13.50 \\
\hline 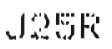 & DIA4A & $3: 00$ & $2 \%$ & 0.18 & 4.04 & 475 \\
\hline$M G$ & DА:З & 10.40 & 14.60 & 0.78 & 4.86 & 180 \\
\hline . & $\operatorname{liA} 39$ & $t \cdot 30$ & 44.80 & 0.03 & $4.0 \%$ & 580 \\
\hline 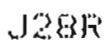 & $11 A 27 A$ & 2.10 & 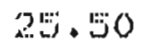 & 0.08 & 4.01 & 375 \\
\hline J29F & DA2 8 & 4.50 & 29.70 & 0.15 & 4.39 & 420 \\
\hline Jore & MAZINN & $4 \cdot 80$ & $28 \cdot 60$ & 0.17 & $4.88^{\prime \prime}$ & 320 \\
\hline J 318 & $\operatorname{nas} 30$ & $\therefore .30$ & $238 \cdot 10$ & 0.08 & 4.96 & 375 \\
\hline
\end{tabular}




\begin{tabular}{|c|c|c|c|c|c|c|}
\hline Map & & & & & & \\
\hline $\mathrm{No}_{32 \pi}$ & $\begin{array}{l}\text { Field No. } \\
\text { IIA: } 3: 1-1\end{array}$ & 2. Ppg & Th. Bgh & $y / T_{0,3}$ & $3 \times 289$ & -ps \\
\hline 5336 & InAZ\...' & 1.70 & 5.21 & 0.3 .3 .3 & 0.70 & 40 \\
\hline J J & $5 \mathrm{CL} \perp 29 \mathrm{~K}$ & 10.00 & 48.50 & 0.21 & 4.58 & 900 \\
\hline 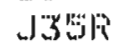 & 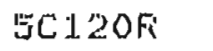 & 160 & 43.50 & 0.13 & 5.25 & 500 \\
\hline$J 36 \mathrm{~F}$ & SCLIIR & 6.70 & 42.50 & 0.16 & 4.85 & 400 \\
\hline $53^{\circ 77}$ & SEZ 396 & 7.00 & 0.70 & 10.00 & 0.08 & \\
\hline$J 38 R$ & I.C8-1. & 6.40 & 34.90 & 0.18 & $12 \cdot 04$ & \\
\hline $339 R$ & $\operatorname{ICg} \theta-2$ & 5.10 & 42.70 & 0.1 .3 & 21.83 & \\
\hline 1405 & $n c 8-3$ & 5,40 & 56.30 & 0.10 & 11.92 & \\
\hline JAIF & $D C 5-1$ & 5.40 & $72=40$ & 0.07 & 9.21 & 580 \\
\hline$J A 2 F$ & $n \subset 5-2$ & 4.50 & 31.50 & 0.14 & 9.16 & 580 \\
\hline 14.35 & $\operatorname{IrC} 4$ & 9.00 & 44.830 & 0.20 & 11.18 & 570 \\
\hline$J \& 4 \%$ & $\operatorname{IC} 1$ & 7.50 & 67.70 & 0.11 & 10.65 & 570 \\
\hline $345 A$ & {$[1,2 \ldots 1$} & 4.80 & 71.40 & 0.07 & 10.35 & 700 \\
\hline$J 46 F$ & nC:-2 & .1. 1.00 & $92+30$ & 0.13 & 9. & 700 \\
\hline.$\triangle A 7 K$ & $002-3$ & 20.30 & 66.10 & 0.3 .1 & 10,77 & 700 \\
\hline . $48 B$ & $\ln c x-1$ & 7.40 & 46.90 & 0.1 .6 & 1.0 .34 & 600 \\
\hline 1498 & $\operatorname{sic} 3-2$ & 5.40 & $41 \cdot 10$ & 0.13 & 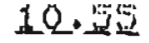 & 600 \\
\hline$J 50 R$ & $206-1$ & 4.30 & 62.50 & 0.07 & 10.47 & \\
\hline J518 & {$[1 C \&-2$} & 5.90 & 34.80 & 0.17 & 11.1 .3 & \\
\hline $.52 \%$ & IICG-3 & 3.90 & 68.10 & 0.06 & $11+3.3$ & \\
\hline J5" & {$[\operatorname{le} 7-1$} & 9.00 & 76.00 & 0.12 & 6.3 .3 & \\
\hline J & {$[10>\cdots 2$} & 10.00 & 64.60 & 0.15 & 7.09 & \\
\hline & {$[\operatorname{lic} 7 \cdots 3$} & 7.30 & 68.30 & 0.13 & 6.97 & \\
\hline $.36 \%$ & $5 E 23 R$ & & & & & \\
\hline $.5 \%$ & $|A\rangle$ & 2.10 & 14.00 & 0.15 & 7.80 & 270 \\
\hline J5GR & AA & 1.90 & 10.830 & 0.18 & 6.65 & 140 \\
\hline .1596 & {$[/ A B-1$} & 7.80 & 65.00 & 0.11 & $4.9 \%$ & 225 \\
\hline 1606 & $\square A B-2$ & 3.50 & 73.50 & 0.05 & 4.60 & 225 \\
\hline ل & $\operatorname{SAA} 8 \cdots 3$ & 2.830 & 89.50 & 0.05 & 1.70 & 225 \\
\hline 1638 & $\angle A B-A$ & 4.30 & 28.00 & $0.1 \%$ & 6.10 & 225 \\
\hline J63א & ПАВ - . & 9.60 & 8360.60 & 0.11 & 4.84 & 225 \\
\hline$J 64 \sqrt{2}$ & DA9A & 5.70 & 43.50 & 0.13 & 4.86 & 275 \\
\hline J6:5i' & IIAS世 & 2.70 & 13.50 & 0.20 & 7.80 & 275 \\
\hline$\sqrt{6} 6 \mathrm{~K}^{2}$ & UAIIO-1. & 7.20 & $5 \% .50$ & 0.13 & 5.10 & 500 \\
\hline J67\% & LIA].0-? & 8.00 & 64.00 & 0.1 .3 & 5.00 & 500 \\
\hline $568 R$ & $\operatorname{IA10\cdots 3}$ & 6.80 & 47.00 & 0.10 & A. 20 & 500 \\
\hline J保它 & $\eta A \pm 0-A$ & 7.10 & 27.58 & 0.09 & 4.10 & 500 \\
\hline J\%OR & IIAN II & 0. & 8.00 & 0 & 4.60 & 120 \\
\hline $.171 \mathrm{R}$ & DAG & $\therefore 30$ & 26.10 & 0.07 & 7.00 & 240 \\
\hline J" & $\operatorname{IIASA} 1$ & 4.30 & 47.00 & 0.09 & 8.70 & 250 \\
\hline J7.3R & [HASA? & 3.00 & O) & 0.1 .4 & 8.96 & 250 \\
\hline $.574 F$ & $\prod A 5 A 3$ & 20 & 200 & 0.09 & 7.60 & 250 \\
\hline $\mathrm{J} 75 \mathrm{R}^{2}$ & UA & 2.00 & 27.00 & 0.07 & 5.67 & 280 \\
\hline$J 76 F^{\circ}$ & [IAS …2] & 5.20 & 42.00 & 0.12 & 8.90 & 280 \\
\hline 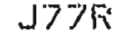 & [1A5-3 & 2.10 & 37.00 & 0.06 & 7.1 .5 & 250 \\
\hline 578R' & $\operatorname{LA} 1$ & 2.20 & 30.00 & 0.07 & 4.46 & 320 \\
\hline Ј79Fi & $\operatorname{LAIIA}$ & 0 & 7.30 & 0 & 2.60 & 70 \\
\hline J80Fi & $\square A \perp 2 E$ & 0. & 21.00 & 0. & 3.23 & 70 \\
\hline$J 8: L$ & $5 E^{2} 4 F_{i}$ & 4.00 & 36.00 & 0.11 & 6.49 & 200 \\
\hline $.182 \mathrm{~K}$ & MA2A & 0 & 7.00 & 0. & 0.02 & \\
\hline J833F" & โIA2स & 0.90 & 1.0 .80 & 0.08 & $5.5 \%$ & \\
\hline J84F' & $\triangle A O 2 C$ & 2.30 & 13.30 & 0.17 & 4.60 & \\
\hline
\end{tabular}




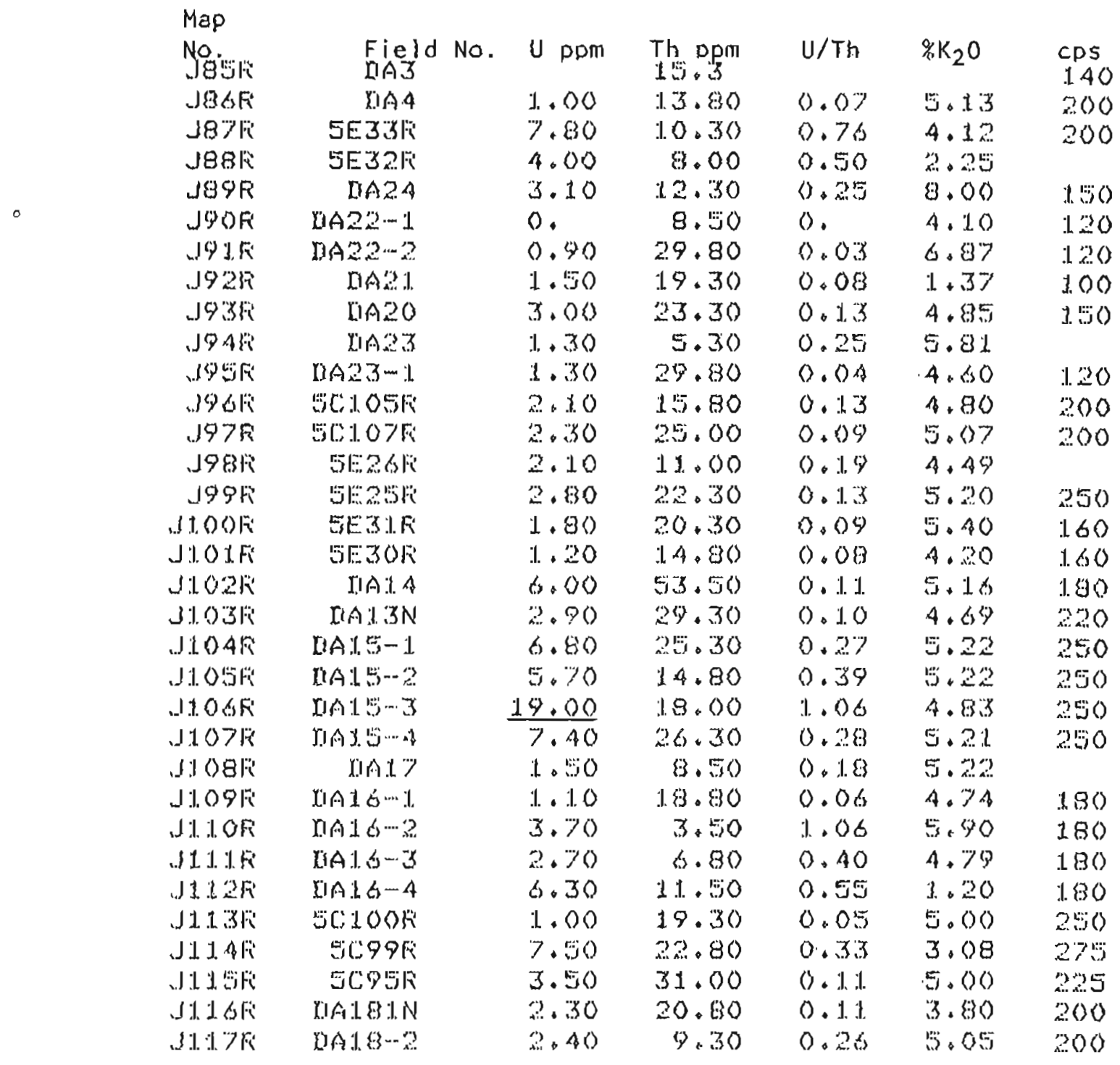

Selawik Hills Area

Sediment and Water Samples

\begin{tabular}{|c|c|c|c|c|c|c|c|c|}
\hline $\begin{array}{l}\text { Map } \\
\text { No. }\end{array}$ & Field No. & $\begin{array}{l}\text { U pom } \\
(R A A) l\end{array}$ & Th ppm & U/Th & $\% K_{2} 0$ & $\begin{array}{l}\text { U ppm } \\
(L A S L) 1\end{array}$ & $\begin{array}{l}\mathrm{U} \text { Ppb } \\
(L A S L)\end{array}$ & cps \\
\hline 91 & $5 E 2425$ & $\mathrm{~J} .70$ & 10.00 & 0.17 & $1.4 \xi 3$ & 3.90 & 0.20 & \\
\hline$\theta+2$ & $5 E 2406$ & 1.60 & 3.50 & 0.4 .3 & 1.20 & 3.30 & 0.36 & \\
\hline 83 & $5=2418$ & 1.80 & 5.00 & 0.36 & 2.00 & $x .80$ & 0.55 & \\
\hline 64 & $5 E 2 A 4 S$ & 0.90 & 5.00 & 0.1 .83 & 1.73 & 1.00 & 0.20 & \\
\hline Q5i & $5 E 2 A 35$ & 2.20 & 1.50 & 1.47 & 1. .20 & 4.90 & 0.00 & \\
\hline 106 & $5 E 2488$ & 1.40 & 1.00 & 1.40 & 0.98 & $?: 10$ & 0.65 & 60 \\
\hline 67 & $5 E .2998$ & 1.60 & 14.50 & 0.10 & 0.93 & 1.80 & 0.25 & \\
\hline 68 & 3152476 & 2.50 & 14.50 & 0.17 & $1.21: 3$ & 3.60 & 0.55 & \\
\hline G9 & SE:3AS & 1.30 & 6.00 & 0.22 & $1+26$ & $2,6,0$ & 0.45 & \\
\hline 010 & EEX35S & 1.10 & 7.50 & 0.15 & 1.93 & 3.20 & 0.150 & \\
\hline
\end{tabular}

i. See p. A-2. 


\begin{tabular}{|c|c|c|c|c|c|c|c|c|}
\hline $\begin{array}{l}\text { Map } \\
\text { No. } \\
\text { liti. }\end{array}$ & $\begin{array}{l}\text { Field No, } \\
31: 2375\end{array}$ & $\begin{array}{l}\text { U ppm } \\
(\text { RAA) } 1 \\
4.70\end{array}$ & $\begin{array}{l}\text { Th ppm } \\
\text { I. } 3.60\end{array}$ & $\begin{array}{l}\text { U/Th } \\
0.33\end{array}$ & $\begin{array}{l}\% \mathrm{~K}_{2} 0 \\
1.3 \%\end{array}$ & $\begin{array}{l}U \mathrm{Ppm} \\
(\text { LASI.) }\end{array}$ & $\begin{array}{l}U \text { ppb } \\
(\text { LASL) } 1 \\
0.0 \% 0\end{array}$ & $\begin{array}{c}\text { CPS } \\
300\end{array}$ \\
\hline B.2 & 85:2589 & 1.00 & 10.00 & 0.40 & 7.23 & 10.00 & 1.20 & \\
\hline 01.3 & $51: 2368$ & 1.1 .0 & $20 \cdot 50$ & $0.0 \mathrm{~s}$ & 1.31 & 3.10 & 0.50 & 30 \\
\hline 6. 4 & 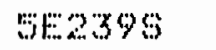 & 1.10 & 8.00 & 0.1 .4 & 1.72 & 3.50 & 0.40 & \\
\hline 01.5 & 512946 & $2 .: 100$ & 10.00 & 0.100 & 1.33 & 4.80 & 0.113 & \\
\hline 016 & $5 E 2465$ & 1.00 & 1.5000 & 0.07 & 1.61 & 4.20 & 0.40 & \\
\hline $61 \%$ & $91: 2636$ & 1.30 & 1.00 & 1.30 & 1.43 & 2.70 & 0.15 & \\
\hline 6.18 & 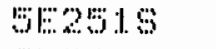 & $1 . .70$ & 2.50 & 0.68 & 1. . 3 & & 0.10 & \\
\hline $61 \%$ & $51=2: 309$ & 1.50 & I. I. . 00 & 0.1 .4 & 1.20 & $3+1.0$ & 0.93 & \\
\hline 820 & 61818 & 3.10 & 20.60 & 0.15 & 2.76 & 5.50 & 0.35 & \\
\hline $62 \%$ & i: 1809 & 3.80 & 30.70 & 0.1 .2 & 3.38 & 7.00 & 0.80 & 160 \\
\hline 622 & WE: 1795 & 7.60 & 92.70 & 0.08 & 3.79 & 37.00 & 0.45 & 150 \\
\hline 623 & 61828 & 6.60 & 96.90 & $0.0 \%$ & 4.00 & 31.00 & 0.60 & \\
\hline 624 & WE:1768 & 5.50 & 35.70 & 0.1 .3 & 3.84 & 21.00 & 0.00 & 400 \\
\hline $02 \%$ & 36175 & 7.60 & $41 \cdot 10$ & 0.18 & 3.28 & 1.1 .00 & 0.05 & 600 \\
\hline 026 & : & 5.70 & $48 \cdot 40$ & $0+1.2$ & $3+2 \%$ & 1.8 .00 & 0.15 & \\
\hline $82 \%$ & 561778 & 7.40 & 27.10 & $0.2 \%$ & 3.40 & 9.3 .00 & 0.10 & \\
\hline 628 & 31789 & $1 \% .70$ & 36.40 & 0.1 .6 & $2+80$ & 11.50 & $0.3 \%$ & \\
\hline 029 & 618183 & 0.00 & 37.00 & 0.22 & $27+87$ & 10.60 & 0.00 & \\
\hline 630 & 581896 & 6.80 & $62+60$ & 0.1 .1$. & 3.21 & 1.3 .00 & 0.1 .3 & 200 \\
\hline 031 & $5818 \%$ & $8 \%+30$ & $22+60$ & $0+10$ & 3.39 & 10.90 & 0.60 & $4: 10$ \\
\hline 032 & :1:1898 & 4.60 & $7 A+10$ & 0.06 & 4.85 & 26.00 & & 400 \\
\hline 0.3 & 6181899 & 7.40 & $36+30$ & 0.1 .3 & 2.98 & 1.9 .90 & 0.00 & 300 \\
\hline 634 & $51: 1919$ & 3.30 & $6 \% \cdot 20$ & 0.09 & 2.86 & 16.20 & 0.63 & 400 \\
\hline 836 & $51: 1909$ & 7.40 & 34.20 & 0.14 & $2+88$ & 27.00 & & 400 \\
\hline 836 & 95.923 & 13.50 & 71.40 & 0.08 & 3.66 & 17.00 & 0.15 & $1 \div 0$ \\
\hline 837 & 51893 & 3.60 & $16 \cdot 60$ & 0.23 & $2+21$ & 6.00 & 1.05 & 200 \\
\hline 038 & 61:-938 & 2.30 & $26 .: 30$ & 0.09 & 2.49 & 7.10 & 0.45 & 260 \\
\hline 639 & $61520 \%$ & 4.40 & 76.00 & 0.06 & $\therefore .80$ & & & \\
\hline 640 & $31: 2089$ & 8.70 & 48.00 & 0.18 & $3+67$ & & & \\
\hline 641 & $31: 2095$ & 4.80 & 62.00 & 0.08 & 3.3 .3 & & & \\
\hline 042 & $61=21.6$ & 9.30 & 69.60 & 0.13 & 3.26 & & & \\
\hline$\$ 43$ & GE.2106 & 10.10 & 41.00 & 0.28 & 4.02 & & & \\
\hline 644 & $1: 2049$ & 0.50 & $17+30$ & 0.03 & 3.67 & & & \\
\hline 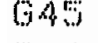 & 352039 & 7.00 & 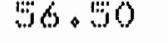 & $0+12$ & 3.76 & & & \\
\hline 046 & $81: 201.9$ & 0.60 & 22.00 & 0.02 & 3.100 & & & \\
\hline $14 \%$ & GE:1945 & 3.60 & 29.00 & 0.12 & 2.42 & 7.20 & 0.40 & 200 \\
\hline 6.48 & GE.995 & 0. & $18+00$ & 0 & 2.00 & & & 180 \\
\hline 0.49 & 8102005 & 1.90 & 44.60 & 0.04 & 3.44 & & & 250 \\
\hline 650 & $61: 1989$ & 3.70 & 11.60 & 0.32 & 2.20 & & & 150 \\
\hline $95 \mathrm{~L}$ & $5 E 1965$ & 1.40 & $13+50$ & 0.10 & 1. . 71 & & & 1.80 \\
\hline 062 & i: 1978 & 3.30 & 47.00 & 0.07 & $3.4 \%$ & & & $1: \% 0$ \\
\hline 633 & 6338 & 2.20 & 27.00 & 0.08 & 3.1 .6 & & & 120 \\
\hline 634 & $3: 32978$ & 3.80 & $22 \cdot 1: 0$ & 0.17 & 2.50 & 9.50 & 0.35 & \\
\hline 693 & $31: 2285$ & 2.20 & $32+00$ & 0.07 & 3.12 & 8.50 & & \\
\hline 036 & $31: 295$ & 6.40 & $6 \%+: 00$ & 0.10 & 3.19 & 16.20 & 0.40 & \\
\hline 897 & 362315 & $4 \cdot 30$ & 7.100 & 0.57 & 2.30 & 6.70 & 0.40 & \\
\hline 638 & 62328 & 2.70 & $8 .: 30$ & 0.32 & $2+20$ & 6.70 & 0.70 & \\
\hline $0 \%$ & al: 1978 & 5.60 & 13.30 & 0.41 & 1.83 & & & 150 \\
\hline 660 & il:.:. 1.998 & 5.00 & 43.60 & 0.11 & 2.08 & & & \\
\hline 661 & HE. 1980 & 4.40 & 26.30 & 0.17 & 2.36 & & & \\
\hline 062 & 615018 & 11.80 & 95.00 & 0.1 .2 & 3.28 & & & 250 \\
\hline
\end{tabular}

1. See p. A-2. 


\begin{tabular}{|c|c|c|c|c|c|c|c|c|}
\hline $\begin{array}{l}\text { Map } \\
\text { No. }\end{array}$ & Field No. & $\begin{array}{l}U_{\text {ppm }} \\
(R A A)\end{array}$ & Th opm & U/Th & $\% \mathrm{~K}_{2} \mathrm{O}$ & $\begin{array}{l}\text { U Ppm } \\
(L A S L)\end{array}$ & $\begin{array}{l}U p p b \\
(L A S L)\end{array}$ & cos \\
\hline $66 x$ & SE2OSG & 0 & 1.1 .00 & & & & & 80 \\
\hline E64 & تE.2OG & 5.50 & $32 \cdot 00$ & 0.17 & 2.87 & & & \\
\hline Gos & $5 E 204 \mathrm{~B}$ & 6.00 & 35.00 & 0.17 & 2.70 & & & \\
\hline 666 & SEE'SOS & 10.70 & 45.50 & 0.24 & 4.03 & 1.7 .00 & 0.10 & \\
\hline 067 & 5EZ19S & 15.00 & 26.00 & 0.58 & 1.65 & 2.1 .00 & 0.70 & \\
\hline G68 & $5 E 2215$ & 7.30 & 70.00 & 0.10 & 2.06 & .4 .70 & 0.15 & \\
\hline G\&9 & $5 E 2225$ & $1.0,1.0$ & 32.50 & $0.3 \%$ & 2.95 & 14.60 & 0.35 & \\
\hline 070 & SE218S & 7.30 & 37.50 & 0.19 & 2.48 & 11.00 & 0. & 200 \\
\hline 071 & $51: 2235$ & 4.50 & 59.50 & 0.08 & 3.84 & & 0.00 & \\
\hline G\%" & $5 E 2245$ & 13.90 & 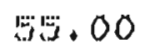 & 0.29 & 3.70 & $\triangle A=00$ & 0.50 & 300 \\
\hline$G>3$ & $51: 2259$ & $27+40$ & 32.00 & 0.86 & 3.04 & $1.00,00$ & 0.20 & \\
\hline 671 & 5E20\% & 11.50 & 32.00 & 0.36 & $3.3 \%$ & & & \\
\hline 675 & SE2J IS & $L(1) .50$ & 36.50 & 0.29 & 2.46 & 110 & $1+25$ & 200 \\
\hline 676 & 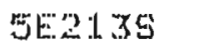 & 11.70 & 31.00 & 0.38 & 2.28 & 2.00 & 1.55 & 200 \\
\hline 077 & $5 E 2155$ & $9 . \$ 0$ & .28 .30 & 0.131 & 3.45 & 14.50 & 1.21 .1 & 200 \\
\hline $6 \% 8$ & 51.45 & 9.70 & 15.00 & 0.51 & 2.30 & 8.60 & 1.75 & 200 \\
\hline G\%9 & 5E2175 & 2.50 & 17.00 & 0.28 & 1. .5.3 & 3.20 & & 200 \\
\hline 1080 & $5 E 2165$ & 10.20 & 52.50 & 0.15 & 3.20 & 32.50 & 0.30 & \\
\hline G81 & 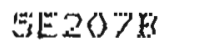 & 9.10 & $3 x-00$ & 0.26 & 3.22 & & & \\
\hline 682 & 5 辰1 679 & 2.30 & 4.10 & 0.56 & 1. .20 & 2.70 & 0.35 & \\
\hline 683 & SE1.695 & 2.40 & 5.49 & 0.40 & 0.90 & 2.40 & 0.35 & 30 \\
\hline QSA & $5 E 170 S$ & 3.30 & 7.78 & 0.43 & 1.09 & 3.90 & 0.60 & 30 \\
\hline
\end{tabular}

I. See $p . A-2$.

Selawik Mills Area

Rock Samples

\begin{tabular}{|c|c|c|c|c|c|c|}
\hline \multicolumn{7}{|l|}{ Map } \\
\hline No. & Field No. & U pprit & Th $\mathrm{ppm}$ & U/Th & $\mathrm{sK}_{2} \mathrm{O}$ & cps \\
\hline Gin & $A F=$ & 2.70 & $15+60$ & 0.1 .7 & $14.7 \%$ & 425 \\
\hline (6) & $A F A$ & 7.80 & 44.80 & 0.17 & 1.4 .36 & 360 \\
\hline 0.35 & $A F 3$ & 6.60 & 44.80 & 0,15 & 1.4 .8 .4 & 350 \\
\hline GAF & $A P 2-1$ & 1.50 & 11.70 & 0.04 & $1 \% .19$ & 250 \\
\hline Geir & $A F=2-2$ & 2.20 & 26.00 & 0.08 & 13.50 & 250 \\
\hline$G 6 F^{\prime}$ & $A F \perp-1$ & 1.00 & 16.10 & 0.08 & 17.05 & 250 \\
\hline G\%R & $A F 1-2$ & 1.50 & 27.10 & 0.06 & 1.6 .49 & 250 \\
\hline 0815 & $A F B A$ & 86.00 & 70.30 & 1. .2.2 & $1.0 .61: 3$ & 200 \\
\hline G9R & $A F G B$ & 9.60 & 8.33 & $x+25$ & $1.1 \cdot 87$ & 510 \\
\hline $510 F^{\circ}$ & $A P B C$ & 92.00 & $3 \% .00$ & 2). 49 & I. 1.1 .3 & 900 \\
\hline GII Fi & $A P B$ & 5.60 & 45,80 & 0.12 & 7.77 & 400 \\
\hline GJ.2R & $A F>$ & 6.40 & 42.70 & 0.15 & 6.48 & 360 \\
\hline (3. $3 \mathrm{~F}$ & APIL 1 & 3.40 & $20 \cdot 830$ & 0.16 & 6.94 & 110 \\
\hline Gil $4 \mathrm{R}$ & $A P B$ & 3.30 & 21.40 & 0.15 & 8.37 & 220 \\
\hline 61.58 & $A F^{\prime}: 10-1$ & 1.6 .20 & $5 \% .00$ & 0.32 & 7.46 & 720 \\
\hline GldR & $A F: 10-2$ & 1.5 .70 & 54.20 & 0.29 & 8.20 & 720 \\
\hline G178 & $5 F \cdot 27$ & 0 & 22.30 & 0. & 4.00 & 410 \\
\hline GI8F & $5 F 28$ & 2.10 & 24.30 & 0.10 & 4.17 & 250 \\
\hline $6.19 \%$ & $5 F=29-1$ & 1.60 & 20.30 & 0.08 & 5.22 & 500 \\
\hline $620 R$ & $5 F 29-23$ & 5.10 & 31.50 & 0.1 .6 & 7.83 & 500 \\
\hline GIA & $5+9-3$ & 3.10 & 31.50 & 0.10 & 6.40 & 500 \\
\hline
\end{tabular}




\begin{tabular}{|c|c|c|c|c|c|c|}
\hline $\begin{array}{l}\text { Map } \\
\text { No. } \\
\text { G? }\end{array}$ & $\begin{array}{l}\text { Field } \\
\text { SFS }\end{array}$ & No. & Th, $\mathrm{Ppm}$ & U/Th & 8.80 & ${ }_{2}^{2} \cdot 8 \%$ \\
\hline G.23F & $S F A$ & 3.10 & 24.50 & 0.13 & 6.5 .3 & 200 \\
\hline โัม & $S F \cdot 3$ & 1.70 & 10.00 & 0.17 & 7.65 & 260 \\
\hline G25F & $S F 1-1$ & 2.00 & 4.50 & 0.44 & 5.18 & 240 \\
\hline G2EF & $S F \perp-2$ & 2.00 & 25.80 & 0.08 & 4.55 & \\
\hline Q27K & $5 F 1-3$ & 1.10 & 17.30 & 0.06 & 6.39 & \\
\hline $028 \%$ & $S F^{\prime} 2$ & 0. & 5.80 & 0 & 5.84 & 265 \\
\hline Q29F & $5 \% 6$ & 3.50 & 21.30 & 0.16 & 5.65 & 250 \\
\hline G30F & $5 F \%-1$ & 0.80 & 3.00 & 0.27 & 5.30 & 230 \\
\hline $331 F$ & $S F \cdot 7-2$ & 3.10 & 23.80 & 0.21 & 5.30 & 230 \\
\hline Q3.5F & 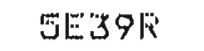 & 0.60 & 7.00 & 0.09 & 7.8 .3 & 425 \\
\hline G3.35i & 13 COOOR & 0 & $? 250$ & 0 & 5.66 & 400 \\
\hline $634 \%$ & تC.1.991" & 9.00 & 70.50 & 0.13 & 6.96 & 400 \\
\hline $035 \%$ & 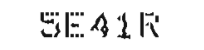 & 7.90 & 4.30 .00 & 0.02 & 8.50 & \\
\hline G36 & 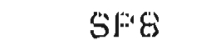 & 3.20 & 21.50 & 0.15 & 6.48 & 200 \\
\hline $637 \%$ & $5 F 9$ & 6.50 & 33.00 & 0.20 & 6.45 & \\
\hline 03817 & SEAS西 & 6.00 & $2 I .00$ & $0.2 \%$ & 6.37 & 250 \\
\hline 6.398 & $S P 12$ & 3.50 & (7).30 & 0.38 & $4.7 \%$ & 250 \\
\hline $640 F^{2}$ & SF'. J. & 7.70 & $2\} 3.30$ & 0.27 & 7.08 & 700 \\
\hline GA $\mathrm{AF}$ & $8 \nLeftarrow 1 \zeta-1$ & 4.40 & 40.00 & $0.1 \mathrm{~J}$. & 6.80 & 600 \\
\hline KA2) & $5 F+3$ & 6.70 & 23.30 & $0.26 s$ & $5+44$ & 400 \\
\hline C. 436 & $5 F 2 A \cdots 1$ & 0. & $23 \cdot 50$ & 0 & 7.77 & 310 \\
\hline GAAF & $5 \% 24$ & 3.50 & $56+50$ & 0.06 & 2.25 & 310 \\
\hline Q4 & $81: 36$ & 1.70 & $33 \cdot 30$ & 0.05 & 7.60 & 23 \\
\hline (i) 4 Gi & $8 F 25$ & 0. & 7.50 & 0 & .1 .00 & 220 \\
\hline (3) $47 F$ & $6 F 23$ & 8.40 & 21.50 & 0.39 & 1.34 & 300 \\
\hline (.) 3831 & $S 1^{*}: 9$ & 1.60 & 27.30 & 0.06 & 1. $1 .+70$ & 270 \\
\hline 6497 & $5020-1$ & 0. & J. . . 50 & 0 & .12 .40 & 210 \\
\hline$G^{2}=0,0$ & $5120-2$ & 0. & 9.50 & 0. & 13.80 & 21.0 \\
\hline G5].F & $6 F-1-1$ & 0. & 6.00 & 0. & 9.114 & 200 \\
\hline G举2月 & $5 F 21-7$ & 0 & 6.50 & 0 & 9.60 & 260 \\
\hline $6 \pi 38$ & $8 \% 2$ & $13,59,00$ & 618.00 & 0.22 & 7.20 & 780 \\
\hline C: AR & $5 \% 22 A 2$ & 44.00 & 277.50 & 0.16 & 7.10 & 780 \\
\hline 05 & $5 \% 2281$ & 1.70 & 8.80 & 0.1 .9 & 8.48 & 780 \\
\hline $656 \pi$ & $5 \% 22 \mathrm{~B} 2$ & 12.20 & 1.7 .30 & 0.07 & 6.05 & 780 \\
\hline G & GF 20 & $23+60$ & 2. J. 80 & 0.1 .2 & 10.40 & 580 \\
\hline $658 \pi$ & $S F \perp B-1$ & 0.0 & 0.0 & & & 30 \\
\hline $6591: 8$ & $5 \% 18-2$ & 0. & 1. .70 & 0. & 0.3 .1 & 30 \\
\hline GoO & $5 F \perp \theta-3$ & 0.0 & 0.0 & & $0+5$ & 30 \\
\hline GG]R & SF.1.7 & 2.40 & 25.50 & 0.09 & 5.00 & 250 \\
\hline GBZR & GEA.3R & 4.60 & 16.50 & 0.28 & 6.44 & 250 \\
\hline Cobi3k & $5 E A E F$ & 0.70 & 0.40 & 1.75 & 0. & 300 \\
\hline G64F & $S F \perp A$ & 3.80 & 11.00 & 0.35 & 6.20 & 275 \\
\hline G65F & $S F 15$ & 5.30 & 27.80 & 0.1 .9 & 6.40 & 300 \\
\hline $666 F$ & SFL 6 & $5+30$ & 27.30 & 0.19 & $5+80$ & 300 \\
\hline
\end{tabular}


Zane Hills-Purcell Mountains Area Sediment and Water Samples

\begin{tabular}{|c|c|c|c|c|c|c|c|c|}
\hline $\begin{array}{l}\text { Map } \\
\text { No. }\end{array}$ & Field No. & $\begin{array}{l}U_{\mathrm{PPm}} \\
(\mathrm{RAA})^{1}\end{array}$ & Th ppm & U/Th & $\mathrm{rK}_{2} \mathrm{O}$ & $\begin{array}{l}\text { U Ppm } \\
(L A S L)\end{array}$ & $\begin{array}{l}u \text { ppb } \\
(L A S L)^{l}\end{array}$ & cps \\
\hline & $5 E 3535$ & 0 & 9.00 & 0. & 1.53 & & & \\
\hline$\pi 2$ & $5 E 3505$ & 0.80 & 21.50 & 0.07 & 1,50 & & & \\
\hline 113 & GEXA96 & 2.80 & 16.00 & 0.11 & $1 .+62$ & 5.40 & 0.75 & \\
\hline$\sum 14$ & $5 F 2895$ & 2.00 & 15,50 & 0.13 & 1.42 & & & 100 \\
\hline 밍 & SE2SAS & 7.90 & 17.50 & 0.45 & ?? 3 & $15 \cdot 50$ & 0.65 & \\
\hline 116 & $51: 2938$ & 7.50 & 42.00 & 0.18 & 2.30 & 14.60 & & 200 \\
\hline 17 & $5 E 2915$ & 7.20 & 15.00 & 0.48 & 2.18 & 10.40 & 0.95 & \\
\hline 108 & 51:2926 & 8.20 & 20.00 & 0.41 & 2.33 & 10.10 & & 200 \\
\hline $5 \%$ & $5 E 290 S$ & 10.20 & 44.00 & 0.23 & 2.3 .3 & & 0.75 & \\
\hline 210 & SE2B6S & 8.20 & .15 .00 & 0.55 & 2.00 & 16.00 & 0.60 & 200 \\
\hline $0: 11$ & 912385 & 7.70 & 24.50 & $0.3 \%$ & 1.40 & 14.830 & 0.50 & 100 \\
\hline 112 & $5 E: 2886$ & 6.70 & 50.00 & 0.1 .3 & ป. .56 & 17.20 & 0.75 & \\
\hline$[1,3$ & $5 E 2875$ & 6.60 & 1.1 .50 & 0.97 & 1.46 & 1.1 .1 .0 & 0.70 & \\
\hline C1) A & $5 E 2829$ & 7.90 & -48.50 & 0.1 .6 & 2.07 & 16.10 & & 200 \\
\hline$[15$ & 5E:38.35 & 5.70 & 22.00 & 0.26 & 2.75 & $15 \cdot 1.0$ & & \\
\hline$[11,6$ & $5 E 2845$ & 5.70 & 27.00 & 0.21 & 1.92 & 16.10 & 0.35 & 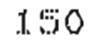 \\
\hline$[117$ & SE2575 & 30.00 & 20.50 & 1.46 & 2.15 & 48.00 & 0.40 & 200 \\
\hline 018 & SE2555 & 11.90 & 17.50 & 0.68 & $\therefore .18$ & $22-00$ & 0.30 & \\
\hline 1119 & 5E256S & 13.30 & 87.00 & 0.23 & $2.0 \Leftrightarrow$ & 38.00 & 0.20 & 1.60 \\
\hline$n 20$ & $5 E 310 S$ & 2.80 & 7.50 & 0.37 & 1.24 & 4.50 & 1.00 & \\
\hline $02 \mathrm{~J}$ & 513095 & 5.30 & 1.2 .00 & 0.44 & 1.73 & 8.50 & 1. $2 ? 5$ & \\
\hline$[122$ & $5 E 3085$ & 2.70 & 12.50 & 0.22 & 2.45 & 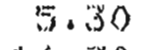 & 0.65 & 150 \\
\hline 1123 & $5 E_{2}^{\prime 2} 86$ & 6.90 & 61.50 & 0.11 & 2.14 & $16 \cdot .30$ & 0.45 & \\
\hline 1124 & $5 F 2595$ & $17 \% .80$ & $18.0\rangle$ & $0.9 \%$ & 1.92 & & 0.65 & \\
\hline $12=5$ & $5 E 2625$ & 4.60 & 1.2 .00 & 0.38 & 1.64 & $7+60$ & 1.65 & \\
\hline 106 & $5 F 2646$ & 7.50 & 20.00 & 0.38 & 1.66 & 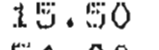 & 0.70 & \\
\hline$\$ 127$ & $5 E 2606$ & 18.00 & $1.8+40$ & 0.98 & 1.75 & 54.00 & 0.85 & 200 \\
\hline 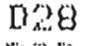 & EE271S & 23.40 & 42.50 & 0.56 & 2.17 & 26.00 & 8.95 & 200 \\
\hline 129 & $\exists=2706$ & 21.30 & 37.00 & 0.158 & 2.18 & 40.00 & 0.70 & 200 \\
\hline 2130 & $5 E 2685$ & 50.10 & 42.00 & 0.12 & 2.62 & 13.80 & 3.26 & 200 \\
\hline עo.3. & $5 F 2695$ & 1.4 .130 & 57.50 & 0.25 & 2.06 & $34+00$ & 1.95 & 200 \\
\hline$[1,32$ & 50263 & 14.90 & 40.50 & 0.37 & 2.51 & $25+00$ & 2.95 & 200 \\
\hline 033 & $5=2698$ & I. . 40 & 7.00 & 0.20 & 0.97 & & 1.70 & \\
\hline D.s. & $5=2665$ & 2.40 & 4.50 & 0.83 & 1.09 & 3.30 & 2.70 & \\
\hline 1036 & $5 E 2639$ & 7.80 & 56.00 & $0 . \mathrm{J} .4$ & 1.55 & $72+90$ & 3.25 & \\
\hline 1136 & $5 E 2645$ & 2.40 & 23.00 & 0.10 & 1.48 & & 2.40 & \\
\hline 137 & $5 E 2745$ & 35.00 & $6 \% .00$ & 0.52 & 2.70 & 60.00 & 1.90 & 700 \\
\hline 01.38 & $5 E 2736$ & 75.90 & $78+00$ & 0.20 & 2.06 & 44.00 & $\therefore .15$ & 300 \\
\hline 0 & 5E2720 & $2+50$ & 7.50 & 0.3 .3 & 1.00 & 3.40 & 0.85 & 100 \\
\hline$H 1$ & $5 E 3395$ & 4.30 & 6.50 & $0.6 c$ & J. 41 & 4.90 & 0.20 & \\
\hline$H_{2}^{2}$ & $5 E 3415$ & 2.90 & 6.50 & 0.45 & 1.06 & 3.00 & 2.30 & 80 \\
\hline $\mathrm{H}=3$ & SE.340S & 2.90 & $12,(50)$ & 0.23 & 1.37 & & 0.35 & 80 \\
\hline HA & $5 F 3489$ & 4.00 & 12.00 & 0.33 & 1.39 & & & 1.00 \\
\hline Hs & $5 E 3425$ & 1.80 & $1.2+50$ & 0.14 & 1.20 & 3.80 & 1.75 & 1.50 \\
\hline$H 6$ & SES3435 & 2.10 & 10.50 & 0.20 & 1.17 & 3.50 & 1.90 & 120 \\
\hline$H 7$ & $5 E-3445$ & 4.30 & 12.50 & 0.34 & 1.40 & 6.00 & 2.40 & 200 \\
\hline
\end{tabular}

). See $\mathrm{p} \cdot \mathrm{A}-2$. 


\begin{tabular}{|c|c|c|c|c|c|c|c|c|}
\hline $\begin{array}{l}\text { Map } \\
\text { No } \\
1 \%\end{array}$ & $\begin{array}{l}\text { Field No. } \\
\text { BE } 3455\end{array}$ & $\begin{array}{l}\text { U ppm } \\
(\mathrm{RAA}) \\
9.60\end{array}$ & $\begin{array}{l}\text { Th ppm } \\
2 x+80\end{array}$ & $\begin{array}{l}\text { U/Th } \\
0.40\end{array}$ & $\begin{array}{l}q \mathrm{~K}_{2} \mathrm{O} \\
1.60\end{array}$ & $\begin{array}{l}U \text { ppm } \\
(L A S L){ }^{1} \\
11: 10.10\end{array}$ & $\begin{array}{l}\text { U ppb } \\
(\text { LASL) }\end{array}$ & 205 \\
\hline$H \%$ & $51: 3468$ & 8.30 & 9.50 & 0.87 & 1.72 & & 2.70 & 200 \\
\hline $\begin{array}{l}\text { H10 } \\
\text { Hi. }\end{array}$ & $\begin{array}{l}3565 \\
53525\end{array}$ & $\begin{array}{l}7.40 \\
6.10\end{array}$ & $\begin{array}{l}19+00 \\
21.00\end{array}$ & $\begin{array}{l}0.39 \\
0.29\end{array}$ & $\begin{array}{l}1.96 \\
2.00\end{array}$ & $\begin{array}{r}\text { 1. } 8.00 \\
9.80\end{array}$ & 0.45 & $\begin{array}{l}200 \\
200\end{array}$ \\
\hline$\omega_{12}$ & 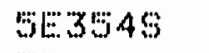 & 6.90 & 42.00 & 0.1 .6 & 2.62 & 1.4 .00 & 1.80 & 200 \\
\hline 413 & SEX 365 & $4+60$ & 23.00 & 0.20 & 2.17 & & 0.20 & 200 \\
\hline$H: 1$ & $8=3065$ & $15 \cdot 60$ & 26.50 & 0.59 & 2.61 & 29.00 & 0.95 & \\
\hline H. & 58.5078 & $13+30$ & 27.00 & 0.49 & 1.32 & $23+00$ & 0.58 & 200 \\
\hline H. 1.6 & $5 E 3055$ & 8.80 & 40.60 & 0.22 & 2.50 & & $1+45$ & \\
\hline H.\% & $5 E 3049$ & 1.0 .70 & 28.100 & 0.38 & 2.72 & 18.00 & 0.50 & \\
\hline HIS & 513000 & $13+20$ & 32.00 & 0.41 & $2.4 \%$ & 28.00 & 1.40 & 200 \\
\hline H.9 & $51: 3016$ & 5.40 & 62.00 & 0.09 & 2.30 & 19.20 & 0.83 & 200 \\
\hline$H 20$ & 563028 & 7.10 & $38 . \cdots 0$ & 0.18 & 1.91 & 13.90 & & 200 \\
\hline 421 & 953035 & 13.90 & 36.00 & 0.1 .7 & $2+18$ & 1.7 .40 & 0.65 & 200 \\
\hline 122 & 562998 & 6.10 & 24.60 & 0.25 & 2.00 & 26.00 & 0.20 & 200 \\
\hline 423 & EE298S & 7.60 & 34.00 & 0.22 & 2.26 & 1.4 .70 & 0.75 & 200 \\
\hline$W^{2}$ & 862969 & 9.70 & 30.60 & 0.32 & 2.50 & 18.10 & 1. .30 & 200 \\
\hline 423 & $9 E 2976$ & 7.60 & 32.00 & 0.24 & 2.49 & 12.00 & $1.3 \%$ & 200 \\
\hline 126 & $1: 2018$ & 6.70 & $3.3+50$ & 0.20 & 1.99 & 16.40 & 0.83 & 300 \\
\hline $12 \%$ & :12956 & 3.80 & 13.00 & 0.29 & $2+0 ?$ & & 0.45 & 200 \\
\hline 128 & 62789 & $9+60$ & $28+00$ & 0.30 & 2.25 & 1.8 .40 & 1.56 & 200 \\
\hline 429 & $61: 2798$ & 12.90 & 100.00 & 0.26 & $2+13$ & 31.00 & 1.80 & 200 \\
\hline 130 & :12778 & 10.90 & 30.30 & 0.36 & 1.94 & $1 \% .70$ & 1.60 & 300 \\
\hline 4.31 & $61: 2768$ & 37.00 & $6 \% .00$ & 0.61 & 2.49 & $77+0$ & 1.05 & 600 \\
\hline 132 & $61: 2798$ & $2+3.00$ & $23+10$ & 1. .06 & 1.63 & 36.0 & $2 \pm 60$ & 600 \\
\hline 133 & 3153335 & 9.90 & 44.50 & 0.22 & $2.6 \%$ & $22+00$ & 0.78 & 500 \\
\hline 134 & 6153316 & 29.00 & 73.00 & 0.40 & $3+24$ & 35.00 & 0.70 & 400 \\
\hline 433 & 963329 & 26.00 & 210.00 & 0.35 & 3.32 & 32.00 & 0.65 & 400 \\
\hline 136 & (1:3345 & $12+90$ & 19.90 & 0.66 & 2.17 & 113.20 & 0.30 & \\
\hline$H 3 \%$ & $91: 3368$ & .6 .90 & 80.00 & 0.21 & 3.08 & $31+00$ & $1 .+80$ & 300 \\
\hline 438 & $31: 3389$ & 30.00 & $120+00$ & 0.25 & $3+30$ & 63.00 & 1.95 & \\
\hline 139 & 5E37\% & $\because 1.00$ & 65.00 & 0.32 & 2.78 & 40.00 & 1.70 & \\
\hline$H 40$ & $5 E 3285$ & 5.30 & $12+00$ & 0.44 & 1.94 & 6.80 & 1. . 50 & 160 \\
\hline$M 4.1$ & 515378 & 3.30 & $13+00$ & 0.41 & 2.37 & 8.40 & 0.75 & 160 \\
\hline$H 42$ & $51: 3236$ & 3.80 & 10.00 & 0.36 & 1.88 & 8.30 & 0.75 & 1.90 \\
\hline $4 \times 3$ & 953265 & 4.50 & 16.00 & 0.28 & 1.98 & 6.70 & 1.00 & 100 \\
\hline 144 & 615329 & 7.00 & 18.60 & 0.38 & 2.74 & $12+80$ & & \\
\hline HAS & $5 E 3309$ & $1 \% .80$ & 21.80 & 0.83 & 2.74 & 23.00 & 0.85 & \\
\hline$H 46$ & 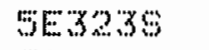 & $\because: 30$ & $1 \%,: 30$ & $0+31$ & $2: 1.6$ & 9.90 & 0.20 & \\
\hline$H A 7$ & 513246 & 4.70 & $1: 3.00$ & $0.3 \%$ & 2.53 & 10.10 & 0.70 & \\
\hline 148 & 583296 & 5.50 & $1.3+60$ & $0.4 \mathrm{~L}$ & 1.90 & 9.90 & 0.90 & 300 \\
\hline H49 & 353218 & 6.60 & $17 \cdot 30$ & 0.38 & $2+23$ & 1.1 .20 & & 300 \\
\hline MOO & GE: 3128 & 2.30 & 13.30 & 0.1 .7 & 1.12 & 4.100 & 1.33 & \\
\hline$H: 1$ & $5 E 31.36$ & $2+50$ & 9.00 & 0.28 & 1.17 & 4.40 & 1.05 & 400 \\
\hline $1+132$ & GE 3165 & $17 \% .00$ & 47.00 & 1.21 & 2.47 & 59.00 & 0.65 & \\
\hline 403 & $B E 3149$ & 9.0 & & & 3.69 & 74.00 & 1.30 & \\
\hline $40 \%$ & $5 E 3165$ & $16 \cdot 10$ & 37.00 & 0.44 & 2.89 & 27.00 & 0.20 & 450 \\
\hline $4: 3$ & $5 E 31 \%$ & $22 \cdot 70$ & 54.00 & 0.42 & $3 \pm 1.4$ & 39.00 & $0.4 \%$ & $4: 30$ \\
\hline $4=66$ & 5E 31.85 & 43.00 & 44.60 & 1.01 & 3.00 & 77.00 & $1.0 \mathrm{~s}$ & 450 \\
\hline $45 \%$ & 583198 & $56+00$ & 64.00 & 0.80 & 2.67 & 59.00 & $1+40$ & 400 \\
\hline
\end{tabular}

1. See p. A-2. 

Zane Hills-Purcell Mountains Area
Rock Samples

\begin{tabular}{|c|c|c|c|c|c|c|}
\hline $\begin{array}{l}\text { Map } \\
\text { No. } \\
\text { all R }\end{array}$ & $\begin{array}{l}\text { Field No. } \\
\text { ziHIA }\end{array}$ & $\begin{array}{r}3 p p m \\
2 p+10\end{array}$ & $\begin{array}{l}\text { Th ppm } \\
80.60)\end{array}$ & $\begin{array}{l}\text { U/Th } \\
0.05\end{array}$ & $\begin{array}{l}8 K_{2} 0 \\
6+31\end{array}$ & $\begin{array}{c}\mathrm{cps} \\
200\end{array}$ \\
\hline IIT & $2 H 13 \mathrm{~A}$ & 1.7 .20 & 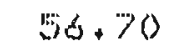 & 0.30 & $6+45$ & 000 \\
\hline$[1 ; x)$ & Znt.3 & 6.80 & 24.50 & 0.28 & 8.1.5 & 000 \\
\hline 1). $4 \mathrm{n}$ & $y .1-1,2$ & 3.60 & 63.00 & 0.06 & 7.15 & 745 \\
\hline II:F & ZHII. A & 8.50 & 1.1 .40 & 0.57 & 4.50 & 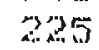 \\
\hline$D \in R$ & Z..H.1. & 1. $+\cdots 0$ & $1.5 \cdot 10$ & 0.10 & 3.37 & 225 \\
\hline Ø̋ッ & $2+100$ & 1.70 & 11.40 & 0.15 & 3.27 & 230 \\
\hline LI8R & $2 H 9$ & 2.50 & 12.00 & 0.21 & 4.03 & 335 \\
\hline 1992 & 2.16 & 4.80 & 44.80 & 0.11 & 2.80 & 275 \\
\hline DIOK & SES\% & I. .80 & 10.30 & $0.1 \%$ & 1. . 68 & \\
\hline II.11F & SE51R & 1.10 & 8.00 & 0.18 & 1.10 & 70 \\
\hline MIR & Z & 1.40 & 9.37 & 0.15 & 0.75 & 95 \\
\hline 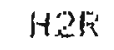 & $Z H_{\text {A. }}$ A A & 3.40 & 1.7 .70 & 0.89 & 4.855 & 300 \\
\hline H3F & ZH2ลAि & 2.30 & 20.30 & 0.1 .1 & 4.44 & 300 \\
\hline $14 \sqrt{2}$ & ZHZ2EII & 49.00 & 1.3 .50 & 3.63 & 6.28 & 300 \\
\hline H5R & ZHZ2EZ & 19.40 & $\therefore 2 \cdot 30$ & 0.91. & 6.1 .5 & 300 \\
\hline HGli & Z H & 3.20 & 1.50 & 0.28 & 2.53 & 235 \\
\hline 州\% & $Z 420$ & 1.60 & 2.08 & 0.77 & 3.14 & $2+0$ \\
\hline$H B E$ & $Z 1 H_{2 O A}$ & 2.80 & 8.85 & 0.32 & 3.21 & 210 \\
\hline HQR & ZH. 17 & 1.60 & 12.50 & 0.1 .3 & 3.16 & 190 \\
\hline H.OR & $2+1.8$ & 1.40 & 9.40 & 0.15 & 3.01 & 230 \\
\hline H.1. I. & $21+17$ & 0.70 & 1. 250 & 0.08 & 2.90 & 210 \\
\hline$H \perp 2 F$ & $2 H .17 \mathrm{~A}$ & 1.20 & 1.1 .00 & 0.11 & $3.2 y$ & 2.20 \\
\hline HJ.3F & ZWH.t. & 0.90 & 1.4 .00 & 0.06 & 2.80 & $2: 10$ \\
\hline$H 14 F^{\circ}$ & 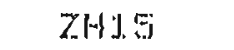 & 1.20 & 24.60 & 0.08 & $2.9 \%$ & 210 \\
\hline HAS & 2747 & 2.50 & 18.30 & $\Delta .13$ & 3.41 & 360 \\
\hline$H 16 \mathrm{~F}$ & ZHE & 2.40 & 13.50 & 0.18 & 3.21 & 250 \\
\hline$H \perp 7 \pi$ & $Z H G A$ & 1.70 & 13.50 & 0.13 & 3.35 & 250 \\
\hline H18R & 2.145 & 0. & $1.4 \cdot 10$ & 0 & 3.82 & 350 \\
\hline HLSF & ZHEA & 2.30 & $7 A+00$ & 0.03 & 5.68 & 500 \\
\hline H2OK & $Z H A A$ & 3.10 & 38.30 & 0.05 & 4.73 & 580 \\
\hline H2」R & $Z M A Y$ & $\because \because, A()$ & $51+00$ & 0.11 & 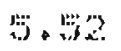 & 580 \\
\hline Hב2fi & ZHACC & 0 & $11 .: 30$ & 0 & 6.1 .3 & 580 \\
\hline H23F & $2 \%$ & 3.70 & 59.90 & 0.06 & 6.65 & 650 \\
\hline H24K & $2 \mathrm{H} 2$ & $\because .40$ & 58.30 & 0.09 & 62 & 650 \\
\hline 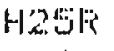 & 가나. & 0.60 & $\therefore .08$ & 0.29 & 0.83 & 475 \\
\hline$H 26 F^{\circ}$ & $\dddot{*} . \cdots \mathrm{A}$ & 8.80 & $5 . .00$ & 0.16 & 6.1 .9 & 1.00 \\
\hline H27א & : & 0 & $1.26,30$ & 0.03 & 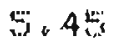 & 900 \\
\hline H2GF & 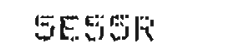 & 6.60 & 21.00 & 0.81 & 3.35 & \\
\hline $\mathrm{H}_{2} 2 \mathrm{Fi}$ & WCos & 9.10 & 26.00 & 0.2. & 4.78 & 400 \\
\hline$W 30 F_{2}$ & WCI. & 18.40 & $31+00$ & () . & 4.49 & 540 \\
\hline $43 \pi$ & $w(C, \bar{c})$ & 8.90 & 18.70 & 0.32 & 3.34 & 380 \\
\hline 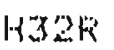 & WCB & 32.70 & 41.60 & 0.79 & $4.5 \%$ & 580 \\
\hline H33 & WCA & 11.90 & 38.30 & 0.31 & 4.67 & 620 \\
\hline$H 34 R$ & $W C 8$ & 15.90 & 35.40 & 0.45 & 1.48 & 620 \\
\hline $435 \mathrm{R}$ & WC5 & 15.50 & 25.00 & 0.62 & 3.833 & 540 \\
\hline W 362 & WC $6 \cdots 1$ & .4 .00 & 30.20 & 0.13 & 4.36 & 480 \\
\hline 州 378 & WC $6 \ldots 2$ & .10 .40 & 20.90 & 0.070 & 4.80 & 480 \\
\hline H386 & WC 7 & 9.00 & 35.40 & 0.210 & 3.1 .88 & 300 \\
\hline
\end{tabular}


Copper River Basin

Sediment and Water Samples

\begin{tabular}{|c|c|c|c|c|c|c|c|c|}
\hline C. & $5 E 3665$ & 1.40 & 10.00 & 0.14 & 1.20 & & & \\
\hline CE & EE:365S & 1.30 & 7.00 & 0.1 .9 & d. . 32 & $\begin{array}{l}0.50 \\
0.70\end{array}$ & 0.75 & \\
\hline 0.3 & $5 E 3645$ & 2.00 & 11.50 & 0.1 .7 & 1.34 & 2.70 & & 80 \\
\hline$C<4$ & $5 E 3635$ & 1.80 & 14.50 & 0.12 & $\underline{1}+\underline{3}$ & 2.40 & 0.85 & \\
\hline $\mathrm{CS}$ & $5 E 3625$ & 2.50 & 8.00 & 0.31 & $\sqrt{1} .20$ & 2.20 & 1.30 & 70 \\
\hline 60 & $5 E 3679$ & 1.40 & 6.20 & 0.23 & 1.81 & 3.00 & 1.75 & \\
\hline K.I & $5 E 3805$ & 2.00 & 5.50 & 0.36 & 1.02 & 1.90 & 0.40 & 60 \\
\hline k゙?? & $5 E 379 S$ & 1.70 & 7.90 & 0.23 & 1.13 & 2.60 & 0.35 & 60 \\
\hline$k .3$ & $5 E .3793$ & 2.50 & 5.70 & 0.44 & 0.84 & 2.50 & 1.10 & 40 \\
\hline バィ & $5 E 3779$ & 2.00 & 5.50 & 0.36 & 0.88 & 2.50 & 0.70 & 40 \\
\hline$k: \therefore$ & BMㄴㅇㅛ & 0.50 & 1.20 & 0.42 & 1.50 & 1.20 & 1.00 & 45 \\
\hline kib & 5M․․ & 0. & 2.75 & 0 & 1.00 & 2.20 & 1.00 & 45 \\
\hline $\mathfrak{n}$ & $5 M 279$ & 0 & 3.75 & 0. & 1.4 .3 & 1.20 & 0.35 & 45 \\
\hline バß & Б以295 & 1.60 & 5.75 & 0.28 & 1.15 & & 0.85 & 28 \\
\hline ハ! & 5M285 & 0. & 16.00 & 0. & 1.40 & & $.2 \cdot 1.5$ & 28 \\
\hline K10 & シハレフ9 & 3.830 & 3.80 & 0.74 & 0.75 & 3.30 & & 38 \\
\hline 《11. & SM20S & i. .10 & 4.25 & 0.26 & 0.71 & 2.30 & 0.90 & 43 \\
\hline バす? & らバレタ & 2.10 & 4.00 & 0.53 & 0.89 & 1.00 & 0.60 & 38 \\
\hline K113 & $5 K 1165$ & 1.90 & 3.30 & 0.58 & 0.75 & 1.80 & 3.25 & 38 \\
\hline 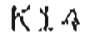 & WM22S & 0. & 3.75 & 0 & 0.44 & & 1.00 & 35 \\
\hline K!: & $5 k 136$ & 0.80 & 2,30 & 0.35 & 0.56 & 1.30 & 1.55 & 38 \\
\hline k.16 & 5249 & 0. & 3.00 & 0 & 0.5 .3 & & & 25 \\
\hline K\\
& $5 M 216$ & 1.00 & 3.00 & $0+33$ & 0.62 & & & 35 \\
\hline$k 13$ & 淤ハ28 & 0. & 2.80 & 0 & 0.99 & 1.30 & J. . 55 & $40)$ \\
\hline$K 1 \%$ & ت゙バ1 & 1. . 40 & 2.80 & 0.50 & 0.76 & 1.00 & 3. . 40 & 20 \\
\hline$K 20$ & 涪バ1.5 & 1.00 & .1. . 80 & 0.56 & 0.75 & 0.69 & 0.75 & 30 \\
\hline 1221 & ऊ人ys & .1. . 00 & 3.30 & 0.30 & 0.80 & i. .40 & 0.90 & 09 \\
\hline 32 & $5 k 85$ & 0.40 & 13.80 & 0.07 & 0.92 & 1.60 & 0.75 & 40 \\
\hline 523 & 范バ75 & $\underline{2} \div \underline{\underline{Q}}$ & 3.80 & 0.63 & $0.8 \mathrm{y}$ & 1.80 & 0.65 & 40 \\
\hline K๐. & 0.2209 & 0.80 & 6.74 & 0.12 & 0.77 & & & 190 \\
\hline K2\% & $5 C 2215$ & 0. & 3.49 & 0 & 0.73 & & & 35 \\
\hline$k 2$ & $5 E 3>65$ & 2.50 & 2.70 & 0.93 & 0.95 & 1.60 & & 10 \\
\hline אי\% & BE- 375 & 1.30 & 6.00 & 0.22 & 0.77 & 1.80 & $0.4 \mathrm{~J}$ & 45 \\
\hline KरB & $B E 3>43$ & 1.60 & 2.70 & 0.59 & 0.80 & 1.60 & 1.05 & 50 \\
\hline K2\% & EE3739 & 1.30 & 4.20 & 0.21 & .1 .10 & 1. .20 & 1.30 & 50 \\
\hline ベ30 & $5 \times 5.3$ & 0 & 0.50 & 0. & 0.99 & 0.90 & 0.70 & 40 \\
\hline 《゙ろむ & $5 K 65$ & 0.40 & 0.0 & 0.80 & 0.87 & 0.51 & 1.55 & 42 \\
\hline 132 & $5 E 3>15$ & 0.30 & 6.20 & 0.05 & 1.13 & 1. .30 & 0.80 & 60 \\
\hline 1533 & EE3725 & 1.00 & 5.70 & 0.18 & 1.24 & 1.90 & 0.60 & 60 \\
\hline 13.34 & $5 M 29 S$ & 0. & 5.50 & 0. & 0.47 & 0.23 & 0.50 & 25 \\
\hline א゙3. & $5 M 185$ & 0. & 4.50 & 0. & 0.40 & 0.23 & 0.25 & 25 \\
\hline 1536 & SMIOS & 0. & 8.25 & 0. & 0.14 & 0.31 & 0.55 & 30 \\
\hline K゙は; & GMU 76 & 0. & 2.75 & 0. & $0.7 \mathrm{l}$ & & 0.70 & 30 \\
\hline 133 & $5 M 15 S$ & 0. & 0.60 & 0 & 0.83 & 0.1 .5 & 0.515 & 30 \\
\hline $13 \%$ & $\operatorname{Sin\perp 4S}$ & 0. & 7.50 & 0. & 0.82 & 0.40 & 1.00 & 30 \\
\hline KAO & GMLOS & 0.80 & 3.50 & 0.23 & 1.09 & 0.47 & $0.79 !: 3$ & 40 \\
\hline
\end{tabular}

1. See p. A-2. 


\begin{tabular}{|c|c|c|c|c|c|c|c|c|}
\hline $\begin{array}{l}\text { Map } \\
\text { No: } \\
\text { FiAl }\end{array}$ & $\begin{array}{l}\text { Field No. } \\
\text { smiss }\end{array}$ & $\begin{array}{l}\text { U PPm } \\
(R A A) \\
0 !\end{array}$ & Th Tpom $^{\circ}$ & U/Th & $\% 620$ & $\begin{array}{l}U \text { ppm } \\
(1, A s\})^{\prime} \\
0.25\end{array}$ & $\begin{array}{c}U p p b \\
(L A S L) \\
0^{(L S S L}\end{array}$ & .50 \\
\hline$K A 2$ & 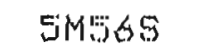 & $1 . .60$ & 2.10 & 0.76 & 1.00 & 1.36 & J. 35 & 40 \\
\hline 1143 & $5 M 605$ & 1.10 & 2.79 & 0.40 & 1.12 & 0.90 & 1.40 & \\
\hline 1゙タ4 & जM6:5 & 1.20 & 4.50 & 0.27 & 0.80 & $1 .+10$ & 1. . 30 & \\
\hline KAs & : & $\mathrm{l} .40$ & 2.78 & 0.51 & 0.90 & 0.93 & 1.55 & \\
\hline KAS & $5 M G 4 B$ & 1.50 & 35 & 0.28 & 0.88 & 1. .80 & & 42 \\
\hline KA\% & $5 k 3 ! S$ & 0.80 & 4.30 & 0.199 & 0.923 & 1.80 & 0.40 & 42 \\
\hline K16 & 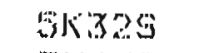 & 1.00 & 3.30 & 0.30 & 0.80 & 1.40 & 0.85 & 42 \\
\hline バタ & 54635 & 1.60 & 4.50 & 0.33 & 0.90 & 2.10 & & 42 \\
\hline k゚E? & $5 M 625$ & 0 & 3.50 & 0 & 0.888 & 1.60 & & 40 \\
\hline א゙5: & $5 M G \perp 5$ & (). & 3.76 & (). & 0.88 & 1.60 & & 14 \\
\hline バョ? & 5ドュ日S & 0 & 2.50 & 0. & 0.983 & 1. 40 & 1.10 & \\
\hline$k 103$ & $5 \times 2255$ & 0.70 & $2+30$ & 0.25 & 0.76 & 1.20 & 1.40 & \\
\hline Ǩ‥? & 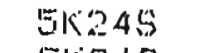 & 0.40 & 6.00 & 0.07 & 0.75 & & & \\
\hline 155 & ت⽖ㄹ6S & 2.10 & 18.30 & 0.45 & 0.73 & . .50 & 0.95 & \\
\hline 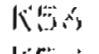 & 5バ275 & 1.10 & 4.00 & 0.28 & $0.5 \%$ & 0.86 & & \\
\hline$k 6$ & 51298 & 0.60 & 3.00 & 0.20 & 0.83 & 1. .30 & 1.20 & \\
\hline kib & 5M도 & 1.20 & 1. .45 & 0.83 & 11.10 & 3.20 & 1.10 & 10 \\
\hline 16 & 54605 & 0 & 2.55 & 0. & 1.31. & 1.30 & 0.55 & 10 \\
\hline Kڤ। & : & cis & 250 & 0 & 0.90 & 0.9 .4 & 0.55 & 40 \\
\hline$k 6:$ & SM:1\%S & 0.830 & 3.50 & 0.23 & $1 .+03$ & $1+20$ & & 10 \\
\hline Ks? & 要的心 & 1.20 & 5.20 & 0.23 & 1.1 .3 & 0.910 & 1.45 & 40 \\
\hline 563 & $5 M 5 S$ & 1.20 & 4.50 & 0.27 & 0.80 & 1.30 & 0.70 & 40 \\
\hline$k b \cdot$ & $5 M 45$ & 1.20 & 3.313 & 0.37 & 0.82 & 1.70 & $0.4 \mathrm{~s}$ & 36 \\
\hline 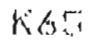 & $5 M 8 S$ & $1.2>0$ & 2.25 & 0.53 & 1.00 & 1.40 & 1.10 & $3 ?$ \\
\hline バにட & 5以> & 1.30 & 3.00 & 0.40 & 0.94 & 1.80 & 0.65 & 32 \\
\hline Ko: & SM9S & 0.90 & 200 & 0.36 & 0.86 & 1.60 & 1.15 & 36 \\
\hline r.6. & $5 \times 33$ & 0.70 & 9.00 & 0.11 & 1.18 & 1. .40 & 1.40 & 48 \\
\hline バ6す & БК2 & 1.30 & 2.30 & 0.57 & 1.180 & 1.20 & 0.75 & 56 \\
\hline 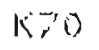 & $5 E 3685$ & 1. 140 & 6.00 & 0.23 & $0.8 \%$ & 1.30 & & \\
\hline ハ?! & WMIIS & 1.30 & 1.: & 0.24 & $0.8\}$ & 1.80 & 0.40 & \\
\hline $14 \% ?$ & SE3709 & 2.10 & 12.00 & $0.1 \%$ & 1. .24 & 2.10 & & 60 \\
\hline$k \mathfrak{y}$ & $G E 3695$ & 2.00 & 13.60 & 0.36 & 1.20 & 2.00 & & 60 \\
\hline$k \%$ & 54525 & 1.1 .0 & 5.50 & 0.20 & 1.07 & 1.50 & 0.50 & 30 \\
\hline א゙フ & $6 M 549$ & 0. & 6.50 & 0 & $\stackrel{2.00}{=}$ & 1.90 & & 56 \\
\hline$k 76$ & $5 M 535$ & 0.70 & 5.00 & 0.14 & 9.29 & 1.80 & & 32 \\
\hline バタ: & $5 M 505$ & .1 .00 & 3.00 & 0.3 .3 & 1.36 & 1.630 & 0.45 & 36 \\
\hline 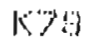 & $5 M 515$ & 0. & 2.50 & 0. & 1.31 & 1.60 & 0.55 & 36 \\
\hline 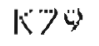 & $5 M 55 S$ & 0.50 & 2.50 & 0.20 & $0+20$ & $0.5 ?$ & 0.75 & 20 \\
\hline 1380 & $5 M 49 S$ & 2.00 & 2.50 & 0.80 & 1.30 & 1.70 & 0.45 & 50 \\
\hline$k 8 \%$ & $5 M 485$ & 0. & 6.50 & 0 & 1.40 & 1.60 & 0.55 & 50 \\
\hline $\mathfrak{k} ; 3:$ & 吠238 & 0.50 & 2000 & 0.25 & 1.33 & 0.70 & 1.95 & 45 \\
\hline$k \& 33$ & WK22G & 1.30 & 2.80 & 0.46 & 1.38 & & & 45 \\
\hline 1831 & تパ20S & .1 .40 & 2.00 & 0.70 & 1.32 & 0.663 & 1.40 & 45 \\
\hline KB! & 5ド2\S & 0. & 1.530 & 0 & 1.40 & & & 45 \\
\hline 1486 & $5 M 695$ & 0.80 & 1.25 & 0.61 & 0.39 & 0.69 & 0.45 & 50 \\
\hline 1587 & $5 M 695$ & 1.60 & 4.00 & 0.40 & 0.40 & 1.50 & 0.35 & 50 \\
\hline 1584 & $5 M 705$ & 0.80 & 2.73 & 0.29 & 0.72 & 0.38 & 1.90 & 45 \\
\hline K8! & $5 M " J 9$ & 0 & 1.75 & 0 & 0.63 & $0.8 \%$ & 0.70 & 80 \\
\hline 1550 & GM7.S & 1.20 & 1.75 & 0.69 & 0.720 & 0.69 & 1.40 & 30 \\
\hline
\end{tabular}

1. See p. A-2. 


\begin{tabular}{|c|c|c|c|c|c|c|}
\hline $\begin{array}{l}\text { Map } \\
\text { io. }\end{array}$ & Field No. & (I) $\mathrm{n} \rho \mathrm{m}$ & Th ppm & ג & $\because \mathrm{K}_{2} \mathrm{O}$ & cps \\
\hline$\because F 1$ & $\because E \perp 6$ & 0.50 & J. 1.50 & $0.0 \mathrm{~A}$ & 3.18 & \\
\hline$\therefore F$ & 5E2S & 0.50 & 11.70 & 0.0 A & 1.28 & \\
\hline$+1=3$ & $5 E 35$ & 1.00 & 19.90 & 0.05 & 3.65 & 70 \\
\hline$\div F A$ & SEAS & & 21.2 & & 3.82 & 50 \\
\hline 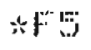 & SESS & & 12.8 & & 2.91 & 8 \\
\hline$\therefore F \&$ & SE马 & & 14.5 & & & \\
\hline$x F=\cdots$ & $\because E .3615$ & 1.30 & 5.00 & 0.26 & 0.48 & \\
\hline$\therefore F B$ & $5 F 3595$ & 1.50 & .13 .00 & 0.1 .2 & 0.51 & \\
\hline$* F$ & $35: 3609$ & 1.00 & 7.50 & 0.13 & 0.80 & \\
\hline
\end{tabular}

\section{Copper River Basin \\ Rock Samples}

\begin{tabular}{|c|c|c|c|c|c|c|}
\hline \multicolumn{7}{|l|}{ Map } \\
\hline $\begin{array}{l}\text { No. } \\
\text { Sin }\end{array}$ & $\begin{array}{l}\text { Fiald No. } \\
\text { GlEGRA }\end{array}$ & $\begin{array}{l}\text { U ppm } \\
1 .+1.0\end{array}$ & $\begin{array}{l}\text { Th pom } \\
26.00\end{array}$ & $\begin{array}{l}\text { U/Th } \\
0.0 .4\end{array}$ & $\begin{array}{l}\mathrm{sk}_{2} \mathrm{O} \\
0.95\end{array}$ & $\begin{array}{l}c p s \\
4: 5\end{array}$ \\
\hline $\cos 26$ & 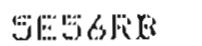 & 0.40 & 3.80 & (). . 1. & 1.40 & 45 \\
\hline KIA & VIECG & 0. & 7.30 & 0 & 20 & 1.00 \\
\hline バさR & 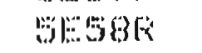 & 0 & 6.50 & 0 & 0.53 & \\
\hline K.SF & 5バリー & 1.10 & 0.10 & I. I. . ০૦> & 0.46 & 38 \\
\hline KKAR & ENֻ1 & 0.90 & 0.30 & 3.00 & 1.10 & \\
\hline KGR & MUE & 0 & 0.10 & 0 & 0.50 & \\
\hline K゙G下 & 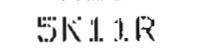 & 0. & 0.20 & 0. & 0.2 .4 & 20 \\
\hline К\%棌 & 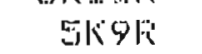 & 0.0 & 0.0 & & 0.20 & 40 \\
\hline Kar & $5 E S>F i$ & 0.10 & 0.10 & 4.00 & 0.40 & 3 \\
\hline パ. & БК々 & 0. & 0.20 & $\Delta$ & 1.56 & 38 \\
\hline K⿺辶⿸ & SESßF & 0.40 & 0.70 & 0.507 & 0.97 & \\
\hline バリ & $5 M 6 F$ & 0.60 & 3.50 & 0.17 & 2.26 & 40 \\
\hline א1 & $5 M A K$ & 1. 00 & 2.00 & 0.60 & J. . 68 & AO) \\
\hline Кз3њ & SМмб8\% & 0. & $\therefore .00$ & 0 & 1.2 .26 & 50 \\
\hline 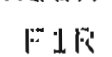 & SESHA & J. .00 & 0.70 & 1.43 & 0.38 & \\
\hline Fin & 51:-5FR & 1.00 & 2.30 & 0.43 & 0.53 & \\
\hline
\end{tabular}

*Sample mean and standard deviation of samples collected in Mt. Hayes quadrangle based on data from that quadrangle only. 
Chitina Valley Area

Sediment and Water Samples

\begin{tabular}{|c|c|c|c|c|c|c|c|c|}
\hline $\begin{array}{l}\text { Map } \\
\text { ivo. } \\
\text { li: } 1\end{array}$ & $\begin{array}{l}\text { Field wo. } \\
\text { sc306s }\end{array}$ & $\begin{array}{l}\text { U } \mathrm{ppm} \\
(\mathrm{RAA}) \\
0.30\end{array}$ & $\begin{array}{l}\text { Th ppm } \\
4.74\end{array}$ & $\begin{array}{l}\mathrm{U} / \mathrm{Th} \\
0.06\end{array}$ & $\begin{array}{l}3 \mathrm{~K}_{2} \mathrm{O} \\
1.46\end{array}$ & $\begin{array}{l}\text { U ppm } \\
(L A S L) ! \\
\therefore . A O\end{array}$ & $\begin{array}{l}\left.U^{(P p b}\right)^{\prime} \\
(L A S L))^{\prime} \\
0.10\end{array}$ & cps \\
\hline$E 2$ & $60 \% 0 \%$ & 0 & $6+7 A$ & 0 & 0.86 & 0.66 & 0.90 & \\
\hline$=3$ & 803218 & 0.60 & 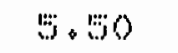 & 0.1 .1 & 1.48 & 2.10 & 0.60 & \\
\hline $1: 4$ & 503083 & 0.30 & 3.24 & 0.06 & 1.32 & 1.10 & 0.90 & \\
\hline $1:: 3$ & 503098 & 0 & 2.99 & 0 & 0.70 & $0.4 \%$ & 0.35 & \\
\hline $1: 66$ & 503279 & 0. & 5.00 & 0 & 0.44 & & 1.00 & \\
\hline $1: \cdots \%$ & $: 06288$ & 0 & 3.26 & 0. & 0.100 & & 0.90 & \\
\hline 18 & 003109 & 0. & 4.74 & 0. & 1.36 & 1.20 & 0.49 & \\
\hline $1: 9$ & $50 \% 1.18$ & 0 & 1050 & 0 & 1.20 & 0.46 & 0.60 & \\
\hline $1: 10$ & 503249 & 0.60 & 3.713 & 0.1 .0 & 0.96 & $1 . .90$ & 0.60 & \\
\hline 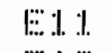 & 50.3138 & 0.70 & 2.35 & 0.30 & 1.20 & 1.00 & 0.70 & \\
\hline$E 12$ & 803126 & 0 & $\%+23$ & 0 & 1.20 & 1.00 & 1.23 & \\
\hline$E 13$ & 60.78 & 0.80 & 5.00 & 0.1 .6 & 0.79 & 2.60 & 0.95 & \\
\hline E.: 4 & 80.8168 & 1.60 & $1 \% .00$ & 0.32 & 0.96 & 4.10 & 1.40 & \\
\hline Fi: $1: 3$ & 503158 & 0 & $11+30$ & 0 & 1.18 & 1.00 & $1+16$ & \\
\hline$E 1.6$ & 30.3149 & 0.80 & 3.60 & 0.23 & $0.5 \%$ & 1.70 & 0.60 & \\
\hline$E 1 \%$ & 906018 & 1.20 & 6.74 & 0.18 & 0.95 & 1.90 & 1.10 & \\
\hline ENo & 50.3028 & 0.70 & 6.99 & 0.10 & 1.00 & & $0 .: 1: 0$ & \\
\hline$E 1 \%$ & sMl. 309 & 0.70 & 3.50 & 0.20 & 1.20 & 2.20 & & \\
\hline 120 & $\sin 318$ & 1.30 & 3.60 & $0.3 \%$ & 1.30 & 2.20 & 0.20 & \\
\hline$E 21$ & $5 M 1328$ & 1.60 & $1 .+00$ & 1.60 & 1.0 .3 & $\therefore 40$ & & \\
\hline $1: 22$ & 641335 & $1+60$ & $4.7 \%$ & 0.33 & 1.64 & 1.70 & & \\
\hline 123 & $3 M 1368$ & 1.90 & $2+25$ & 0.84 & 1.40 & $2: 20$ & 0.20 & \\
\hline 124 & $M M 1345$ & 0.80 & $3+29$ & 0.25 & 1.27 & $2+00$ & $0+30$ & \\
\hline$E 20$ & $5 M 135 s$ & 2.10 & $3+25$ & 0.63 & 1.29 & 1.90 & & \\
\hline $1: 26$ & :MII3\% & 1.90 & 2.79 & 0.65 & 1.62 & 2.20 & & \\
\hline$E 2 \%$ & 1303005 & 0. & 4.49 & 0 & 1.32 & 1.70 & 1.09 & \\
\hline $1: 28$ & $6 \mathrm{M} 1418$ & 2.70 & 4.60 & 0.60 & $1.4 \%$ & $2+60$ & 0.75 & \\
\hline 129 & $3 M 1428$ & $2+70$ & $3+20$ & $0 . \cdots$ & 1. +29 & 3.20 & 1.79 & \\
\hline $1: 30$ & GM A 435 & 1.60 & 3.60 & 0.43 & 1.36 & 3.00 & 0.75 & \\
\hline $\mid: 31$ & GM A56 & 1.60 & 4.25 & 0.35 & 1.40 & 2.60 & 0.40 & \\
\hline 132 & $5 M H O S$ & $2+40$ & 2.150 & 0.96 & $1.0 ?$ & 2.20 & 0.60 & \\
\hline$E 33$ & WMA 478 & 0 & $2+00$ & 0 & 210 & 2.10 & $0.4 \%$ & \\
\hline$E 34$ & Wivt 466 & 0 & 1.50 & 0 & 1.70 & $2+10$ & 0.130 & \\
\hline $\begin{array}{l}136 \\
186\end{array}$ & $\begin{array}{l}50,3048 \\
5 M 1485\end{array}$ & $\begin{array}{l}1.20 \\
0.90\end{array}$ & $\begin{array}{l}6.24 \\
2+75\end{array}$ & 0.19 & $1+40$ & 2.60 & 0.10 & \\
\hline 1337 & 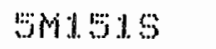 & 0.90 & 4.25 & $\begin{array}{l}0+33 \\
0.21\end{array}$ & $\begin{array}{l}1.0 .59 \\
1.39\end{array}$ & $\begin{array}{l}2.40 \\
3.00\end{array}$ & $\frac{x+10}{0.70}$ & \\
\hline $1: 36$ & MM 1509 & 0.60 & 3.00 & $0.1 \%$ & 1.20 & 1.30 & 0.20 & \\
\hline 639 & "6k69s & 0 & $3: 30$ & 0 & 1.16 & 1.80 & & 70 \\
\hline $1: \div 40$ & $\because: 1 \% 0$ & 0 & 0.80 & 0 & 1.14 & $1+80$ & & 60 \\
\hline E*41 & GMJ 060 & 1.30 & 4.00 & 0.33 & 1.69 & $\therefore 40$ & & \\
\hline E42? & SMIO\% & $0.1: 0$ & $4.2 \%$ & 0.12 & 1. . 49 & 2.00 & 0.83 & \\
\hline$E 43$ & sin. 088 & $1+60$ & 4.00 & 0.40 & 1.33 & 2.40 & & \\
\hline$\because: 44$ & $8=3839$ & 1.80 & 1.0 .70 & 0.1 .7 & 1.40 & $2+40$ & & 100 \\
\hline 1048 & GMIOS9 & $1+10$ & 6.79 & 0.16 & 1.60 & $2+80$ & & \\
\hline$\because 46$ & $5 M I 106$ & 0 & $3+29$ & 0 & 1.53 & 2.40 & 0.50 & \\
\hline$\because 47$ & GMIIS & 1.70 & 4.00 & 0.43 & 1. . 68 & 2.50 & 0.30 & \\
\hline$E .96$ & SMM II IO & 1.60 & $4.7: 3$ & 0.34 & $1.8 \%$ & 2.60 & $3+30$ & \\
\hline$\because 49$ & EMI J & 1.50 & 10.30 & $0.1 .1 \%$ & 1.69 & 1.16 & 1.35 & \\
\hline 1050 & sim 139 & 1.60 & 4.75 & 0.34 & .1 .93 & $2+30$ & & \\
\hline$E \because$ & WMIISS & $1.1: 30$ & 1.1 .00 & 0.1 .3 & 1.46 & 2.00 & 1.10 & \\
\hline
\end{tabular}

1. See p. A-2. 


\begin{tabular}{|c|c|c|c|c|c|c|c|c|}
\hline $\begin{array}{l}\text { Map } \\
\text { No. } \\
\text { Ei: }\end{array}$ & $\begin{array}{l}\text { Field No. } \\
5 M 1165\end{array}$ & $\begin{array}{l}\text { U ppm } \\
(\text { RAA) } \\
2.20 \\
2.20\end{array}$ & $\begin{array}{l}\text { Th ppm } \\
8.29\end{array}$ & $\begin{array}{l}\text { U/Th } \\
0.27\end{array}$ & $\begin{array}{l}\% \mathrm{~K}_{2} \mathrm{O} \\
\mathrm{L}\end{array}$ & $\begin{array}{l}\text { U ppm } \\
\text { (LASL) } \\
3.00\end{array}$ & $\begin{array}{l}\text { U ppb } \\
(\text { LASL) } \\
2.75\end{array}$ & cps \\
\hline $10: 33$ & BM1 I. & 1.50 & 6.24 & 0.24 & 1.31 & 2.60 & & \\
\hline $1 E 54$ & :ML2!:6 & 2.1 .0 & 4.73 & 0.44 & 1.70 & $2 .: 10$ & & \\
\hline 1565 & WMI2AS & 1. .60 & 6.23 & 0.24 & $1.0 .5 \%$ & 2.60 & & \\
\hline $1=56$ & MMIN8S & 1.1 .0 & $2.71: 3$ & 0.40 & 1.62 & 3.00 & & \\
\hline 157 & BM129s & 2.00 & $\because: 00$ & 0.40 & $1 .+65$ & 2.90 & & \\
\hline$E B 8$ & 015616 & 0. & 2.30 & 0 & 1. .67 & $2+90$ & & 10 \\
\hline 1099 & GM1 238 & 2.20 & 8.25 & 0.27 & 1.59 & 2.90 & & \\
\hline$E 60$ & अMI25 & $1 . .50$ & 6.75 & 0.22 & 1.71 & 2.90 & & \\
\hline$E s 1$ & $3 M 1215$ & 2.00 & 8.00 & 0.25 & 1.3 .3 & $2+40$ & & \\
\hline 1362 & WMII. 99 & 1.0 & 1.1 .00 & 0.1 .4 & 1.88 & 3.40 & & \\
\hline $1: 63$ & 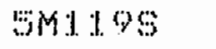 & 2.40 & 9.218 & 0.29 & 1.80 & 2.90 & & \\
\hline$E 64$ & WM1 $20 s$ & 2.00 & 8.00 & 0.23 & 1.29 & 3.00 & & \\
\hline $1 \ldots$ & w4048 & 1.20 & 3.79 & 0.21 & 1.20 & 1.40 & 0.40 & \\
\hline $1 \ldots 2$ & GM103s & 1.1 .0 & 2.00 & 0.56 & $1 .+45$ & 1.60 & 0.20 & \\
\hline $1 \ldots 3$ & W1015 & 0 & 3.00 & 0 & 1.31 & 1.40 & 1.39 & \\
\hline $1 . .9$ & $61: 3919$ & 1.80 & 6.20 & 0.29 & 1.32 & 1.40 & $i .10$ & 10 \\
\hline 1.6 & 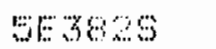 & 2.10 & 3.20 & 0.40 & 1.31 & 1.60 & & 50 \\
\hline $1 \ldots 6$ & MMlO2s & 0 & 3.60 & 0 & $1 . .60$ & 1.50 & 0.38 & \\
\hline $1 . . \%$ & WMOMO & 0 & 1.00 & 0 & 1. 43 & 1..$\because \because 0$ & $1 .+150$ & \\
\hline 10 & 603185 & 0.80 & 4.79 & $0.1 \%$ & 1. .20 & 1.10 & 1.80 & \\
\hline 1.9 & 60398 & 0 & 10.213 & 0 & 1.20 & 1.20 & $1 \cdot 1.5$ & \\
\hline $1 . .10$ & $31: 3848$ & 1.40 & 6.49 & 0.22 & 1.02 & 3.20 & & \\
\hline 1... I. & WM & 1.70 & 2.00 & 0.60 & .00 & 1.90 & 0.40 & 10 \\
\hline 1.12 & WNas & 1.60 & 3.30 & 0.43 & 1.02 & 1.90 & & $4 \%$ \\
\hline $1 \ldots .1 \%$ & 641208 & 1.60 & $: 30$ & 0.30 & $1.0 \%$ & 1.30 & 0.75 & 100 \\
\hline $1 . .14$ & 603208 & 0.80 & $3.7 \%$ & $0.2 \%$ & 1.22 & 1.10 & 1.10 & \\
\hline 1.19 & 603218 & 0.60 & 4.50 & 0.13 & 1.19 & $1+20$ & 1.30 & \\
\hline $1 . .16$ & 60,3226 & 0 & $4.7 \%$ & 0 & $1 . .01$ & 1.10 & 0.90 & \\
\hline $1 \ldots 1 \%$ & 603298 & 0 & 7.213 & 0 & 1.40 & 1.40 & 1.60 & 50 \\
\hline 1...1. & 603308 & 0.70 & 3.50 & 0.20 & 1.12 & 1.40 & 0.60 & \\
\hline $1 . .1 . \%$ & 60.318 & 0.70 & $3+75$ & 0.12 & 0.83 & 1.20 & 1.013 & \\
\hline 1.20 & 303345 & 1.20 & 2.93 & 0.41 & $0.9 \%$ & 1.90 & 1.10 & 30 \\
\hline $1 . .21$ & 60335 & 1.20 & 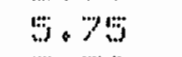 & 0.21 & 1.07 & 3.40 & 0.45 & .60 \\
\hline $1 \ldots 2 \%$ & "вкоо & 3.40 & $8+30$ & 0.64 & 1.06 & 4.00 & 1.50 & $6 \%$ \\
\hline 1.23 & 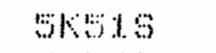 & 1.10 & 6.50 & $0.1 \%$ & 1.22 & 3.20 & $1+60$ & 100 \\
\hline $1 \ldots 24$ & : & $3+30$ & 7.30 & 0.48 & 1.49 & $3+80$ & 0.80 & 60 \\
\hline 1...20 & WMAO & 0 & $2+50$ & 0 & 0.70 & 1.30 & 0.30 & \\
\hline 1.26 & 39468 & $0:$ & 3.79 & 0 & 0.80 & 1.60 & 0.46 & \\
\hline $1 \ldots 2 \%$ & WMA 0 & 0.90 & 3.40 & 0.1 .3 & 1.09 & $2+20$ & 0.130 & $6 \%$ \\
\hline 1.28 & 9438 & $3+20$ & 1.50 & 2.13 & $0.3 \%$ & 6.30 & 0.28 & \\
\hline 1.29 & GM39s & 0.70 & 4.23 & 0.16 & 1.1 .9 & $2+50$ & 0.28 & $4: 3$ \\
\hline 1.30 & 9436 & 0.60 & 2.73 & 0.22 & 1.20 & 1.20 & & 46 \\
\hline $1 \ldots 1$ & GM $3 \%$ & 0.90 & 80 & 0.09 & 1.1 .4 & 2.00 & 0.75 & Bi: \\
\hline $1 \ldots 32$ & WMas & 1.20 & 2.30 & 0.32 & 0.86 & $3+40$ & 0.20 & $49: 3$ \\
\hline $1 \ldots 33$ & WMAS & 1.00 & $3.0 \%$ & 0.33 & 0.74 & 2.40 & 0.20 & 45 \\
\hline 1.3 .4 & WA3S & 1.60 & 3.213 & 0.46 & 0.83 & 1.80 & 0.40 & $4 \%$ \\
\hline 1.36 & 67446 & 0 & 4.25 & 0 & 1.48 & 1.50 & & 43 \\
\hline $1 . .36$ & MM30s & 0.60 & 4.60 & 0.1 .3 & $1 .+0 \%$ & 1.70 & 0.85 & 40 \\
\hline $1.3 \%$ & $\operatorname{mox} 6$ & 0 & 3.25 & 0. & 0.71 & 1.90 & & 30 \\
\hline
\end{tabular}

1. See p. A-2. 


\begin{tabular}{|c|c|c|c|c|c|c|c|c|}
\hline $\begin{array}{l}\text { Map } \\
\mathrm{N} n \\
1.39\end{array}$ & $\begin{array}{l}\text { Field } \mathrm{Nn} \text {. } \\
\text { wimbls }\end{array}$ & $\begin{array}{l}\text { U PPM } \\
\left.(R \Delta \Delta)^{\prime}\right) \\
1 .+800\end{array}$ & $\begin{array}{l}\text { Th } \\
\text { ll. } 45^{n}\end{array}$ & $\begin{array}{l}11 / T h \\
1.24\end{array}$ & 8.400 & 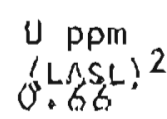 & $\begin{array}{l}U p p b \\
(L \Lambda S I)^{2}\end{array}$ & Gps \\
\hline $1 \ldots 39$ & 61925 & 0.60 & $3 . .80$ & 0.17 & 0.60 & $1 .+40$ & 0.20 & $\pi$ \\
\hline $1 . .40$ & 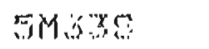 & 0.80 & 4.00 & 0.20 & 0.93 & 1.90 & 0.20 & 60 \\
\hline L.4l. & $543+5$ & 0.90 & 8,60 & $0.1,6$ & 0.73 & 2.00 & 0.80 & \\
\hline
\end{tabular}

Chit ina Valley Area

Rock Samples

\begin{tabular}{|c|c|c|c|c|c|c|}
\hline \multicolumn{7}{|l|}{ Map } \\
\hline $\begin{array}{l}\text { No. } \\
E\end{array}$ & $\begin{array}{l}\text { Field No. } \\
\text { OE } 7 \text { A AR }\end{array}$ & $\begin{array}{l}u p p m \\
0 .\end{array}$ & $\begin{array}{l}\text { Th ppm } \\
i .48\end{array}$ & $\begin{array}{l}\text { U/Th } \\
0 .\end{array}$ & $\begin{array}{l}\% K_{2} \mathrm{O} \\
1.45\end{array}$ & $\begin{array}{l}\text { cps } \\
65\end{array}$ \\
\hline$E 2 k$ & $5 K 65 \mathrm{~K}$ & 0 & 1.50 & 0 & 1.80 & 60 \\
\hline E:3R & $5 K \& 45$ & 0. & 3.00 & 0. & 1.38 & 50 \\
\hline EAF & ラバるダ & 0.40 & 3.00 & 0.13 & 1.51 & 60 \\
\hline 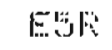 & 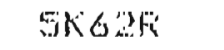 & 0. & 0.20 & 0. & 1.73 & 60 \\
\hline$E G R$ & BE & 0 & 4.30 & 0 & 0.77 & $7 \%$ \\
\hline E.7R & जEsqR & 1.00 & 5.00 & 0.32 & 0.283 & \\
\hline$E B F_{i}$ & 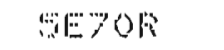 & & 0.4 & & 1.52 & 90 \\
\hline$E 9 F$ & تEフ & 1.60 & $x .00$ & 0.53 & 0.80 & 50 \\
\hline EI.OFi & FE & 1.000 & 1.30 & 0.77 & 0.76 & 80 \\
\hline 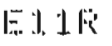 & SKG\&ठR & 0. & 2.50 & 0 & 2.31 & 30 \\
\hline E1.21 & 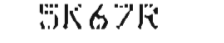 & 0 & 2.30 & 0. & 2.40 & 85 \\
\hline E13/ & GKGBR & 0 & $L .80$ & 0. & 1.81 & \\
\hline$E \perp 4 F$ & $5 K 54$ F & 0. & $1.1,+30$ & 0. & 2.63 & 78 \\
\hline$E d F$ & 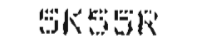 & 1.00 & 7.30 & 0.1 .4 & 2.54 & 学管 \\
\hline E16li & sE 60 Fi & $\underline{2} .010$ & 0.90 & 2.78 & $0.8 \%$ & 10 \\
\hline EJ.7li & GEしER & $2+52$ & $\% .30$ & 0.34 & 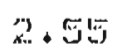 & 90 \\
\hline E.18li & $S E 63 \mathrm{Fi}$ & 0.10 & 1.80 & 0.22 & 1.32 & 75 \\
\hline F.1.9. & $5 E 6 \&$ & 2.00 & 3.80 & 0.5 .3 & 0.97 & 70 \\
\hline ENOF & 战56R & 0.40 & 4.80 & 0.08 & 2.00 & \\
\hline E2liti & "̈KらフRA & 1.90 & [3. & 0.22 & 1.1 .6 & \\
\hline E22 & 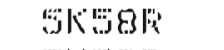 & 1.30 & 6.80 & 0.26 & 2.53 & \\
\hline E. 230 & 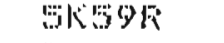 & 1.00 & 7.30 & 0.1 .4 & 2.20 & 100 \\
\hline$\because 2 A F^{\prime}$ & 四しフF & 0 & 3.30 & () & 2.10 & 90 \\
\hline L L I IF & $5 F 62 \sqrt{4} A$ & 0. & 1.30 & 0 & 1.82 & \\
\hline LEK & 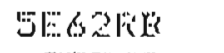 & 0.90 & 4.30 & 0.91 & 1.85 & \\
\hline 1.3F & $5 E 73 \%$ & 0 & 0.40 & ८. & 2.40 & 36 \\
\hline L. 43 & $5 F>3 F E$ & 0. & 0.60 & 0 & 0.50 & $1: 3$ \\
\hline I...5F & 5M15.3F & 0. & 1.50 & 0. & 1.34 & 190 \\
\hline $1.6 \sqrt{i}$ & 5M155F & 0. & 1.000 & 0. & 2.00 & 80 \\
\hline 1...7下 & $5 K 50 R$ & 0 & 3.00 & 0. & 300 & 90 \\
\hline I...BR & $5 K^{\prime} 51 \%$ & 1.40 & $2+830$ & 0.50 & 2.48 & 55 \\
\hline $1 . .9 F^{\prime}$ & $5 E 6 O R$ & 0.70 & 0.40 & 1.75 & 0.20 & 55 \\
\hline 1.... J. (OF & SKIIR & 0.0 & 0 & & $0.0 \%$ & 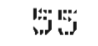 \\
\hline $1 \ldots 11 \mathrm{R}$ & 枵上 $8 F$ & 0.7 & 0. & & 0.39 & 59 \\
\hline L. I. 2 R & :EE & 0 & 0.80 & 0 & 0 & 55 \\
\hline 1...I. $3 F^{2}$ & WEAB & 0.40 & 1.30 & 0.31 & 0.50 & 55 \\
\hline
\end{tabular}


Eagle-Charley River Area

Sediment and Water Samples

\begin{tabular}{|c|c|c|c|c|c|c|c|c|}
\hline $\begin{array}{l}\text { Map } \\
\text { No. } \\
\text { MII. }\end{array}$ & $\begin{array}{l}\text { Field No. } \\
\text { idsos }\end{array}$ & $\begin{array}{l}\text { U pPM } \\
\text { (RAA) } \\
0.90\end{array}$ & $\begin{array}{l}\text { Th ppm } \\
1.60\end{array}$ & $\begin{array}{l}\text { U/Th } \\
0.56\end{array}$ & $\begin{array}{l}8 \mathrm{~K}_{2} \mathrm{O} \\
8.00\end{array}$ & 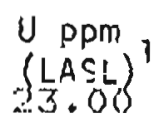 & $\begin{array}{l}\text { U } P P b \\
(\text { LASL) } \\
(.2 .)\end{array}$ & cps \\
\hline$M 2$ & 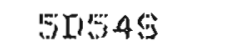 & 1.30 & $12 \geq 0$ & 0.76 & ] 4.50 & 4.00 & $0.6 \mathrm{~B}$ & \\
\hline$M 3$ & 91564 & 2.00 & 1.30 & 1.51 & 5.00 & & & \\
\hline$M A$ & 5026 & 1.60 & J. .60 & 1.00 & 28.50 & & 0.00 & \\
\hline$M 5$ & 5513.5 & 0.60 & $\underline{1}=70$ & 0.35 & 20.50 & 8.60 & 0.55 & 90 \\
\hline$M 6$ & 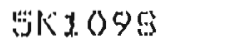 & 1.00 & 1.50 & 0.67 & 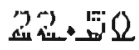 & & 0.45 & 200 \\
\hline$M \%$ & ธパ」08S & 0.90 & 1.60 & 0.56 & 5.00 & 75.00 & 0.10 & 800 \\
\hline$M O$ & $5 k 1075$ & 0.90 & 1.60 & 0.56 & 5.50 & 15.60 & 0.20 & 100 \\
\hline$M$ & 58025 & 2.00 & $1 .=0$ & 1.67 & 4.50 & & 0.20 & 80 \\
\hline$M \pm 0$ & 51065 & 0.90 & 3.60 & $0.5 \mathrm{~s}$ & 15.50 & & 1.40 & $10 \%$ \\
\hline$M 1 x$ & 胀1055 & 1.50 & 1.50 & L. . 00 & 14.50 & & 0.90 & 120 \\
\hline 似等 & ら 1035 & 1.20 & 1.50 & 0.80 & 4.00 & & $3+20$ & \\
\hline$M 13$ & 6035 & 1.40 & $L \perp 7 \Omega_{2}$ & 0.82 & 1.0 .00 & & & \\
\hline$M 1 A$ & تKRIOSG & $0.83<$ & ง. .50 & 0.93 & 14.00 & & 0.35 & 80 \\
\hline$M 15$ & 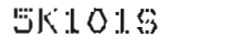 & 1.30 & 1.20 & 0.76 & 4.50 & & 1.00 & 80 \\
\hline M. 6 & $5 k 1005$ & 3.10 & 1.49 & 1.41 & 11.00 & & 1.80 & 80 \\
\hline$M 1 \%$ & oras & 0.70 & 1.30 & 0.54 & 1.00 & & 0.80 & \\
\hline MII 8 & 31045 & 1.830 & 1.30 & 1. .38 & 1.00 & & 1.30 & \\
\hline
\end{tabular}

Healy D-I Quadrangle and Vicinity

Sediment Samples

\begin{tabular}{|c|c|c|c|c|c|}
\hline $\begin{array}{l}\text { Map } \\
\text { No. }\end{array}$ & Field No. & $\begin{array}{l}U_{(R A A)} P P m \\
1\end{array}$ & Th & II/Th & $\% k_{2} n$ \\
\hline 1001 & Wlidobs & $2 . \%$ & 6.90 & 0.39 & 1.62 \\
\hline 1002 & WoloOs & 2.00 & 20.30 & 0.1 .3 & 1.56 \\
\hline 1003 & Wo:1003 & 2.108 & 90.00 & 0.13 & $\therefore$ \\
\hline 100 A & WG1OOA & 1.40 & 1.1 .00 & 0.13 & 2.80 \\
\hline 1000 & WG⿺⿻一𠃋 & 3.40 & 10.00 & 0.34 & 2.17 \\
\hline 1006 & 401006 & 0 & & & 2.35 \\
\hline 1007 & WOL 1007 & 0 & 4.40 & 0 & 1. .74 \\
\hline $100 \theta$ & WE1.00s? & 1.00 & 6.80 & 0.116 & 2.31 \\
\hline 1009 & WG:1009 & 1.20 & 8.30 & 0.14 & 1.34 \\
\hline 1010 & Whaton & 2.00 & 10.60 & 0.19 & 3.15 \\
\hline LO 11 & WG:LOA I. & 1.00 & 6.60 & $0.1,1: 3$ & 2.09 \\
\hline 1012 & WO 1012 & 2.30 & $35+50$ & 0.06 & 3.03 \\
\hline L013 & WG 1013 & $2+10$ & 16.50 & 0.13 & 3.33 \\
\hline 101.4 & W:10.1.4 & 1.00 & 11.90 & 0.08 & $2+55$ \\
\hline 1015 & WGLOJ & 8.10 & 38.50 & 0.21 & 3.40 \\
\hline 1016 & W[1.01.6 & 8.92 & 22.60 & 0.40 & 3.04 \\
\hline $1.01 \%$ & $W C x: L \perp 7$ & 1.40 & 12.30 & 0.1 .1 & 2.00 \\
\hline $10 \pm B$ & WG10 18 & 1.80 & 13.00 & 0.37 & 2.89 \\
\hline 1019 & WG:101\% & 3.40 & 19.00 & 0.18 & $2+97$ \\
\hline 1.020 & W(: 1020 & 2.50 & 21.50 & 0.12 & 4.50 \\
\hline
\end{tabular}

isee p. A-2. 


\begin{tabular}{|c|c|c|c|c|c|}
\hline $\begin{array}{l}\text { Map } \\
\text { No.: }\end{array}$ & Field No. & $\begin{array}{l}\text { U pPm } \\
\text { (RAA) I }\end{array}$ & Th & $\mathrm{U} / \mathrm{Th}$ & $\% \mathrm{~K}_{2} \mathrm{O}$ \\
\hline & W010.1 & & $.6+50$ & $\langle .47$ & $2 y$ \\
\hline $10>2$ & WGLO? & 5.90 & 19.50 & 0.35 & 2.88 \\
\hline $100-3$ & $w(B 10) 3$ & 1.00 & 2.50 & 0.40 & 2.17 \\
\hline 1024 & 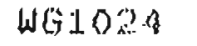 & 1.80 & 5.00 & 0.36 & 4.00 \\
\hline 2025 & WGI.025 & 1.50 & 1.4 .00 & 0.1 .1$. & 4.02 \\
\hline 1026 & WG]. 026 & 8.60 & 35.00 & $(j, 28)$ & 3.06 \\
\hline 1027 & W010. $02 \%$ & 3,50 & 44.00 & 0.19 & $x .083$ \\
\hline 1028 & 401028 & 10.00 & 10.00 & $y .00$ & 3.47 \\
\hline 1029 & W01028 & 3.00 & $1.3+10$ & 0.25 & 2.07 \\
\hline .1030 & WG1030 & $\therefore .10$ & 2.00 & 1.05 & $2 \cdot 62$ \\
\hline $10.3 \%$ & 小 61.031 & $2+00$ & 5.50 & 0.36 & 2.55 \\
\hline 10.32 & W01032 & 1.00 & 7.50 & 0.13 & 2064 \\
\hline 1033 & WG do33 & 0.830 & $\% .50$ & 0.1 .1 & 2.48 \\
\hline 1034 & $w(310.34$ & $1 ., 1: 30$ & 1.2 .50 & 0.12 & 2.00 \\
\hline 1035 & W61 10.35 & $\mathrm{~L} \cdot 40$ & 3.00 & 0.47 & 2.86 \\
\hline 10.35 & W610:36 & 2.1 .0 & 7.00 & 0.30 & 2.05 \\
\hline 1034 & $w(.30 .3 \%$ & 1.60 & 11.00 & 0.16 & 1.68 \\
\hline 1038 & WG1038 & 1.00 & I. 4.50 & 0.07 & 1. $.9 \%$ \\
\hline 1039 & W010.39 & 2.50 & 14.50 & 0.17 & $2 \cdot 36$ \\
\hline 2040 & Wlito:40 & 2.60 & 9.50 & 0.27 & 2.18 \\
\hline 1041 & WO: $104 !$ & 7.00 & 6.80 & 1.08 & 3.96 \\
\hline 1042 & Whido4a & 3.20 & 15.50 & 0.21 & $2+67$ \\
\hline 1043 & WO1043 & 2.70 & 8.50 & 0.32 & 1. . 46 \\
\hline 1044 & 40.1044 & 3.00 & 6.50 & 0.46 & 1.66 \\
\hline 1045 & WE.LOAS & 2.80 & $15+00$ & 0.1 .9 & 1.98 \\
\hline 1046 & $W G 1046$ & 2.80 & 1.50 & 0.22 & 2.07 \\
\hline 10.47 & W & 4.00 & 20.00 & 0.20 & $2 x+1$ \\
\hline $10 \wedge 8$ & WG10AB & $50+90$ & 15.50 & 0.38 & $\therefore .83$ \\
\hline 1049 & Wh1049 & $1+20$ & 1.8 .00 & 0.07 & 3.06 \\
\hline 1.050 & W61,050 & 2.10 & 12.00 & 0.17 & 3.30 \\
\hline $103 !$ & W 30051 & 2.20 & 10.00 & 0.22 & 3.50 \\
\hline $105 ?$ & WGLOS? & 0 & 7.50 & 0 & 3.04 \\
\hline 1053 & WG $1.0 \mathrm{E} ; 3$ & 0.50 & 21.50 & 0.04 & 4.27 \\
\hline J. 054 & W1:1054 & 0 & 9.00 & 0. & 206 A \\
\hline 1035 & Wo10.5 & 1.60 & 1.9 .00 & 0.08 & $\therefore+35$ \\
\hline 10,56 & WG1056 & 0.90 & 16.50 & 0.05 & 2.03 \\
\hline 1057 & W01057 & 1.80 & 10.00 & 0.18 & -3.48 \\
\hline 1058 & w01058 & 1.00 & 1.4 .60 & 0.07 & 3.19 \\
\hline 1059 & Wo J.059 & 1.20 & 1.4 .00 & 0.09 & 2.95 \\
\hline J. 060 & W61060 & 3.10 & 23.30 & 0.1 .3 & 3.18 \\
\hline $106 \%$ & WG106\% & 0.90 & 1.4 .60 & 0.06 & 3.37 \\
\hline 1062 & WG to $02 ?$ & 0. & $12 \cdot 100$ & 0 & $3.1 \%$ \\
\hline 1063 & WOLOB. & ). & 16.00 & 0. & 1.83 \\
\hline 1.064 & WC1.06 & 0.30 & 1.3. 30 & 0.02 & 3.2 .4 \\
\hline $1060 \%$ & $4(41065$ & 0.30 & 21.30 & $0.0 \%$ & $2+72$ \\
\hline 1066 & WG1086 & 1.50 & 1.6 .60 & 0.08 & 3.59 \\
\hline 1067 & Wentos\% & 1.50 & 16.30 & 0.09 & 3.80 \\
\hline 1068 & $\omega 61068$ & 0. & 31.880 & 0. & 4.17 \\
\hline $106 \%$ & WGto6\% & 〈。 & ס0.7. & (). & 3.79 \\
\hline 1070 & WG1.070 & 0.90 & $1 \% .80$ & 0.05 & 3.61 \\
\hline 1071 & WE1.07.1. & 0. & 10.00 & 0 & 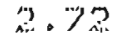 \\
\hline
\end{tabular}

1. See $p \cdot A-2$. 


\begin{tabular}{|c|c|c|c|c|c|}
\hline $\begin{array}{l}\text { Map } \\
110.79 \\
1072\end{array}$ & $\begin{array}{l}\text { Fiald Mo } \\
\text { WGlot? }\end{array}$ & $\begin{array}{l}\text { U ppm } \\
(R M A) \\
0.70\end{array}$ & $\begin{array}{l}\text { Th } \\
16.80\end{array}$ & $\begin{array}{l}11 / T h \\
0.04\end{array}$ & $z_{2} k_{0}$ \\
\hline $10 \% 3$ & W61073 & 1.00 & 39.50 & 0.0 .3 & 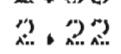 \\
\hline 1074 & $W(31074$ & 1.20 & 34.30 & 0.03 & 2.26 \\
\hline 1075 & WC10\% & 0.80 & 31.80 & 0.03 & 2.38 \\
\hline 1076 & W[j10\%6 & 1.80 & 1.3 .50 & 0.10 & 2.37 \\
\hline 1077 & WGL107" & 1.00 & 7.50 & 0.13 & 3.01 \\
\hline 1078 & WO. 1.078 & 0.70 & 20,30 & 0.0 .3 & 3.66 \\
\hline 1079 & WG10>3 & 0.70 & $22+80$ & 0.03 & 2.71 \\
\hline 1080 & Wot 000 & 1.00 & 18.00 & 0.06 & 3.49 \\
\hline 1081 & WGL 081 & 0 & 1.8 .00 & 0. & 2.32 \\
\hline 1082 & $W(3.082$ & & 14.3 & & 3.83 \\
\hline 1083 & WG108.3 & 0. & 9.30 & 0. & 2.603 \\
\hline 1084 & WG1084 & 0. & 28.30 & 0 & 4.14 \\
\hline 1083 & Wo1 $08 \%$ & 3.40 & 20.80 & $0+16$ & 3.41 \\
\hline 1086 & WGtOB & 1.60 & 19.50 & 0.09 & $\because .6 \%$ \\
\hline 1087 & W[j1 1087 & 1.00 & 1.9 .50 & $0.2 \mathrm{~J}$ & 3.50 \\
\hline 1.088 & Weto 188 & 1.00 & $26 \cdot 20$ & 0.04 & 1.08 \\
\hline 1089 & WOLOSO & $1+10$ & 4.3 .00 & 0.1 .0 & 5.03 \\
\hline 1090 & WOLOSO & 0. & 1.6 .30 & 0 & $2+\$ 3$ \\
\hline J. 091 & WG109.1. & 1.30 & 20.90 & 0.06 & 3.69 \\
\hline 1092 & 46.1092 & $x, 10$ & 19.00 & 0.06 & 3.00 \\
\hline 1093 & WG1003 & 1.30 & 27.80 & 0.05 & 4.54 \\
\hline 1094 & WGioga & 1. . .1. 0 & 30.80 & 0.04 & $4+85$ \\
\hline 1.098 & WG1.08\% & 2.50 & 24.80 & 0.10 & $4.4 A$ \\
\hline J 096 & WG1096 & 2.80 & $28 \cdot 30$ & 0.10 & 1.1 .9 \\
\hline 1.097 & W0.31097 & 2.90 & 34.30 & 0.08 & 3.24 \\
\hline 1098 & Wot.098 & 1.30 & 23.30 & 0.06 & 2.27 \\
\hline 1.098 & W6109\% & 2.70 & $23 \cdot 50$ & 0.11 & 3.28 \\
\hline 1100 & 601100 & 1.80 & 19.60 & 0.09 & $4.1 B$ \\
\hline 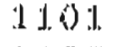 & WEI IOL & 1.30 & 16.50 & 0.08 & 3.93 \\
\hline $21.0 ?$ & WELIO? & 1.50 & $\therefore 4.80$ & 0.06 & $\$ .10$ \\
\hline 110.3 & WG1 to:S & 3.60 & 25.30 & 0.14 & 3.17 \\
\hline 2104 & 601104 & 1.40 & 21.80 & 0.06 & 3.86 \\
\hline 1. 1.05 & W6.1. 105 & 1.40 & 20.50 & $0.0 \%$ & 3.79 \\
\hline . I.OA & $W G \times 1 . \Delta G$ & 1.50 & 16.50 & $0.0 \%$ & 3.22 \\
\hline 1. $1 . \diamond \%$ & WG110\% & 3.90 & 35.00 & 0.1 .1 & 3.28 \\
\hline 1. 1.08 & WG.1.108 & 2.00 & 56.50 & 0.04 & 3.20 \\
\hline 1.109 & LG] & $? \cdot 30$ & 4.1 .00 & 0.06 & .3 .20 \\
\hline L.t. J. 0 & WE1 1.1 .0 & 1. . 50 & 26.00 & 0.06 & 3.16 \\
\hline 1.111 & WG $1 . \perp\rfloor$ & 1.00 & 19.50 & 0.05 & 3.04 \\
\hline 1.1 .2 & WG.1] 12 & 1.40 & 21.30 & 0.07 & 3.30 \\
\hline $1 \pm 1.3$ & $W(91+1,3$ & ป. . . & 13.50 & 0.1 .1 & $2 \cdot 44$ \\
\hline 1114 & Whis 14 & 1.00 & $\mathrm{~L} 2,150$ & 0.08 & 2.90 \\
\hline 11. J & WG.1. 1.5 & 0.70 & 29.30 & 0.02 & 3.42 \\
\hline 1.1. I & Wo. 1.16 & 0.80 & 1. 4.00 & 0.06 & 3.42 \\
\hline 1. 117 & WG 1117 & 0. & 1.8 .80 & 0 & 3.73 \\
\hline 11.18 & WGL. 18 & 1.20 & 14.50 & 0.083 & 2.65 \\
\hline 1119 & $W G 1119$ & $22=20$ & 21.80 & 0.10 & 4.16 \\
\hline 1120 & $W G 1120$ & 3.70 & 31.30 & 0.12 & $3+42$ \\
\hline 121 & WGป 121 & 0.40 & 23.30 & 0.02 & 3.79 \\
\hline 1452 & WG1.22 & 1.30 & 24.30 & 0.05 & 3.6 .1 \\
\hline
\end{tabular}

1. See P. A-2. 


\begin{tabular}{|c|c|c|c|c|c|}
\hline 1. 1.24 & $W G \perp: 24$ & 1.70 & $\begin{array}{l}28+80 \\
30+30\end{array}$ & $\begin{array}{l}0.05 \\
0.06\end{array}$ & $\frac{4.7 \%}{3.04}$ \\
\hline 1225 & WG1125; & 1.30 & 25.80 & 0.05 & 3.82 \\
\hline 1126 & WG: 1.26 & 1.90 & 23.80 & 0.08 & $B+1.5$ \\
\hline 11.27 & WG.L.127 & 0.90 & 18.00 & 0.05 & 2.71 \\
\hline 1128 & W $G, 1 \perp \Omega$ & 1.20 & 37.00 & 0.03 & $x .73$ \\
\hline 1129 & WOL129 & 0.50 & 19.50 & 0.0 .3 & 1.31 \\
\hline 1130 & $W 61130$ & 1.30 & .19 .50 & 0.07 & $3.9 \%$ \\
\hline 1.1 .31$. & WG1 131 & 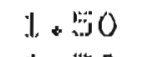 & $27+30$ & 0.05 & 3.03 \\
\hline 1. 1.32 & W.1.1.3? & $\mathrm{J} \cdot 30$ & 18.00 & 0.07 & $4.0 \%$ \\
\hline 1133 & $W[1.133$ & $1 . \% 0$ & $28 \cdot 60$ & 0.00 & 3.42 \\
\hline $1: 1.34$ & WOLI I 34 & $\mathrm{i} .90$ & 6.23 & 0.28 & 2.61 \\
\hline 1235 & Woil 135 & 1. 60 & $43 \cdot 80$ & 0.12 & 2.99 \\
\hline 1. 1. & WE.1.36 & 2.30 & 13.00 & 0.1 .9 & 1.97 \\
\hline 1. 1.37 & WG I. 1.37 & 2.40 & 3.3 .300 & $0.0 \%$ & $3 \cdot 11$ \\
\hline 1138 & WG 1138 & 1.90 & 24.00 & 0.08 & 3.17 \\
\hline 1139 & 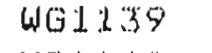 & 1.90 & $2 \%, 50$ & 0.08 & $2 \cdot 76$ \\
\hline$(140$ & W61.110 & 2.20 & $35+30$ & 0.06 & $2 \cdot 13$ \\
\hline$\lfloor 1.4\rfloor$ & WGI 1 A I & $23+40$ & $3,3,50$ & 0.07 & $\because 36$ \\
\hline 1. 1. 4.2 & WE1 LA? & 1.70 & 24.00 & $0.0 \%$ & $\Rightarrow$ \\
\hline 1.4 .3 & WG114 & 1.80 & $25+30$ & 0.08 & 3.62 \\
\hline $1 \perp A 4$ & WGI I \& 4 & 1.40 & 320.30 & 0.06 & 3.33 \\
\hline 1145 & W. 1145 & 0.50 & $13 \cdot 30$ & 0.01 & 2.69 \\
\hline 1146 & Wej:1 146 & 1.10 & 16.80 & $0.0 \%$ & 2.44 \\
\hline $114 \%$ & WGIX $4 \%$ & 2.40 & $1 \% .50$ & 0.1 .8 & 3.46 \\
\hline .1. 1.48 & מ⿴囗十⺝ 198 & $\therefore \cdot()$ & 0 & & $\Rightarrow .88$ \\
\hline I. 1.49 & WOI 1 49 & $1.30)$ & 18.80 & 0.07 & $\therefore .83$ \\
\hline 11.50 & W(1) & 1.30 & 20.00 & 0.06 & $2 \cdot 37$ \\
\hline I. 1.101 & woll Is] & 0.70 & 14.60 & $0.01: 3$ & 3.53 \\
\hline I. $13 \%$ & WGD 13 & 1.60 & $26+30$ & $0.0 t$ & 4.00 \\
\hline 21.53 & 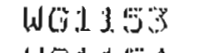 & 1.90 & 15,00 & 0.12 & 3.00 \\
\hline 11.64 & WG1 1.64 & 3.80 & 31.00 & 0.12 & $2+31$ \\
\hline 1.1. 1. & WEII 5 & 0.70 & 13.80 & 0.05 & $\therefore .30$ \\
\hline 1.1. 60 & W011 & 0.70 & 11.00 & 0.06 & 2.34 \\
\hline $310 \%$ & $W(3215 \%$ & 0.40 & 14.50 & 0.03 & 2.30 \\
\hline 1.1. 158 & W61.158 & 2.40 & 1.4 .50 & 0.17 & 2.832 \\
\hline $115 \%$ & WG 159 & 1.40 & 18.30 & 0.08 & 2.67 \\
\hline 1. 160 & whed too & $\therefore .20$ & 1.3 .80 & 0.16 & 2.63 \\
\hline a $1 \& 1$ & $W G \perp 161$ & 2.00 & 1.7 .00 & 0.12 & 2.98 \\
\hline l. J. 6 & WGLC\&? & 3.30 & 18.50 & 0.18 & 3.00 \\
\hline 116.3 & WG 1. 1. & 3.50 & 40.30 & 0.09 & 3.20 \\
\hline 1164 & $w 6116.4$ & 4.30 & $22+50$ & 0.06 & \pm .20 \\
\hline L. L 65 & W6. I L 60 & 2.20 & $29 \cdot 30$ & 0.08 & 4,50 \\
\hline 11.160 & Whit it & $3+20$ & 29.30 & 0.08 & 3.45 \\
\hline 1167 & w 61.167 & $2+20$ & 1.83 .80 & 0.12 & 3.98 \\
\hline 1168 & Wo $1.16 \theta$ & 4.50 & $16 \cdot 80$ & 0.27 & J. 55 \\
\hline 11.89 & W01169 & 2.10 & 21,50 & 0.10 & 3.17 \\
\hline 1.170 & WExt:0 & 2.20 & 99.00 & 0.04 & 2.84 \\
\hline 1.71 & WG11.1. & 2.00 & 38.00 & 0.05 & 2.70 \\
\hline 1.1.72 & WELI\%? & $1 .+6<\rangle$ & 28.50 & 0.06 & 2.74 \\
\hline 1.173 & WG117z & 1.90 & 26.50 & 0.07 & 3.13 \\
\hline 1. 1.74 & WO117A & 1.80 & 33.70 & 0.0 .3 & 3.45 \\
\hline
\end{tabular}

1. See $p . A-2$. 


\begin{tabular}{|c|c|c|c|c|c|}
\hline $\begin{array}{l}\text { Map } \\
\text { No. }\end{array}$ & Field No. & $\begin{array}{l}\text { U PPM } \\
\text { (RAA) }\end{array}$ & Th & $U / T h$ & $\% k, 0$ \\
\hline 1175 & WGI 1.75 & 3.30 & 7.00 & 0.47 & 2.44 \\
\hline 1176 & W61 176 & 1.70 & 26.75 & 0.06 & 3.97 \\
\hline 1. 1777 & WG」177 & 1.90 & 10.00 & 0.19 & 1.90 \\
\hline d. $1>8$ & $W G \perp 170$ & 1.10 & 10.00 & 0.1 .1 & 3.08 \\
\hline 1.79 & WOLI 179 & 1.90 & 11.00 & $0.1 \%$ & 3.54 \\
\hline 1.1 .80 & WG」. 180 & 1.00 & 9.75 & 0.10 & 2.04 \\
\hline 1181 & Wtid 181 & 1.30 & 29.75 & 0.04 & 3,12 \\
\hline 1182 & WE1.82 & 0.60 & 7.0\langle\rangle & 0.09 & 1.59 \\
\hline 11.83 & Wo 1 y 8.3 & J. . 40) & 18.25 & 0.08 & 2.70 \\
\hline 1184 & WE1.184 & 3.150 & 16.25 & 0.09 & 3.80 \\
\hline 1285 & WG1185 & 1.70 & 1.4 .00 & $0+12$ & 2.47 \\
\hline J.1 186 & WGI 186 & 1.00 & 21.00 & 0.09 & 2.99 \\
\hline 1287 & WG1.67 & 2.10 & 17.50 & 0.12 & 3.56 \\
\hline 1183 & W & 1.80 & 3.75 & 0.43 & $1.4 O$ \\
\hline 1189 & $W G \perp 1, B Y$ & 2.60 & 11.25 & 0.23 & 3.41 \\
\hline 11.80 & WOI 150 & 2.60 & 10.50 & 0.25 & 2.84 \\
\hline $.125: 1$ & WGI:191 & 2.30 & 16.00 & 0.1 .4 & $2 . \%$ \\
\hline 11.92 & W[: 1 19? & 2.20 & $5+75$ & 0.38 & 2.75 \\
\hline .1. 193 & $w G 1193$ & 2.20 & 6.00 & 0.37 & 2.08 \\
\hline 1194 & WGI 194 & $2 \cdot 10$ & 8.74 & 0.24 & 2.46 \\
\hline $1 \mathrm{~L} \%$ & WELL 195 & $2 \cdot 1.0$ & 25.00 & $0 .(>B$ & 4.22 \\
\hline 1.196 & WCi.1. & 3.20 & 12.5 & 0.26 & 3.82 \\
\hline 1.1 .97 & W[1.197 & $\therefore .80$ & 7.50 & $0.3 \%$ & 1.95 \\
\hline 1.98 & WG.1198 & 1.20 & 7.75 & 0.15 & 3.53 \\
\hline 11.95 & WG 11.99 & 1. . 30 & 24.00 & 0.05 & 3.07 \\
\hline 1200 & WG 1200 & 1.50 & 6.75 & 0.22 & 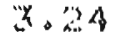 \\
\hline 1201 & WG1201 & $1.1,0$ & 32.25 & 0.0 .3 & 1.65 \\
\hline 1202 & 601202 & & 1.5 .75 & & 340 \\
\hline 1203 & WG:203 & 0.50 & 1.1 .75 & 0.04 & 3.063 \\
\hline 1.204 & W1.20.4 & 1.40 & 26.50 & 0.05 & 1,90 \\
\hline 1.305 & W61.205 & $\therefore \cdot 10$ & 18.20 & 0.1 .2 & 3.12 \\
\hline 1.206 & W. $12\langle 2\rangle$ & 2.20 & 15.20 & 0.1 .4 & $2.7 \%$ \\
\hline $1.20 \%$ & WG:1207 & 1.70 & 25.70 & 0.07 & 4.38 \\
\hline 1208 & Wo120s & 0.80 & 1.4 .20 & 0.06 & 2.78 \\
\hline
\end{tabular}

1. See p. A-2. 


\section{HISTOGRAMS}

Shown below are histograms of uranium values from sediment samples analyzed by Resource Associates of Alaska ( $(B$ RAA) and Los Alamos Scientific Laboratories (U LASL) and water samples $\left(\mathrm{U}^{\mathrm{H}} \mathrm{O}\right)$ analyzed by LASL. The total number of samples is limited to those for which there are no data for these categories. The printed interval designations are the lower limits of class intervals.

\section{Granite Mountain area}

U RAA

(), 6.000 ?

$90.000)$

$80.0003 \times$

$0,0.000,1$

75.000 ,

$70.000 ;$

$65.000 ;$

$60.000 ;$

$55.000)$

$50.000 \%$

$4.000)$

$40.000)$

$35.000)$

30.000 ;

$29.000) *$

$20.000) * *$

$15.000)$ 水水次

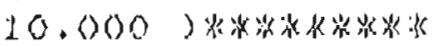

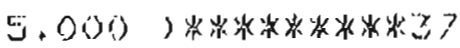

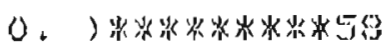

MEATR B. 84:

$S \quad[M V 9.178$

N 113 . $\checkmark$ LASL

$95.0(0))$

$90.000) *$

$85.000) *$

80.000 )

$75.000>$

70.000

$65+000)$

$60.000,3$

$55.000 ?$

$50.000>*$

$45.000 ? *$

$40.000) * *$

$35.000) * *$

$30.000>*$

$250,000) \times 3$

$30.000) * 2 * 3 * 2 * 2$

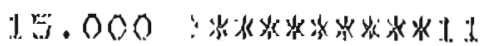

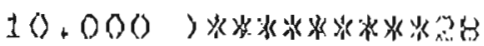

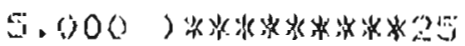

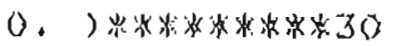

MEAN 13.581

5 [IEU T.9.984

N 1.12 .
U

$$
\mathrm{H}_{2} \mathrm{O}
$$

$2.400)$

$2.250)$

$2.100>$

$1.950>*$

$1.800>*$

$1.650,12 \times$

$1.500) * * *$

1.350 )****

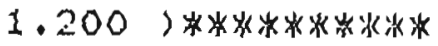

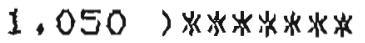

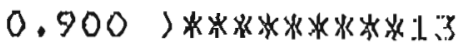

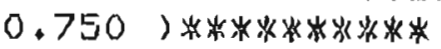

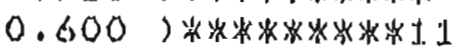

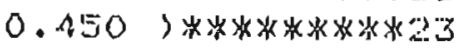

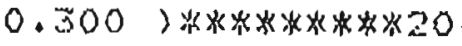

$0.150) * * * * * * *$

$-0.000) * * *$

$-0.150)$

$-0.3001$

-0.4 (iin)

MEAN 0.745

5 IIEU 0.41 .9

N 112 . 
Darby Mountains

U RAA

\begin{tabular}{|c|c|}
\hline 85.000 & ) \\
\hline 80.000 & ) $*$ \\
\hline 78.000 & ) \\
\hline 70.000 & s \\
\hline 65.000 & ) \\
\hline 60.000 & )末为 \\
\hline 55.000 & 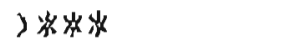 \\
\hline 50.000 & $* * *$ \\
\hline 45.000 & ) 水 \\
\hline 40.000 & ) \\
\hline 35.000 & ) 好珰为 \\
\hline 30.000 & ) $x_{*}$ \\
\hline 25.060 & $3 * x^{2}$ \\
\hline 20.000 & 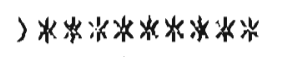 \\
\hline 15.000 & 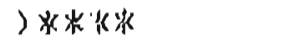 \\
\hline 10.000 & 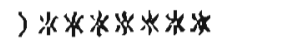 \\
\hline 5,000$)$ & 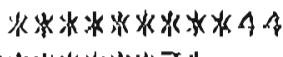 \\
\hline ）。）永冰出 & 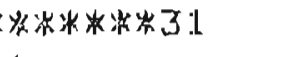 \\
\hline $\begin{array}{l}9.000 \\
10.000\end{array}$ & ) \\
\hline
\end{tabular}

MEAN 1 A. 108

$\because 8100.60 .345$

N 14.3.
11 I.ASL

\begin{tabular}{|c|c|}
\hline 1.27 .900 & 1 \\
\hline 120.000 & l \\
\hline 11.3 .500 & $>$ \\
\hline 100.000 & $0) * *$ \\
\hline 97.500 & \\
\hline 90.000 & $>$ \\
\hline $92 \cdot 300$ & ) \\
\hline 70.000 & ) \\
\hline$\therefore 7.600$ & ) $*$ \\
\hline 60,000 & >氺 \\
\hline 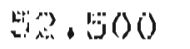 & ) \\
\hline 45.000 & 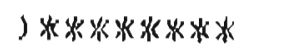 \\
\hline 37.500 & ) $* * * * * *$ \\
\hline 30.000 & 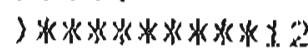 \\
\hline 23.500 & ) \\
\hline 1.30000 & 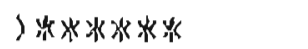 \\
\hline 7.600$) x$ & 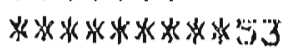 \\
\hline ， J 水水 & 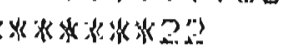 \\
\hline-7.000 & \\
\hline$\cdots+1: 000$ & ) \\
\hline MEAN 20 & 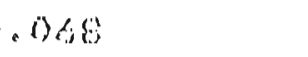 \\
\hline$\Rightarrow$ nEu ts & 5.875 \\
\hline N III 3 & \\
\hline
\end{tabular}

Selawik Hills Area

UAA

32.000,

$30.000)$

$20.000)$

$26.000>*$

$24.000)$

22.000 )

20.000 )

$19.000)$

16.000 )

$14.000) * *$

12.000 )

10.000, 必水皮然

Q.000) 水水

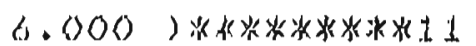

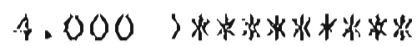

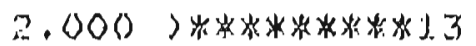

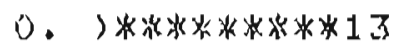

MEAIN E. EA J.

C; KIEV A. 650

is 56 .
U LASL

1.05, 000$)$

$97.500,12$

90.000 ?

$32,500 ?$

$75.00(0)$

$67.500)$

$60.000)$

$52.500) * 2$

45.000 )

$37.500) *$

30.000 ) 秋为

$22.500>* *$

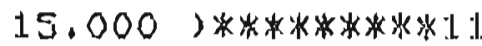

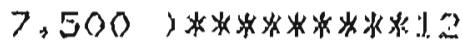

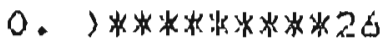

$-7.500)$

-15.000 ?

MFAN 13.630

5 [IEU 1.5 .860

$N 56$,
U<smiles>[18OH]</smiles>

$2: 550)$

2.400 )

$2.250, *$

$2.100 J *$

$1.950 ;$ 水次

$1.8001,3$

1.650)冰水水水

$1.500): 4$

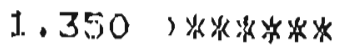

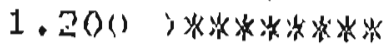

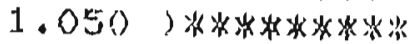

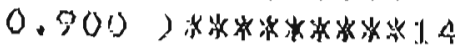

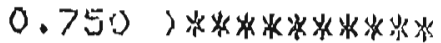

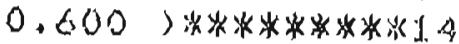

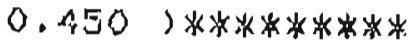

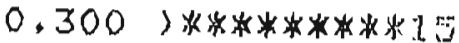

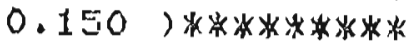

0). * x^{*} *$

-0.150 ,

-0.300 )

MEAN O.834

5 IIEU 0.528

N 113 .

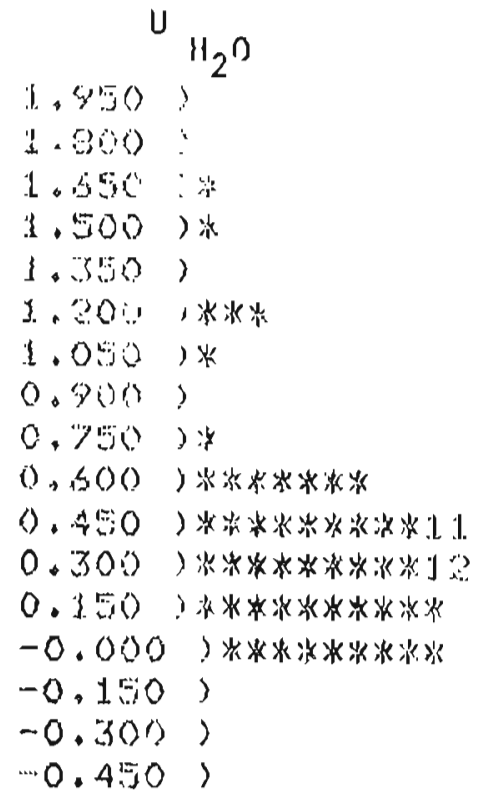

TIEAN O. \$5O

5 IIEU 0.382

iv 58 . 
U RAA

\begin{tabular}{|c|c|}
\hline 60.000 & ) \\
\hline 56.000 & $; * * * *$ \\
\hline 52.000 & $>$ \\
\hline 48.000 & ) \\
\hline 44.000 & ) \\
\hline 40.000 & ) \\
\hline 38.000 & $2 *$ \\
\hline 32.000 & ) 水 \\
\hline 28.000 & ) 次米米 \\
\hline 2.1 .000 & )水 \\
\hline 20.000 & ) 必必必 \\
\hline 16.000 & ) 水必水* \\
\hline 12.000 & 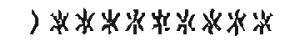 \\
\hline 8.000 & 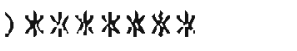 \\
\hline 4.000 & 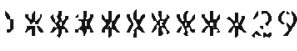 \\
\hline 0.1 岁次 & 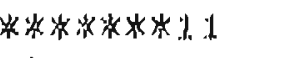 \\
\hline$\cdots .4 .000$ & ) \\
\hline 9.000 & ) \\
\hline
\end{tabular}

MENN J 3.443

S IIEU 12.771

1! 75.
U

LASL

\begin{tabular}{|c|c|}
\hline & \\
\hline $\begin{array}{l}85.000 \\
80.000\end{array}$ & ; \\
\hline 75.000 & ) $* *$ \\
\hline 70.000 & ) \\
\hline 65.000 & ) $*$ \\
\hline 60.000 & ) $* *$ \\
\hline 55.000 & ) $k$ \\
\hline 50.000 & )为求 \\
\hline 45.000 & ) \\
\hline 40.000 & )次束水策 \\
\hline 35.000 & )水米** \\
\hline 30.000 & )决必米 \\
\hline 25.000 & ) 必水冰**水 \\
\hline 20.000 & ) 称州办 \\
\hline 15.000 & 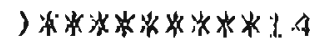 \\
\hline 10.000 & 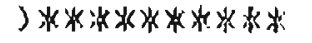 \\
\hline 5.000 & 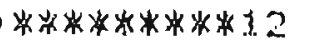 \\
\hline ) & $k * * * * * * *$ \\
\hline
\end{tabular}

MEAN 23.284

$S$ [IE:U 1.9 .067

N 75.
$\mathrm{U} \mathrm{H}_{2} \mathrm{O}$

4.500

4.200 )

$3.900) 2 \%$

3.6001

3.300,

$3.000)$ 冰

$2.700) * 2$

$2.400>* * * *$

$2.100) * *$

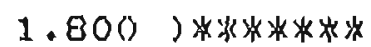

1. $500>* * * * * 2 * x$

$1.200>* * * * * * * * * k$

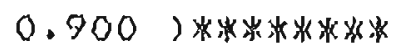

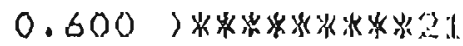

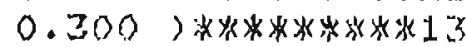

0, ) 水水水水

$-0.3001$

$-0.600)$

MEAN 1, I. J.5

5 IFEU 0.76 ]

N 75.
Copper River Basin

U RAA

2. $: 500 \%$

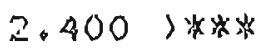

$2.250)$

$2.100) * 3$

$1.950) *$ 皮决

1. .800$) * * *$

$1.860) * 3$

1.500 ) 次水

$1.350) * * * * 4$

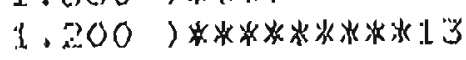

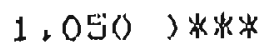

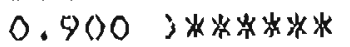

$0.750 \quad ; * 3 * * * *$

$0.600) * 3$

$0.450)$ 皮水

$0.300) * * *$

0.150 )

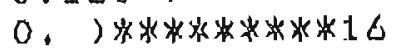

MEAN 0.961

5 IIEU 0.710

$N 7()$.
U LASL

$3.400)$

$3.200, * 6$

$3.000) *$

$2.800)$

$2.600) \times$

$2.400) * * * *$

$2.200) * 2 * 3$

2.000 )

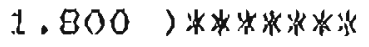

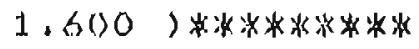

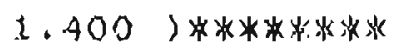

1. .200$) * * * * * * * * 12$

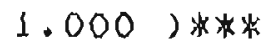

$0.800) * * * * * * *$

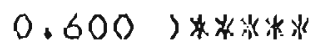

0.4002 2***

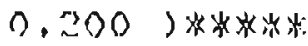

$0 。$, 水

BRE: AN L, 356

6 IIEV $0.67 \%$

N 70 .
$\mathrm{U} \mathrm{H}_{2} \mathrm{O}$

$2.250)$

$2.100)$

$1.950>$ 样

$1.800) *$

1. 650$) *$

1.500)冰水水

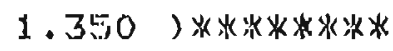

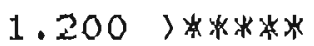

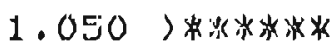

$0.900 ;, * w^{2}$

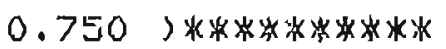

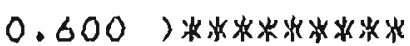

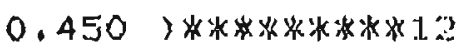

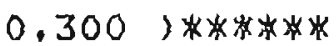

$0.150) *$

0.1

$-0.150)$

-0.300 )

MEAN 0.91 .4

$S$ IIEU 0.420

$N 70$. 
U RAA

\begin{tabular}{|c|c|}
\hline 4.200 & ) \\
\hline 3.900 & ) \\
\hline 3.600 & 1 \\
\hline 3.300 & ) $3(x$ \\
\hline 3.000 & )㖨 \\
\hline 2.700 & $>k_{3}$ \\
\hline 2.400 & ) \\
\hline 2.100 & ) $*$ \\
\hline 1.800 & 了冰* \\
\hline$L .500$ & 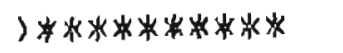 \\
\hline 1.200 & 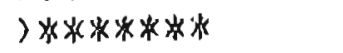 \\
\hline 0.900 & ) $* * * * * * * *$ \\
\hline 0.600 & 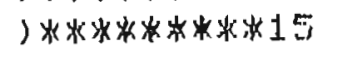 \\
\hline 0.300 & 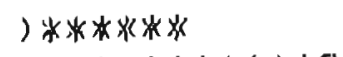 \\
\hline-0.000 & 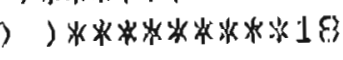 \\
\hline-0.300 & 3 \\
\hline-0.600 & ) \\
\hline-0.900 & 01 \\
\hline
\end{tabular}

MEAN 0.945

5 IIEV 0.965

N 71 .
U LASI.

$(9.900)$

6.400 )

$6.000 ; *$

5.6001

$5.200)$

4.800 )

4.400 )

$4.000>* *$

$3.600) *$

$3.200)$ 水****

$2.800>* * *$

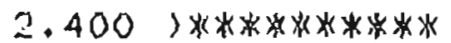

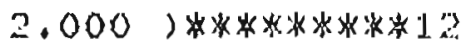

$1.500 \supset * * * * * * * * * * 12$

$1.200)$ )

$0.800) \div * * * * * * * * *$

$0.400>$ 冰*

0.7

ME: AN 2.008

$S$ MEU 0.951

N 71 .
U $\mathrm{H}_{2} \mathrm{O}$

4.200 )

3.9003

3.600 )

$3.300>x$

$3.000>$

$2.7003 *$

$2.400>$

$2.100)$ w

$1.800)$

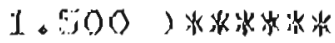

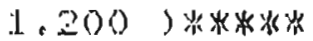

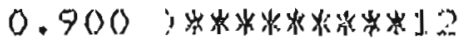

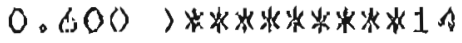

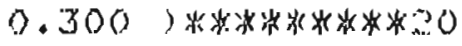

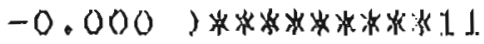

$-0.3001$

-0.800 )

$-0.900)$

MEAN 0.815

5 RIEU 0.607

N $7 \%$. 


\section{CORRELATION MATRIX}

Variable 1 represents uranium, variable 2 represents thorium, and variable 3 represents potassium oxide.

$$
\begin{aligned}
& \text { Granite Mountain Area } \\
& \text { (Coefficient } \geq .215 \text { represents significant correlation.) }
\end{aligned}
$$
1. $\frac{1}{1.00000}$
$\frac{2}{0.31092}$
$\frac{3}{0.30559}$
2. 0.31092
1.00000
0.63778
3. 0.30559
0.63778
1.00000

Darby Mountains

(Coefficient $\geq .208$ represents significant correlation.)
1. $\frac{1}{1.00000}$
$\frac{2}{0.54404}$
$\frac{3}{0.002} \frac{15}{0.31250}$
2. 0.54404
1.00000
0.31250
3. 0.00215
0.31250
1.00000

Selawik Hills Area

(Coefficient $\geq .283$ represents significant correlation.)
1. $\overline{1.00000}$
$\frac{2}{0.44735}$
$\frac{3}{0.33592}$
2. 0.44735
1.00000
0.54408
3. 0.33592
0.54408
1.00000

Zane Hills-Purcell Mountains Area

(Coefficient 267 represents significant correlation.)
1. $\frac{1}{1.00000}$
$\frac{2}{0.47455}$
3
2. 0.47455
1.00000
0.58899
3. 0.58899
0.56517
0.56517
1.00000

Copper River Basin

(Coefficient $\geq 267$ represents significant correlation.)
1. $\frac{1}{1.00000}$
$\frac{2}{0.21841}$
$\frac{3}{0.07743}$
2. 0.21841
1.00000
0.29321
3. 0.07743
0.29321
1.00000

Chitina Valley Area

(Coefficient $\geq .254$ represents significant correlation.)
1. $\frac{1}{1.00000}$
$\frac{2}{0.17175}$
$\frac{3}{0.16597}$
2. 0.17175
1.00000
0.27791
3. 0.16597
0.27791
1.00000 


Eagle-Charley River Area
(Coefficient $\geq .561$ represents significant correlation.)
$\begin{array}{ccc}1 & & 2 \\ \text { 1. } 1.00000 & -0.31310 & \frac{3}{-0.21625} \\ 2 .-0.31310 & 1.00000 & 0.26562 \\ 3 .-0.21625 & 0.26562 & 1.00000\end{array}$

Healy D-1 Ouadrangle and Vicinity

(Coefficient $\geq .181$ represents significant correlation.)
1. $\frac{1}{1.00000}$
$\frac{2}{0.25375}$
$\frac{3}{-0.03084}$
2. 0.25375
1.00000
0.34538
3. -0.03084
0.34538
1.00000 
Appendix D

DETERMINATION OF THORIUM, URANIUM, AND POTASSIUM OXIDE IN STREAM SEDIMENTS AND ROCKS

THORIUM

Digestion

Approximately $0.4 \mathrm{~g}$ of a -80 fraction is digested in a $1: 1$ mixture of hydrofluoric and nitric acids. The residue is then treated with excess boric acid to remove all traces of fluoride, and the remaining residue is taken up into $50 \mathrm{ml}$ of a $\mathrm{IN}$ solution of $\mathrm{HNO}_{3}$; an equal volume of concentrated $\mathrm{HNO}_{3}$ is then added to make an approximate $8 \mathrm{~N}-\mathrm{HNO}_{3}$ solution.

\section{Ion-Exchange Separation}

The $8 \mathrm{~N}-\mathrm{HNO}_{3}$ digestate is then passed through a $50-\mathrm{cm} \times 1.5-\mathrm{cm}$ ion-exchange column filled with Dowex $1 \times 8$ Resin in the chloride form, which has been freshly converted to the $\mathrm{NO}_{2}$ form by running through it $100 \mathrm{ml}$ of $8 \mathrm{~N}_{-} \mathrm{HNO}_{3}$. After all of the digestate has been run through the column, it is washed with another $100 \mathrm{ml}$ $8 \mathrm{~N}-\mathrm{HNO}_{3}$. The column is then eluted with $100 \mathrm{ml} 6 \mathrm{~N}-\mathrm{HCl}$ into a clean beaker. This liberates all the thorium which has been complexed in the resin. The eluate is then evaporated to dryness on a hot plate.

Colorimetry

1) To the dried residue, add $5 \mathrm{ml} \mathrm{IN}-\mathrm{HCl}$ and $5 \mathrm{ml}$ of 2-percent aquous solution of $\mathrm{KMnO}_{4}$, and place on a hot place and evaporate.

2) To this, add $10 \mathrm{ml}$ concentrated $\mathrm{HCl}$ and evaporate.

3) To the white dried residue, add another $10 \mathrm{ml}$ concentrated HCl and 5 drops formic acid and evaporate. This destroys all remaining traces of nitrates which would interfer in the final step.

4) The residue is now treated with $5 \mathrm{ml}$ concentrated $\mathrm{HCl}$ and transferred quantitatively to a $10-\mathrm{m}\}$ vol. flask.

5) Add I $\mathrm{ml}$ of a 0.2 -percent solution of Arsenazo 111 and bring to $10 \mathrm{ml}$ with $\mathrm{H}_{2} \mathrm{O}$.

6) Determine absorbance at 660 nanometers on a Beckman-25 spectrophotometer.

Thorium forms a purple complex with Arsenazo 111. The intensity of the complex obeys Beer's law in the range 0.1 to $1.5 \mathrm{~g} \mathrm{Th} / \mathrm{ml}$.

URAN I UM

Digestion

A l-g sample is digested in I:l $\mathrm{HF} / \mathrm{HNO}_{3}$. Fifteen $\mathrm{ml}$ of each are used three times. The digestate is then filtered with a I-percent $\mathrm{HNO}_{3}$ solution and brought to $100 \mathrm{ml}$.

\section{Extraction}

Five $\mathrm{ml}$ of sample, $5 \mathrm{ml}$ of saturated aluminum nitrate, and $5 \mathrm{ml}$ of ethyl 
acetate are placed into a $25-\mathrm{ml}$ test tube and shaken for 3 minutes. The organic layer is allowed to separate. Then a $0.1-\mathrm{ml}$ aliquat is taken from the organic layer and placed directly on a pellet made from 9 parts $\mathrm{NaF}, 45.5$ parts potassium carbonate, and 45.5 parts sodium carbonate, and allowed to dry on a $\mathrm{Pt}$ dish. it is then placed into the oven for 25 minutes at $650^{\circ} \mathrm{C}$.

Fluorescence

The pellets are then removed from the oven and their concentration is read off the Turner-110.

\section{POTASSIUM OXIDE}

1) Take $0.2 \mathrm{~g}$ sample and $1.0 \mathrm{~g}$ lithium metaborate and fuse for $25 \mathrm{~min}$. at $1000^{\circ} \mathrm{C}$ in graphite crucible.

2) Dissolve pellet in $150 \mathrm{ml}$ of 15 -percent $\mathrm{HNO}_{3}$ using magnetic stirrer; bring solution to $200 \mathrm{ml}$ with distilled water.

3) Determine $K$ on atomic absorption unit as percent $\mathrm{K}_{2} \mathrm{O}$. 


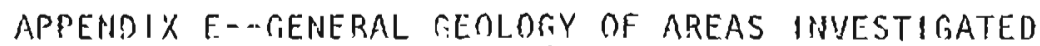

\section{GRANITE MOUINTAIN-HUNTER CREEK PLUTON AREA}

\section{Location}

Granite Mountain is the southernmost intrusive in a belt of plutons in the northwestern part of the Candle quadrangle, in the extreme eastern part of the Seward Peninsula (fig. 2). The approximate center of the pluton and summit of Granite Mountain is occupied by a White-Alice communications station operated by ITT Arctic Services, which is scheduled for deactivation in 1976. An Air Force runway, adequate for mbst any type of aircraft, is localed on the west side of the mountain. Permission is required for its use. The nearest towns are Candle, $45 \mathrm{miles}$ northwest; Buckland, 40 miles north; and Koyuk, 40 miles south. The highest point is 2,344 feet in altitude. The terrain is rather subdued and mostly tundra covered; there are no trees. The Granite Mountain pluton is nearly circular and occupies about 30 square miles.

\section{General Setting}

The geology of cranite Mountain has been mapped and discussed by Miller and Elliott (1969), Miller (1970, 1972), Patton (1967), and Gault and others (1953).

The Granite Mountain pluton is a mid-Cretaceous intrusive complex that has intruded Jurassic(?) and early Cretaceous andesitic volcanic rocks located on the western edge of a late Mesozoic mobile Yukon-Koyukuk province (Patton, 1973). Tertiary and Quaternary flows are extensive in the area surrounding Granite Mountain.

Two small plutons satellitic to Granite Mountain are the fishhookshaped Quartz Creek pluton to the northwest and an unnamed stock near the northeastern part of the Granite Mountain pluton that occupies the headwaters of Peace River. The Quartz Creek pluton is of fine-grained biotitehornblende quartz monzonite, and the small unnamed stock is composed of several varieties of syenite. All three plutons have associated mineral occurrences. The Granite Mountain pluton and the unnamed stock are anomalously radioactive and are considered to be potential sources of uranium.

\section{Igneous Rock Types}

The alkaline intrusive complexes of west-central Alaska are typically highly potassic and subsilicic. The surrounding country rock is Lower cretaceous andesitic volcanic rock, and a contact aureole facies of hornfels extends outward for about $300 \mathrm{~m}$.

The Granite Mountain pluton is a zoned complex that has been divided into four units (Miller, 1972, p. 2118-2121). It consists of a core of equigranular quartz monzonites; an imner crescentric zone of massive to porphyritic monzonite and quartz monzonite; an outer crescentric zone of foyaite; and a unit of garnet-bearing nepheline syenite. Also, pseudoleucite porphyry as xenolithic-like blocks in the foyaite and as dikes in the andesite country rock suggests early separation of leucite during fractional crystallization of alkaline magma. 
The Quartz Creek pluton is predominantly fine grained biotite $h o r n-$ Llende quartz monzonite that crops out along Quartz Creek and near the headwaters of the Kiwalik River. The northeastern part of the pluton is extensively altered.

The stock on the north flank of granite Mountain at the head of Peace River is composed of several varieties of syenite. The most abundant are a pink, medium-grained hornblende-biotite variety and a pink, fine-to medium-grained, porphyritic syenite composed of over 90 percent perthitic Feldspar (Miller and Elliott, 1969, p. 12). Garnet-bearing nepheline syenite is also present. Anomalous quantities of metals and high radioactivity in the area make the stock particulariy interesting from the standpoint of uranium exploration.

\section{Economic Geology}

The mineralization at the Granite Mountain pluton offers strong encouragement to exploration for vein-type uranium deposits. Fourteen lode deposits containing one or more minerals of lead, silver, gold, zinc, copper, or tungsten, and about 20 gold deposits have been listed for the area (Cobb, 1972). Placer gold deposits have been worked on a small scale at several streams on the eastern and southern part of the mountain since the early 1900's. A little platinum was reported as a by-product. Description's of new metaliferous deposits and stream-sediment analyses have been published by the U.S. Geological Survey (Miller and Elliott, 1969; and Elliolt and Miller. 1969). Three areas were found to be of special interest and recommended for additional exploration by the authors: lead, zinc, and silver near Quartz Creek; molybdenum, bismuth, silver, copper, lead, and uranium deposits in the upper Peace River drainage; and a lead, zinc, and gold deposit at Bear Creek.

The Quartz Creek area contains numerous occurrences of argentiferous galena, sphalerite, pyrite, and arsenopyrite in an altered zone 18 miles long and 2 to 5 miles wide. The association of the sulphides with tourmaline is a striking feature. Stream-sediment samples yielded anomalous amounts of copper, antimony, and tin in addition to the metals already mentioned.

Anomalous concentrations of molybdenum, bismuth, gold, copper, and lead were found over a 2-square-mile area in the soils, stream sediments, and outcrops in the Peace River drainage basin. The metals are disseminated in a syenitic stock satellitic to the main Granite Mountain pluton. Pan concentrates collected during uranium investigations by the USris (cault and others, 1953) showed anomalously high concentrations of uranothorianite and a variety of other minerals, including galena, chalcopyrite, bornite, tetrahedrite, sphalerite, pyrite, and pyrrhotite. Gummite was also observed in some mineral grains. The source of the uranothorianite was not located, but west in fiault and others, 1953, p. 29-30) suggested the possibility of a lode at the head of Peace River.

\section{Previous Investigations for Radioactivity}

Ground investigations for radioactive materials were conducted in the eastern part of the Seward Peninsula by the USGS in the late 1950's (Gault and others, 1953). Significant amounts of radioactive minerals were found 
on the southern slope of cranite Mountain in placer concentrates from Sweepstakes and Ruby Creeks. Gault and others (1953, p. 1) described the findings:

1) signjficint content of radioactive materials was recognized in a few placer concentrates from Sweepstakes and Ruby Creeks in the northeastern part of the Seward Peninsula, Alaska, when old collections were scanned for radioactivity in the spring of 1945 .

The later field investiqations indicate that syenite is the only bedrock which has noticeallle radioactivity, and stream concentrates that were radioactives were obtained only from creeks containing syenite in the gravtis or flowing in areas underlain in part at least by the syenite. Crushed syenite samples from 14 localities show a content of radioactive material rangino from 0.001 to 0.013 percent equivalent uranium. The most radioactive unconcentrated material found is a 1 -inch pegmatite dike cutting the syenite. The syenite stock is pre-cretaceous and intrudes andesitic tuffs and flows that form the bedrock over much of the area.

Two radioactive minerals have been recognized from the photographic effects obtained on alpha-ray plates, and are tentatively identified as uraninitc-thorianite and hydrothorite. Almost all of the radioactive grains are uraninite-thorianite and only a few grains of hydrothorite were jdentified. Chemical analysis of a concentrate collected in 1917 from sweepstakes creek shows upproximately equal amounts of uranium and thorium, and together they form more than 80 percent of weight of the sample. Chemical analyses of 5 of the samples collected in 1945 indicate a uranium content of 0.008 to 2.17 percent. In the sample which has 2.17 percent uranium, beta counts show 14.20 percent equivalent uranium and the difference is believed to be thorjum.

The occurrence of uranium and thorium in the headwaters of the Peace River on the southeast side of Granite Mountain has been described by West (1953, p. 28-31). Reconnaissance investigations for uranium during 1947 and 1952 revealed uranothorianite and gunmite associated with copper sulfides, iron oxides, molybdenite, gold, silver, bismuth, and thorite in placers in a headwater tributary of the Peace River. Anomalous metal concentrations in strean sediments and outcrops occur over a 2-square-mile area underlain by a small satellitic stock of the Granite Mountain pluton (Miller and Ellioct, 1969, p. 12). The syenite locally contains purple fluorite. Concentrates from the placers contained between 0.2 and 0.8 percent eU, or about 10 times the ell of the average uranothorianite-bearing concentrates from other locations in the eastern part of the Seward Peninsula. The investigator of the Peace River locality concluded that the most probable source of the uranothorianite and gummite was a vein located in the rather restricted drainage area above the placer deposits. The evidence for a vein source is the sulfides associated with the uranium minerals disseminated within the granitic rock itself. Although metallic lodes are known to occur in the general area, no uranium ininerals were found in place.

Herreid (1965, p. 1 1 1 ) briefly visited the above mineralized area at the head of Peace River and obtained $350 \mathrm{ppm}$ lead from a panned sample, 
and copper, lead, and molybdenum from stream sediments downstream. Heavy hematite staining of the creek gravels was reported.

After radioactive minerals had been found on the southern side of Granite Mountain, the USGS continued investigations with a field study of the north side, in the headwaters of Quartz Creek (Gault and others, 1953, p. $(5-20)$ :

The area to the north of Granite Mountain has even more uranothorianite (uraninite-thorianite of Gault, Black, and Lyons, 1946; and Frondel and Fleischer, 1959, p. 7) than the sweepstakes creek area and in aduition carries uranium-bearing thorite(?).

The gravels of Quartz creek had formerly been mined for placer gold. The radiometric readings on the syenite bedrock was two to four times that of any of the surrounding rocks, and only the stream gravels derived from the syenite were radioactive. Tracing the radioactlve gravels upstream, uranothorianite and thorite(?) were found in wash on the bank of a guich in the headwaters of the south fork on Quartz creek. The heavy fraction of two samples contained 0.06 and 0.038 percent el ( $K$ illeen and White, 1953, p. 17). Concentrates from 21 stream gravel samples averaged 0.026 percent ell. Most of the radioactivity was attributed to uranothorianite and thorite, but radioactive zircon and sphene also contributed. The source of the uranium and thorium was thought to be either undiscovered veins or disseminations in the syenite of Granite Mounlain.

\section{DARBY MOUNTAIN AREA}

Location

The Darby Mountains are situated in the southeastern part of the Seward Peninsula in the eastern half of the Solomon quadrangle and in the southeastern corner of the Bendeleben quadrangle ( $($ igs. $2 \varepsilon 6)$. The range extends from Cape Darby on the south end of the peninsula between Golovnin Bay and Norton Bay to about $80 \mathrm{miles}$ northward where it merges with the Bendeleben Mountains. The low mountains in the southwest part of the area was called the Kwiktalik Mountains, but there is no distinct separation from the Darby Mountains.

The maximum altitude of the Darby Mountains is 3,169 feet. While this is a relatively low range composed mostly of rounded, tundra-covered mountains and hills, it has been glaciated and the higher ports display steepsided U-shaped valleys, cirques, and rock pinnacles.

There are no roads in the region, but short landing strips are present at the sma!l rative villages of Elim and Golovin on the coast at Golovnin and Norton Bays, respectively. A longer air strip and buildings at the abandoned FAA station at Moses Point were used as a base for the work during this investigation. Permission for its use is required from the Elim Natives.

The weather in the Darby Mountains is similar to that of much of the Seward Peninsula and is characterized by frequent fog, rain, and windstorms. The average annual rainfall at Nome is 17.88 inches and the average temperature is $26.10 \mathrm{~F}$. 


\section{freneral Geologic Setting}

The general geology of the Darby Mountains area has been described by Mendenhall (1901); Smith and Eakin (1911); West (1953); Miller and others (1972): Miller and Bunker (1975) and Herreid (1965). The northern part of the Darbys is also covered by Sainsbury's geologic map of the Bendeleben quadrangle (1974). The most complete coverage is the reconnaissance geologic map by Miller and others, and the following bedrock descriptions are largely derived from the report accompanying that map. The petrology has been discussed in coisiderable detail by Miller and Bunker (1975).

The core of the Darby Mountains consists predominantly of a Cretaceous pluton that extends 50 miles from near Cape Darby northward to the south side of Death Valley. The southwestern part of the Darby Mountains is composed largely of the Kachauik pluton, which is separated from the Darby pluton by a narrow ( $1-3$ miles) migmatic zone (fig. 6$)$. The plutons are bordered by Precambrian metasediments and Devonian carbonates.

The Darby Mountain piuton is a relatively homogeneous quartz monzonite, but Miller and 8 unker found indications of lateral zoning. Modes show a slight and gradual decrease in mafic minerals and plagioclase from south to north and a corresponding increase in K-feldspar and quartz.

Miller and Bunker (1975, p. I) have pointed out that the Darby plutons contain well above average amounts of $U$ and $T h(11.2 \mathrm{ppm}$ and $58.7 \mathrm{ppm}$, respectively) and the Kachauik pluton ranges from average to above average $\mathrm{U}$ and $\mathrm{Th}$ ( $5.7 \mathrm{ppm}$ and $22.5 \mathrm{ppm}$, respectively).

The most characteristic features of the Darby pluton are its uniform coarse-grained porphyritic texture, homogeneous composition, and relative abundance of magnetite and allanite. Lesser amounts of sphene, apatite, zircon, fluorite and rutile are present as accessory minerals.

The Kachauik pluton occupies about 205 square miles in the western part of the Darby Mountains. It is a composite intrusion. The west half is composed of granodiorite and quartz monzonite and the east half consists of a monzonite-syenite unit which has been subdivided into four subunits by Miller and others (1972).

A small stock on the western flank of the Darby Mountain about 6 miles north of the northern end of the Kachauik pluton is the Dry Canyon pluton. This intrusive body is of interest because it is composed of nepheline syenite and because of its highly anomalous radioactivity.

investigations by DGGS did not extend northward to the Windy Creek stock or the Bendeleben pluton. Their compositions and $U$ and Th contents reported earlier by Miller and Bunker (1975) indicated that time would be more profitably spent in the Darby Mountains.

The K-Ar dates of the plutons reported by Miller and Bunker (1975, p. 10) indicate a Late Cretaceous age of between 88 and $94 \mathrm{~m} . \mathrm{y}$. for the Darby pluton. The age of the Kachauik pluton is less certain. but the two dates reported are $86.1 \pm 3 \mathrm{~m} \cdot \mathrm{y}$. and $93.9 \pm 3 \mathrm{~m} \cdot \mathrm{y}$. 
The investigation by the Alaska State Division of fieological Survey was directed at determining the uranium potential of the plutons in the Darby Mountain area, and little attention was devoted to the surrounding metamorphic or sedimentary rocks. However, investigations of the potential of the basins or lowlands are needed. Little is known about the Cenozoic sediment in the lowlands on either side of the Darby Mountains. Tundra, muskeg, and lakes mask the sediments and no subsurface exploration has been done. Detailed petrographic and radioelement studies of the plutonic rocks are a part of this report prepared by Dr. R.B. Forbes and 8 . Jones.

A small interior basin west of the northern Darby Mountains is located in the upper Fish River lowlands (McCarthy's Marsh), in the south-central part of the Bendeleben quadrangle and the north-central part of the Solomon quadrangle. Nothing is known about the sediments under the Quaternary deposits, but the confined shape of the basin and the composition of the nearby bedrocks make it interesting to speculate on the possibility of uranium being concentraced in the sediments. The curving Bendeleben Mountains on the north, the Darby Mountains on the east, and unnamed hills to the south and west form a bowl-shaped basin about $20 \mathrm{miles}$ from east to west and 10 to 15 miles from north to south. The basin is drained by the Fish River, which flows southwest through a narrow valley.

West of the Kachauik Mountains the lowlands around Golovnin Lagoon and along the lower Niukluk River may have received sediments from the Darby and Kachauik Mountains. Although their presence is not known, nonmarine Tertiary sediments derived from the favorable source rocks may lie beneath the Quaternary cover and have a potential for uranium.

\section{Economic Geology}

The Darby Mountains have not been found to be mineral-rich, but numerous prospects and geochenical anomalies have been reported that suggest a potential for several metals.

The one mine that has produced is the Omilak mine, located in Precambrian marble on the west side of Omilak Mountain near the northern end of the Darby Mountains. It produced a few hundred tons of lead-silver ore prior to 1900 (Herreid, 1965). Additional lead and zinc occur at a prospect on Dry Creek south of the Omilak Mine.

Placer tin has been reported from alluvium on otter Creek on the east side of the northern end of the Darby Mountains (Herreid, 1965, p. 5). A lode gold prospect was explored in quartz-mica schist a half mile south of the otter creek tin placer. Placer gold prospecting has been conducted on several tributaries to the Tubutulik River.

Geochemica) stream-sediment sampling (Miller and others, 1971; Miller and Grybeck, 1973) has yielded highly anomalous copper, nickel, cobalt, chromium, manganese, iron, boron, scandium, and vanadium from the eastern side of the Darby Mountains. Gossan zones with high bismuth and molybdenum are present in the northern part of the range.

\section{Previous Investigations for Radioactivity}

As early as 1948 a reconnaissance for radioactive deposits revealed high uranium and thorium contents in the Darby and Kachauik plutons (West, 
1953). Ouring the course of the investigation 248 panned concentrates were collected from streams, beach gravels and slope wash. These were further concentrated in the laboratory. Anomalous (over 0.02 percent equivalent uranium) values were obtained over widespread areas, but principally in the northeastern part of the Darby pluton and the southwestern side of the Kachauik pluton. The radioactivity was essentially in minerals derived from felsic igneous racks. The most common radioactive minerals were sphene, allanite, hematite, and zircon. A uraniumbearing niobate mineral was found in slope wash near the shore on Golovnin Bay. Thorianite was identified near the head of kwiniuk River. Anomalous niobium was noted in the clear Creek-Vulcan Creek area.

More recent study of the Darby, Kachauik, and Bendeleben plutons by the U.S. Geological Survey (Miller and Bunker, 1975) has provided considerable data on the petrology and uranium and thorium contents. Results of the investigation showed compositional and textural differences indicating different sources for the three plutons and different average amounts of uranium and thorium.

Thirteen samples from the Darby Pluton contained above average amounts of $U$ and Th ( $11.2 \mathrm{ppm}$ and $58.7 \mathrm{ppm}$, respectively); the maximum values were $19.89 \mathrm{ppm} U$ and $33.75 \mathrm{ppm} \mathrm{Th}$. The Bendeleben pluton was found to contain normal amounts of $U$ and $T h(4.38 \mathrm{ppm}$ and 22.35 , respectively).

While the number of samples was too few to define the most anomalous areas for $U$ and $T h$, the authors concluded that the Darby pluton is similar to the Conway Granite in New Hampshire, which has been considered a pocential low-grade U-Th resource and is definitely a U-Th rich province.

\section{HOGATZA PLUTONIC BELT OF WEST-CENTRAL ALASKA}

The following discussion of the Hogatza Plutonic Belt is from Eakins (1975. D. $113-142)$.

Alkaline intrusive rocks occur in a belt extending 225 miles from the Seward Peninsula and Kotzebue Sound eastward to Hughes on the Koyukuk River. Patton (1970, p. 1; 1973, p. A4) applied the name Rogatza Plutonic Belt to this feature. The belt is roughly 20 miles wide and lies principally in the western part of the Candle quadrangle and the southern parts of the Selawik, Shungnak, and Hughes quadrangles (figs. D1, D2, D3). This region is in the northern part of the Yukon-Koyukuk province, named after the principal rivers. The compositions of the plutons and the associated radioactivity anomalies make this one of the most interesting regions in the state for uranium-polential study.

The mountains and hills in the region are generally low and rounded and largely covered by $50 \mathrm{il}$ and vegetation. Weathering has reduced most outcrops to rubble, and good exposures of the bedrock are scarce. Summits reach a maximum altitude of 3,300 feet in the Selawik Hills and 4,050 in the Zane Hills. Elsewhere the altitudes are less than 3,000 feet. The lowlands north of the plutonic belt are swampy, lake dotted, and masked by alluvium and morainal deposits.

The region lies approximately on the boundary between the zones of continuous and discontinuous permafrost. The average annual temperature 


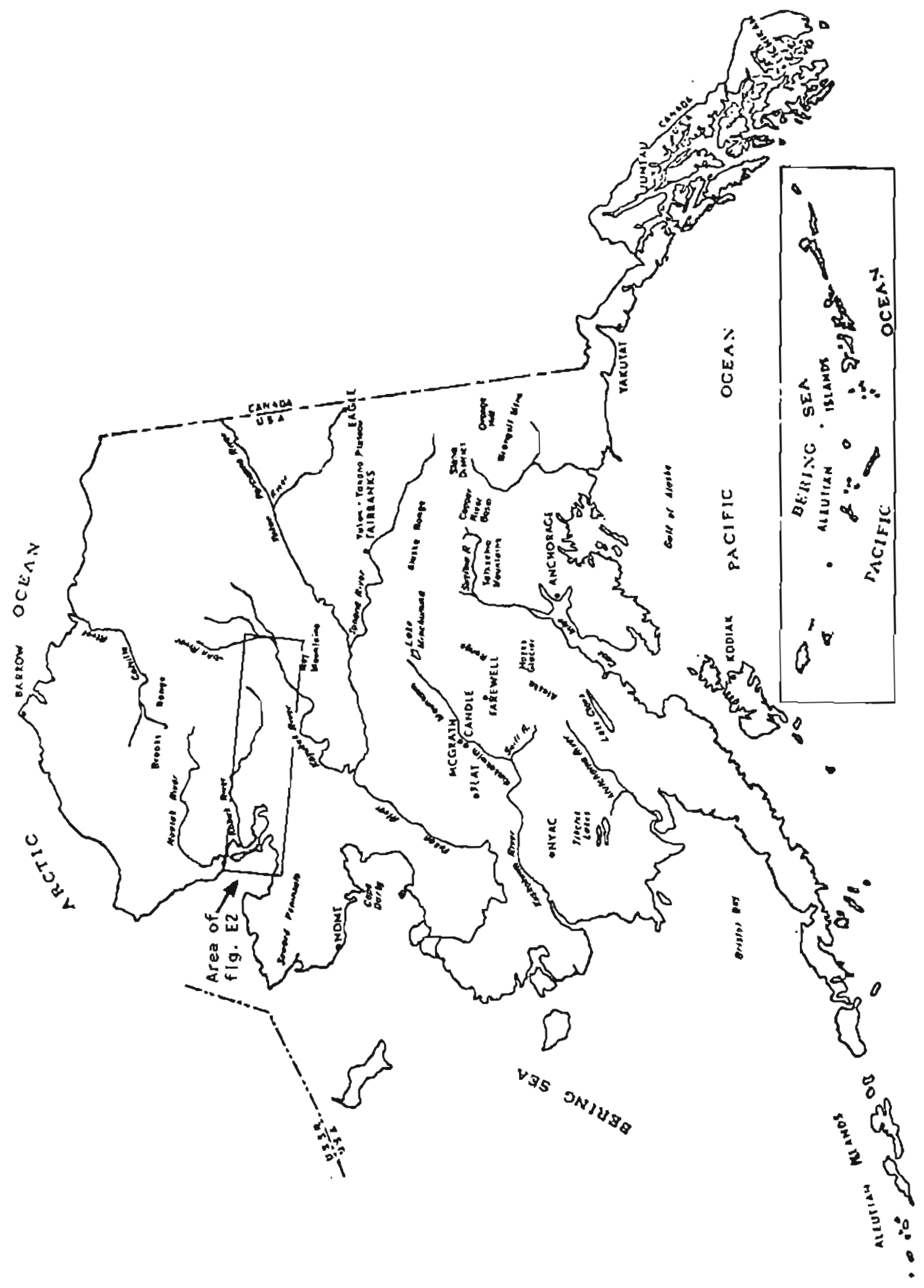

Figure El. Location of the Hogatza plutonic belt. 

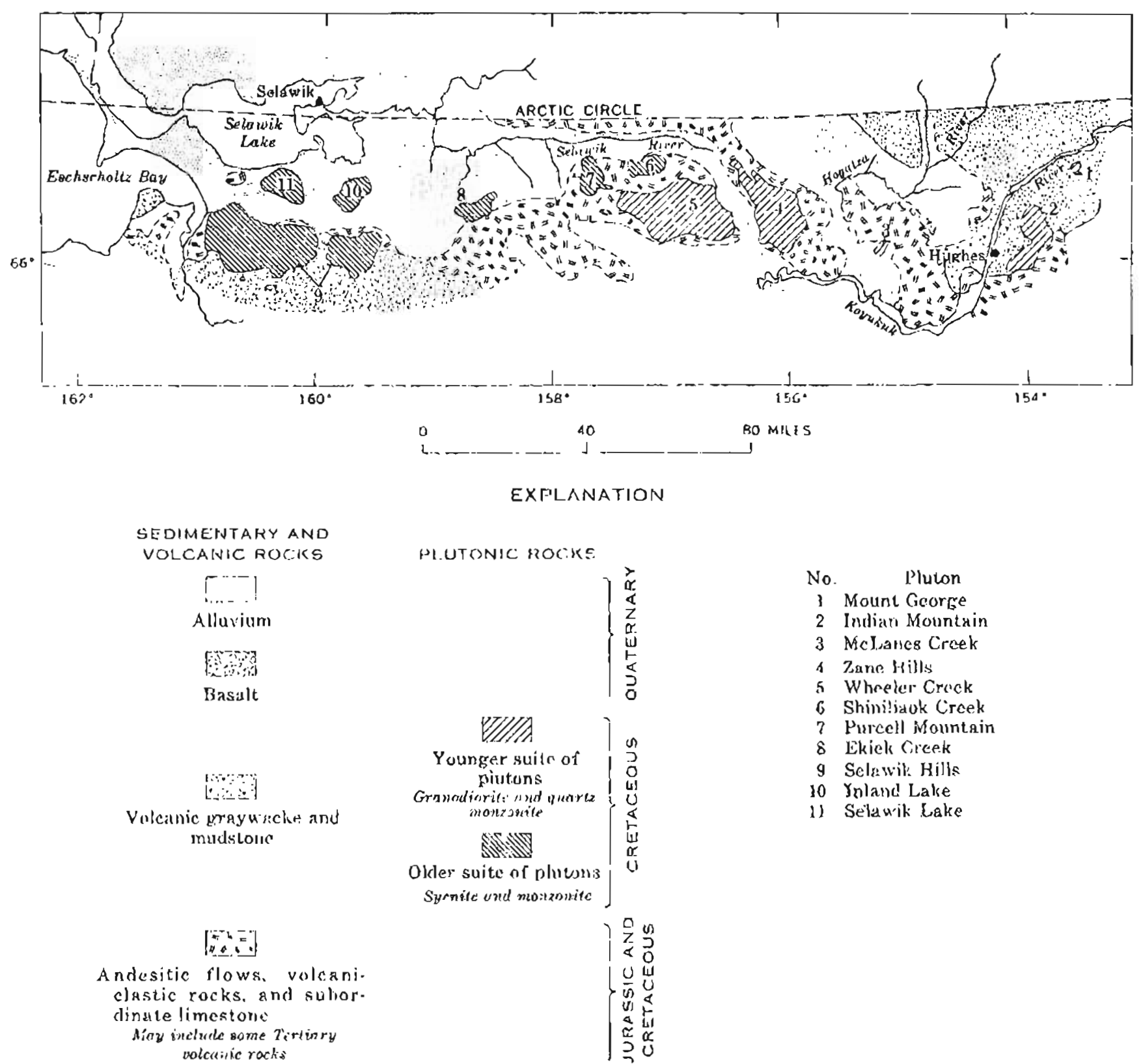

Figure E2. Generalized geologic map of west-central Alaska, showing location of plutons. Source: Miller, Patton, and Lanphere, 1966. 

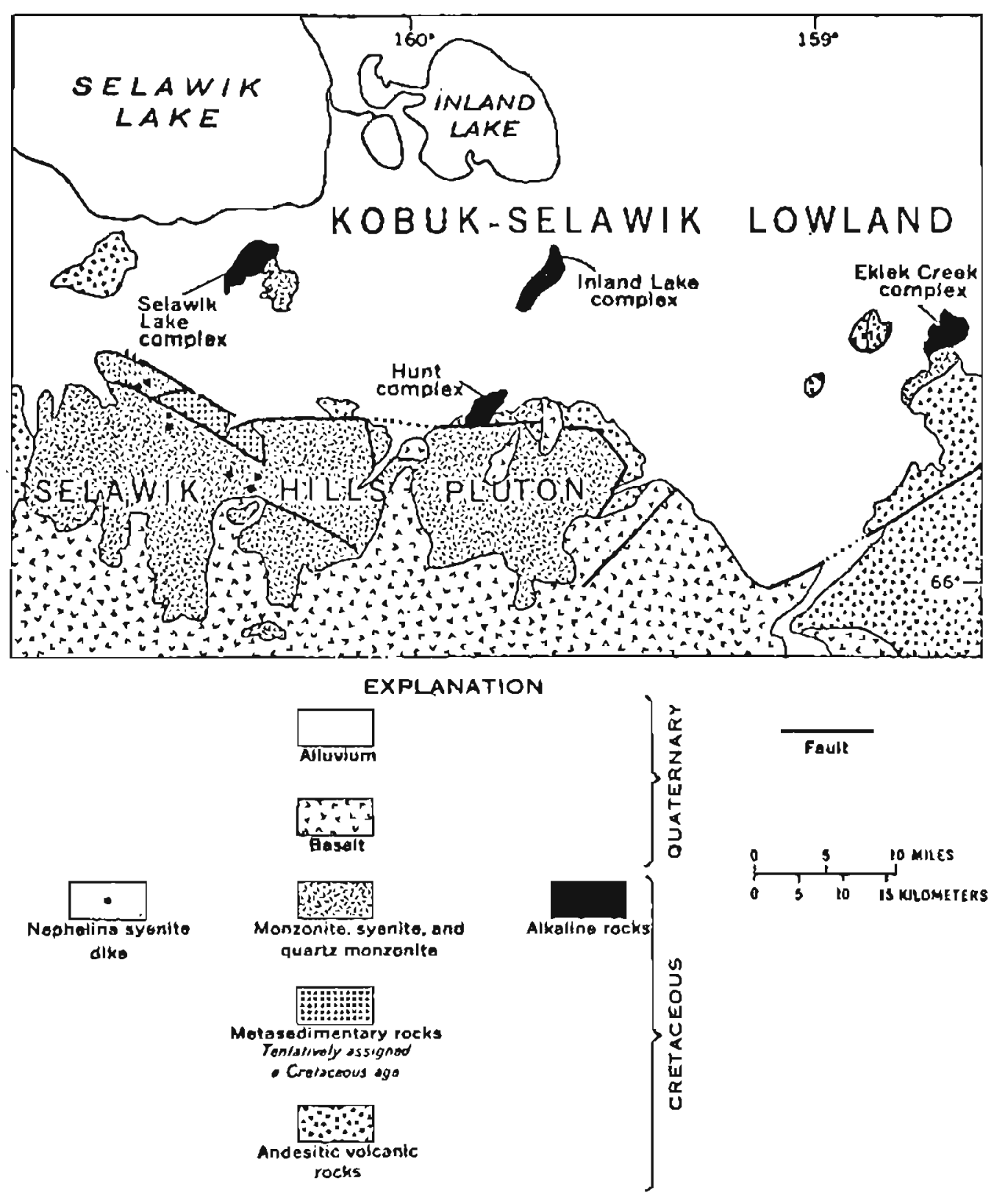

Figure E3. Generalized geologic map of the Kobuk-Selawik lowland and Selawik Hills (after Patton and Miller, 1968). Source: Miller, 1972. 
at Kotzebue is $20.7^{\circ} \mathrm{F}$. The climate is arid; the average annual precipitation recorded for Kotzebue is 8.18 inches. Small, widely spaced settlements exist at Hughes, Hog River. Gabolio and Selawik.

The region is relatively inaccessible and has been little prospected. However, geologic mapping, geochemical sampling, and radionetric surveys by the U.S. Geological Survey since about 1966 have revealed interesting possibilities for base metals and uranium in the intrusive rocks. Little is known about the sediments underlying the lowlands flanking the uplift or their potential for petroleum and uranium.

Early reconnaissance geology and mapping in the region was done by Smith (1913) and Eakin (1916). Patton (1973) divided the sediments into three general sequences: 1) a Lower Cretaceous volcanic-mudstone sequence; 2) a younger sequence of Lower to Upper Cretaceous age, which was subdivided into four units; and 3) an Upper Cretaceous-lower Tertiary sequence. Because of the poor exposures, the exact stratigraphic relationships of the three sequences are not clear.

The oldest rocks of the basin are Lower Cretaceous in age and consist mostly of volcaniclastics, including lithic tuffs, breccias, conglomerates, and tuffaceous graywacke and mudstone which are believed to underlie the entire province. The thickness near Hughes is 5,000 feet, but the total may be several times this amount. Potassium-argon ages ranges from $134 \pm$ $5 \mathrm{nl} \cdot \mathrm{y} \cdot 10117+4.3 \mathrm{~m} \cdot \mathrm{y}$.

Lower and Upper Cretaceous rocks are present along the northern border and northeastern part of the province. These were referred to by earlier geologists as the Berguan Group (Schrader, 1904; Martin, 1926; Imlay and Reeside, 1954). Patton, however, abandoned the name and divided the sequence into four units:

(1) Volcanic graywacke and mudstone, to 5,000 feet thick. It contains an abundance of feldspar, some lithic tuff, and carbonized plant debris.

(2) Calcareous graywacke and mudstone--possibly as much as 5,000 feet of shallow-water calcareous graywacke and mudstone. The sediments coarsen westward, where they become conglomeratic and coal-bearing.

(3) Sandstone, siltstone, shale, and coal. Portions of this unit may be of interest to uranium potential studies, but its distribution is restricted to the lower Yukon-Koyukuk province.

(4) Marginal marine trough deposits. Nonmarine quartz conglomerate can be traced by scatiered outcrops for about 450 miles along the northern and northeastern margins of the Yukon-Koyukuk province. The thickness was found to be 3,000 feet along the lower Kobuk River. The unit is described as principally a quartz conglomerate, but it contains minor amounts of quartz sandstone, shale, thin bituminous coal beds, and ash-fall tuffs in the northern part of the Selawik quadrangle.

Upper Cretaceous and Lower Tertiary rocks include felsic extrusive and hypabyssal racks. The flows are as much as 2,000 feet thick. The intrusives consist of swarms of dikes, sills, and plugs that cut the older cretaceous volcanic and sedimentary rocks. 
Poorly consolidated nonmarine coal-bearing beds of Tertiary age have been found at two localities in the northwestern part of the province. Sandstone and gravel are exposed in a 30-foot bluff on the Mangoak River in the Selawik lowland (Patton and Miller, 1968); iignitic coal was found at the base of the bluff. A 2-foot seam of coal has been reported from a silt bluff near Elephant Point on Kotzebue sound. Both deposits are probably confined to small structural and topographic basins or faldt zones.

Petroleum companies have done limited work in the province in an effort to determine the petroleum possibilities. Subsurface data are not available from industry, but it is rumored that seismic surveys indicate several thousand feet of Tertiary and Mesozoic sediments with a good potential for oil are present in Kotzebue Sound. Standard Dil of California is preparing to drill three wells in the area in a joint venture with the Northwest Area Native Association, Inc.

The unexplored Selawik Basin immediately north of the belt of alkalic plutons offers a setting warranting subsurface work to determine if possible uranium host rocks are present. The south flank of the pluton belt is covered by Tertiary and Quaternary volcanics and appears less likely to have suitable sediments.

The Pah River Flats is a 20 - by 30-mile topographic depression bounded by the Lockwood Sills on the north, the Kokhila Hills on the east, and by the Babantaltlin Hills on the southeast. The hills are underlain by Cretacenus graywacke and mudstone and Jurassic and Cretaceous volcanics. The west and southwest edges of the depression are bordered by the Zane Hills pluton. which has yielded showings of copper, silver, gold, and molyodenum, and anomalous radioactivity. Border phases of the pluton near Caribou Mountain show radioactivity five to 10 times the background radioactivity of the rest of the pluton, and $20 \mathrm{ppm}$ uranium on analysis (Miller and Ferrians, 1968. p. 9).

The central part of the Pah River Flats is occupied by innumerable small lakes and muskeg. The saucer shape and poor drainage of this basin, which is adjacent to possible source rocks, suggest a favorable site for uraniuin concentration in sediments. However, available geologic maps of the area do not indicate the presence of a suitable host rock and drilling will be required to determine if any Late Cretaceous or Tertiary sandstones are present beneath the surficial deposits. The village of Hogatza, a few miles south of Pah River flats, could serve as a base for exploration.

Plutonic Rocks

The 225-mile-long pluton belt extends from the eastern edge of the Seward Peninsula eastward to a point about 15 miles east of Hughes on the Koyukuk River. The belt trends east-west along the Hogatza uplift in the southern parts of the Selawik, Shungnak, and Hughes quadrangles, and trends north-south in the western half of the Candle quadrangle. The main part of the uplift borders the south side of the Selawik basin and the Pah River Flats.

Sixteen separate plutons ranging from 3 to 350 square miles have been described and mapped (Miller and others, 1966, 1970; Patton, 1967; Patton 
and Miller, 1966, 1968; and Miller, Patton, and Lanphere, 1966). The largest (table DI) is the Selawik Hills pluton, which is 40 miles long and averages roughly $10 \mathrm{milles}$ in width. The aggregate exposed area of the plutons is about 1,200 square miles. Portions are overiain by volcanic flows and welded tuffs of Late Cretaceous. Tertiary, or Quaternary ages so that the actual size of the pluton is greater than the outcroppings.

The alkaline nature of the plutonic rocks is discussed by Miller (1972, p. 2122):

The field and analytical data show that the alkaline rocks of western Alaska are epizonal plutonic rocks that are highly undersaturated in silica and rich in alkalis. Total alkali content ranges from a 1.3 of 5.5 percent in biot.te lyroxenite to 17.9 percent in kalsilite-bearing juvite. These are not peralkaline rocks-with one exception, the molar ratio of total alkalis to aluminum is less than one.

Many chemical cha:actexistics of the alkaline rocks of western Alaska and Cape Dezhnev, while illustrating the alkaline nature of the rocks, are similar to those found in many alkaline-rock provinces. the western Alaska alkaline suite is unusual, however, in its high $\mathrm{K}_{2} \mathrm{O}$ content and high $\mathrm{K}_{2} \mathrm{O} / \mathrm{Na}_{2} \mathrm{O}$ ratio. $\mathrm{K}_{2} \mathrm{O}$ is over 6 percent by weight in 14 out of 22 analyzed samples and is as high as 16.6 percent.

Table Dl. Sizes of the plutons in the Hogatza pluton belt, west-central Alaska

Name

Area

$\left(\right.$ (mi ${ }^{2}$. $)$

Late Cretaceous Suite

Zane Hills pluton

Wheeler Creek pluton

271

Indian Mountain pluton

Mt. George pluton

Mclanes Creek pluton

Total

550

Mid-Cretaceous Suite

Shiniliaok Creek pluton 30

Purcell Mountain pluton 40

Hawk River slock

40

Ekiek Creek Complex

Selawik Hills pluton

354

Hunt Complex

Inland Lake Complex

Selawik Lake Complex

Hunter Creek pluton

165

Granite Mountain pluton

27

Quartz Creek pluton

$\frac{3}{653}$

Totals for both suites

$\overline{1,203}$ 
The alkaline character, wide tistribution, and reports of anomalous radinactivity of the plutons in the Honatza plucon belt suggest a highly favorable region for uranium investigations, especially in light of the recent development of large uranium reserves in alaskite at the Rossing deposit in southwestern Africa. While the entire belt warrants careful study, the alaskite occurrences may be of particular incerest, and a description of the Wheeler Creck pluton alaskite is quoted from Miller (1970, po. 101 and 103):

Alaskite of the wheeler Creek pluton--Conse-grained alaskite underlies the west end of the whecler creek pluton and intrudes rocks ranging from lower crotacrous andesitic volcanics to the upper Cretaceous dacitic hypabussal rocks. Alaskite outcrops are characterjzed by rounded pink-colored hill.s with littlc veqetation and a mant le of grus. The alaskite itcelf is characterjzed megascopically by large (up to $1 \mathrm{~cm}$ ) bluk smoky guartz anhedra in a setting of pink feldspar anhedra. The rock is characteristically coarse-grained with an allotriomorphic granular texture. The abundance of the smoky quartz distinguishes this unit from the minor alaskite and aplite dikes that Jocally cut the zane Hills pluton. The rock is genorally a true alaskite, with less than 1. percent mafic minerals, although locally the mafic content. reaches as much as 8 percent near thr contact and in the diaskjte dikes cutting the quartz monzonito-granodiorite to the east.

Alaskite is present in other plutons in the Hogatza belt either as a major rock type or in dikes. The southern and western parts of the Selawik Hills pluton has been mapped as predominanlly quartz monzonite and alaskite (Patton and Miller, 1968). The rest is largely syenite and monzonite. The composition, large size, and radioactivity anomalies of the Selawik Hills pluton make this an attractive area to explore for vein or Rossing-type uranium deposits. The present of purple fluorite associated with pulaskite and perthosite in the alkaline complexes in the Selawik lowlands (Miller, 1970, p. 46) may also be an indication of anomalous radioactivity. The possibility that this large pluton and others in the pluton belt have contributed significant amounts of uranium to concealed sediments in the lowlands warrants careful investigation.

\section{Structure}

The northern Yukon-koyukuk province is in a highly mobile region that was subjected to repeated magmatisn during Cretacenus and early Tertiary times. The Hogatza uplift extends from the Seward Peninsula and follows the east-west grain of the region. The Kobuk fault (or trench) trends along the northern border of the province. Small faults are visible in most bedrock exposures. While the sedimentary rocks are moderately to strongly deformed they have not been regionally metamorphosed.

The Kobuk-Selawik Lowlands is a major feature of the region, but aeromagnetic profiles suggest that igneous rocks are at shallow depth and it seems unlikely that Cretaceous or Tertiary sedimentary rocks are very thick beneath the Quaternary surficial deposits. Compilation of aravity surveys in northwestern Alaska by D.F. Barnes of the USGS shows a belt of gravity 
highs that extends through the Selawik Basin and militates "against the presence of the sedimentary basin postulated in preliminary petroleum investigations" (U.S. Geol. Survey, 1967, p. A9l). Farther west, however, cretaceous and younger sediments as much as 10,000 feet thick may underlie Kotzebue Sound (Patton, 1970, p. 1).

Economic Geology

Because certain mineral assemblages may be indicative of favorable environments for uranium, brief summaries of known mineralization are described below by areas. The areas will be mentioned in sequence, beginning at the western end of the belt.

Selawik Hills Pluton

The Selawik Hills pluton extends 45 miles east-west and underlies most of the Selawik Hills south of the Selawik Lowland. It consists principally of monzonite and syenite and is exposed over an area of about 350 square miles. The only information on the economic geology is that provided by stream-sediment sampling (Elliott and Miller, 1969, p. 6):

Many of the samples (1-38) from several small streams on the north flank of the Selawik Hills have slightly anomalous concentrations of lead (18 sampless with $70 \mathrm{ppm}$ and 6 samples with $100 \mathrm{ppm}$ ); and one sample, locality 32 , contained $300 \mathrm{ppm} l \mathrm{lead}, 200 \mathrm{ppm}$ zinc, and $3 \mathrm{ppm}$ silver. At bedrock locality $x$, near sediment locality 32 , minor amounts of disseminated gilena, sphaleritt, and pyrite were noted in quarti-calcite veins and in pink syenite. Composite grab samples of the sulfide-bearing rock contained up to 2 percent lead and up to 1 percent zinc, but the extent of the mineralized area could not be determined due to poor exposure.

Beryllium was detected in concentrations of 10 and $15 \mathrm{ppm}$ in four sediment samples $(44,45,48,51)$. from small streams on either side of the ridgo south of Clem Mountain. One sediment sample (59) from Hunter Creek, just above the Left Fork, contained 50 ppm tungsten and $30 \mathrm{ppm}$ molybdenum.

Abnormal radioactivity of phonolite, fluorite-bearing nepheline syenite, syenite, and trachyte was reported in the Selawik Hills pluton (Miller, 1968, table 4). The high radioactivity of the rocks is probably due in part at least to the high $\mathrm{K}_{2} \mathrm{O}$ content, which ranges from 4.8 to 8.4 percent and averages 6.2 percent (Miller, 1970, p. 28).

Kobuk-Selawik Lowlands Pluton

Two poorly exposed alkaline complexes crop out as low hills between the Selawik Hills and Selawik Lake (fig. D3). These are designated as the

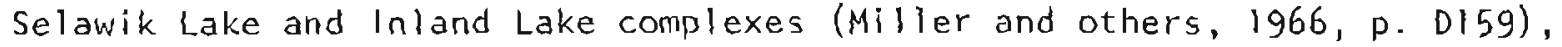
and are 7 and 12 square miles in area, respectively. The complexes consist of a variety of unusual alkaline rock types. Chemical analyses of the rocks show that lead, strontium, lanthanum, and arsenic are relatively high (Miller, 1970, p. 57). Unusual amounts of fluorine and zirconium are also present: up to 0.57 percent $F$ and 0.20 percent $2 \mathrm{rO}_{6}$. The only information on the 
economic geology is derived from two stream-sediment samples from the wost side of the selawik Lake pluton. One sample produced slightly anomalous cobalt (50 ppm) and copper (70 ppm) (Elliott and Miller, 1969, n. 10). Anomalous radinactivity of the piutons was reported from an aerial radiometric survey (Miller and Anderson, 1969).

Ekiek Creck Comole:x

The Ekiek Creek pluton is a small intrusive, about 5 square miles in area, located atout halfway between the Selawik Ilills and the Purcell Mountains (fig. D3). It contains a wide variecy of alkaline rocks. Two stream-sedimnt samples from the east side of the pluton did not show any anomalous metal values, but the aerial radiometric survey showed the northern end of the pliston to be anomalous ( 600 counts per second).

\section{Shiniliaok Creek Pluton}

The Shiniliaok creek pluton occupies about 30 square miles in the north-central part of the Purcell Mountains (fig. D2). It is composed chiefly of medium-grained monzonite and syenodiorite. Tourmaline occurs as a widespread accessory and as massive voins in fault zones (Miller, 1970, p. 39). Little is avoilable concerning the economic geology. A fow widely spaced stream-sediment samples did not yield significant anomalics (Miller, 1969).

Zane Hills Pluton

The Zane Hills pluton is 180 square miles in aren and forms a large part of the Zane Hills. The settlement of Hogatza (Hog River) is located on the east flank of the Zane Hills. The highest point in the area is Cone Mountain, 4,053 feet high. Granodiorite constitutes about 9 percent of the pluton;monzonite and quartz monzonite compose most of the rest. Alaskite and aplite dikes are common. Placer gold mining began at Bear Creek, on the east side of the pluton, in the early 1900's. A gold dredge was installed in 1957 and operated until 1975; it accounted for a substantial part of the state's gold producrion during that period. Unpublished reports indicate that cassiterite and platinum were also found in the Bear Creck placers. Bedrock and stream-sediment sampling (Miller and Ferrians, 1968, p. 6-10; Miller, 1969) revealed mineralization in the Zane Hilis piuton at several locations. Massive pyrite is associated with silver and gold near the north end of the pluton. Zinc and molybdenum anomalies were also found nearby. Uphill from the Hogatza placer mine on Bear Creek, sediment samples were found to contain anomalous amounts of silver, bismuth, copper, and molybdenum. Two strongly anomalous areas of radioactivity were found in quartz monzonite: on the east side of Caribou Mountain and at the southern end of the pluton. The radiometric anomalies of these border phases were $f$ ive to 10 times as high as readings over most of the pluton.

\section{Purcell Mountain Pluton}

The Purcell Mountain pluton is chiefly quartz monzonite and crops out over a 40-square-mile area in the Purcoll Mountains. The on iy known mineral deposit is a gold placer mine on Shovel Creek on the northwest slope of 
Purcell Mountain. The mine was worked for about 10 years during the 1950 's and 1960's, but the production is unknown. The gold may have been derived from quartz-tourmaline-sulfide veins near a contact between the quartz monzonite pluton and andesitic volcanics (Cobb, 1972, p. 35 ).

\section{Hawk River Pluton}

The Hawk River plutcn, lacated 6 miles southeast of Purcell Mountain peak and about 2 miles southwest of the Wheeler Creek pluton, occupies 5 square miles. The small Hawk River stock consisis of olivine-bearing monzonite cut by an east-west-trending fault (Miller, 1970, p. 38). A quartzrich zone 6-1/2 miles long by $1-1 / 2$ miles wide trends northeast between the Hawk River, iurcell Mountain, and Wheeler Creek plutons. Grab samples from this zone contained anomalous values of copper, arsenic, lead, and zinc. Stream sediments indicated anomalous lead, copper, and silver (Miller and Ferrians, 1968, p. 10-!1).

Wheeler Creek Pluton

The Wheeler creek pluton underlies about 271 square miles of the Purcell Mountains. It is separated from the Zane Hills on the east by a 6mile-wide valley. Most of the pluton is composed of porphyritic quartz monzonite and granodiorite. The west end of the pluton consists of coarsegrained alaskite which is characterized by smoky quartz. The alaskite body is about 36 square miles in outcrop area. Streani-sediment sample analyses did not reveal any mineralized areas (Miller, 1969), and no ore deposits are known.

Previous Radioactivity Investigations

Anomalous radioactivity has been reported from the plutons in westcentral Alaska as a result of aerial and ground radiometric surveys, chemical analyses of the intrusive rocks, and testing of panned concentrates from stream graveis. High background counts can be expected over much of the pluton belt because of the unusually high potassium content of the rocks; however, uranium and thorlum minerals have definitely been identified. Favorable mineral assembleges also are suggestive of possible vein-type uranium deposits.

An airborne radioactivity survey in conjunction with an aerial magnetometer survey covered 1,320 square miles of the southern Kobuk-Selawik lowland (Miller and Anderson, 1969). The background over the lowlands and volcanic rocks was generally less than 100 cps (counts per second). The highest count (700 cps) was obtained over granitic rocks at the northern tip of the Selawik Hills pluton. Counts of 500 cps were obtained over both the nepheline syenite of the Selawik Lake, $\ln l$ and Lake, and Ekiek Creek plutons--a figure five to 10 times higher than that of the surrounding terrain.

Another aerial radioactivity survey was a single flight line made from near Kiwalik south of Kotzebue Sound eastward 80 miles, which covered the southern edge of the above-mentioned survey and traversed the northern part of the Selawik Hills. The survey was sponsored by the USGS. The maximum count reported was 1,600 cps, registered over the northern tip of the Selawik Hills pluton. Other anomalies of 800 to $1,000 \mathrm{cpm}$ were obtained along with flight line. The background was between 300 and $400 \mathrm{cpm}$. 
Two areas of the Zane Hills pluton near Carihou Mountain have been found to have strong radioactivity anomalies. These areas have been described and mapped by Miller and Ferrians (1968, p. 9-10):

Bordor phases of the 7iane llilis pluton in two axeas along the routhrastirn margin of the pluton show anomalous radioactivityrive to tor times the background radingctivity of the rest of the piutrn. Those border phasns are composed of medium- to coarsegrained, trachytoid to gneissic, hornhlende-biotite quartz monzonite and monzonite readily distinguishablo in the field from the typical massive, granitic-textured granodioxito of the rest of the piuton. An arilysis of porphyzitic gunrtz monzonite from this border phase shows $20 \mathrm{ppm}$ of uresnium. This is five to six times more than the published averages for rocks of this composition (Smith, 1963, p. 407). Examination of thin scctions of this rock shows that hiotite and hormblende contain numerous inclusions surrounded by pleochroic halos indicative of radinactivity. Some of these halos are obviously around zircon exystals, but other much more intense halos arc around grains of a colorless to faintly yollow, isotropic mineral of high relief. A thin section of this porphyritic quarta monzonite was exposed to a thermal neutron buam in a reactor in order to cause the fission of $u^{235}$. The fission cucnts wcre recorded in a piece of Iexan which covered the section. Later, etching of the lexan showed the anomalously occurring uranium in the samplo to be associated with the colorless isotropio mineral.

Although the uranim-bearing mineral is only a minor constituent in the samples studied, it may be more abundant olsehwexe in the radioactive horder phase-possibly in amounts large enough to be important economically, or other uranium minerals may be prosent. A panned concentrate collected in 1964 from caribou creck on the southeastern side of the lane Hills contained 200 ppm of thorium, which was probably derjved from this more radioactive border phase of the pluton.

COPPER RIVER BASIN-CHITINA VALLEY

Location

The Copper River basin is a topographic and structural basin in south-central Alaska. It is bounded on the north by hills along the southern flank of the Alaska Range, the Wrangell Mountains on the east, the Chugach Mountains on the south, and the Talkeetna Mountains on the west (fig. 16). Fringe areas considered include the upper end of Matanuska Valley, the Gulkana upland along the southern flank of the Alaska Range, and the Chitina River Valley, located between the Wrangell and Chugach Mountains. The Copper River basin is roughly about 80 miles across from east to west and is mostly within the Valdez and rulkana quadrangles of the U.S. Geological Survey topographic map series. The eastern and southern parts of the Copper River area are accessible by the Richardson, Glenn, and Edgerton Highways. The Denali Highway crosses the northern end. Small landing fields are located at a number of the settlements along the highways.

The early history and development of the region are closely tied to the fanous Kennecott Copper Corporation mines located in the Wrangell Mountains near McCarthy, on the north side of the Chitina Valley. Recent interest in the region has been directed towards determining the petroleum possibilities of the Mesozoic section of the Copper River basin. 
The altitude of the lowlands in the basin is between 2,000 and 3,000 feet above sea level. It is occupied by numerous lakes and is drained principally by the Copper River, which has its out?et in the rulf of Alaska; drainage is porly developed over a large area, and ground travel is difficult. The mountains bordering the basin are high and rugged and have been the source of many glaciers which have supplied drift coverage in the lowlands and creaced the innumerable small glacial lakes. Glaciers are still abundant in the Alaska, Wrangeil, and Chugach ranges. Mit. Blackburn and Mt. Sanford in the Wrangclis are both over 16,000 feet high. The 14,000-fooi-high Mt. Wrangell volcano emits smoke and steam.

The climate is much more arid than in the coastal regions: at Copper River townsite the average annual precipitation is barely 9 inches. The region is in the belt of discontinuous permafrost.

The geology of portions of the province has been described and mapped by Chapin (1918), Mertie (1927), Moffit (1938), Capps (1940), and Andreason and others (1964).

\section{Sedimentary Rocks}

The Copper River Basin is Cenozoic but the southern part includes a thick sequence of Mesozoic sediments of the Matanuska geosyncline (13ayne, 1955). The deepest test wel! in the basin was drilled to 8,837 feet and was still in Jurassic sediments. Most of the units have been dated approximately by marine fossils but complicated structure, rapid changes in lithology and chicknesses, and extensive erosion make some corrclations doubtful and confusing. Although most of the pro-Tertiary units are clearly marine or marginal marine, there are several thick sandstone and conglomerate sections of interest exposed in the Chitina valley area and to a lesser extent in the southwestern part of the basin in and north of the upper Matanuska Valley. The stratigraphic units are remnants of the once-extensive deposits which in part extend beneath the nuaternary cover in the Copper River basin. Some of the Mesozoic sandstones are arkosic and tuffaceous, and contain a small amount of carbonaceous material, discussed in the following summary of the stratigraphy. The Tertiary beds are of continental origin and are probably the most interesting rocks in the region with respect to uranium potential, although outcrops are very linited. reneralized stratigraphic sections for the east and west sides of the basin are shown in figures 04 and 05 .

Descriptions of much of the stratigraphy is necessarily based upon work in the upper part of the Chitina valley, as much as 100 miles from the Copper River basin lowlands, where outcrops are available for examination and where mining has been important. firantz (1965) produced a detailed map and cross sections of the western flank of the basin that show subdivisions of the Mesozoic units and illustrate the complex structure along the eastern side of the Talkeetna Mountains. The intrusive rocks and metamorphic complexes in the northern and northwestern parts of the Copper River basin have most recently been mapred by T.E. Smith $(1973, p .3-6)$. Limestone is dolomitic in mart and the host for the massive copper deposits near Kennirott, where it is up to 3,000 feet 


\begin{tabular}{|c|c|c|c|}
\hline LEYSE & Epnch & formation & Thickness \\
\hline $\begin{array}{l}L \\
\stackrel{U}{\Xi}\end{array}$ & Mlocene-Pl locene & Wrangell Lava & $0-5,000^{\prime}$ \\
\hline 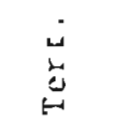 & Mrocene & Fredertka Fm. & $2,000^{\circ}$ \\
\hline \multirow{4}{*}{ 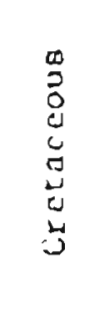 } & \multirow{4}{*}{$\begin{array}{c}\text { Upper } \\
\text { Cretaceous }\end{array}$} & $\mathrm{SacCa} 11 \mathrm{Fm}$ & $2,500^{\circ}$ \\
\hline & & Chititu Fr. & $5,500^{\circ}$ \\
\hline & & Schulcz $F_{m}$ & $225^{\prime}$ \\
\hline & & Moonshtne Creek Fm. & $3,500^{\prime}$ \\
\hline \multirow{4}{*}{ 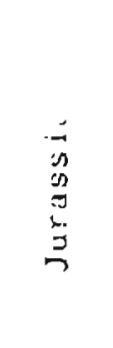 } & Lower Cretaceous & Kennecott Fm. & $1, .500^{\prime}$ \\
\hline & \multirow{3}{*}{$\begin{array}{l}\text { Upper Jurassic } \\
\text { Middle and } \\
\text { Upper Jurassic }\end{array}$} & Root Glacler Fm. & $0-4,00 n^{\prime}$ \\
\hline & & Nazina Mountain Fm. & $0-1,400^{\prime}$ \\
\hline & & Kotsina Conglomerate & $2,000-2,500^{\prime}$ \\
\hline \multirow{5}{*}{ 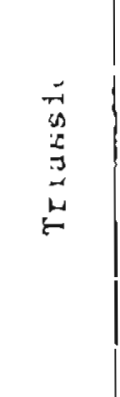 } & \multirow{3}{*}{$\begin{array}{l}\text { Lower Iarassic } \\
\text { Upr. Triass. \& Lwr: } \\
\text { Jurasstc }\end{array}$} & Lube Creek & $300^{\prime}$ \\
\hline & & McCarthy Shale & $1,500-3,000^{\circ}$ \\
\hline & & Nizina Limestone & $1.100^{\prime}$ \\
\hline & Upr. Triasslc & Chitistone Limestone & $1,900^{\prime}$ \\
\hline & $\begin{array}{l}\text { Mid. d/or Upr. } \\
\text { Trtassic }\end{array}$ & N1kola1 Greenstone & $5,000^{\prime}$ \\
\hline \multirow{2}{*}{ 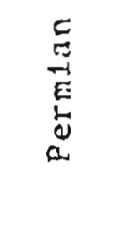 } & \multirow[t]{2}{*}{ Lower Permian } & Honsen Creek Fm. & $900^{\circ}$ \\
\hline & & Station Creek Fm. & $6,500^{\circ}$ \\
\hline
\end{tabular}

Base not exposed

Flgure E4. Generallzed stratigraphic section--east side, Copper river Basin (Chitina Valley area), Alagka. (Source: Nlaska Geological Soctety

Stratigraphic Comnttee, 1969-70.) 


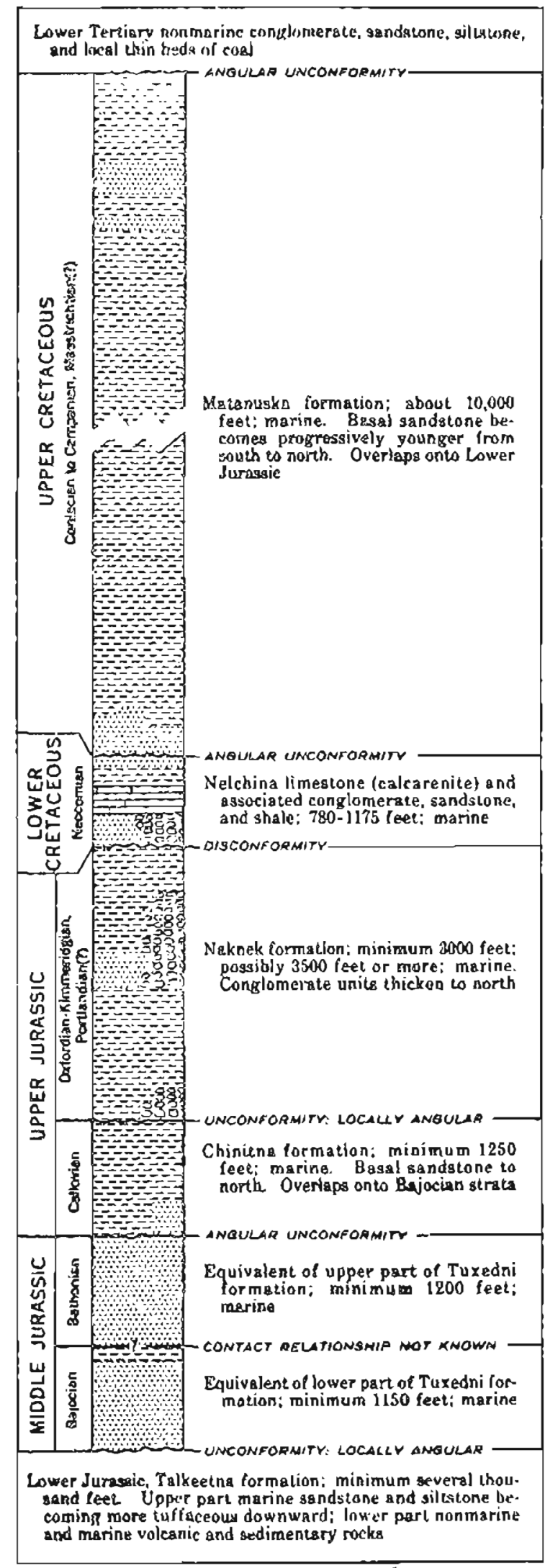

Figure E5. Generalized stratigraphic section--west side, Copper River basin (Nelchina area), Alaska. Source: Grantz, 1965, U.S. Geol. Survey Bull. 1094, pl. 4. 
thick. A 300-foot-thick formation consisting of impure spiculite and coquina overlics the McCarthy Shate in the McCarthy $\mathrm{C}-5$ quadrangle (Mackevett, 1971, 0. 15).

Several thousand feet of altered marine pyroclastics rocks of the Lower Jurassic Talkeetna Formation are present as lavas and tuffaceous sediments in the Talkeetna and Chugach Mountains (Crantz, 1965). Marine sedimentary rocks of Early Jurassic age occur in the upper Chitina valley. Thus the southeastern part of the Copper River basin may contain a Lower Jurassic rock facies transitional between the lava-bearing Talkeetna formation and the sedimentary rocks of the upper Chitina valley (Andreason and others, 1964, p. 138). Moffit (1938, p. 62) states that Middle and upper Jurassic rocks are widely distributed in the Chit ina valley, but occupy only a small total area. They include several thousand feet of tuff, shale, limestone, sandstone, and conglomerate. While the Jurassic sediments are considered to be of marine origin, one unit of tuffaceous conglomerate may be of interest with respect to possible uranium content. This unit, the Kotsina Conglomerate (Moffit, 1933, p. 62-64) unconformably overlies the Nikolai Greenstone and Triassic shale and limestone on the east side of the Copper River and north of the Chitina River between the Kotsina and Cheshnina valleys. The unit is at least 1,500 feet thick, and consists of pebbles of argillite, diorite, greenstone, and quartz in a tuffaceows matrix. Moffit $(1938, \mathrm{pl} .2)$ indicates the Kotsina Conglomerate underlies about 50 square miles and forms steep cliffs in the mountains.

The Middle Jurassic is represented in the sollthwestern part of the Copper River basin area by the Tuxedni Formation which overlies the Talkcetna Formation (Chapin, 1918, pl. 11; Crantz, 1960, 1965). It is considered to be entirely marine and to consist of buff sandstone, soft sandy shale, and a smaller anount of dark-brown arkosic sandstone containing "black minerals" (Chapin, 1918, p. 32). It may reach 1,000 feet in thickness in the Matanuska valley.

Creluceous marine sediments occupy a large part of the upper Chitina valley. The beds include black shale, sandstone, conglomerate, grit, and sandy shale, but no limestone. Large masses of granite and quartz diorite cut the beds. A distinct unconformity separates the Cretaceous from the older sediments and $i t$ is notably less folded and faulted. The cretaceous sediments in the chitina Valley and adjacent area have been described by Moffit (1938, p. 78) as forming a sequence with a total thickness of at lanst 6,000 feet, but nowhere is there a complete Cretaceous section.

More recently, Jones and Mackevett (1969) divided the Cretaceous rocks in the McCarthy quadrangle, north of the Chitina Valley, into five formations: the Kennicott, Moonshine, Schulze, Chititu, and McColl Ridge.

Following a general uplift at the close of the cretaceous or at the beginning of Tertiary time, a wide, low depression was developed in the Copper River region. Tertiary continental sediments, which included sandstone, conglomerate, siltstone, claystone, and locally, beds of coal, were deposited over this area. The rocks are considered to be Eocene on the basis of poorly preserved plant remains (Moffit, 1938, p. 97). Lava flows and pyroclastic deposits of Tertiary to Recent age are extensive around the volcanic centers in the Hrangell Mountains. 
Because glacial and alluvial deposits of nuaternary age now mask the older sediments in the basin by thicknesses of 600 feet or more in places and possibly as much as 3,000 feet (Andreason and others, 1964, p. 138), the thickness and distrihution of the Tertiary are not well known. A limited anount of information is provided by seven electric logs ror petroleum test wells, but detailed descriptions of the lithologies do not seen to be available. The combined thickness of the Quaternary and Tertiary sediments ranges from 0 to 2,630 feet, as shown by the top of the cretaceous. The thickest section was penetrated by Atlantic's Rainbow 1, 30 miles northwest of Gulkana Junction.

Tertiary sediments can be seen in outcrop in two general areas in the Copper River basin region: in the northeastern part between the Chistochina River and the Richardson llighway, and in the southwestern part north of the Glenn Highway and on the western side of the Talkeetna Kountains (Grantz, 1960a, 1960b, 1965).

Eocene sediments in the northeast part consist of more than 2,000 feet of section, which has been named the rakona Formation (Mendenhall, 1905, p. 52-53). The largest outcrop lies adjacent to the eastern side of Gakona Glacier and covers 20 lo 25 square miles. A basal conglomerate here is not less than 500 feet thick ind is made up of coarse, indurated igneous rocks that dip eastward and appear to pass beneath soft, fissile or massive, gray or buff-colored shales which, with inlerbedded gravel, sand, and lignite beds, extend to the head of the west fork of the Chistochina River.

I gneous Rocks

A wide variety of intrusive and extrusive rocks are present in the hills and mountains surrounding the Copper River basin. These were emplaced during numerous periods of activily. Flow rocks are exposed over large areas, but intrusive rocks, while widely distributed, crop out in relatively few places.

Volcanic flows are present as a major part of the Carboniferous and older sequences extending across the northern margin of the Copper River basin in the foothills of the Alaska Range. Lavas are also consticuents of the Carboniferous sequences in the Chitina valley (Andreason and others, 1964, pl. 24; and Chapin, 1918, pl. 11). A 4,000-foot sequence of basaltic lavas is present in the Wrangell Mountains as a part of the Permian Station Creek Formation (Smith and Mackevett, 1970, p. Q6).

The Talkeetna Mountains include a large core of quartz diorite and granite to the west of the Copper River basin. The intrusives range in age from Carboniferous to cretaceous. Tuffs and flows, in general andesitic but ranging in composition from rhyolite to basait, are also present in the Talkeetna and Chugach llountains as the lower part of the Talkeetna Formation. In this general area numerous small light-colored granitic and porphyritic intrusives are scattered hetween the Copper River and Klutina River. A belt of felsic rocks trending northeast between the headwaters of the Kashwitna River and the west fork of Kings River has been mapped by members of the Alaska State Survey (ADGGS, 1974, $p .14-15$ ). This bet has been mapped for 8 miles, but its total extent is not known. Rocks range in composition from quart? monzonite to granite, and lacally may be classified as 
alaskite. These rocks may have a potential for vein-type uraniun deposits.

Younger intrusives. Upper Jurassic to post-Eocene, occur in the Chitina valley and adjacent areas. These include granodiorite, quartz latite, quartz diorite, granite, and syenite (Moffit, 1938, p. 106-107).

The Triassic Nikolai Greenstone overlies the Lower Permian lavas and sediments and occupies numerous areas in the Wrangell Mountains and along on the north side of the chitina valley for $100 \mathrm{miles}$. It is a thick sequence of basalitic flows, well known because of the associated copper deposits in the Kennicott mines near McCarthy. The primary minerals in the lava, in order of abundance, are augite, labradorite, iron ores, apatite, olivine, and orthorhombic pryoxine (Moffit, 1938, p. 39). The lavas that actually make up the bulk of the Wrangell Mountains are those of the Vrangell Lava Formation of Focene and Quaternary age. Andesite is the chief rock type, but some basalt and dacite are present. Basaltic lavas and minor felsic extrusives and pyroclastics, containing Eocene plants, possibly equivalent to the Wrangell lava, are widespread in the southern Talkeetna Mountains.

The literature on the igneous rocks of the Copper River basin region indicates that basic compositions, especially basaltic and andesitic, may predominate. However, intermediate to acidic intrusives are present in a large part of the Talkeetna Mountains, and small amounts crop out in the southern part of the basin west of the Copper River and in the Chitina valley. Many types of botls sedimentary and igneous rocks were probably being eroded during deposition of the Eocene nonmarine sediments in the Copper River basin, and it appears that intermediate to acidic types could have contributed significani amounts.

\section{Structure}

The Lower Jurassic Seldovia and Talkeetna geanticlines trend through the northern part of the Chugach Mountains and the northern half of the Copper River hasin, respectively (Payne, 1955). Between the geanticlines lie sedimentary rocks deposited in the Matanuska geosyncline during the Mesozoic era. These sediments trend into the Matanuska Valley to the west and into the Chltina valley to the southeast. The Mesozoic sediments form a sequence having a thickness of at least 8,900 feet where the axis of the narrow Matanuska geosyncline crosses the southern part of the Copper River basin.

The Castle Mountain thrust fault, which is a major feature on the north side of the Matanuska Valley, probably extends eastward into the Copper River basin as a set of northeast-trending branch faults. Folding of the bedded rocks in the region is pronounced in the Carboniferous rocks but becomes progressively less so in the younger strata. Mesozoic beds on the western edge of the basin next to the Talkeetna Mountains are generally flat lying, except where faulted (Grantz, 1960). The Tertiary sediments are apparently mearly horizontal wherever found in the basin.

\section{Economic Geology}

It is estimated that about 3,500 square miles of the copper River basin is underlain by Late Mesozoic marine rocks that correlate with the sequence containing indications of petroleum in the cook Inlet. Sandstones of Middle 
Jurassic to Late Cretaceous age aro believed to offer the best possibilities for petroleum in the Copper River basin (USGS, 1964, p. 53). Evidence of petroleum consists of a fetid odor in a few of the Cretaceous beds, gas shows in test wells, and several gas seepages at the surface. As of 1969, eight unsuccessful wells had been drilled to depths ranging from 2,793 to 8,837 feet.

The Tertiary sediments are not considered likely to be petroleum reservoirs, but they may thicken locally and contain some oil of Mesozoic origin and methane gas. This possibility is of incerest because petroleum hydrocarbons are believed to sometimes provide a reducing environment for uranium deposits (Taucher, 1971, p. 5).

Humerous publications include cescriptions of the many ore deposits in the region, which, if the Mccarthy area is included, is one of the richest mineralized pares of Alaska. Fold and copper have been most developed. Excerpts from Berg and Cobb (1967, p. 37-65) are given in an effort to summarize the information on the geology and show the variety of minerals in the three recognized mining districts within the scudy orea.

\section{Chistochind vistrict}

The Mcsozoid rocks are coonomically important in the Chistochind district because they include the Nikolal Greenstone and overlying Chitjstone limestone, which, in the Kotsina-kuskuland area, are host to numerous copper lodes.

Copper, gold, silver, and molybdenum lodes in the Chistochina djstrict were prospected from about 1898 to 3940; the greatest exploration activity was prior to 1930. Iron, lead, zinc, and bismuth also occur in some of the lodes, but no attempt has been made to exploit these metals. Most of the prospects are between the Chitina River and the crest of tho Wrangell Mountains in an area drained by the Kotsina and Kuskulana Rivers. A rypicill copper deposit in the Nikolai Greenstone in the Coppes. Creek area is in sheared or brecciated greenstone and consists of small irregular veinlets of quartz, calcjet, and epidote, and subordinate bonnite, chalcopyrite, chalcocite, enargite, malachite, azurite, pyrite, and limonite. It commonly is within a few hundred Feet of the top of the groenstone.

The most thoroughly explored copper deposit on Copper Creek is the Mullen prospect where the lode consists of veins of calcite, pyrite, boxnite, chalcopurite, coveluite, limonite, malachite, and azurite in brecciated limestone. Samples of the two principal veins assayed 1.55-5.82 percent copper, a trace of gold, and as much as 0.28 ounces of silver per ton. Total indicated resources of the two veins is abont 1,360 t:ons.

Other minerals reportedly present in the Chistochina district include tetrahedrite and bismuthinite on the Kluvesna River; sphalerite and galena with copper and iron minerals have been found in the slana areas in the northeastcrn part of the Copper River basin; molybdenum, mainiy in a pegmatits dike. was found on Rock Creek, also in the Slana area. 
Nelchina District

The Nelchina district is the area drained by east-flowing tributaries of the Copper River from Gulkana on the north to, but excluding, the Tusnana River on the south.

Lodes in the Nelchina district have been prospected for gold, silver, manganese, and chromite. The gold lodes, which also contain a little copper, lead, and zinc, are mainly in an area southeast of Tonsina Lake, the site of considerable exploration early in the present century. On Dust Mountain, at the northeastern end of the intrusive, a body of massive chromite as much as 10 feet thick is exposed for a distance of 75 teet. Samples of the richest material assay 36.0-57.7 percent chromite, with a chrome-iron ratio of 1.20-3.06. Assays also show traces of nickel and platinum.

\section{Nizina District}

The district is dominated in the south by the Chugach Mountains, in the northeast by the St. Elias Mountains, and in the northwest by the wrangell Mountains. Within these regions of high relief and large fields of perennial ice and snow, the only large lowland area is the valjey of the Chitina River.

Lodes containing coppex, silver, gold, lead, zinc, molybdenum, and nickel occur in the Nizina district, but only the lodes bearing copper and precious metals have been the sources of substantial amounts of ore. The period of greatest lode prospecting and mine development was from 1900 to 1938 , when more than a billion pounds of copper was recovered, nearly all from the Kennicott mines. The group of properties known as the Kennecott mines includes the Bonanza, Jumbo, Erie, and Mother lode mines, Incated at altitudes of 4,000-6,000 feet in the mountains about 7 miles north and noxth-northeast of McCarthy. The underground workings in the four mines were interconnected and totaled about 70 miles in length, the deepest workings reaching an altitude of about 2,800 feet. The mines exploited several ore bodies, the most important of which were the famous Bonania and Jumbo veins--veins that were unique in that they constituted the largest masses of almost pure copper ore that have ever been discovered. The Kennecott mines were in almost continuous operation from 1911 to 1938, dirring which time they yielded most of the copper produced from Alaska. The Kennecott ore bodies were in Chitistone limestone a little above the contact with the Nikolai Greenstone. The ore occurred as veins, irregular massive replacements, and stockworks, mostly in partiy dolomitic beds in the lowest 300 feet of the Chitistone Limestone. About three-fourths of the ore mined at kennecott consisted of sulfide minerals, of which an estimated 95 percent was chalcocite. The remaining sulfides were chiefly covellite and sparse to trace amounts of enargite, bornite, chalcopyrite, luzonite, tennantite, pyrite, sphalerite, and galena. Besides the important copper deposits, other lodes in the Nizina district have been explored for gold, silver, lead, molybdenum, antimony, and nickel.

\section{Previous Radioactivity Investigations}

The USGS examined the southwestern part of the Wrangell Mountains on the north side of the upper Chitina valley near McCarthy (Moxham and Nelson, 
1952, p. 1-3). Apparently littlc or no data on the mines and ores in the district are available. Results of tests on nine samples collected in the McCarthy area by Moxham and Nelson are given helow:

\begin{tabular}{|c|c|c|c|}
\hline Sample & Location & Type of material & $\begin{array}{l}\text { Radioactivity } \\
\text { (in percent eU) }\end{array}$ \\
\hline 10 & Dan Creek & $\begin{array}{l}\text { Shale (Kennicott } \\
\text { formation) }\end{array}$ & 0.002 \\
\hline 11 & Dan Creek tributary & Panned concentrate & 0.000 \\
\hline 12 & Rex Creek & $\begin{array}{l}\text { Shale (Kennicott } \\
\text { formation) }\end{array}$ & 0.002 \\
\hline 13 & Young Creek & Panned concentrate & 0.001 \\
\hline 14 & North of McCarthy & $\begin{array}{l}\text { Shale (McCarthy } \\
\text { formation) }\end{array}$ & 0.000 \\
\hline 15 & North of McCarthy & $\begin{array}{l}\text { Greenstone (Nikolai } \\
\text { Formation) }\end{array}$ & 0.002 \\
\hline 16 & Near Kennicott & Granite & 0.001 \\
\hline 25 & $\begin{array}{l}\text { O'Neill Mine, Dan } \\
\text { Creek }\end{array}$ & Sluice-box concentrate & 0.000 \\
\hline 86 & $\begin{array}{l}\text { Chititu ilines, Inc. } \\
\text { Rex Creek }\end{array}$ & $--_{-}-\mathrm{do}-\mathrm{C}_{-}-{ }_{-}$ & 0.000 \\
\hline $\begin{array}{l}\text { Spots } \\
\text { checks }\end{array}$ & (1) & $\begin{array}{l}\text { Shale (Kennicott } \\
\text { formation }\end{array}$ & Insignificant \\
\hline
\end{tabular}

Teveral localities.

A single sample of the heavy-mineral fraction of gravel from Golconda Creek, a tributary to Chikina and Chitina Rivers, produced el of 0.004 percent.

The area in the Chugach Mountains including the west tributaries to the Copper River between the Tiekel and Gulkana Rivers has been called the Klutina district. Twenty-seven radinactivity measurements of rocks and stream concentrates from the area, taken from Moxham and Nelson (1952, p. 4), ranged between 0.001 and 0.003 percent el.

Several localities in the extreme northeastern part of the Copper River basin, in the chistochina district, were tested during 1953 and 1954 :

\begin{tabular}{|c|c|c|c|}
\hline Locality & Type of deposit & Radioactivity & References \\
\hline Rock Creek & $\begin{array}{l}\text { Molybdenite-bear- } \\
\text { ing permatite }\end{array}$ & $\begin{array}{l}0.004 \text { percent el } \\
\text { or less }\end{array}$ & $\begin{array}{l}\text { Hedow and others, } 1953, \\
\text { p. 6, 7; Nelson and } \\
\text { others, } 1954\end{array}$ \\
\hline $\begin{array}{l}\text { Mineral } \\
\text { Point area }\end{array}$ & $\begin{array}{l}\text { Altered shear } \\
\text { zone containing } \\
\text { copper, gold. } \\
\text { silver, and traces } \\
\text { of nickel(?) }\end{array}$ & $\begin{array}{l}0.001 \text { percent } \\
\text { eU }\end{array}$ & $\begin{array}{l}\text { Wedow and others, } 1953 \text {, } \\
\text { p. 6, } 8 ; \text { Nelson and } \\
\text { others, 1954 }\end{array}$ \\
\hline $\begin{array}{l}\text { Glenn High- } \\
\text { way between } \\
\text { Slana and } \\
\text { Mineral Lake }\end{array}$ & $\begin{array}{l}\text { Tests of con- } \\
\text { centrates from } \\
\text { gravels of } \\
\text { streams cross- } \\
\text { ing highway }\end{array}$ & $\begin{array}{l}0.003 \text { percent } \\
\text { eU or less }\end{array}$ & $\begin{array}{l}\text { Wedow, Killeen, and } \\
\text { others, 1954, p. 16-18 }\end{array}$ \\
\hline
\end{tabular}




\begin{tabular}{|c|c|c|c|}
\hline Locality & Type of deposit & Radioactivity & References \\
\hline $\begin{array}{l}\text { Silver Creek } \\
\text { area }\end{array}$ & $\begin{array}{l}\text { Quartz veins, con- } \\
\text { taining silver- } \\
\text { bearing galena } \\
\text { and tetrahedrite } \\
\text { with some gold } \\
\text { cutting diorite }\end{array}$ & $\begin{array}{l}\text { Veins contain } \\
0.001 \text { percent eu; } \\
\text { diorite contains } \\
\text { as much as } 0.005 \\
\text { percent eU }\end{array}$ & $\begin{array}{l}\text { Wedow, Killeen, and oth } \\
\text { 1954, p. 16-18; Wedow a } \\
\text { others 1953, p. 6, 8; it } \\
\text { and others, } 1954\end{array}$ \\
\hline $\begin{array}{l}\text { Indian } \\
\text { group }\end{array}$ & $\begin{array}{l}\text { Quartz veins } \\
\text { containing } \\
\text { silver-bearing } \\
\text { galena and } \\
\text { tetrahedrite, } \\
\text { chalcopyrite, } \\
\text { malachite, and } \\
\text { azurite }\end{array}$ & $\begin{array}{l}0.004 \text { percent } \\
\mathrm{eU}\end{array}$ & $\begin{array}{l}\text { Wedow and others, } 1953 \text {, } \\
\text { p. 6, 7; Nelson and } \\
\text { others, } 1954\end{array}$ \\
\hline
\end{tabular}

An anomalous radioactivity reading was reported from an a irborne survey. R.G. Bates recorded in Trace Elements Preliminary Reconnaissance report A-1726, December 3, 1953, a scintillometer reading of $1.0 \mathrm{mr} / \mathrm{hr}$ in the canyon of the Chitistone River. The background was $0.15 \mathrm{mr} / \mathrm{hr}$. No ground investigation was made. Although only a single reading was reported, it seems to be significantly high and to warrant follow-up testing. The mouth of the Chitistone River valley is 10 miles east of McCarthy.

\section{TERTIARY DEPOSITS AND GRAIHITIC ROCKS \\ OF THE EAGLE-CHARLEY RIVER AREA}

A belt of nonmarine sedimentary rocks of Upper Cretaceous to Pliocene(?) age lies along the south side of the Yukon River fron the International boundary to Woodchopper and Webber Creeks, which are east of Circle (fig. 17). The Upper cretaceous-Tertiary sediments lie within a few miles of (and are topographically lower than) the large Mesozoic, granitic Charley River batholith to the south, which presents a favorable situation for the accumulation of sedimentary-type uranium. The sediments of the belt are within the 3:250,000 Charley River and Eagle quadrangles.

The geology of the Charley River quadrangle has been mapped by Brabb and Churkin (1969), and the geology of the Eagle quadrangle was compiled by Foster (1972). The Upper Cretoceous-Tertiary deposits of the Eagle-Circle area were specifically studied by Mertie (1942), who also published a repart on the general geology of the region (1930).

The Upper Cretaceous-Tertiary belt occupies a trough formed along the Tintina fault zone (or Tintina Trench), which is an extension of the Rocky Mountain Trench and a major structural feature in east-central Alaska. The fault zone is also referred to as the Eagle Trough. The area underlain by Upper Cretaceous-Tertiary sediments along the trough is from 2 to $15 \mathrm{miles}$ wide and $80 \mathrm{miles}$ long. The area is one of low, rounded ridges, and con$t$ inues northwestward along the Yukon River valley into the loess-covered terraces and lake-dotted plain of the southeastern part of the Yukon Flats. 
The hills are heavily wooded. South of the trough area, mountains of the Yukon-Tanana Upland rise gradually to altitudes of over 5,000 feet. On the north side of the trough and the Vukon River, the Ogilvie Mountains form a rather rugged group with a maximum altisude of about 4,600 feet.

The Yukon-Tanana Upland is drained by north-flowing streams that rise in the highland to the south and have superimposed courses to the Yukon River in narrow valleys. The major cributaries to the Yukon River on the north side of the trough are the Mation and Kandik Rivers.

The town of Eagle, on the Yukon, is near the southeastern end of the trough and is accessible by the Taylor Highway. The northwestern end of the trough is near Woadchopper Creek, which is a southern tributary of the Yukon. A short road connects the lower parts of Woodchopper and Coal Creeks. The town of Circle is located a little farther downstream on the Yukon and is accessible by the Steese Highway. Other parts of the area can be reached by boat on the Yukon River and its tributaries. Short landing fields are located at Eagle and Circle, and at one time landing strips were used at the Nation and Woodchopper settlements.

The climate is similar to that of the fairbanks area. The yearly mean ansual temperature is $25^{\circ} \mathrm{F}$, and the average precipitation is 10 inches. Permafrost is discontinuous throughout the area.

The Tertiary and Cretaceous sediments in the trough are in a synclinal belt and they probably are in sedimentary contact with both the metamorphic and granitic rocks of the Yukon-Tanana Upland on the south and the highly indurated Precambrian, Paleozoic, and Mesozoic sediments of the Ogilvie Mountains on the north. While the Tertiary belt closely follows the course of the Yukon River, the contacts are separated by a narrow belt belonging to the Precambrian-Tertiary sequence of the Ogilvie Mountains or the Kandik Basin.

\section{Sedimentary Rocks}

A large number of geological formations and groups ranging in age from Precambrian through Tertiary have been mapped in the area (Brabb and Churkin, 1969; Foster, 1972). The north side of the trough and Tintina Trench is occupied by one of the most complete stratigraphic sections in Alaska. The area north of the Yukon River is termed the Kandik Basin. The pre-Tertiary rocks south of the rintina fault do not correlate with those on the north slde because of the great right-lateral displacement along the Tintina fault, which may be as great as 260 miles (Davies, 1972). The rocks on the south side of the trough are mostly metamorphosed sediments and greenstones of Paleozoic age and Mesozoic granitic rocks. Descriptions of most older rocks do not seem to suggest that they would be favorable hosts for uranium. However, one older sedimentary formation that may possibly be of interest to uranium explorationists is the Nation River Formation, which is exposed on both sides of the Yukon River from a short distance downstream from Eagle to about 6 miles below the mouth of the Nation River. Early reports dated the Nation River Formation as probably Permian, but Brabb and Churkin (1967) have shown that it is most likely Late Devonian. The Nation River Formation consists of up to 4,000 feet of nonmarine clay shale, sandstone, and conglomerate which has been said to resemble Tertiary sediments. 
Study of the Upper Cretaceous-Tertiary sedimonts in the Eagle-circle district by Mertie (1942) was primarily for determining the source of the placer gold which was mined from streams south of the Yukon River, most notably at American, Coal, Woodchopper, and Fnurth of July Creeks.

Mertie's conclusion was that the immediate source of the gold was the Upper Cretaccous-Tertiary sandstones and conglomerates but the original source was the granitic rocks to the south. The Upper Cretaceous-Tertiary sediments were probably laid down in an alluvial basin which was originaliy much more extensive than it is today.

Outcrops of the Upper Cretaceous-Tertiary rocks are not extensive because they are generally little indurated and nonresistant to weathering, though some units are locally well indurated. Exposures can be found in stream valleys. However, no complete section is exposed anywhere and partial sections have not been combined to yield a composite section.

Generalized descriptions of the lithologies are taken from Mertie $(1942$, p. 224-225):

The materials composing the rertiary conglomeratic rocks and sandstones tond to be fairly uniform in iithologic character, but it some lexilities where these rocks lie close to the underlying Paleozoic and Mesozoic rocks their composition varies markedly from the usual composition. At most places the pehbles of the conglomoratic rocks consjst of chert of various colors, quartzite, and vein guart', which are the types of rocks that are practically indestructible, except by abrasion. I,oc-3l]! and especjrlly near the base of the formation, pebbles and cobbles of granitic rocks, greenstone, schist, argijlike, and cvon limestone have been observed. In most of the conglomeratic rocks, the pebbles are relatively small, the maximum diamcter being 4 or 5 inches and the average between 1 and 2 inches. But at certain horizons near the base of the sequence, coarse conglomerate has been observed, as Eor example on American and crooked crecks. The best example of coarse conglomerate, howover is along the ridge that separates Crooked Cxcek Erom Trout crook. Here large residuil cobbles and boulders of quartzite as much as 2 feet or more in djametex lie at the surface and represent the dcbris derived from the surficjal alteration or slaking of coarsc conglomerate. The sandstones of the series aro not materially different from the conglomerates, except for the smaller size of the component grains. Under the microscope the finer rock forming mintrals, loth of the conglomerates and sandstones, are found to be quartz, orthoclase, plagioclase, hornblende, and mica, together with iron ores, zircon, garnet, and other heavy minerals derjued from granitic rocks and crystalline schists. At places the heavy minerals are concentrated in stratjfjed layers and in creneral appear to be more plentiful than in thejr original parent rocks. Most of the shale is sandy, but in the stratigraphic horizons where coal is found clay shales are also found. The coal that occurs in these tertiary rocks was studied years ago by colliex and was found from numerous analyses to be dominantly a high-grade lignite, with some subbituminous varieties. At the present time, with ample wood for domestic use and with cheap oil for large-scale mining opexations, these low-grade coals are of littie ecomomic importance. 
The base of an Upper Cretaceous-Tertiary conglomerate along the west bank of Mission Creek appears to lie directly on pre-Upper Cretaceous granite, and in other areas arkosic sandstone can be observed to have been locally derived directly from granite, with which it is in contact. Besides granite, other constituents of the conglomerate include chert, schist, quartz, quartzite, and greenstone. Fragmental plant remains are interbedded with sandstones at various localities. Gold was found to be irregularly distributed in certain sano'stone and conglomeratic beds and almost certainly was derived from weathering of the granitic rocks.

Igneous Rocks

Several Paleozoic and possible Precambrian igneous rock units have been mapped in the Eagle and Charley River quadrangles. A sequence of basalts and sedimentary rocks believed by Mertie to be Early Mississippian are known as the Circle Volcanics. Mesozoic granite, including some diorite and related rocks, form a large complex south of the Yukon River. Tertiary lava flows, mainly rhyolite but including some dacite, are present. This study is concerned principally with the Mesozoic granitic rocks which appear to have the greatest potential as uranium source rocks for the Upper cretaceous-Tertiary trough sediments and as possible hosts for vein-type uranium.

The Mesozoic granitic rocks occupy a zone $80 \mathrm{miles}$ long and from 10 to 50 miles wide with smaller outlying masses of similar rocks both north and south of the main massif. The total area is 1,900 square miles. The main body, the Charley River batholith, is named from the Charley River, which cuts the batholith about halfway between Eagle and Circle. Other batholiths and smaller granitic bodies occupy much of the Yukon-Tanana Upland in east-central Alaska.

The felsic rocks identified by Mertie (1930, p. 150) consist of muscovite granite, alaskite, muscovite-biotite granite, amphibolite, epidote granite, and quartz monzonite. Subsilicic types are granodiorite, quartz diorite, and diorite. Basic rocks include gabbro, peridotite, and pyroxenite. The granitic and dioritic rocks, however, are most typical of the region. Locally, primary granite gneiss is developed along the contact of the intrusives with the country rocks. Tertiary and Mesozoic dikes of both felsic and mafic compositions are common near the intrusive rocks. Ultramafic rocks of the Eagle quadrangle are described by Foster and Keith (1974).

The plutonic rocks of the main complex in the southwestern part of the Charley River quadrangle are termed adamellite (quartz monzonite) by Brabb and Churkin (1969). Their description follows:

Medium-to coarse-grained adamellite. Biotite is chief accessory mineral. Hormblende less abundant. Muscovite and gamet occur locally. Adamellite has zenoliths of schist and i.s cut by aplite dikes. The adamellite forms generally structureless bodies discordant with surrounding schist, but in some places the adamellite seems to grade into guartz biotite gneiss.

The Mesozoic plutonic rocks in the Eagle quadrangle are described by Foster (1972): 


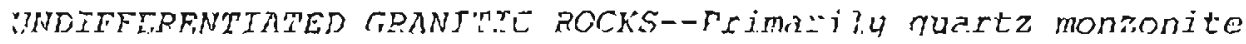
ane ramoristite, hut imeludos rranite to diorjte with local aplite, alaskits, anri pegmatite. Fire tio coarse grained: equigranular to

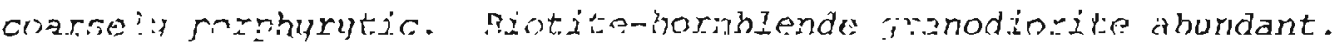
corimon, y crops out in tows. Most of Iargex plutons probably Mesozoic in age but includes mertiary plutions.

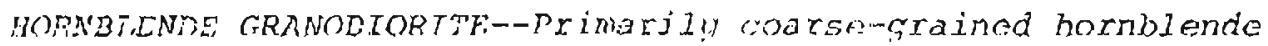
granndicite and fuartz monzonite but includes some gabbro and syenite. prohdb.l!" Meroznjc in age.

SYENTTE OF MOUNT VETA-Primarjiy hornblonde syenite porphyry, but locisly equigranular. Treindes hornblends quartz-monzonite and dioriks. Fotassium-argon age on hornblemen of $177 \mathrm{~m} \cdot \eta \cdot+5 \mathrm{~m} \cdot \mathrm{y}$. (Potassium-argon report no. 54 (Mon? Park), 3969, hy Joven Essen).

While both Mount Veta and Mount Fairplay are about 75 miles south of the Yukon River in the Yukon-Tanana Upland and outside the Eagle-Circle region, the syenitic rocks arc of interest hecause of the possibility of uranium associations (Foster, 1967). The Mounl Fairplay area has long been known to have a very high radicactivity background, and even though it was the sitc of a uranium stakina "rush," no commercial uranium deposits were found.

Another rock unit of possible interest because it may consist of Precambrian orthogneiss is the Pelly Gneiss (Mertie, 1937, p, 201-202). This unit occurs in scattered, rather ill-defined areas throughout much of the Yukon-Tanaria Upland of eastern Alaska.

\section{Structure}

The most significant structural feature of the Eagle-Circle district is the Tintina Trench, extending 600 miles or more from Canada into eastcentral Alaska. Right-lateral displacement has been calculaced to be as much as 260 miles. The trench forms a lowland now partly occupied by the Upper Cretaceous-Tertiary sediments of the Eagle-Circic district. Subordinate faults and folds related to movement in the fault zone are well developed in Precambrian-Paleozoic rocks and Crotacenus granite. Displacement of Quaternary alluvium shows that movement has continued (Davies, 1972).

The Tertiary rocks have been deformed into appressed folds. Bedding, though locally dipping northward, dips dominantly southward and it is presumed that the Tertiary rocks lie in a belt of appressed folds overturned to the north.

\section{Economic Geology}

Gold in placer workings have long been known in the region. All the streans mined for gold lie south of the Yukon River, as do the granitic intrusive bodies. The valleys of coal and Woodchopper creeks contained the most important placers in the Upper Cretaceous-Tertiary belt. While mining has been dormant for a number of years, a dredne of coal creek is currentiy being reactivated. 
The Upper Cretaceous-Tertiary deposits have acted as a proximate source for the gold, which in turn was concentrated in various tributaries of the Yukon River. The original source was the granitic plutons south of the Upper Cretaceous-Tertiary belt. All the important placers are on the valley floors, but less significant placers occur as bench deposits on higher and older erosional surfaces. Silver and platinum are alloyed with the placer gold: up to 18 percent silver and 0.42 percent plat inum.

Lode deposits in the immediate vicinity of the Eagle Trough are scarce. Mineralization was staked in 1948 on sulfide-bearing rock containing cobalt bloom and a basaltic greenstone on Eagle Bluff just outside the town of Eagle along Mission Creek (Saunders, 1952). Very little work has been done on the prospects. A few lode prospects in the circle district were explored for gold, copper, and lead. A copper prospect 50 miles east of Eagle on Copper Creek about 6 miles above its confluence with the Charley River occurs in highly metamorphosed rocks which may be a roof pendant in the Charley River batholith. Chalcopyrite, malachite, and azurite are the chief metallic minerals (1/hite and rolbert, 1954, p. 7-9).

Some lowagrade coal has been exposed at several stream valleys that cut the Upper Cretaceous-Tertiary beds, but apparently it is not suitable for exploitation. One site, believed to be in the Nation River Formation, has been mined for coal, and about 2,000 tons were used to fuel river streamers (U.S. Geol. Survey, 1364, p. 80). 0il shales, containing as much as 28 gallons of oil per ton, within the Triassic-Lower Cretaceous Glenn shale, are well known but relatively thin (Mertie, 1930, p. 132). An occurrence of asbestos has been found near the center of the Eagle quadrangle in a serpentinized mass that appears to have intruded metamorphic rocks (Foster, 1969).

Radioactivity Investigations

Several reconnaissance investigations for radioactivity by the U.S. Geological survey and one by DGGS has been conducted in west-central Alaska. Wedow (1954) investigated the Eagle area in 1948 on behalf of the U.S. Atomic Energy Commission, and reported the following data:

Reconnajssance of radioactive deposits in sedimentary rocks of Proterozojc and Paleozoic age, and granite of Mesozoic(?) age togethex with its Tertiary sedimentary derivatives, was conducted in the Eagle-Nation area, east-central Alaska in 1948. None of the rocks examined contains more than 0.003 percent equivalent uranium except for black shale beds in the upper Mississippian Calico Bluff formation and in granite of Mesozoic(?) age and its sedimentary derivatives. The more radioactive black shale beds in the Calico Bluff formation range in thickness from $1 / 2$ to 7 feet. Two units neat the base of the formation appear to be persistent in the area: Radioactive unit $A$, with an average thickness of 6.6 feet, contains an average of 0.007 percent equivalent uxanium and 0.004 percent uramium; radioactive unit $B$, with an average thickncss of 5.2 feet, contains an average of 0.006 percent equivalent uranjum and 0.003 percent uranium. Phosphatic pellets from unit $B$ at one locality contain 0.022 percent equivalent uranium, 0.019 percent uranium, and 15 percent $\mathrm{P}_{2} \mathrm{O}_{5}$. Samples of granite of Mesozoic(?) age and its Tertiary sedi- 


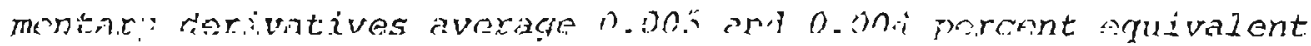

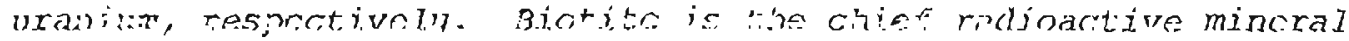
in the ryante ond its radinartivitu is ascribad to tho presence

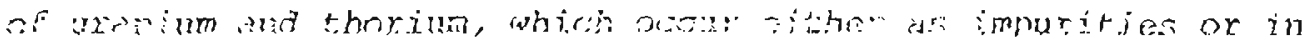
minuth in ilusinas of othor, as lut unidentifind, minerais. Traces

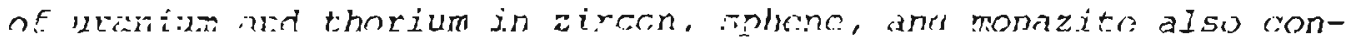

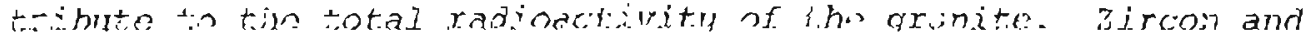

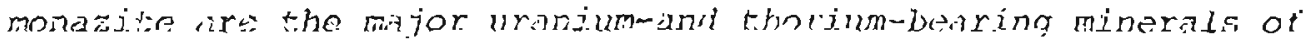

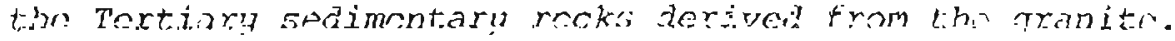

The investigators concluded that the radinactivity at the Copper creek prospect was dur to uranium as impulities in horrite and malochite. They also noted that tho investigations wers l imiten and that greater concentrations may nccur in the area of the mineralized roof pendant in the batholith.

Results frnm other areas tested by orrs in west-central Alaska (Eakins, 1969, p. 13-14) are given:

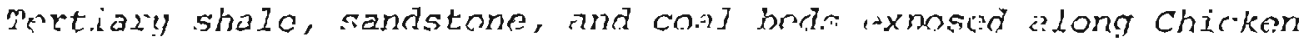
croek at the town of Chicken and in a rivel pit noar chicken did

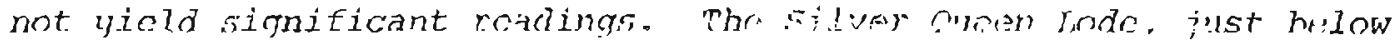
the hiqhwau about four miles north ot Chick'n and near Miles Esst 71 did not snow measureable radionctivith. The nrospect consists of a in-font tunnel following a golvat zone with showings of aslend.

Twn feet of gouge in a fialt zrno $j$ il a sonspicunus outcrop of marhle in a road cut at wite post 7 lit grat three tjmes tho borkgrourd count, ox between 0.0 .3 and $0.04 \mathrm{Mr} / 1 / \mathrm{r}$. Tortiary sandstones and shales exposed in horrow pits along tho Taylor Highway from $\rightarrow$ fow milus south of Eagle to Eagle contain sandstone shales, and siltstones. The vere fine-grained silty sandstones and siltstones wer: noticrahly higher in radionctivitu than the clcancr, corrses sandstones. Counts up to $0.03 \mathrm{Mr} / \mathrm{Hr}$ were obtained.

A Eoot traverse along American Creek From Eagle south for five miles was made to examine Tertiurul sandstonos and conglomeratos cxposed in blutfs along the creek. At three locations localized anomalies were encountored where faults cut thmse beds. The maximum readings wre $0.03 \mathrm{Mr} / \mathrm{Hr}$. Mission Croek enters the Yukon just west of the town of Eagle near the base of Earls Bluff. The prominent Fagle Bluff stands between Mission Creek and the Yukon. Tn tho l940's several claims covering showings of gold, copper, nickel, and cohalt were staked along a fault zone on the Mission Creok side nf Farle [3luff. A foot traverse in this area ilid not produce any radioactive anornalies, but all seven clajms wero not examined in detail. No mining has been done on the ciaims.

Hrequent checks with countars along the Yukon River between Eayle and the Canadian borler revealed nu anomalies in the raleozoic rncks exposed. The Nation River corglomerete exposnd no anomalons rendings. The Mississipnian Calico Bluff formation exposed on Calicn BluEf about eight miles downriver from fiagle has heen roported to contail: radinactive hlack shales. The writer measired readings up to 0.05 Mr/Hr in black shales near the bise of the bluff. A rlimb from the river to thin top of thes hinff produced lesser raadings. Tertiarly beds exposed on the south side of the yuton from two to saven miles 
west of the mouth of the Seventymile Rivar produced only very low radioactivity. A maximum reading of $0.05 \mathrm{Mr} / \mathrm{Hr}$ was obtained from one narrow brecciated zone cutting the beds.

The Upper Cretaceous-Tertiary nonmarine sediments in the Eagle Trough are essentlally untested for uranium. The beds extend 80 miles along the northern boundary of a region containing extensive granitic plutons which have displayed radioactivity anomalies from the air and on the ground. For the most part, the sandstones contain 0.002 percent eU. At least part of the Upper Cretaceous-Tertiary sequence has been shown to have been derived from the granitic bodies and to contain carbonaceous and coaly material. However, beds are generally folded and dips frequently are between $45^{\circ}$ and $90^{\circ}$.

Many of the alkalic plutons in a broad region of the Yukon Tanana Upiand in east-central Alaska offer possibilities for nonsedimentary uranium deposits. Scattered small areas have been investigated and el contents of up to 0.007 percent and averaging 0.005 percent have been found in the granite and related rocks.

\section{TERTIARY COAL-BEARING BASINS, NORTH FLANK OF THE ALASKA RANGE}

Nonmarine coal-bearing sediments occur intermittently in a 250-milelong belt on the north Flank of the Alaska Range from a point 25 miles east of Farewell eastward to $20 \mathrm{miles}$ east of Donnelly on the Richardson Highway (see Geologic Map of Alaska: Dutro and Payne; 1957). These sediments lie mostly in the northern foothilis of the Alaska Range physiographic division of Wahrhaftig (1965, p. $35-36$, pl. 1). The foothills consist of flat-rcpped, east-trending ridges 2,000 to 4,500 feet in altitude which form a bel: reaching 20 miles in width along the Nenana River. Most of the contailis are unglaciated, but some valleys were widened during the Pleistocene Epoch by glaciers from the Alaska Range. Streams draining the north slope of the Alaska Range flow northward through the foot hills to the Tanana River valley, except in the western part of the belt, where drainage is into the headwaters of the Kuskokwim River. The Alaska Range forms a great arc extending 600 miles from the Canadian border to Lake Clark in southwestern Alaska, where it merges with the Aleutian Range. The crest of most of the range is between 7,000 and 9,000 feet in altitude, but mountain masses are higher.

Most of the Tertiary basins in the foothills are relatively accessible; none are too remote from towns, and the terrain is not extremely rugged. The belt is crossed by two north-south highways and a railroad: the Richardson Highway crosses the belt near the eastern end, and the Anchorage-Fairbanks Highway and the Alaska Railroad cross the Alaska Range through the Nenana River valley at the eastern edge of Mount McKinley Park. Precipitation is less than 20 inches a year. Permafrost is extensive. The foothills belt is covered by the Big Delta, Fairbanks, Kantishna River, Mount Hayes, Healy, and Mount Mckinley 1:250,000 topographic maps. Geologic maps of eight 15-minute quadrangles were recently issued and provide detailed information on much of the Healy and Fairbanks quadrangles (Wahrhaftig, $(970 a-h)$. The surficial geology of the central part of the Alaska Range and Nenana River valley is covered by Wahrhaftig (1958). 
The best known part of the Tertiary coal-hearing belt is along Healy Creek, where Usibelli Coal Mines, Inc. produces coal near the settlement of Heaiv and Sisntrana. The coal is uscd to fuel two power plants, which sorve Fairbanks and the military bases in the region. Healy creek is part of the Nenana coal field, which includes several separate but closely grouped basins in a 40-mile-long area, mostly between the Nenana and Wood Rivers. This group of basins includes Holy Creek, Lignite Creek, Rex Creek, Tatlanika Creek, Mystic Creek, and Wood River coal bains. The Nenana coal field has been described by Martin (1919). Farnes and others (1951), and Warhiaftig and others (1969).

Another locality where coal has been mined from the Tertiary beds on the north flank of the Alaska Range is the Jarvis coal field, located 100 miles east of Healy on the east side of the Richardson Highway near Donnelly.

Little detailed Information is availabie on other Tertiary basins in the belt, but it is hoped that the following discussion, which mostly describes the rocks in the Nenana coal Field on the north flank of the central part of the Alaska Range, will apply somewhat to all the Tertiary coal tasins in the belt.

\section{Sedimentary Rocks}

Rocks in the valley of Healy and Lignite Creeks include the Precambrian (or Paleozoic Birch Creek Schist), the Tertiary Coal-Bearing Group, and the Tertiary Nenana gravels. For lack of a more up-to-date stratigraphic table, the one below (table E2) is reproduced from Barnes and others (1951) with the understanding thal the Birch Creek Schist may be wholly or partly Paleozoic in age and that the Coal-Bearing Group has been revised upward from a formation to group status and formally divided into five new formations (Wahrhaftig and others, 1969).

Table F.2. Generalized stratigraphy of the Nenana coal field,

\begin{tabular}{|c|c|c|c|}
\hline Apo & Formalion & Description & $\begin{array}{l}\text { Thlckmpss } \\
\text { (feut) }\end{array}$ \\
\hline Quinceruary & $\begin{array}{l}\text { Termur ampels } \\
\text { Cucol)(ormity- }\end{array}$ & & $0-200$ \\
\hline \multirow{8}{*}{ Tortlary } & \multirow{5}{*}{ Senana gravol } & 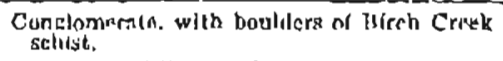 & $210+$ \\
\hline & & 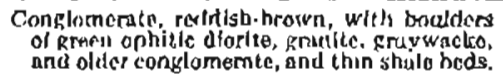 & \multirow{2}{*}{2,100} \\
\hline & & 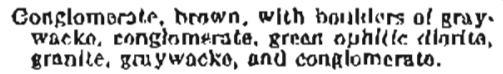 & \\
\hline & & 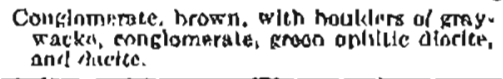 & 1,000 \\
\hline & & $\begin{array}{l}\text { Conplomemite hropes, with holilders of gray. } \\
\text { ryartie, conglonisroto, and dacisn. }\end{array}$ & 000 \\
\hline & & 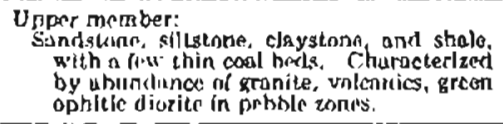 & $\begin{array}{l}50 M- \\
945\end{array}$ \\
\hline & $\begin{array}{l}\text { Coal-bearings } \\
\text { formition }\end{array}$ & 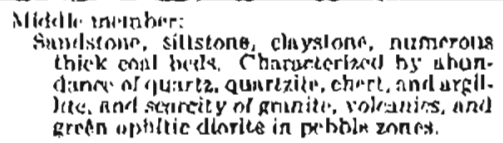 & 1,000 \\
\hline & & 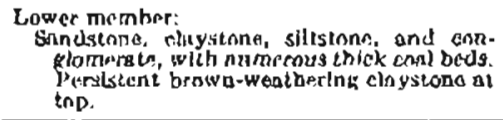 & 1,800 \\
\hline Pr.Cumbrín & $\begin{array}{l}\text { Birch Cremt } \\
\text { schise }\end{array}$ & Quntur-mica scinist. & $i$ \\
\hline
\end{tabular}


A discussion of the many Paleozoic and Mesozoic formations and intrusive rocks composing the Alaska Range is beyond the scope of this study, which is concerned mostly with the Tertiary Coal-Bearing froup. The underlying Birch Creek Schist, however, is prominent in the Tertiary basins and deserves mention. It consists of highly contorted quartz-mica and quartzchlorite schist with some interbedded phyllite, argillite, and black carbonaceous schist. These are all cut by numerous veins of milky quartz. When fresh, the schist is green. It weathers to shades of gray, green, and black, and becomes fissile.

Another unit that is exposed in the hills north of Healy is the Totatlanika Schist of pre-Devonian age. It consists of gray to black quartz muscovite and graphitic schist with interbedded argillite and metamorphosed volcanic rocks.

The Tertiary coal-bearing sequence lies unconformably on the Birch Creek schist in the Nenana coal field. The coal-bearing rocks are well exposed in the Healy Creek valley and in the open-pit coal mines operated by Usibelli. Figures E6 and E7 show the structure of the coal-bearing beds in the Healy Creek valley and the relation to adjacent formations. Figure Ef shows the structure of Lignite Creek. The geology has been mapped on the Healy D-7 quadrangle (Wahrhaftig, 1970C).

The Coal-Bearing Group is up to 2,000 feet thick and shows a wide variation in thickness and lithology within short distances. Apparently it was deposited in valleys or depressions in the Birch Creek Schist. Later deformation tilted the beds and in places formed synclines. It is unconformably overlain by up to 4,000 feet of the Tertiary Nenana Gravel. South of the Healy Creek valley the beds are fairly flat lying for a distance of several miles.

\section{Structure}

The Birch Creek Schist basement is intensely deformed, tightly folded, and crumpled. In many places the bedding has been obliterated. The foliation strikes east and northeast and dips to the south. The Totatlanika Schist is generally less deformed than the Birch Creek Schist.

The uranium potential of the Tertiary Coal-8earing Group is of principal concern here, and a discussion of the structure of this sequence is reproduced from Barnes and others (1951, p. 180-182):

The north flank of the Alaska Range is characterized by a series of broad eastward-trending folds, formed in middle Tertiary time, that are broken in places by both normal and reverse faults. The pertiary rocks, including the Nenana gravel and the coal-bearing formation, are found in the troughs of the synclines, whereas schists and intrusive rocks older than the coal-bearing formation crop out in the cores of the anticlines. Through the center of the area under discussion passes a major syncline, which is bordered on the north by an anticline and on the south by a tilted fault block that forms the foothill range just south of the mapped area. The syncline, which is 10 to 15 miles wide at the Savage River, probably extends from the 

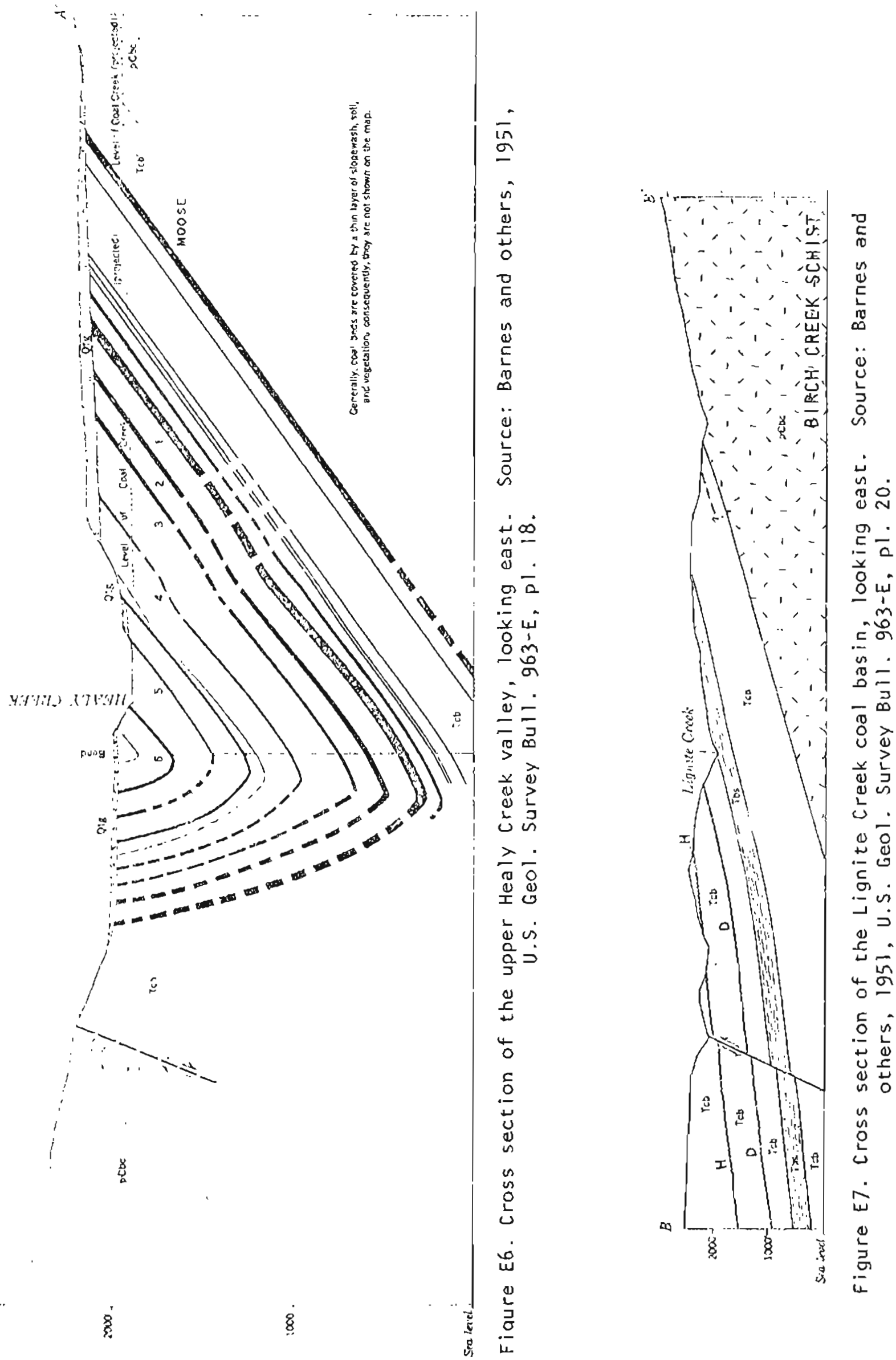
Mustic Croek coal hasin on the east to thi Toklat River on the west, a distance of 80 miles.

\section{Economic Geology}

The Kantishna mining district is the area drained by the Kantishna River and its tributaries. It lies mostly in a group of foothills named the Kantishna Hills, which are underlain by Birch Creek Schist. A number of lode deposits have produced small amounts of gold, silver, antimony, and lead ores from quartz veins. About 1,700 cons of merallic antimony were shipped from the Stampede Mine. Several deposits are within the boundaries of Mount Mckinley Notional Park. The largest of these is on the north slopes of thunt Eielson, where massive argentiferous galena and other sulfides occur in limestone.

A locality of interest from the standpoint of uranium investigations is the Purkey-Pile prospects, just outside the western boundary of Mount Mckinley National Park on the west side of the headwaters of Boulder Creek, a small tributary of the Swift Fork of the Kantishna River. There are three separate prospects within about $3 \mathrm{miles}$ of each other in metamorphosed sedimentary rocks near a small granite stock. The prospects have showings of silver, lead, zinc, and tungsten minerals (Maloney and Thomas, 1966). Six samples from the Mespelt prospect yielded anomalous el values from 0.037 percent to 0.14 percent.

A small deposit of tarry bituminous material originally thought to be a petroleum seep is localed I mile above the mouth of Cripple Creek, which flows northwest into Healy creek. It is in gravels overlying the Tertiary Coal-Bearing Group. Martin (1923, p. 137-147) concluded that the malerial is actually a coal tar produced by discillation from burning coal.

\section{Previous Investigations for Radioactivity}

The writer examined the Tertlary coal-bearing group in the Healy creek valley with feiger counlers (Eakins, 1969, p. 12-13). The area was crossed at several points by walking up gullies so that each bed in the sequence was tested for radioactivity, and foot traverses were made along the entire 12-mile-long area where lihe coal-bearing beds were exposed. The maximum radioactivity in sandstones, shales, and the Birch Creek Schist was about $0.04 \mathrm{mr} / \mathrm{hr}$, or three times the normal background. No radioactivity was detected at the site of the Delta coal mine at the head of ober Creek in the Jarvis coal field (Eakins, 1969, p. 15). Wo other reports of investigations of the Tertiary coal-bearing group on the north flank of the Alaska Range are available. However, it was rumored that a private company was doing streani-sediment and water sampling for uranium in the Healy area last summer (1974), and that uranium anomalies were located.

Radioactivity tests of 50 rock ipecimens collected by the 19478 radFord Hashburn Mount Mckinley expedition produced 0.009 percent ell in a mangan: fercus vein quartz sample; granitic rocks produced as much as 0.004 percent eu (Matzko, 1951; Vedow, 1956, p. 27). Veins in the Mount Eiclson area and in the Kantishna llills area did not produce over 0.001 percent eU (White and others, 1952, p. 7-9: Wedow, 1956, 0. 23). Analyses of samples from the Purkey-Pile Mespelt prospect near the western border of Mount McKinley National Park are listed (Maloney and Thomas, 1966, tabie 3): 


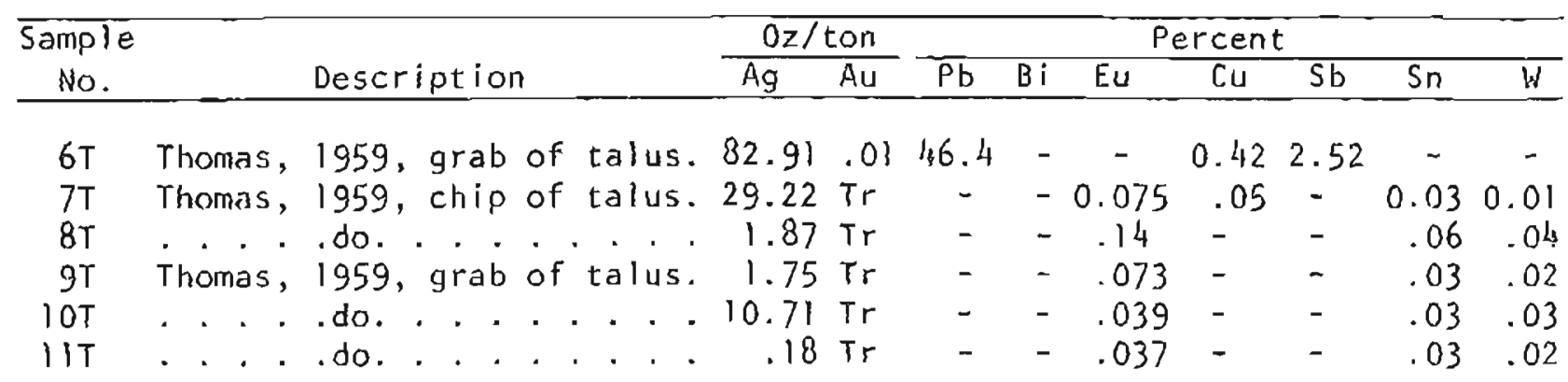

The reported eU values justify an investigation of the area. There has not been any production from the claims, thaugh they have been held since 3921. An old 40-foot shaft on the Mespelt prospect is now caved in. A 7- by 5- by 15-foot shaft on the Jules-knudson prospect was last reported full of water. A little trenching and road work by bulldozer is the only other work that has been done. The prospects are in a remote area of very rugged terrain. 


\section{INVESTIGATION OF ALASKA'S URANIUM POTENTIAL}

Part 2 - Uranium and Thorium in Granitic and Alkaline Rocks in Western Alaska By Bxian K. Jones and Robert B. Forbes

- Geophysical Institute, University of Alaska -

Originally prepared as a Summary Report For the U,S. Energy Research and Development Administration-Grand Junction Office-w. under Contract $\Lambda T(05.1)-1689$ 
Granit: Mountain- - - - - - - - - - 2440

Darby Pluton- - - - - . - - - - - 2- 40

Tane Hills Pluton - - - - - - - - - - 2-43

The Dry Canyon Creek Stack- - - - - - - - 2-47

Selawik Lake Complex- - . - - - - - - 2-50

Conclusions - - - - - - - - - - - - - - 2-52

Mineralogical guidelines for future uranium invest-- -

igations - - - - - - - - - - - - 2-53

Granite Mountain- - - - - - - - - - 2-53

Darby Mountains - - - - - - - - - 2- - 23

Zane Hills- - - - - - - - - - - - 2-53

Dry Canyon Creek Stock- - - - - - - - - - 2-53

Selawik Lake Complex- _ - - . - - - - - 2-54

References - - . - - - . - - - - . - - 2-5k

APPEND!X 1

Mineralogical and chemical data- - - . - - - 2-57 APPENDIX 2

Alkaline igneous racks - - - - - - - 2-61 APPENDIX 3

Examples of correlation coefficients - - - - - - 2-62 APPENDIX 4

Analytical techniques - . . . . . . . . . 2-63

\section{LIST OF FIGURES}

Figure I. Location map, Seward Peninsula and Yukon-

Koyukuk study area - - - - - - - - - - 2-3

2. Locations of plutonic rocks on the Seward

Peninsula and Yukon-Koyukuk Basin which were

examined in this study - - - - - - - - 2-4

3. Classification of the alkaline rocks - - - - 2-7

4. Granitic rock classification - . - . - - - 2-7

5. Geologic and sample locality map of the Granite Mountain Pluton- - . . . . . . . - 2-8

6. Rock modes From the Granite Mountain Pluton- - - 2-9

7. Scintillation counter readings across the west margin of the Granite Mountain Pluton- - - - 2-10

8. Variation in potassium across the Granite Mountain Pluton - - - - - - - - - - - 2-15

9. Geologic and sample locality map of the Darby Pluton - . - - - - . . - . . - 2-16

10. Rock modes from the Darby Pluton - - - - - - 2-17

11. Variation in potassium across the Darby Pluton - 2-18

12. Geologic and sample locality map of the Zane

Hills - - - - - - - - - - - 2-19

13. Rock modes from the Zane Hills - - - - - - 2-20

14. Geologic and sample locality map of the Dry

Canyon Creek Stock - - - - - - - - - 2-22

15. Modes of rocks from the Dry Canyon Creek Stock - - 2-23

16. Location and geologic map of the Selawik Lake

Complex- - _ - _ . . . . . - - 2-25 
Figure 17. Modes of rocks from the Selawik Lake Complex - - 2-26

18. Relative abundance of uranium and thorium within the study area - - - - . . . . . 2-31

19. Log-normal probability plot of uranium concentrations - - - - . . - . - . - 2-32

20. Correlation matrix for 108 samples analyzed in this study - . . - . . - . . - 2-37

21. Correlation matrix for 32 samples from the Granite Mountain Pluton- - - - - - - - 2-41

22. Variation in uranium, thorium, potassium, and counts per second across the Granite Mountain Pluton _... . . . . . . . . . . . 2- 42

23. Correlation matrix for 22 samples from the Darby Pluton - . . - . . . . . . - 2-44

24. Variations in uranium, thorium, potassium, and counts per second across the Darby Pluton- - - 2-45

25. Correlation matrix for 32 samples from the Zane Hills Pluton - - . - - - - - - - 2-46

26. Plot of quartz against thorium concentrations from the Zane Hills Pluton- _ . . . . . . 2-47

27. Variation in uranium, thorium, potassium, and counts per second along two traverses in the Zane Hills Pluton- - _ . . . . . . - 2-48

28. Correlation matrix for 16 samples from the Dry Canyon Creek Stock - - - . - . . . 2-49

29. Correlation for 10 samples from the Selawik Lake Complex - - . - . - . . . 2. - 2.51

30. Correlation coefficients - - - - - - - - 2-62

31. Number of thin sections required to maintain major inineral analvtical error less than $1.41 \%$, $2.0 \%$, and $2.5 \%$, based on the area of the thin section and the $1 \mathrm{C}$ number- _. . . . . 2-66

\section{LIST OF TABLES}

Table I. Modal percentages of the franite Mountain Pluton nepheline syenite- - . . . . . . - 2-10

2. Modal percentages of the Granite Mountain Pluton garnet syenite - - - - - - - - . - 2-11

3. Modal percentages of the Granite Mountain Pluton monzonite - . . _ _ . . . . . . 2- 2-12

4. Chemical analysis of monzonite from the Zane Hills - . - - - . - - . - - - 2-2l

5. Means, standard deviations, maxima and minima for uranium and thorium data for five plutons in western Alaska - . . . . . - . - 2- 29

6. Uranium concentrations in mineral separates and estimation of the contribution of individual mineral phases to the whole rock uranium content - . . . . . . . . . . - 2-33 
Table 7. Means, standard deviations, and maxima and minima of uranium and thorium data, by rock type - - 2-38

8. Summary table for the analysis of varianceuranium - - . - - - . - - - - 2-39

9. Replicate analyses of DC-5-1 - - - - - - 2-64

10. Replicate analyses of mineral separates - - - 2-64 


\section{ABSTRACT}

One hundred eight rock samples were selected from traverses across five plutons in western Alaska and analysed for uranium, thorium, and potassium. Modes were determined using point-counting techniques. The highest uranium concentrations detected were 36 and 92 ppm from a mineralized dike intrusion zone in the Selawik Lake Complex.

Correlation coefficients were calculated in an attempt to relate the inineralogy to uranium and thorium content. When the sample set is considered as a whole there is little significant carrelation. However, the analysis of individual plutons yields strong correlations between mineralogy and radioactivity. The mineralogical variable that correlates with uranium or thorium varies from one pluton to the next.

Based on these correlations, mineralogical guidelines are offered for the selection of uranium enriched variants in four of the five plutons.

\section{INTRODUCTION}

\section{Project Objectives}

With increasing interest in the location and development of new uraniuri reserves, the state of Alaska has become a prime target area for exploration. Most of Alaska has been geologically mapped at the reconnaissance level and to date uranium investigations have been limited. franitic and alkaline rocks on the eastern Seward Peninsula, and large granitic plutons and alkaline stocks in the Hogatza Plutonic belt of the Yukon-Koyukuk Basin have been known to contain high concentrations of uranium and thorium, since early prospecting efforts (fiault et al., 1953, West, 1953, and West and thite, 1952).

In 1975 Gilbert R. Eakins of the State of Alaska reological and fieophysical Surveys received a contract from the U.S. Energy Research and Development Administration (ERDA) to conduct an extensive bedrock and stream sediment sampling investigation of this region. This thesis represents a significant portion of the hard rock section of the investiga$t$ ion headed by Dr. Robert B. Forbes of the Geology Department and Geophysical Institute, University of Alaska.

\section{Previous Work}

Moffit (1905) was the first to note the existence of large calcalkaline plutons and smaller stocks in western Alaska. He also documented the occurrence of melanite garnet and aegerine at Granite Mountain. Smith and Eakin (1911) reported nepheline-bearing veins in the Darby Mountains. The alkaline complexes in the Kobuk-Selawik lowland were discovered and first discussed by Patton and Miller (1963) (see also Patton and others, 1968; and Miller, 1972).

Uranium investigations began in the area in the late 1940's, as documented by the publication of a number of U.S. Fieological survey Circulars by West (1953), West and thite (1952), and fiault and others (1953). 
More recent interest in the uranium notential of the region has resulted in o number of hard-rock uranium investigations (Miller and Bunker, 1975a and b; Milier, 1975; Stantz and Miller, 1976). A definisive study of the petrology of the Hogatza Plutonic belt was conducied by Miller (1970), and a more specific study of the alkaline compicxes was also published by Miller (1972).

Although the common association of anomalously high values of uranium and thorium with rocks containing feldspathoids is well documented, high concentrations of the radioactive elements are not limited to the alkaline variants alone. The Darby Pluton in the southeastern Seward Peninsula is an example of a particularly uraniferous pluton (Miller and Bunker, 1975a, and Miller, 1975) although it is composed chiefly of a silicic quartz monzonite. The occurrence of above average amounts of uranium and thorium in plutonic rocks that range in age over a period of at least 35 million years, that have a composilional range from biotite granodiorite to nepheline syenite, and are in significantly different geologic settings suggests the existence of a uranium-thorlum province (Miller, 1975).

\section{rieologic Setting}

Alkaline and calc-alkaline igneous rocks occur in a belt approximately $750 \mathrm{~km}$ long in western Alaska. This belt of plutons extends across the northern Yukon-Koyukuk province from the Koyukuk River to Kotzebue Sound, where it is called the Hogatza Plutonic Belt; cuts across the eastern Seward Peninsula, and may include intrusives on St. Lawrence Island and eastern Siberia.

The rocks along this zone are predominanlly granodiorite and quartz monzonite, with some syenite, monzonite and granite (Miller, 1970). Associated with the larger plutons are a number of small, highly potassic alkaline stocks (Miller, 1972). A number of these plutons have been sampled in the past and were found to contain anomalous concentrations of uranium and thorium, but little systematic exploralion has been done.

The Hogatza Plutonic Belt is intruded into the Yukon-koyukuk province, an area of late Mesozoic andesitic rocks, volcanic graywacke and mudstone. It has been a highly mobile tectonic province which has been subjected to repeated magmatism during Cretaceous and Tertiary time (Pation, 1970a).

Precambrian rocks of the Seward Peninsula consist chiefly of gneiss, pelitic and chloritic schists, and carbonate rocks. The Paleozoic Era in the Seward Peninsula is dominated by carbonates. These rocks have been imbricately thrust faulted to the east and north during two periods of faulting in Cretaceous time (Sainsbury, 1963). The boundary between the Seward Peninsula and the Yukon-Koyukuk province appears to be a thrust fault, with Paleozoic carbonate rocks chrust eastward over Cretaceous volcanic rocks (Patton, 1967, Sainsbury, 1369). K/Ar age dates indicate that crystallization of the plutons in the western Rogatza Plutonic Belt occurred about 100 million years ago (Miller, 1970). Radiogenic ages and spatial relacions suggest that the alkaline rocks 
are co-magmatic with the larger monzonite, syenite, and quartz monzonite plutons of the western Hogatza plutonic belt (Miller, 1970). Ages in the eastern section of this belt appear to be much younger, about 86 million years.

\section{Field Investigations}

During the 1975 field season, 108 rock samples were collected from two large plutons and three small stocks on the eastern Seward Peninsula, and the logatza Plutonic Belt. These plutons include the Darby Pluton, Zane Hills Pluton, Selawik Lake Complex, Dry Canyon Creek Stock, and the Granite Mountais Pluton.

The plutons were selected for sampling because of previously reported occurrences of anomalously high concentrations of radioactive elentents. Locations maps for this study are shown in Figures 1 and 2.

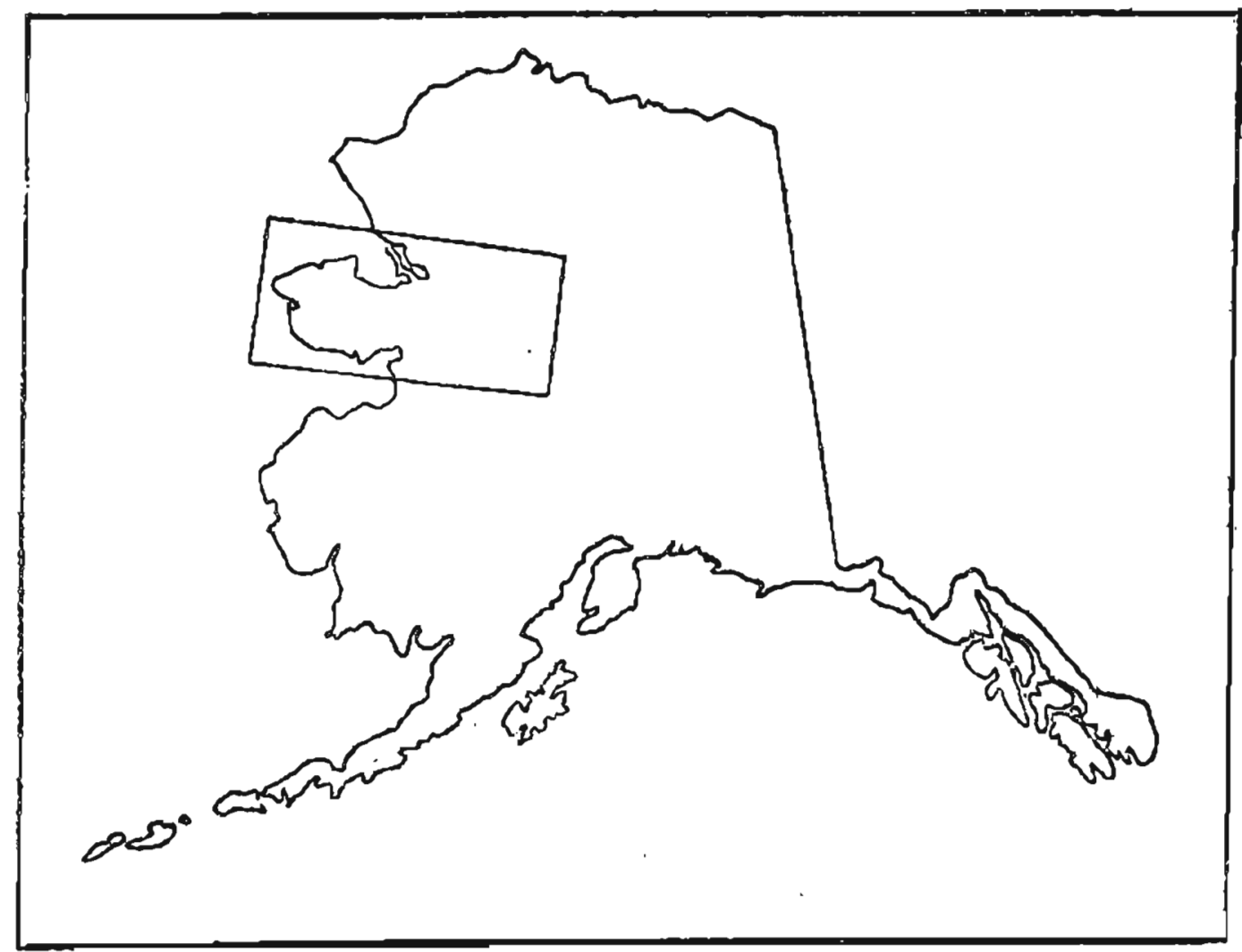

Fiqure I. Localion map, Seward Peninsula and Yukon-koyukuk study area. 


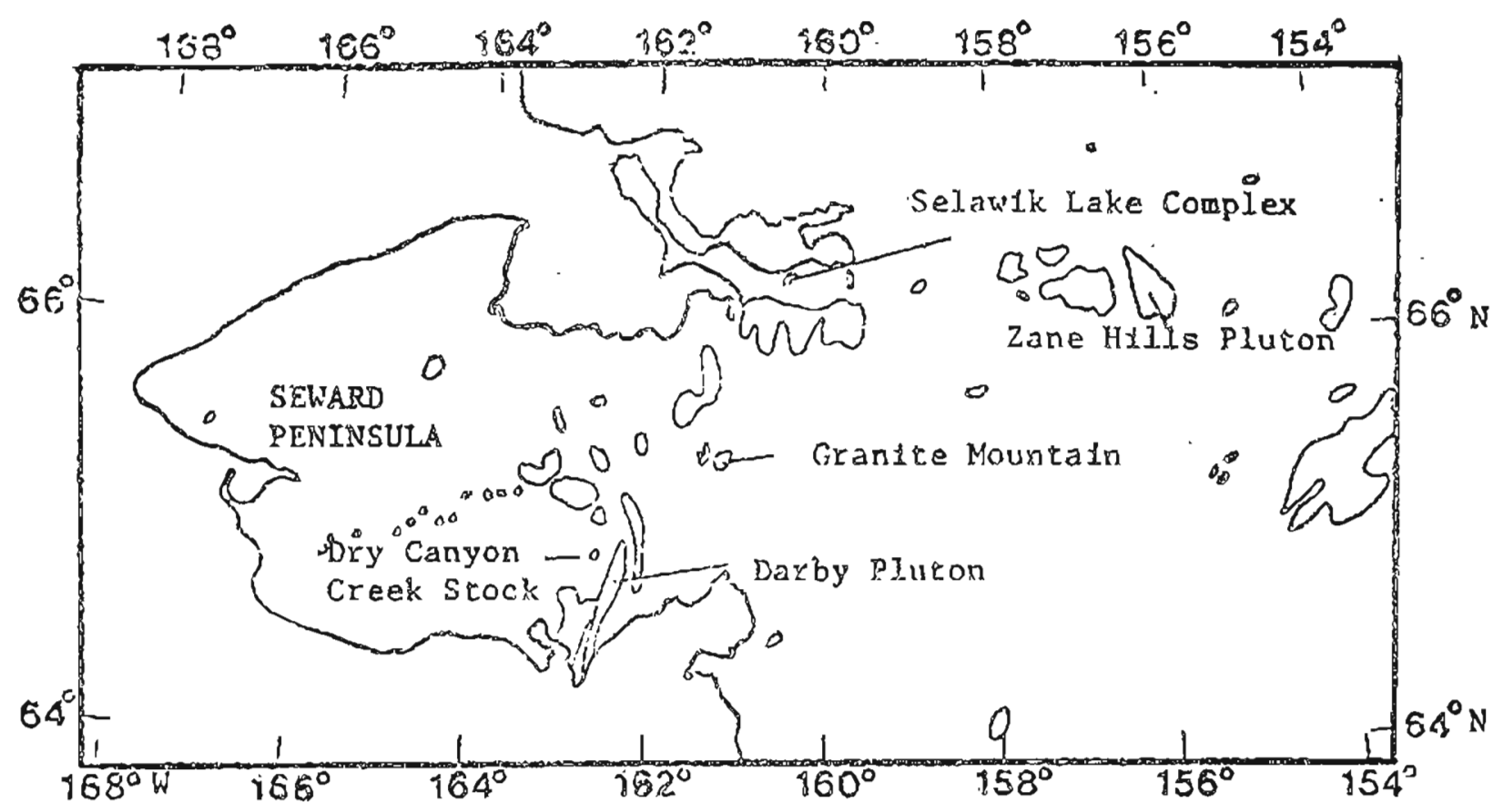

Pigure 2. Locations of plutonic rocks on the Seward Peninsula and Yukonknyukuk Basin which were examined in this study.

\section{Cranile Mountain}

This small stock was selected for study because it is a zoned alkaline intrusive (Miller, 1972). It seemed to be the best possible locality within the thesis area to study the distribution of uranium and thorium in rocks showing systematic variation in their modes. Although outcrops are sparse at Granite Mountain, near continuous rubble crop occurs along the traverse line, and rock samples were collected at intervals of approximately 300 meters.

Darby Pluton

The Darby Pluton is a long narrow quartz monzonite intrusive known to contain anomalous concentrations of uranium and thorium. Analyses of 11 samples reported by Miller and Bunker (1975a) yielded average values of $11 \mathrm{ppm}$ uranium and $59 \mathrm{ppm}$ thorium. Because of its large size, and the availabie time, it was not possible to sample all of the pluton at close intervals. As a result, a single east-west traverse was completed in an area of known high radioactivity. This area is in the vicinity of Vulcan Creek, $33 \mathrm{~km}$ north of the village of El im, where Hest (1953) discovered high concentrations of uranium in pan concentrates, and $\mathrm{Miller}$ and Bunker (1975a) collected three rock samples that averaged $16 \mathrm{ppm}$ uranium. Outcrop and rubble crop are abundant along this traverse and samples were taken at intervals of approximately $.5 \mathrm{~km}$.

Zane

Two sample traverses were made through the Zane Mills. A northern traverse was conducted through an area of granodiorite known to contain 
rather low concentrations of uranium and thorium. The purpose of this traverse was to determine the mineralogy associated with uranium poor rocks and to examine the variability in such a pluton, where the mode is consistent.

A second traverse was conducted in the southern Zane Hilis, through two zones of monzonite known to contain anomalously high concentrations of uranium and thorium. Dutcrop was abundant in both of these traverses. Samples were taken at intervals of about $1 \mathrm{~km}$.

Selawik Lake

The Selawik Lake Complex is a small potassic stock located north of the Selawik Hills. It was known to contain uranium concentrations as high as 6. ppm and thorium concentrations of 22. ppm. Outcrop is poor and most of the sampies collected came from the rubble crop.

\section{Dry Canyon Creek}

The Dry Canyon Creek Stock, located on the west side of the Darby Mountains is a potassic stock containing high concentrations of thorium but low uranium values. Most of the samples were taken from the rubble crop.

Analyses from the Selawik Lake Complex and the Dry. Canyon Creek Stock were taken to see where the uranium is concentrated in these rocks and with what types of minerals it is associaled.

\section{RE.SEARCH IJBJECTIVES AND FINDIIISS}

\section{Proposed Objectives}

1. To gain additional information of the mineralogy and petrology of the alkaline and alkalic rocks within the study area.

2. To examine carefully areas of anomalous radioactivity.

3. io determine which minerals in the rock contain uranium and thorium, and the distribution of these elements between major, minor, and accessory mineräls.

$i_{i}$ To make correlations between mineralogy and the uranium concentrations found in the rocks.

5. To predict which rock types would be expected to contain high conccentrations of radioactive minerals.

6. To compare airborne gamma-ray spectrometry data with analyses of rock samples collected along traverses beneath the flight $\mathrm{l}$ ines.

Findings

1. Petrographic analys is of alkaline rocks revealed a number of minerals that had not been previously described in the thesis arca. In 
addition, the textures and structures of these rocks were described in greater detail than they had been previously. Additional modal analyses were determined from the plutons examined and a number of interesting field relations and now plutonic units were discovered.

2. Areas of anomalous radioactivity were studied, and rocks were collected that contained uranium values as high as 92 ppm.

3. Uranium analyses of mineral separates plus the careful study in thin sections for those minerals showing pleochroic haloes, indicate where uranium and thorium are concentrated in the rock.

4. Correlations between rock types, major and minor minerals, and uranium and thorium were made using graphical and basic statistical techniques.

5. Predictions were made on the basis of the above determinations and mineralogical guides suggested for the selection of samples that should contain anomalous uranium and thorium.

6. Comparison between ground-truth and airborne data could not be made because of the coarse interval selected for the listing of the airborne analytical data published by rexas Instruments, the contractors for the airborne study, and the assumptions made during the processing of that data concerning the geology. It appears that the techniques used, particularly the corrections for known geology are detrimental to the usefulness of these surveys.

\section{ROCK CLASSIFICATION}

The classification scheme used for the alkaline rocks in this study is shown in Figure 3. This comparatively simple scheme was presented by Sorensen (1974) as taken from Sarantsina and Shinkarev (1967). Rock names are assigned on the basis of three constituents; percent nepheline, percent alkali feldspar, and percent mafic minerals. The most significant advantage of this particular chart is that it minimizes the number of unussal names given to alkaline rocks. Abundant rock names greatly restrict the audience that would be able to comprehend easily the subject matter of a petrologic report. A large field of nepheline syenite exists within the diagram. This is where most of the rock modes studied fall. Mineralogical modifiers are used to describe the particular variety of nepheline syenite.

The Plagioclase-K-feldspar-Quartz classification chart as used in this study for name assignments for granitic rocks is shown in figure 4.

\section{THE ROCKS}

\section{Granite Mountain}

As mapped by Miller (1972), the franite Mountain Pluton covers an area of approximately $70 \mathrm{~km}^{2}$. It is a zoned alkaline-calc-alkaline stock with nepheline syenite and garnet syenite at the outer rim, surrounding a unit 


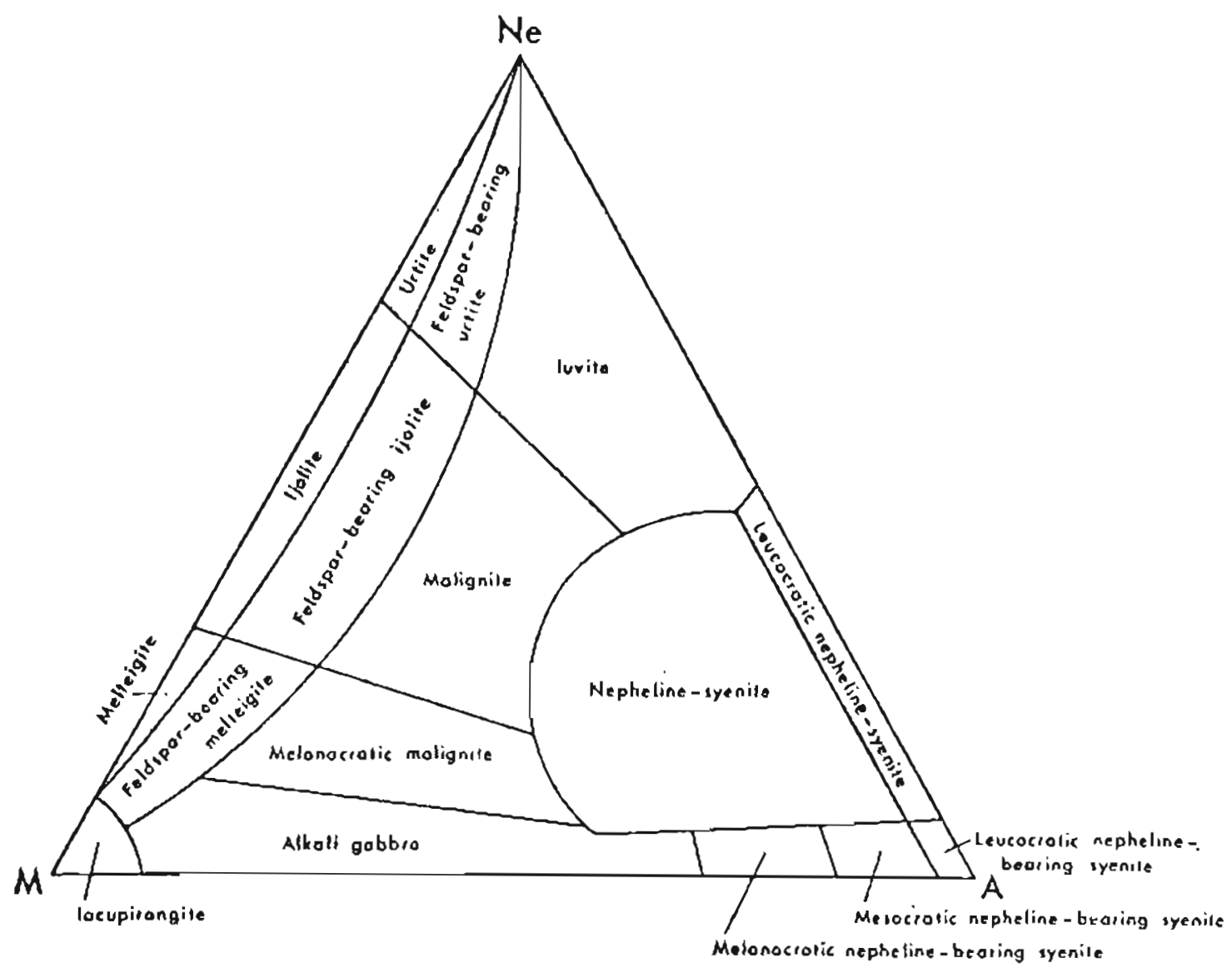

Figure 3. Classification of the alkaline rocks.

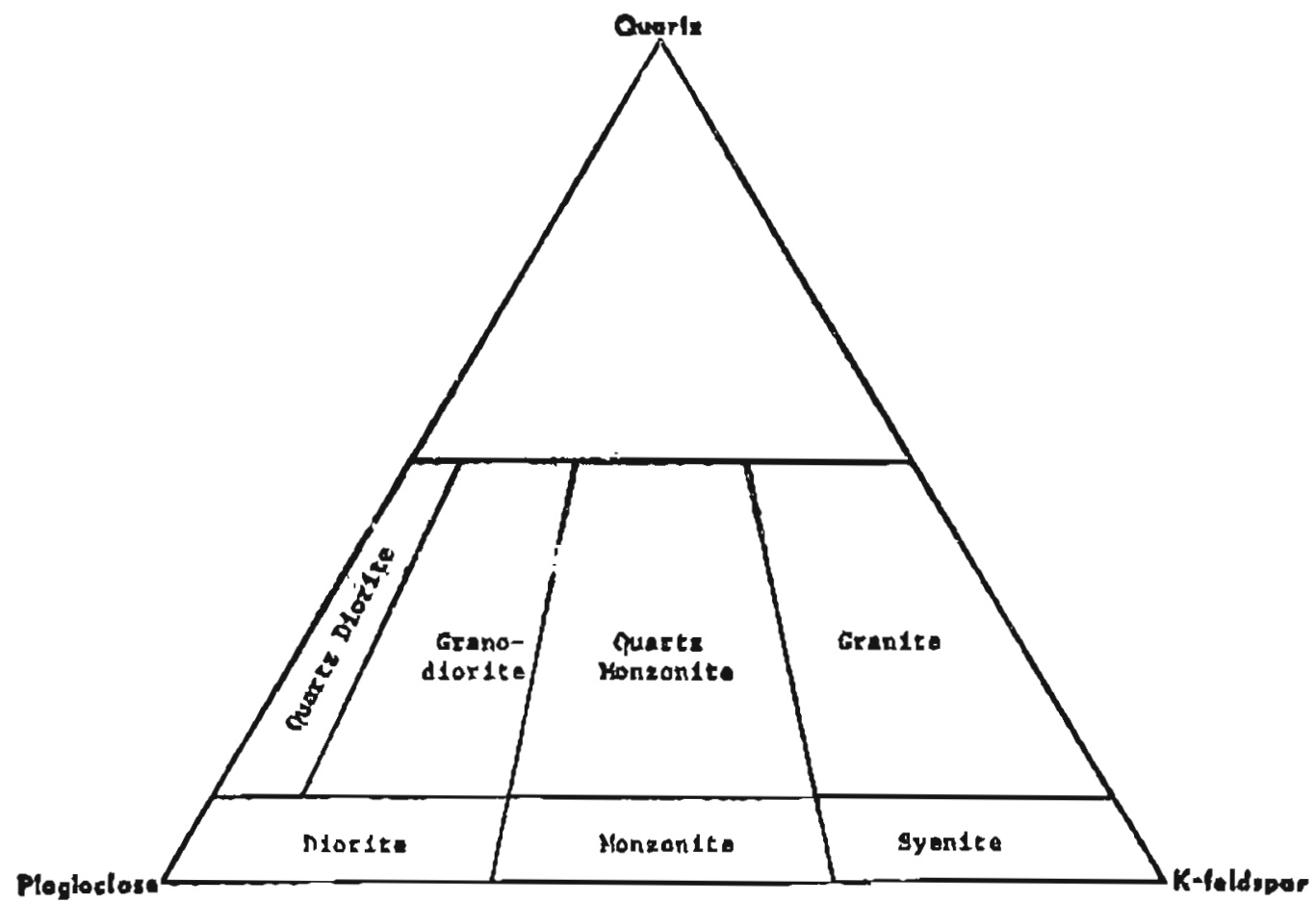

Figure 4. Granitic rock classification. 


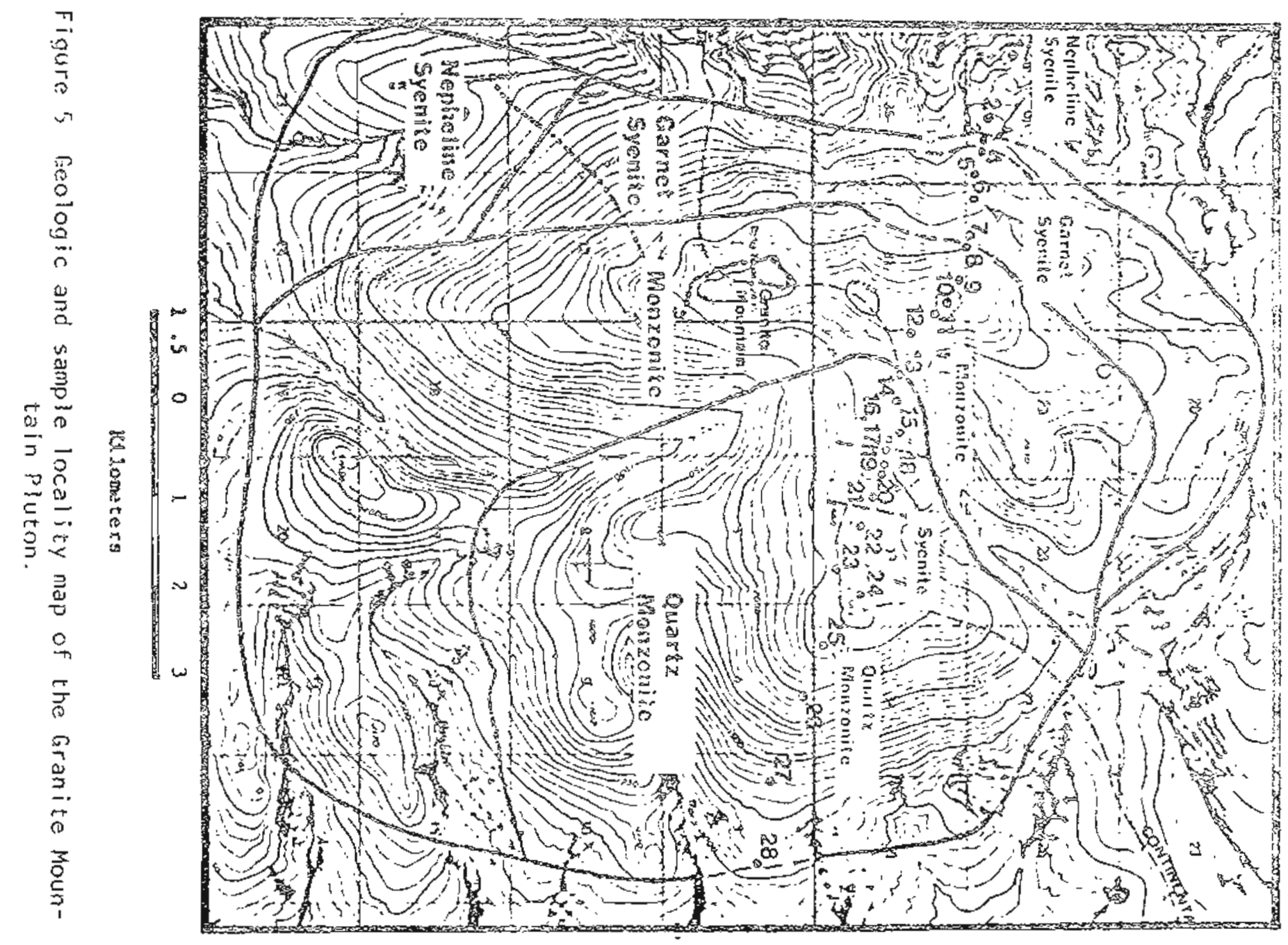


In the figure, solid lines and rock names in large print represent the geology as taken from Miller (1972). Heavy dots are sample localities from the present investigation and the dashed lines and rock names shown in smaller print are contacts and rock types as determined from petrographic examination of these samples. Rock modes, as determined in this study, are shown in Figure 6 (see Appendix A for point-counting techniques). There are major differences between the geology as determined by Miller (1972) and what we determined during our detailed traverse.

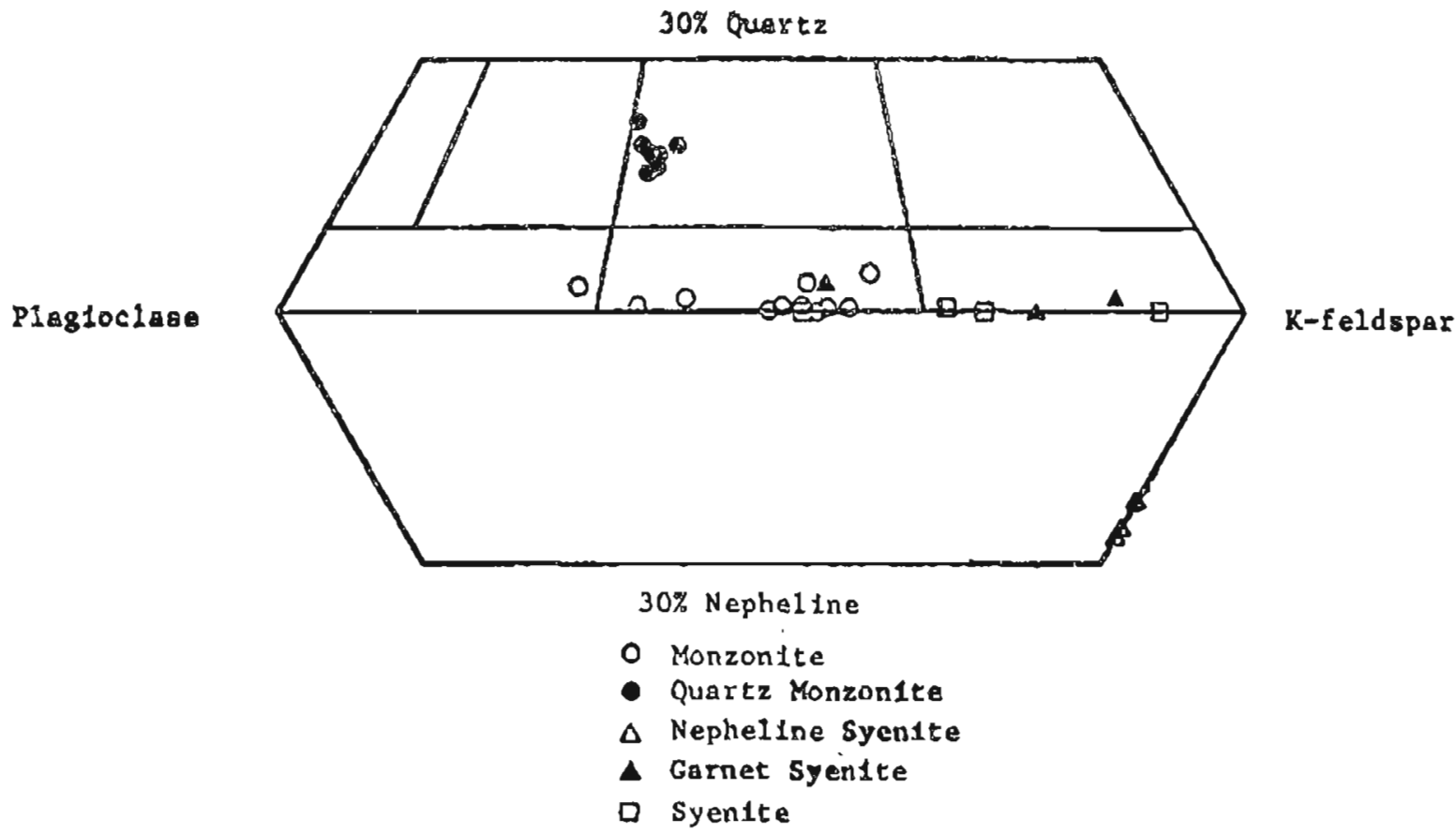

Figure 6. Rock modes from the Granite !ountain Pluton.

Contact Relations

As seen from aerial photographs a distinct lineament is coincident with the west boundary of the Granite Mountain Intrusive. Andesite breccia was found at location 2, Figure 5. There is little or no evidence of recrystallization of the andesitic country rock adjacent to the intrusive. These observations suggest that the western contact is a fault.

The eastern contact is covered by tundra and its nature in the vicinity of the traverse was not determined.

The exact location of the western contact along the traverse was fixed with the use of a scintillation counter (Figure 7). Although the area is covered by tundra, the location at which the scintillation count begins to increase is also marked by a distinct vegetation change. The contact has been mapped at this vegetation change. 
A newly recognized nepheline syenite unit is inferred at sample locality 3 becalise of the discovery of relatively abundant nepheline syenite float. This sample locality is located on a broad saddle, with andesitic rocks to the west and syenite and monzonite to the east. The only likely source for the nepheline syenite float is the bedrock beneath the tundra-covered saddle. Making this assumption we are able to map a thin unit of nepheline syenite.

The modal composition of the nepheline syenite as determined by point counting three rock samples is shown in Table 1.

Table 1

Modal Percentages of the Granite Mountain Nepheline Syenite

\begin{tabular}{lccc}
\multicolumn{1}{c}{ Mineral } & Mean & Standard Deviation & Range \\
\cline { 4 - 5 } Nepheline & 18.6 & 15 & $16.5--21.2$ \\
Orthoclase & 56.3 & 3.12 & $52.8--58.7$ \\
Aegerine & 5.6 & 1.42 & $4.3--7.1$ \\
Biotite & 4.3 & .6 & $3.6--4.8$ \\
Garnet & 11.3 & 5.9 & $7.5--18.3$ \\
Apatile & trace & & \\
Orthite & trace & & \\
Opaques & trace & & \\
Zeolites & trace & & \\
Cancrinite & trace & &
\end{tabular}

The nepheline syenite has a medium-grained hypidiomorphic granular texture with no mineral foliation. However, there is evidence of shearing and cataclasis between large orthoclase grains.

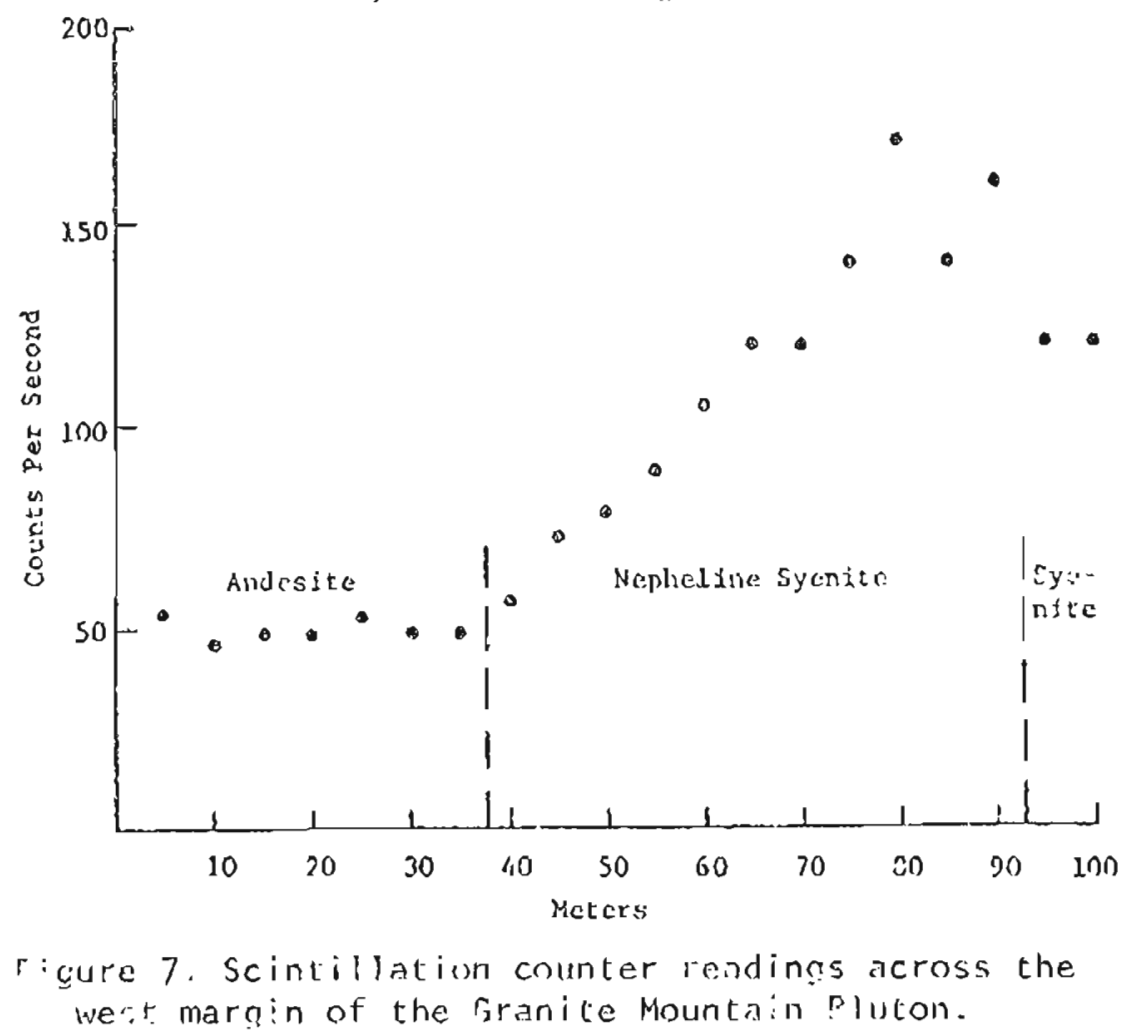


Perthitic orthoclase occurs as subhedral grains up to $5 \mathrm{~mm}$ in length. Nepheline is interstitial in the feldspar fabric, and can be distinguished in the outcrop hacause it develops negative relief on weathered surfaces. In thin section, the mepheline can be distinguished by higher relief than feldspar: most grains show some form of alteration to cancrinite, clay minerals or zeolites.

Aegerine is deep green, occurring in subhedral grains with an optic angle of $60^{\circ}(-\cdot)$. Euhedral dark-reddish brown melanite garnet, a titaniumrich form of andradite, is also abundant.

The contact with the garnet syenite to the east cannot be observed because of the tundra cover. Miller (1972) helieves that the contacts within the zoned intrusive are sharply gradational. The existence of similar looking malanite garnets in the two units suggests a genetic interrelationship.

\section{Garnet Syenite}

The garnet syenite unit is characterized by porphyritic and gneissic textures. Orthoclase feldspar, constituting as much as 60 percent of the rock occurs in large dull-white colored crystals up to $5 \mathrm{~cm}$ in length.

The garnet syenite unit as shown in Figure 5 has been mapped more in terms of texture than mineralogy. The mode varies substantially within the unit and half of the rocks examined in this study were monzonites. The distinctive porphyritic and gneissic textures found in the unit are in sharp contrast to the fine-to-medium-grained subhedral textures of the monzonite and quartz monzonite to the east.

Miller's (1972) petrographic modal summary of this rock unit is given in Table 2.

Table?

Modal Percentages of the Granite Mountain Garnet Syenite (after Miller, 1972)

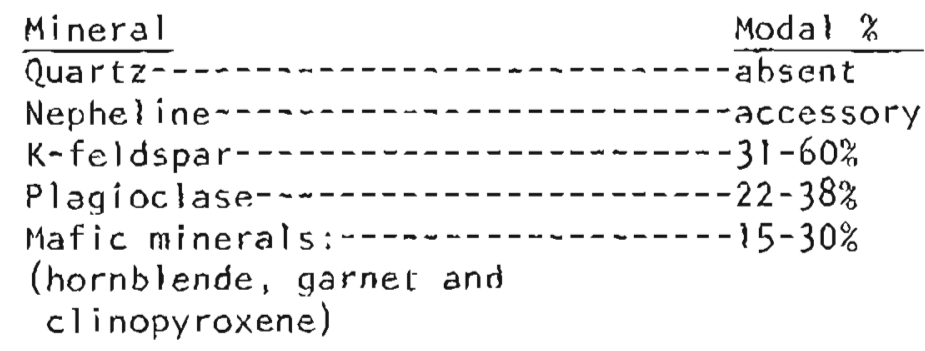

In the petrographic examination of six samples from 4 localities within the unit, the modal percentages fell within the limits determined by Miller, with the exception that quartz was found in all sections in minor amounts.

Accessory minerals include large euhedral sphene crystals, apatite, and traces of secondary carbonate. 
The pleochroic scheme of the amphibole is: $x=b l u i s h$ green, $y=d e e p$ green to black, $z=l i g h t$ yellowish green, suggesting high concentrations of $\mathrm{Na}$ and $\mathrm{Fe}$. The amphibole appears to be in the eckermannite-arvedsonite family of amphiboles, a group of amphiboles characleristic of alkaline igneous rocks and their associated pegmatites (Deer and others, 1963).

Monzonite

Table 3 shows percentages of mineralogy of 10 thin sections and rock slabs from the monzonite unit at Cranite Mountain.

Table 3

Modal Percentages of the Granite Mountain Monzonite

\begin{tabular}{lccc}
\multicolumn{1}{c}{ Mineral } & Average Mode & Std. Dev. & Range \\
\cline { 2 - 3 } Quartz & .42 & 0.48 & $.1-2.9$ \\
Plagioclase & 39.9 & 7.2 & $32.7-55.8$ \\
K-feldspar & 44.9 & 7.6 & $23.4-57.6$ \\
Amphibole & 7.4 & 4.2 & $2.5-13.4$ \\
Pyroxene & 5.4 & 2.3 & $2.2-13.9$ \\
Sphene & 0.5 & .3 & $.1-1.2$ \\
Apatite & .5 & .2 & $.1-.9$ \\
Opaques & .6 & .3 & $.1-1.3$
\end{tabular}

The monzonite is generally equigranular showing no distinctive planar foliation. Grains average $1-3 \mathrm{~mm}$ in size and often show evidence of shearing and cataclasis along their margins. Although this is the predominant cexture, a few samples of a coarser monzonite were collected in the unit Lhat showed gneissic and porphyritic lextures similar to those found in the garnet syenite unit. The existence of these samples suggests that the granitic texture in the monzonite is the result of grain size, and that the monzonite may have been sheared by the same process that effected the outer syenite unit.

Plagioclase An $15-28$ occurs in zoned, largely untwinned crystals .5-3 $\mathrm{mm}$ in size with altered calcic cores. it is locally antiperthitic. Kfeldspar is very perthitic and occurs in subhedral crystals. Quartz is not abundant and is interstitial to the feldspars.

Amphibole and aegerine-augite are the major mafic constituents, comprising as much as $17 \%$ of the rock. Although chemical analyses are not available for this rock unit the existence of aegerine-augite suggests that the ratio of alkalies to silica may exceed $1 / 6$, that necessary to classify this rock as alkaline (see Appendix 2).

Sphene and apatite are abundant in most of the thin sections. Other accessories include fluorite and zircon.

Syenite

East of the monzonite unit, a zone of pyroxene hornblende syenite, similar cexturally to the garnet syenite unit was discovered. The syenite 
un!t was discovered. The syenite unit is $.75 \mathrm{~km}$ wide along the traverse i ine. Although it was not mapped throughout the pluton, our reconnaissance of the zone in a north and soush direction revealed that the unit persists for at least 500 meters.

The syenite unit as shown in rigures 5 and 6 consists of a number of rock typcs cxhibiting a characteristic gncissic and porphyritic texture, dominated by coarse grains of $\mathrm{K}-\mathrm{fe}$ idspar in a natrix of plagioclase and mafic minerals.

Of tho zix samples examined from four localities within the syenite unit, four of these rocks are actually syenite, the remaining two are monzonite. Leucucratic quartz monzonite vein-rocks were also found.

Pertaitic orthociase in subhedlal, aligned, grains is the predominant mineral within the syenite unit, constituring up to $91 \%$ of the rock.

Piagioclase An 18-23 is subhedral and largely interstitial to the K-feldspar. Much of it has been altered to sericite. Traces of fine-grained interstitial quartz were found in all of the thin sections studied.

The composition of the amphibole is variable as indicated by a variety of plenchroic schemes seen in different specimens. In some of the variants the amphiboln: is a brown hornblende; other samples show the blue-green pleochroism indicative of Na-rich amphiboles.

The pyroxene is zoned aegerine-augite, displayinn a light greenish color in the center of the crystals, the color growing more intenst towards the rim.

Some secitions show indications of late stage introduction of silica a a b"ie " " smali veins of myrmekitic intergrowths. Cataciasis is evident,

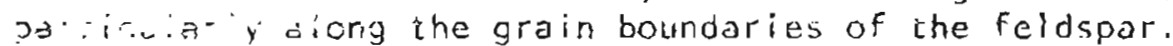

Yitarism concentrations for the two syenite units in the firanice Mountain pluron are almost identical. A chemical analysis of a sample of the garnet syenite yielded $0.59 \%, \mathrm{FiO}_{2}$ ( $\mathrm{Millier}, 1972$ ). The titanium content of the syenite unit, as determined through point counting analysis, is $0.40 \%$. Additionai accessory minerals include apatite and zircon.

A!though the contact between the monzonite and syenite units could not be seen because of the absence of outcrop, the lithologic change was observed to occur over an area of a few meters suggesting a sharp contact.

\section{Ruartz Monzonite}

The contact between the syenite unit and the quartz monzonite occurs in a tundra covered saddie (Figure 5), and the exact location was not pin pointed. Texturally the quartz monzonite is quito different from the syenite units, but is similar to the monzonite to the west.

Sever samples were collected rom the quartz monzonite unit. The nodes of these samples are quite similar, as are their texturai features (:igure 6). 
The quartz monzonite is medium-grained and roughly equigranular. The texture is characterized by plagioclase mantled by large microcline crystals in optical continuity. Presumably the liquid magma became depleted in sodium and calcium during crystallization and the plagioclase became unstable. The magma, now enriched in potassium began to crystallize K-feidspar around the crystallization sites initiated by the sodic plagiociase. All of the granitic uranium-bearing rocks of the eastern seward Peninsula and northern Yukon-Koyukuk basin examined in this study display this unusual texture.

The most abundant mineral phase is plagioclase, An25-32: It is normally zoned with altered cores. Microcline is very perthitic. Interstitial quartz constitutes as much as $21 . \%$ of the rock. The abundance of quariz afpears := increase towards the east.

ine arpolie has a slight greenish tint, and in some cases is seen rimming biotite crystals. A few small augite crystals were observed. Biotite is found is some samples, partially altered to pennine. Accessory minerals include sphene, apatite, and traces of minute zircon crystais.

Although the quartz monzonite does not display a gneissic fabric in hand specimen, on the microscopic scale there is definite evidence of cataclastic deformation which occurred largely in the solid state. Granulation as the result of shearing has occurred along feldspar boundaries. in addition quartz grains commonly show wavy extinction. All of the zones in the Granite Mountain Pluton appear to have been subjected to cataclastic deformation, but because of their different average grain sizes during the cataclastic event, each unit has responded differently. Examination of the intergranular boundaries suggests that the quartz monzonite has been subjected to shear stresses similar to those that deformed the syenite unit to the west. The persistence of the granitic texture is a result of the smaller grain size and the absence of elongated feldspar laths.

Alterstion in the feldspars, pennine replacing biotite, and the occlyarife $a^{2}$ amphibole rimming blotite suggests that the quartz monzonite has ifan elec.ed by a perlod of thermal metamorphism.

Potassium Analyses

Whole rock potassium analyses were made of variants from the firanite Mountain Pluton using atomic absorption spectrometry. These data are shown graphically in Figure 8 . A general decrease in potassium occurs towards the east, disturbed by a number of major fluctuations, particularly within the syenite units.

Comments on the Genesis of the franite Mountain Pluton

The existence of a similarly zoned alkaline complex at Cape Deshnev in siberia suggests that the zone is not the result of anatexis of the country rock (Miller, 1972). Miller suggests that the zoning is the result of multiple injection by a magma, with the initial composition of a leucite prophyry that has separated into two magmas and differentiated, forming the four major rock types at Granite Mountain. Miller notes that such a differentiation scheme has been suggested by Fudali (1963) and Bowen (1928) for similar zoned alkaline complexes. 


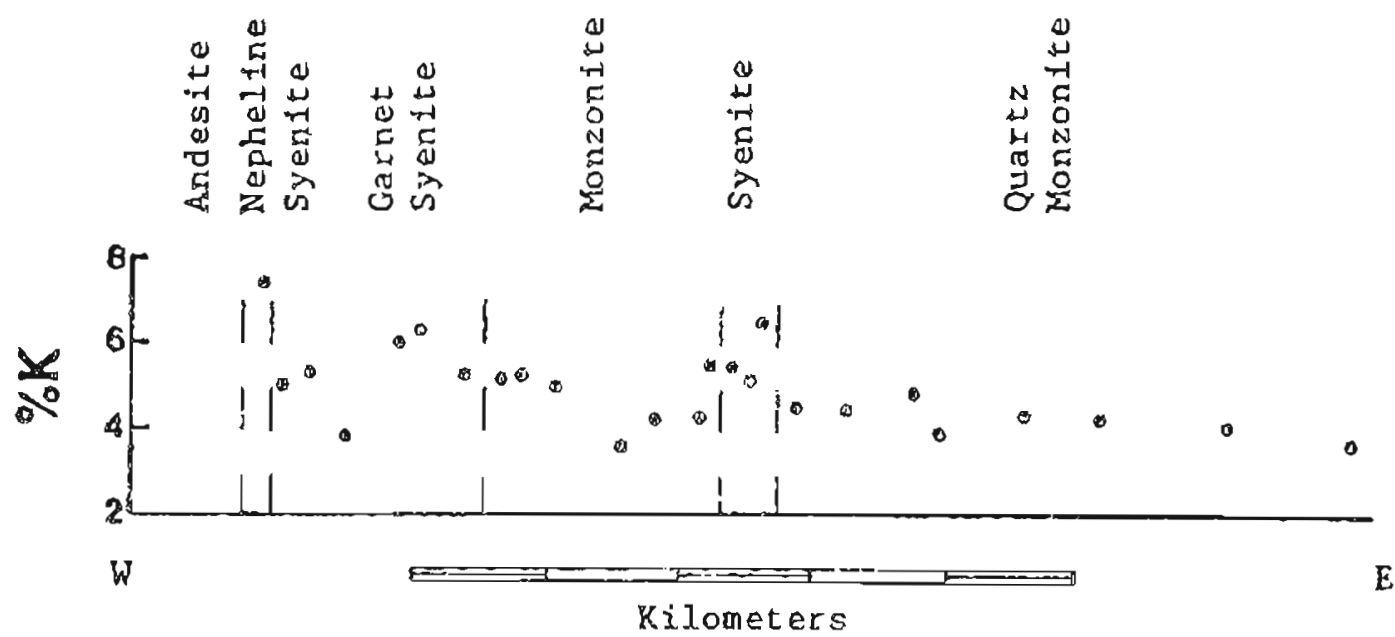

Fiqure 3. Variation in potasalun: veross the Granlle Moumlitin pleton

The :xistence of the additional syenite unit adjacent to the quartz monzonile at Granite Mountain makes models for the origin of this stock even more difficulc. The study of the geology along this traverse in detail wogests that the zoning is much more complex than was believed by Milie:r (1972) during his initial reconnaissunce. In addition, the conLinuous increase in quartz. Fronl west to east sungesls lhat we are only observing half of the pluton. Before the crystallizalion history can be determined, detailed gcologic mapping will have to be conducled over the entire pluton. At present the nature of the contacts is largely speculat ive. The absence of actual outcrop fiakes the determination of contact relations difficult, and the complete lichen cover makes geologic investigations redious.

\section{Darby Pluton}

The Darby Mountains form a prominent north-trending mountain range in the eastcrn Seward Peninsula, exiending From Cane Darby to the Bendeleben Mountains, a distance of about $85 \mathrm{~km}$. The Darby Pluton, a sinuous quartz monzonite body, comprises the cast side of this mountain range. In sharp contrast to the Granite Mountain intrusive, the Darby plutor is enriched in silica and contains as much as $32 \%$ quartz.

Hign uranium values from the Darby Mountains were discovered in pan concentrates of strcam sediment samples by Gault and others (1953). Miller and Bunker (1975a) conducted a hard rock sampling survey of the Darby Pluton, the adjacent Kachauik Pluton and the Bendeleben Pluton.

An east-west traverse approximately $12 \mathrm{~km}$ in length, was conducted during this study across the Darby Pluton in the vicinity of Vulcan creek. In the area of the traverse, the western edge of the pluton is in intrusive contact with low-tn-medium-grade metamorphic rocks of precambrian age (Miller and others, 1972) on the cast side, the Darby Pluton is in fault contact with Devonian dolomite.

Thirty rock samples were collected from the 22 lacalities shown in Figure 9. There is considerable variability in the mode across the traverse as indicated by the scatter in Figure 10. 


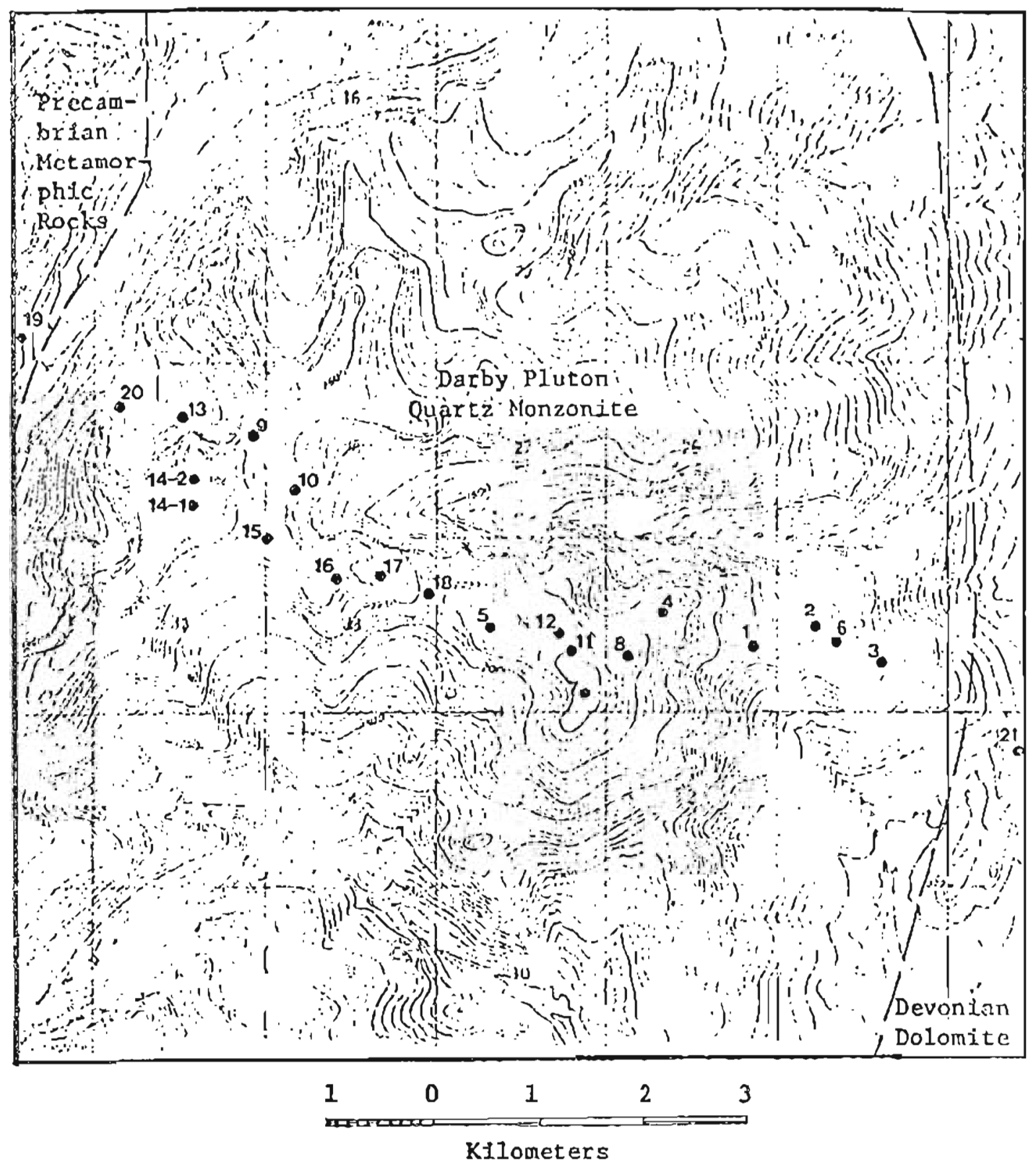

Figure 9. Geologic and sample locality map of the Darby Pluton. - Sample localities. 


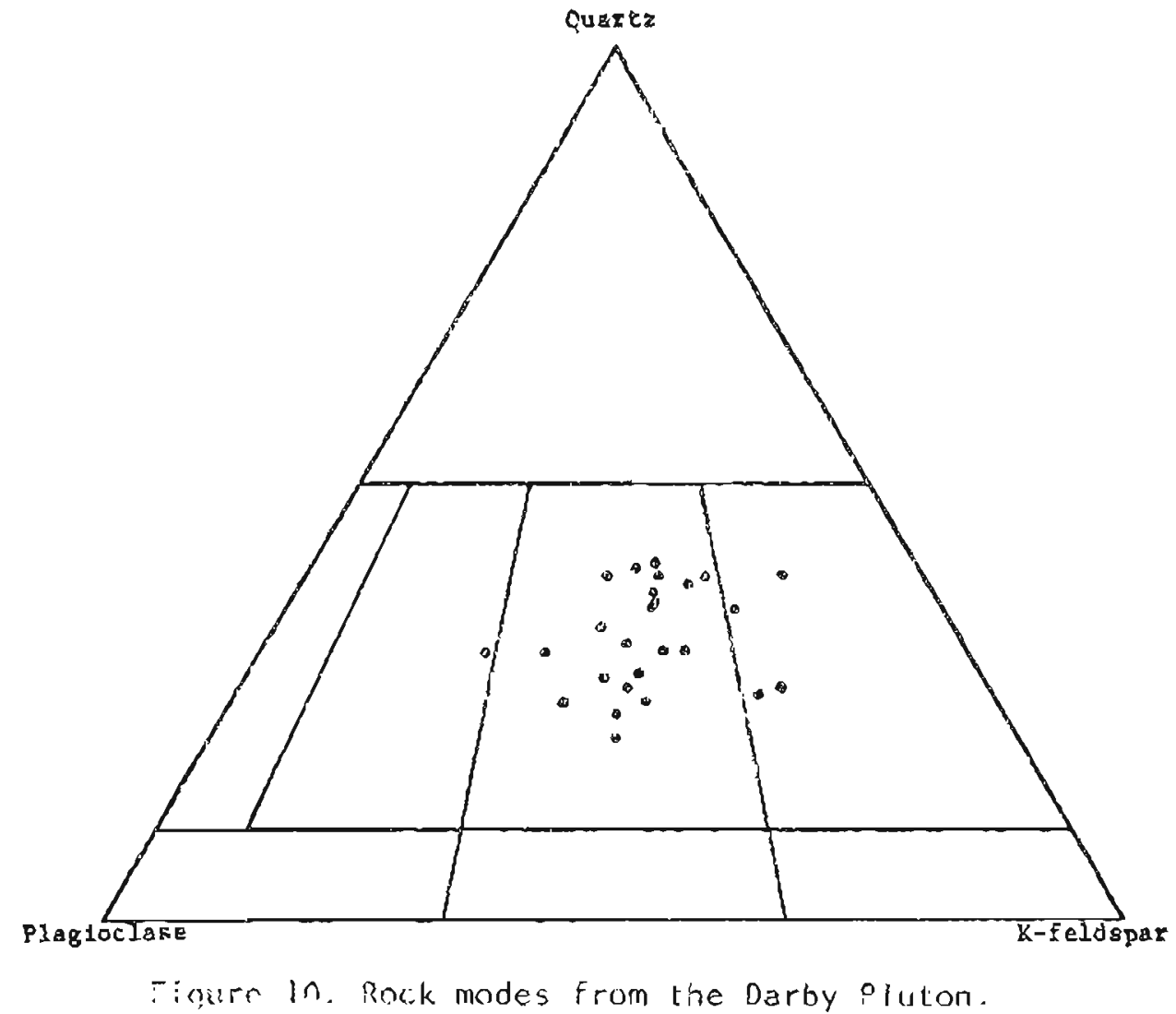

The cuartz monzonite of the Darby Mountains is a coarse-grained hypidiomorphic granular rock showing no tendency towards foliation of mineral grains. Orthoclase and plagloclase occur in approximately equal amo.nnts. K-feidspar crystals are typically the largest phase in the rock, up $: 02 \mathrm{~cm}$ in length. The K-feldspar is perthitic and subhedral. Plagioclase An $18-27$ occurs as interstitial anhedral grains and subhedral crystals showing norma? zoning and some aiteration of the more calcic cores.

Quartz occurs as interstitial grains and occasionally as large crystals of suohedral habit. Biotite is the oniy mafic mineral and consticutes no more than $5 \%$ of the rock. It is partially altered to chlorite in some specimen's.

\section{Potassium Analyses}

Potassium analyses from the rock sanples collected from the Darby Piuton are presented in graphical form in Figure ll. There appears to be no aign!ficas iystematic change in the potassium content across the pluton.

\section{Zane Hiils}

The Zane Hills form a small mounlain range trending N30W, lacated on the Koyukuk River near the east end of the Hogatza Plutonic Be!t. They are cored by the Zane Hills plucon, a Cretaceous batholith consisting of granodiorice, monzonite, hybrid aiorice, ano a previously undescribed unit consisting o? augen gneiss and dike rocks. A geologic and sample locality inap is shown in Figure 12. Modes are shown in Figure 13. 


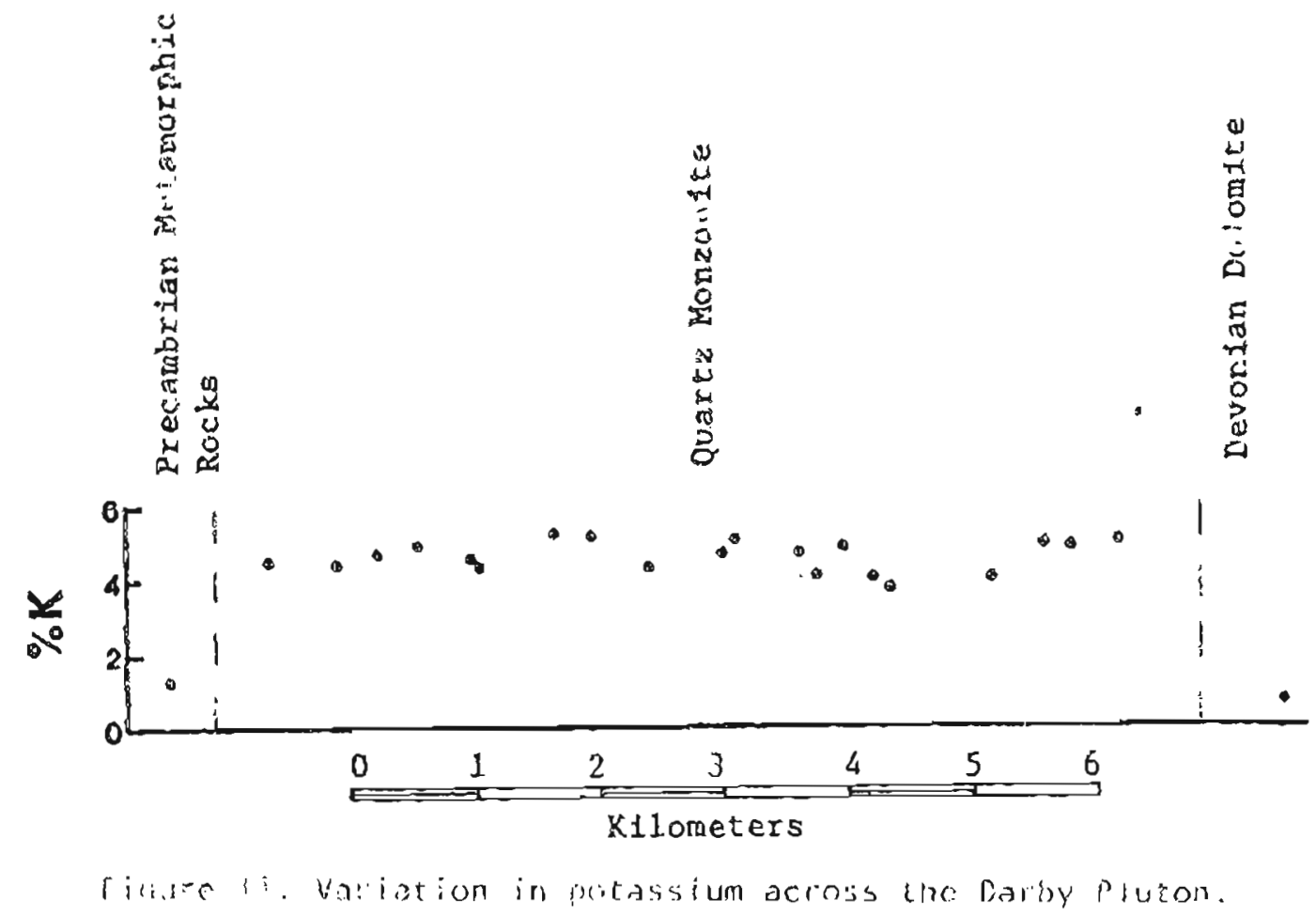

\section{Eranociorize}

The major plutonic phase in the Zane $\mathrm{Hills}$ is an equigranular mediumqrainec granodiorite, dividec by Miller (1970) into a northern hornblende a:anodiorite zone and a soutiern biotite granodiorite zone. Ten samples were collected in a traverse through the hornblende biotite zone.

Plagioclase is the most abundant mineral, occurring in subhedra! grains with oscillatory zoning. Microcline is found as subhedral crystals rimming the plagioclase in a similar cexture to that seen at riranite Moun. iain. Quartz constitutes as much as 20 percent of the rock and is found as anhedral crystals. Bictice is the most abundant mafic mineral, com$2-i \equiv$ inc ayp roxinately $2.5 \%$ of the rock. The biotite is oflen partially $\therefore:=0$ ctorite. Sphene and apatite are the main accessory minerals.

A striking characteristic of the 7ane llills franodiorite is is :exiliral homogeneily.

Auger Grieiss.

Along the east margin of the Zarie 4 ills, an augen greiss zone, approxinateiy I $\mathrm{km}$ wide, was locaced along the north traverse.

Plagioclase occurs as large rounded augens, up to $1 \mathrm{~cm}$ ir. size, in a ine grainec matrix of K-feldspar, plagioc!ase, and quartz. The mafic minerals, hornolende and biotite, are banded and smeared around the large plagioclase crystals in an unmistakable caraclastic texture. Petrographic 


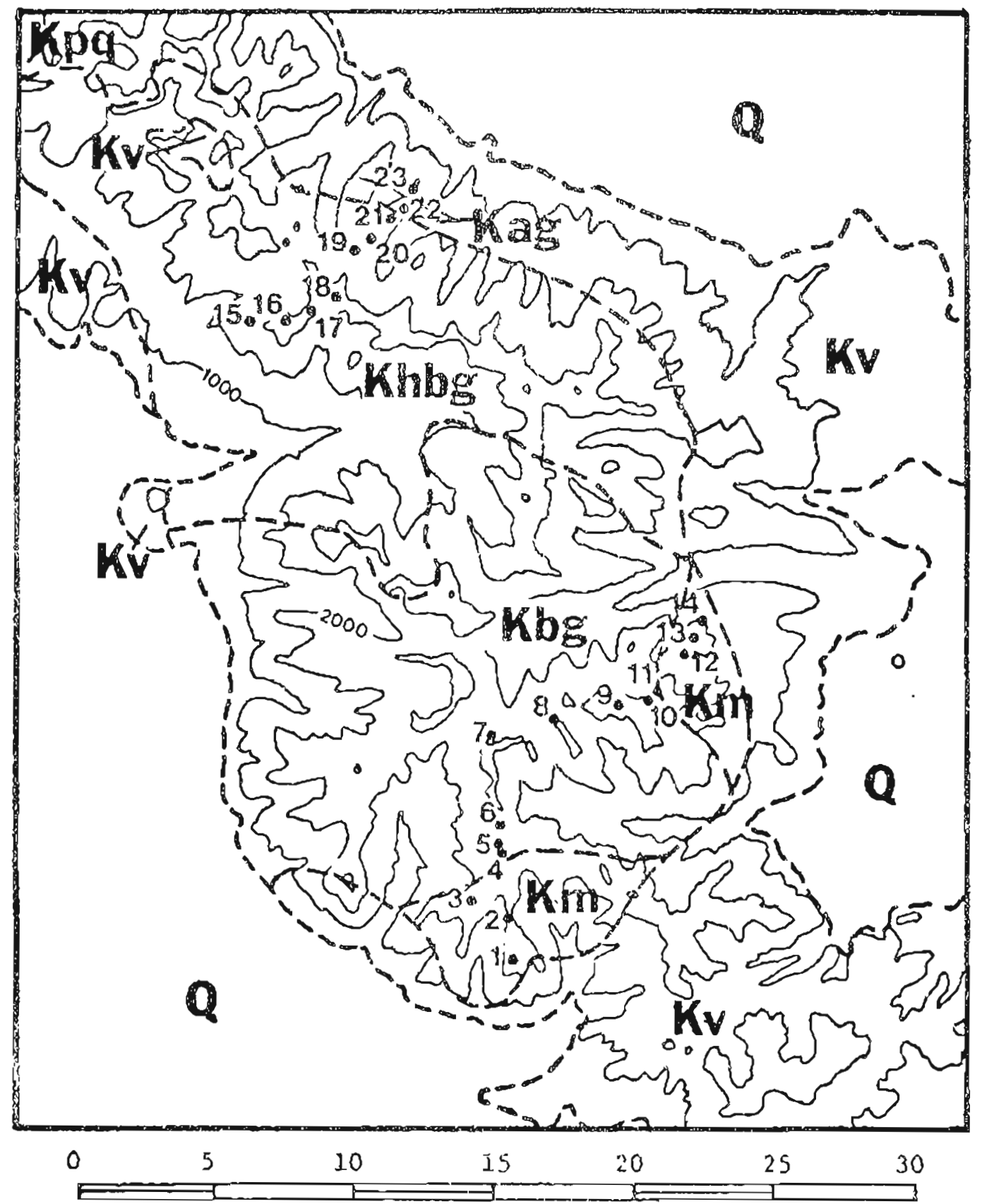

Kjomezers

Explanation

Khbg Hornblende biotite granodiortie Kpq

$\mathrm{Kbg}$ Blotite granodiorite

$\mathrm{Km}$ Monzonite

Kag Augen grelss

Porphyritic quartz morzonite

Kv Volcanic rocks

Q Alluvium

Figure 12. Geologic and gample locality map of the Zane Hills (after Milier, 1970). 


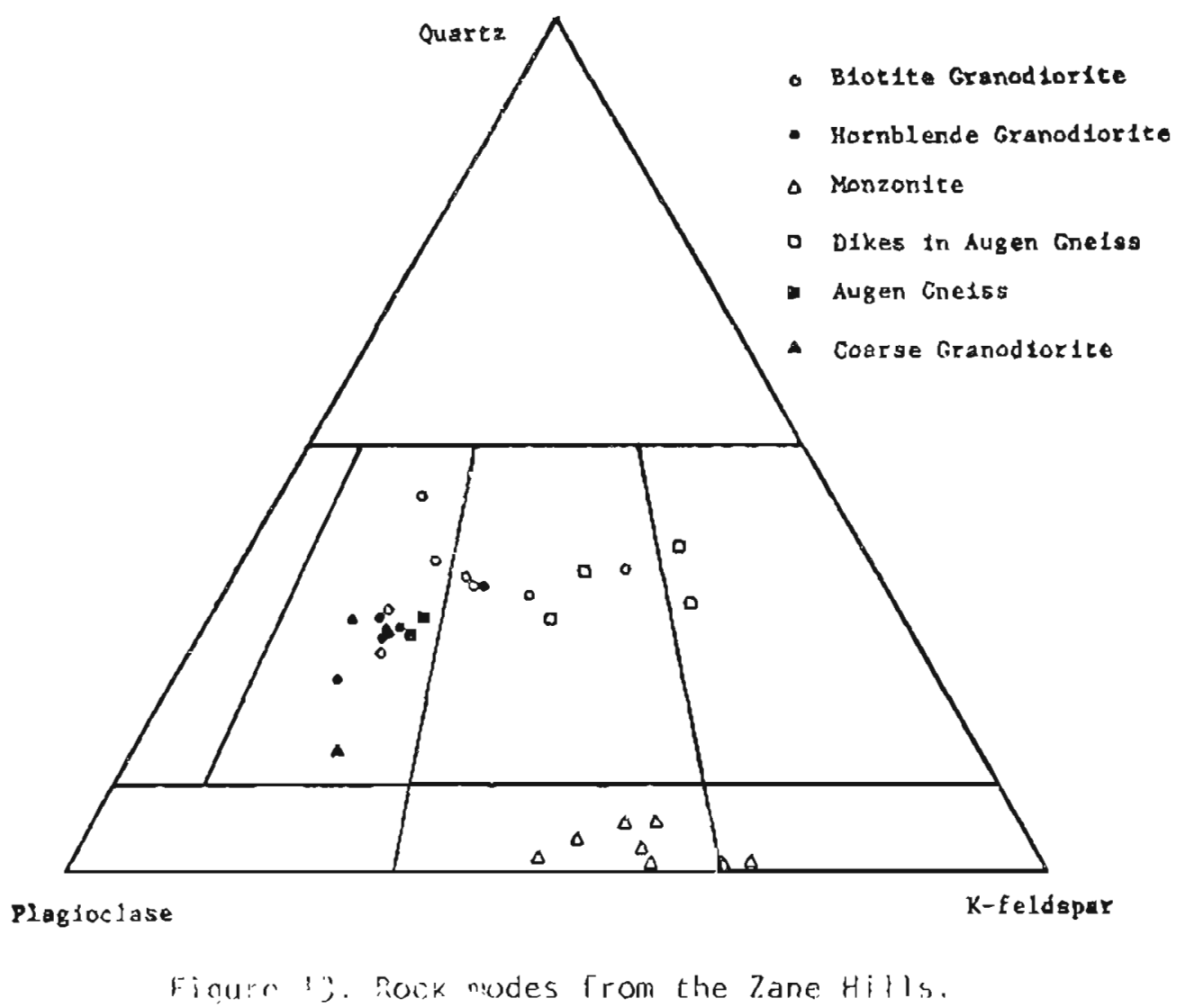

analyses of stained rock slabs and thin sections from these samples show that the mode within this zone is identical 'o the Zane Hills granodiorite (Figure 13). The augen gneiss appears to be a recrystallized phase of the granodiorite, subjected to inten ive shearing. The existence of this phase along the eastern marrin $0^{:}$the 7ane 1 ills pluton suggests a fault contact with the andesilic country rock.

Within the augen gneiss zone other rock types were found, including fine-grained gneissic dikes ano aplite and pegmatite dikes.

Monzonite and Hybrid Diorite

Two zones of monzonite arc iybrid diorite, containing anomalous concentrations of uranium and thor:um are exposed along the southern margin of the Zane Hilis (Figure 12) (M:ller, 1970).

The monzonite is variab!e ' $n$ texture from roughly equigranular to gneissic. Two stages of plagioclase crystallization are apparent. piagioclase An 10-25 occurs in medium to large subhedral grains and as fine-grained late stage myrmekitic intergrowths with quartz. Perthitic microciine occurs in large subhedral grains, up to $3 \mathrm{~cm}$ in size, or interstitially. Interstitial quartz is present in minor amounts. A sea-green amphibole is 
the major mafic mineral, making up as much as 15\% of the rock. Brown biotite is abundant, whereas pyroxene is found only in minor amounts. Accessory mirerals include sphene, apatite, zircon, and rutile.

The hybrid diorite is a contact metamorphic rock. Its origin is the result of the incorporation of xenoliths into the granodiorite magma (Milier, 1970) and the composition of this phase is variable. Hornbiende makes up as much as $70 \%$ of these rocks, often seen rimming pyroxenes. The common cexture is banded and porphyritic with large amphibole and plagioclase crystals in a fine-grained felsic matrix. Plagioclase has altered to sericite in some cases. This rock type contains few accessory minerals.

Miller (1970), in his investigation of the plutonic rocks of WestCentral Alaska, used the term alkaline rock in the sense of Turner and Verhoogen (1960); 'a rock in which the aikali content is sufficiently high a compared to silica for specialiy alkaline minerals such as feldspathoisc : o eppear.' Thus Miller's usage of the term is distinctively Jifferen: from the chemical classification used in this report (see Appendix 2). The Zane Hills monzonite does not contain feldspathoids. As a result Miller does not consider it to be part of the alkaline belt and places the eastward limit of this belt at the Ekick Creek Pluton, $170 \mathrm{~km}$ to the west. A chemical analysis of the monzonite from the Zane Hills is shown in Table 4 .

Table 4

Chemical Analysis of Monzonite from the Zano Hills taken from Miller, (1970).

\begin{tabular}{|c|c|}
\hline Oxides & Wt. \% \\
\hline$\overline{\mathrm{SiO}_{2}}$ & 61.1 \\
\hline $\mathrm{Al}_{2} \mathrm{O}_{3}$ & 12.0 \\
\hline $\mathrm{Fe}_{2} \mathrm{O}_{3}$ & 1.7 \\
\hline $\mathrm{FeO}$ & 1.6 \\
\hline $\operatorname{Mgn}$ & 0.8 \\
\hline $\mathrm{CaO}$ & 2.8 \\
\hline $\mathrm{Na}_{2} \mathrm{O}$ & 5.3 \\
\hline $\mathrm{K}_{2} \mathrm{O}$ & 6.3 \\
\hline $1120^{-}$ & 0.06 \\
\hline $\mathrm{H}_{2} \mathrm{O}+$ & 0.67 \\
\hline $\mathrm{TiO}_{2}$ & 0.39 \\
\hline $\mathrm{P}_{2} \mathrm{O}_{5}$ & 0.16 \\
\hline $\mathrm{MnO}^{\circ}$ & 0.07 \\
\hline $\mathrm{CO}_{2}$ & 0.05 \\
\hline Total & 100.00 \\
\hline
\end{tabular}

Total äkuties $=11.6$, which is greater than $1 / 6 \mathrm{si0}$. According co our classification (after shand, 1922), this is an alkaline rock. The difference in classification schemes extends the belt of alkaline rocks $170 \mathrm{~km}$ east of the margin described by Miller (1972). 
Located on the west side of the Darby Pluton on the eastern Seward Peninsula, the Dry Canyon Creek Stock is described by Miller and others (1972) as a leucocratic to trachytoid foyaite (Figure 14). The stock is cut by blue-gray pulaskite dikes and has been tentatively assigned a midCretaceous age based on a $K$-Ar age of $105 \pm 3 \mathrm{~m} . \mathrm{y}$. (M.A. Lanphere, written communication in Mi!ler and others, 1972).

Modes of $: 7$ rocks determined from examination of stained thin sections and rock slabs are shown in Figure 15. The predominant rock type is a hornblende-biotite nepheline svenite. but other more mafic phases, such as hornblende-aegerine-augite alkali gabbro were also found. One sample of syenite containing less than one percent quartz was found in rubble crop.
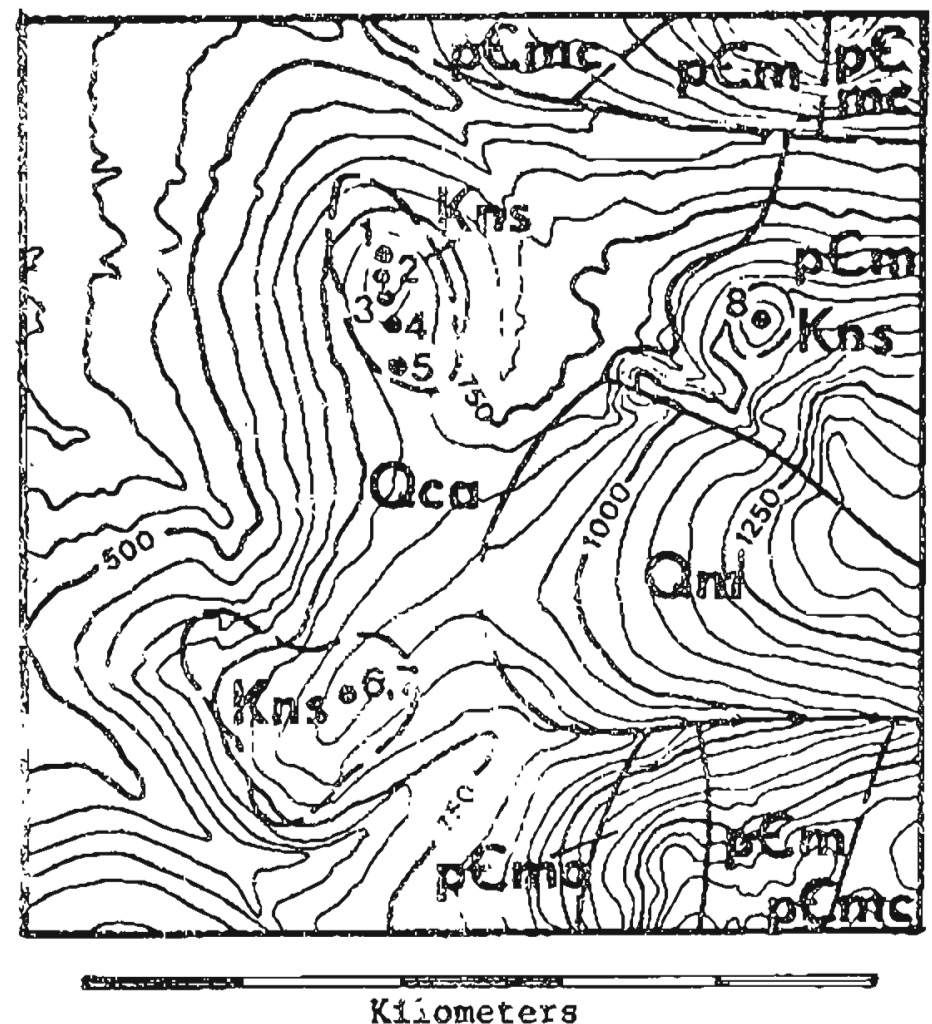

KIiometers

Cretaceous $\begin{array}{lc}\text { Kns- Nepheline Sye- } & \text { Qm--Moralnal Deposit } \\ \text { nite and Alkali } & \text { Qca--Colluvium and } \\ \text { Gabbro. } & \text { Alluvium. }\end{array}$

Precambrian pCme-Metamorph1c Complex pCm--Marble

Figure 1t. renlonic and somnle locality map of the Dry Canyon Creek Stock, as modified from Miller and others (!972). 


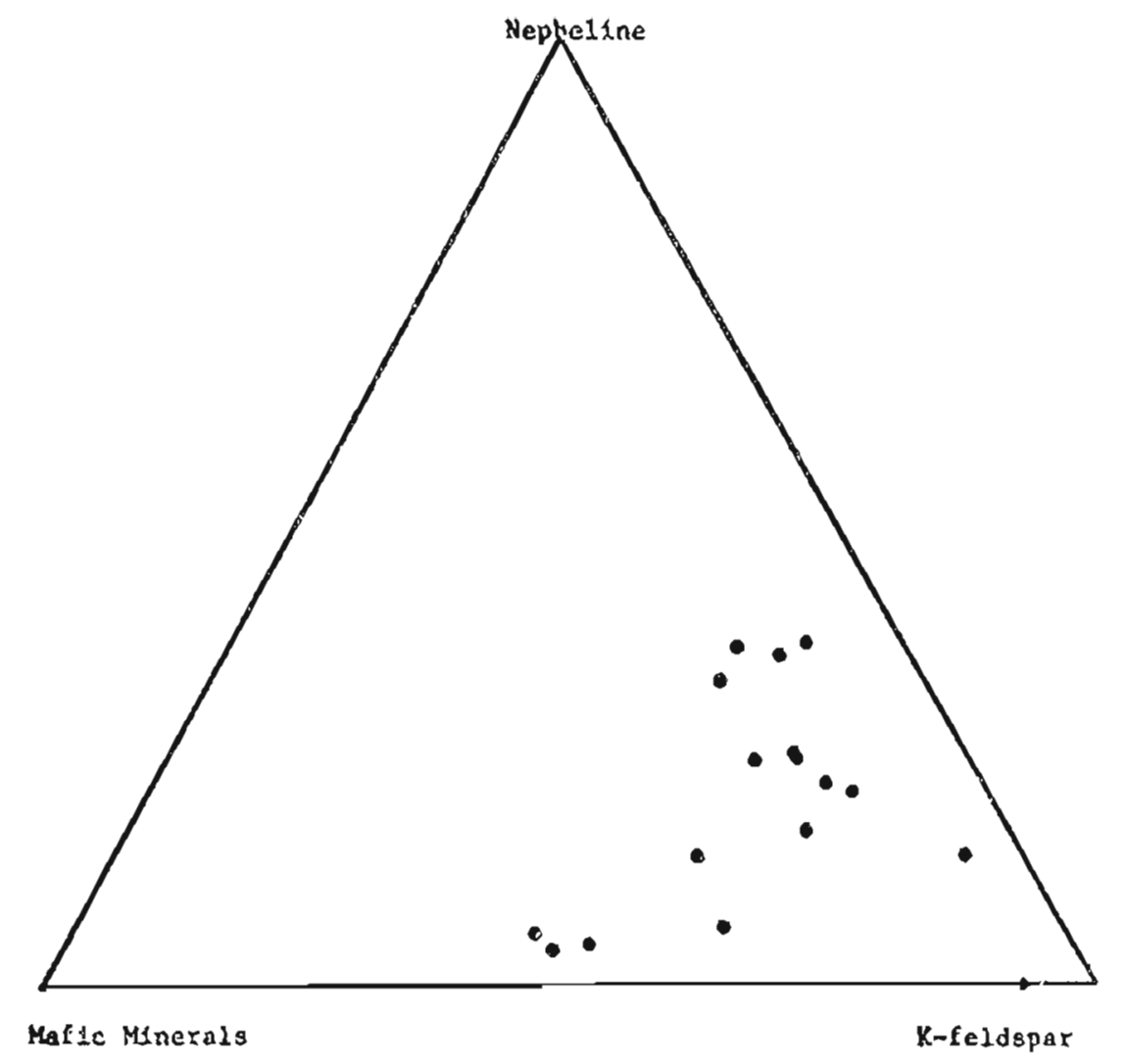

tintre 15. Modea Cí rocil; From lhe Dry Canyon Creek Slock.

The porassium feldspar is anorthoclase which occurs as subhedral grains, of ten showing a mottled scotch plain twinning, distinctively fifferent from that found in microcline. The maximum size of the anor:hoclase crystals is $5 \mathrm{~mm}$. Henheline, which makes up as much as $35 \%$ of the rock, is in interstitial crystals, which have been largely altered to cancrinite. Often the anorcnoclase and nepheline show evidence of exsoiution structures including graphic and myrmekitic intergrowths, and what Bower (1928) described as a fingerprint texture.

Plaqioclase occurs as a minor constituent in some of the samples, and a major rock forming mineral in one case where it makes up $14.7 \%$ of the rock.

Biolite, hornblende, and pyroxene are found in varying proportions. Bintits the major mineral in the leucocratic varieties where its iarit: in gioneroporphyritic or in bands and streaks.

The pyroxene is most common in the alkali gabbros where it is distinctively zoned, showing sodium enrichment towards the rim and a stronger augite component towards the center. The pyroxene makes up as much as $25 \%$ of some of the rocks sampled. 
Brownish hornblende is found in both the leucocratic and mesocratic varieties, sometimes as reaction rims around the pyroxene, and in other cases rimmed by biotite. Hornblende anc biotite are interstitial to the felsic minerals whereas the pyroxenes are subhedral and appear to have formed prior to crystallization of the light colored minerals.

The predominant texture is one that appears to be glomeroporphyritic, but is actually a relict texture rerulting from the exsolution of large subhedral leucite crystals, in a mat ix of mafic minerals, predominanty brotite. The texture appears as cimcular aggregates of nepheline and anorthoulase, surrounded by bands oi biotite and hornblende. Upon careful examination some of the round crnss sections appear to have eight sides, a relict of the original cryitallization history. Flow banding or perhaps a minor shearing of the rack during the latest stages of crystallization have caused some disiortion and elongation of the pseudoleucite cross-secrions. This period of deformation could not have been very severe because little evidence if ataclastic deformation is evident along grain boundaries.

Much of the biotite has experienced severe radiation damage. There are abundant pleochroic haloes which are the result of uranium and thorium in zircon and apatite crystals. The apatite appears to contain a much higher concentration of uranium and thorium than in other rocks examined in this study, as evidenced by the width of the haloes. In one sample, the margins of the biotite crystals are black. These crystal margins looked quite similar to the pleochrour haloes caused by Lhe radioactive acressories incorporated within the biotite. This distortion of the pleochroism along grain margins suggests that the adjacent minerals, in this case hornblende. nepheline and anorthoclase, are high in uranium and thorium.

A lignt colored garnet, perhaps andradite, is present in substancial amounts ( $\dot{H}_{\%}$ ) in some of the samples. The garnet occurs as anhedral grains in roughly circular swarms, suggesting shat a number of these small equant grains are part of a much larger garne: crystal.

Melilite, characterized by high re! ief, low birefringence, a uniaxial negative sign and light yellowisn coior, is seen in three of the samples, making up as much as $5.6 \%$ of the rock The mineral occurs as small interstitial grains, and occasionally as e:ongate subhedral crystals up to $1 \mathrm{~mm}$ in length.

Carbonate is found in all of the samples in minor or accessory amounts. It is difficult to tell whether the carbonate is the result of remobilization of calcite or dolomite from the nearby metamorphic terrain, or if it is primary. The carbonate grains are very small, usually $0.1 \mathrm{~mm}$ in size. They are anhedral but do not appear to be restricted to vein areas, nor do they appear to be replacing other minerals.

The fact that the carbonate minerals are found in so many of the sections as disseminated grains, and the coexistence of melilite in these rocks strongly supports a primary origin. The occurrence of carbonate-bearing vein rocks of primary origin in the Selawik Hills (Eakins and others, in press) a) so supports this conclusion. 
nuartz occurs as an arcessory mineral in one syenite sample. Other accessory minerals include sphene, apatite, and zircon.

\section{Selawik Lake Complex}

The Selawik lake complex is a small ( $18 \mathrm{sq} . \mathrm{km}$ in area) alkaline pluton lacated on the south shore of Selawik Lake in western Alaska. The northern half of the complex is composed of massive leucocratic juvite (Miller, 19\%2). Melanite garnet is abundant, constituting as much as 15 percent of the rock. The southern half of the pluton is poorly exposed and consists of perthosite and malignite. The alkaline stock is in fault contact with a syenite that contains minor amounts of quartz (M: 11er 1972).

Modes of 10 rock samples from the Selawik Lake Complex are shown is Figure 17. A locarion and geologic map is shown in Figure 16. Sample localities are restricted to the notth side of the pluton because outcrops are virtually absert in the southern half. Of particular interest are samples AP-6A, 8 ; and $C$, taken from a zone of dike intrusion and alteration that contains uranium values as high as 32 ppm.

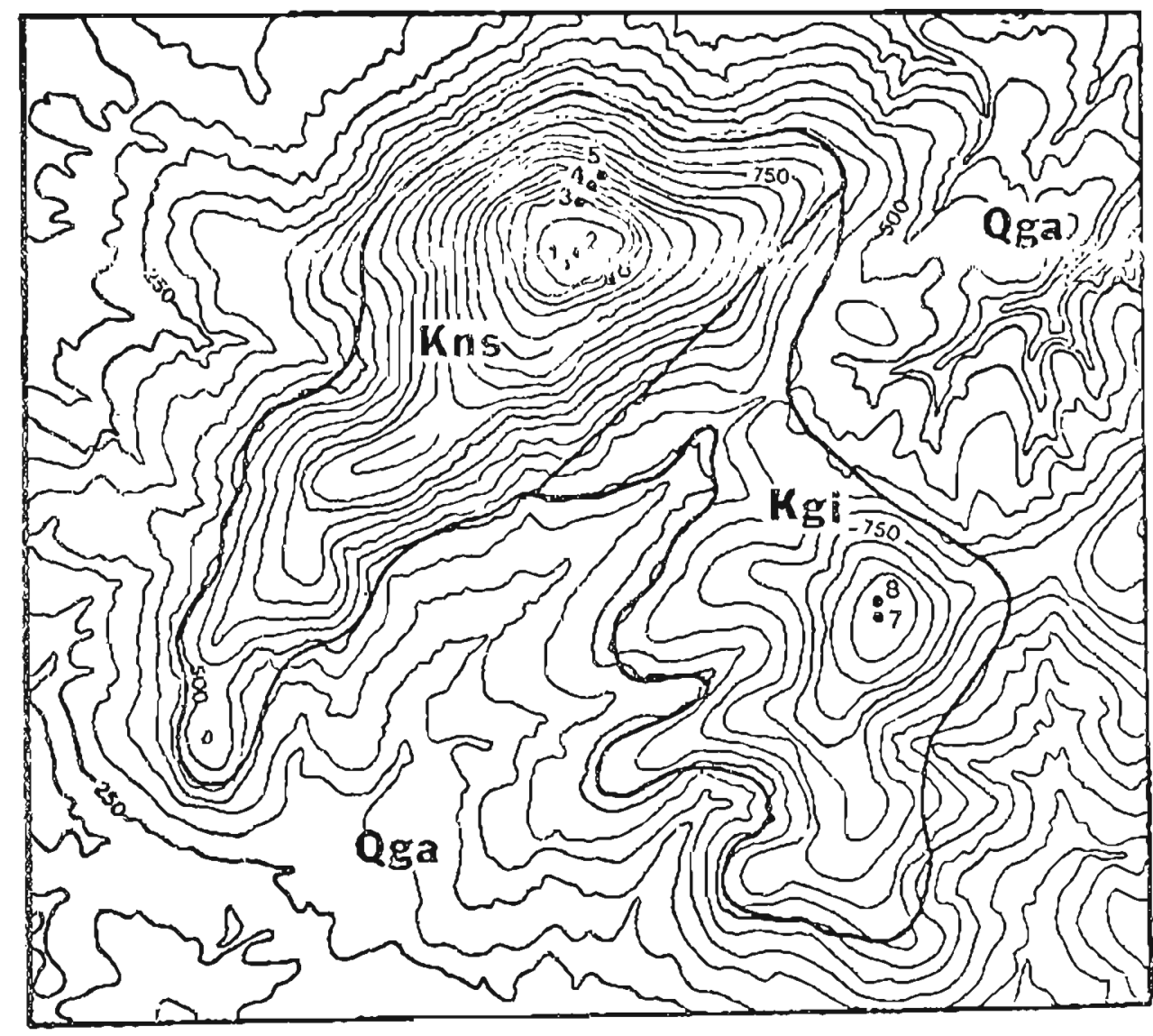

Kllometers

Explanasion

Cretaceous

Kns-Nepheline Syendte

Kgl-Syenlte and Monzonica
Quaternary

Qga-lindfferentlaced Glacial Drift

Figure 16. Location and seologic map of the Selawik Lake Comolex. 


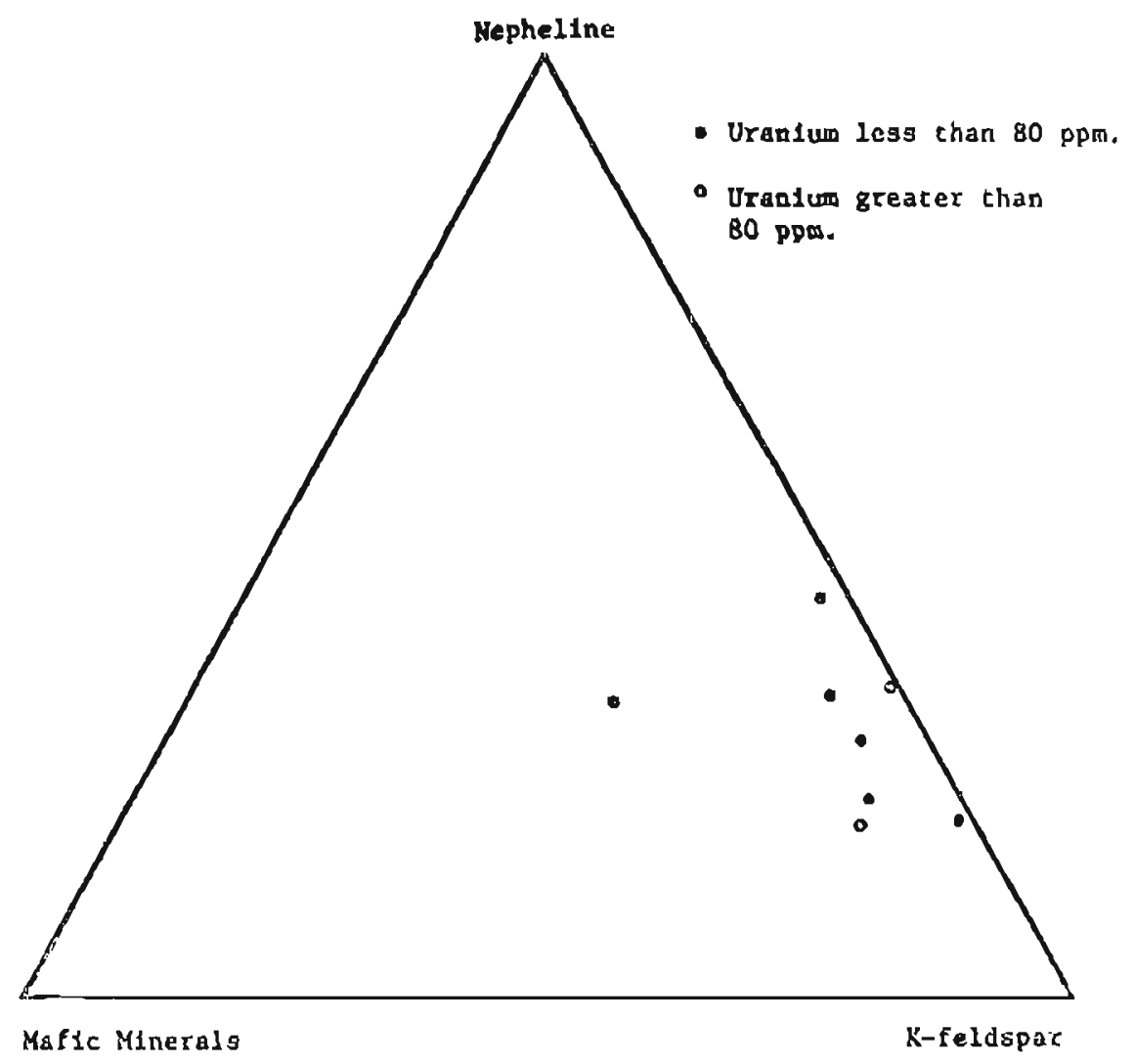

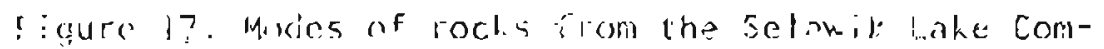
D) ex.

The major rock type found in the samples collected during the 1975 field season is a garnet and aegerine-bearing biotite nepheline syenite. The major minerals inciude orthoclase, nepheline, cancrinite, aegerine, biotite, and melanite garnet. Kalsilite has also been identified (Miller, 1972).

The predominant textures found in these samples are complex intergrowths that are the result of exsolution. A fine-grained fingerprint intergrowth is found in most samples studied and less commonly, a medium-grained graphic exsolution texture. The complexity of the exsolution made the determination of the modes difficult and a large error is associated. Petrographic analysis was aided by the use of a methylene blue nepheline stain (see Appendix 4 ). The stain clearly defined the nepheline in the fingerprint intergrowths. A number of intergrown crystals were first point counted separately at closely spaces intervals to determine the average ratio of nepheline to $\mathrm{K}$-feldspar within the intergrowths. After the percentage was determined the intergrown crystals could then be counted as one phase and the proper proportions of the two felsic minerals determined after counting was completed.

The habit of the nepheline and k-feldspar crystals seen in these rocks is the result of these exsolution textures. K-feldspar is orthoclase, which sometimes occurs as very large crysials, up to $10 \mathrm{~cm}$ in size. In hand specimen 
The foldspar is often ro'se colored. Wepheline is antedral, in smaller yrain: thai are sometimes altered to cancrinize. Placiaclase was not found in any of the sections studied.

Mafic minerals are commonly found in glomeroporphyritic crystals and in bands. Biotite, the most common mafic mineral, is a dark brown variely ofien showing distinct pleochroic haloe's, as the result of radiation damage from inciuded apatite and zircon crystals. The pyroxene $i$ a deep green aegerine, somelimes rimmed by biotite, in subhedral grains approximately $1 \mathrm{~mm}$ in size. Melanite garnet is intimately associated with the pyroxene in some sections, suggesting a genetic relationship. It appears that the garnet has exsolved from the pyroxene. This could occur if an aluminous pyroxene had crystallized initially at considerable depth and then had exsolved the Altt ion as the result of a decrease in pressure. The aluminum could then go into the formation of narnet, a melanite form of andradite if enough $\mathrm{Ti}^{l_{4}+}$ was present. Sphene is also found in some of the glomeroporphyritic crystals; suggesting that titanium was abundant.

A dark reddish-brown ampnibole occurs as a minor mineral. The accessory minerals include apatite, zircon, fluorite, and pyrochlore, a thorium-bearing mineral associated with nephe? ine syonile.

Tist: rock samples from the adiacent syenite wert analysed for their urar. "un an thoriun content and their modes determined by petrographic m:iy, $;$. The syenite is mediunt-grained, with a gneissic texture, and coniains iks than lis quartz. Whereas olagioclase is absont in the alkaline stock to lhe west. in the syenite unit it makes up 6-83\% of the rock.

Aegerine-augite and an amphibole are the mafic minerals, making up as much as 15:x, of the rock. The amphibole has a striking blue-green component to ils pleochroic scheme, suggesting a high $\mathrm{Na}$ concentration.

As noted in the discussion of the nonzonice at franite Mountain, and in the monzonite in the Zane fiills, the classification as alkaline by Shand (1922) would probably include this rock type. Although quartz is present in ininor ariounts, the presence of quartz is probably the result of abundant cal.cium, magnesium, and iron, which al lowed the early formation of sodium-bearing amphiboles and pyroxenes. The incorporation of sodium in these minerals depleted the melt in this component during later stages of crystallization. As a result, nepheline did nos: form, and the percentage of $\mathrm{SiO}_{2}$ in the rock was sufficient to allow the crystallization of quartz. Although there is a disfinction between the syenite and the nephei ine syenite in terms of their bulk chemistry, tis difference is enhanced mineralurically because of the abundance of $i$ on. caicium, and magnesium in the syenite which removed the aikalies from the moil zrod is not allow the formation of nepheline as a late state crystaliizarion product.

This is an interesting conclusion because it suggests an additional model for the formation of complexes that contain both alkaline and calc-alkaline rocks. Perhaps the mechanism for the formation of zoned intrusives is based on the rractionation and distribution of the mafic elements, which in turn control the crystallization and distribution of the alkali elements. 


\section{GEDCHEMISTRY OF URANIUM AND THORIUM}

\section{Igneous Rocks}

During most of the magmatic cycle, uranium arid thorium are in the tetravalent state and the crystallization paths of the two elements are quite similar because of their similar ionic rad;i. The ionic radius of uranium is approximately $0.97 \mathrm{~A}$ in sixfold coordination and $1.01 \mathrm{~A}$ in eightfold coordination; that of thorium is $1.02 \mathrm{~A}$ in 6 fold, and $1.06 \mathrm{~A}$ in eightfold coordination. Plus 4 is the only stable valence state of the thorium atom, whereas uranium, under oxidizing conditions, readily changes from +4 to a +6 valence state decreasing its ionic radius to $.8 \mathrm{~A}$. This property causes uranium and thorium to follow strikingly oifferent paths during the latest stages of crystallization, and during the weathering cycle.

During crystallization, when the water content of the meit is very iow, part of the $\mathrm{U}^{4+}$ and $\mathrm{Th}^{4+}$ present enter host minerals, proxying for $\mathrm{Zr}$ in zircon, and $\mathrm{Ca}$ in apatite and sphene. Coordination requirements prevent uranium and thorium from substituting for calcium in plagioclase.

Almost all compounds of $\mathrm{U}^{4+}$ and Th4t are known to be highly insoluble in aqueous solutions in the laboratory. Late in the differentiation of a magma, water may so reduce the solubility of both uranium and thorium that sporadic precipitation of actual uranium and thorium minerals may occur (Larsen and Phair, 1954).

At a very late magmatic stage, uranium is commonly oxidized to the $\mathrm{U}^{+6}$ valence state, and forms a number of soluble compounds particularly with $\mathrm{CO}_{\bar{z}}$ and $\mathrm{SO}_{\overline{4}}$ anions. Thorium has only one stable valence state and it is not affected by the shift to oxidizing conditions.

Uranium is associated with alkaline igneous rocks, and particularly with the aspaitic or peralkaline group of alkaline rocks. Such rocks contain more total alkalies than aluminum. Although the rocks examined in this report are highiy potassic, because of their high aluminum content none of them would fall in the agpaitic or peralkaline cacegory of alkaline rocks.

On the whole uranium concentrations in volcanic rocks appear to be slightly higher than concentrations in their plutonic equivalents. In addition to uranium concentrated in accessorv minerals, the glass phase often is enriched (Adams, 1954). In the glass phase uranium is easily ieached whereas only a small percentage. of the uranium in the common accessories can be mobilized during weathering processes.

\section{Sedimentary Rocks}

By far the greatest quantity of uranium is found in sandstone-uraniumvanadium-copper deposits (Stanton, 1972). The greatest development of these ore bodies occurs in the western and southwestern United States where they are be! ievec to have formed in an arid or semi-arid environment. 
The usual host ruoks of these "sandstone" deposils are conglomerates, sendstone, and silistonas dithin bilase rocks, the deposits are related co a variety of sedimentary features: contacts of coarse sediment with mudstone, thickness of the coarje unit, paleostrean channels, and plant remains.

It is generally agraed that these deposits ore epigenetic, but the source of the uraniun-bearing fiuios is unknown. The uranium deposits are the resul? of the reduction of uranium compounds, such $a \mathrm{~S}^{\mathrm{V}}\left(\mathrm{SO}_{4}\right)_{3}$ and $\mathrm{U}\left(\mathrm{CO}_{2}\right)_{3}$. The denocis, acur in uranium rolls, at the boundary between oxidized and re

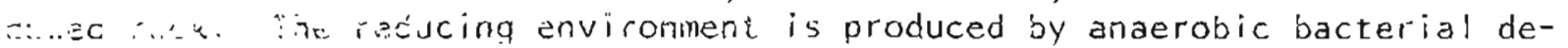
Lav ano ine forination of 'rizs from the breakdown of plant remains.

Thorium is not a major constituent in these deposits. It is not easily monilized sy the wezthering processes, and i's not transported by oxidizing teictherma soìtinns.

\section{Sea Water and Ocean Basins}

Uranium wili conbine in sea water with sulfale and carbonate ions to form soluble compounds. As in the sedimensary deposits, it requires reduction in order for precipitation to occur. Such an environment is possible in peat bogs and $i n$ iagoons and closed basins where circulation of the waier does not occur.

in Alaska, where peat bog: are abundant and where sizeaislo fluctuation: in seo level have occurred, particularly during Pieinlocene line, offshore su: merged peat deposits and associated uranium concentrations deserve consideralion.

Thorium would cend to concentrate in placer deposits offshore because it i. la": baning dus ing weathering processes in heavy accessory minerals.

$$
\text { CISTUSSION OF URANIUM AND THORIUM DAIA }
$$

\section{introduction}

One hunored taighi rock samples were anaiysed for uranium, thorium: and ootasilum during the course of this stucy. The results of the analysis pilus abu idied mineralogical data are located in Appendix 1. A discussion of the meshods usec for andiysis is given in Appendix 4.

Average vaiues and standar deviations for the analytical data are giver is Tabie 5. Hislograns of the relative abundances of uranium and thorium are shown in Figure 18. With the exceplion of a few extreme values, the uranium disiribution closely approximates a siraight ine when plucted on log probabiyity paper, reflecting a log normai distribution (Figure 19).

$$
\text { Table } 5
$$

Mearis, Standard Deviations. Maxima, and Minima for Uranium

\begin{tabular}{|c|c|c|c|c|}
\hline sutun & \multicolumn{4}{|c|}{ Uraniuni } \\
\hline & lean & SLd. Dev. & Maximum & Min imun \\
\hline Er-anite Mountain & $\overline{2.9}$ & 1.6 & 6.8 & 0.8 \\
\hline Därby & 5.9 & 3.4 & 14.5 & 1.3 \\
\hline Zane Hills & 5.2 & 0.1 & 49.0 & 0.3 \\
\hline
\end{tabular}
ard Fhor iuri Data For Five Plutuns in Western Alaska. 
Table 5 (Cont.)

\begin{tabular}{|c|c|c|c|c|}
\hline \multirow[t]{2}{*}{ Piuton } & \multicolumn{4}{|c|}{ Urarium } \\
\hline & Mean & Std. Dev. & Maximum & Minimum \\
\hline Ory Canyon & 7.3 & 3.9 & 20.3 & 3.9 \\
\hline Selawik Lake & 12.9 & 25.8 & $92 . c$ & 1.0 \\
\hline Total & 5.8 & 9.6 & 92.0 & 0.3 \\
\hline
\end{tabular}

\begin{tabular}{|c|c|c|c|c|c|}
\hline \multirow[t]{2}{*}{ Pluton } & \multicolumn{4}{|c|}{ Thorium } & \multirow[t]{2}{*}{ Observa } \\
\hline & Mean & std. Dev. & Maximur & Minimum & \\
\hline Granite Mountain & 32.8 & 17.6 & 67.7 & 8.9 & 28 \\
\hline Darby & 37.1 & 15.3 & 64.3 & 13.5 & 22 \\
\hline Zane Hills & 25.4 & 20.1 & 74.0 & 3.1 & 32 \\
\hline Dry Canyon & 55.9 & 14.8 & 76.0 & 31.5 & 16 \\
\hline Selawik Lake & 34.2 & 18.9 & 70.3 & 8.3 & 10 \\
\hline Total & 35.0 & 19.9 & 76.0 & 3.1 & 108 \\
\hline
\end{tabular}

Major Uranium Anomalies

As shown in Figure 18, seven samples lic distinctively above the rustering of uranium concentrations. These anomalies are briefly disussed below.

Selawik Lake Complex

Uranium values as high as 36 and 92 ppm were detected from an area of dike intrusion along the east side of the Selawik Lake Complex (samples $A P-b a$ and $c$, Figure 16). The dike strikes approximately east-west, and $i$, composed of $71 \% \mathrm{~K}$-feldspar, $18.6 \%$ nepheline, $8.6 \%$ biotite, $0.6 \%$ pyroxene, and $1.8 \%$ accessories. The accessory minerals include fluorite, pyrochlore, sphene, ano apatite, and at least one other minor mineral that could not be identificd using $x$-ray techniques. High concentrations of uraniun are not restricted to the dike phase alone. One sample of the adjacent pluton containeó 92 ppm uranium.

\section{Tane Hills Augen rineiss}

Within the area of the Zane Hills augen gneiss unit, two pegmatite dikes were found to contain uranium concentrations up to 49. ppm. The highest value was determined from a penmatils having the composition of $a$ juarty monzonite. The augen gneiss zone is characterized by abundant vining, sno additional aplitic vein rocks were analysed. These, however, did not show high concentrations of uranium. The fact that the pegmatite and aplite dikes are in large part restricced to the augen gneiss unit suggests that they are genetically interreiated to the augen gneiss or that the zoris is an area of structural weakness and that the d kes $f: 11$ Fractures within tne unit.

Dry Canyon Creek Stock

A sample of hornblende-biatite-nephei ine syenite, containing abundant accessory ninerals was found to contain $20.3 \mathrm{ppm}$ uranium in a sample from the Dry Canyon r reek Stock. 


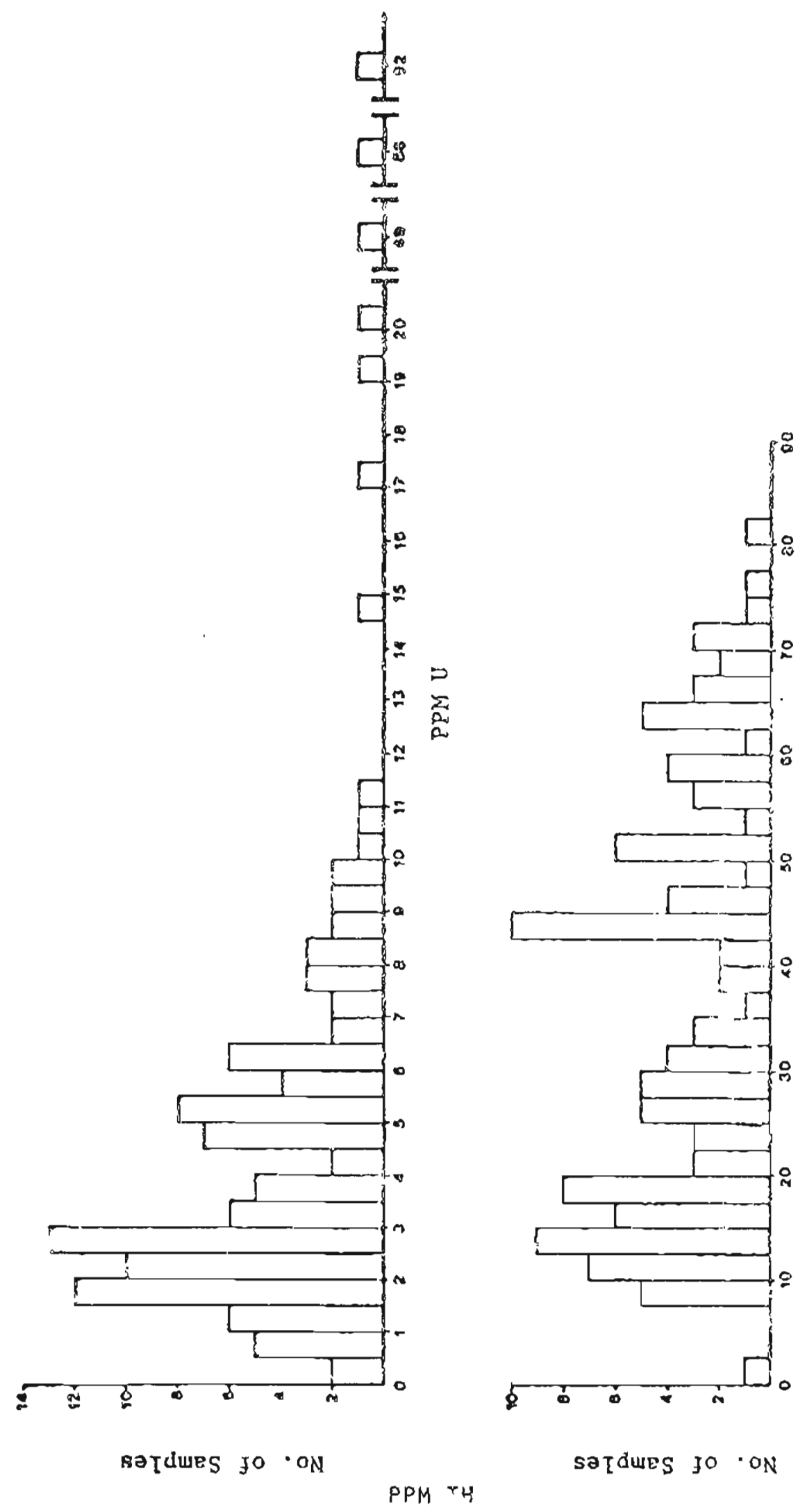

Figure 19. Relative abundance of uranium and thorium within the study area. 


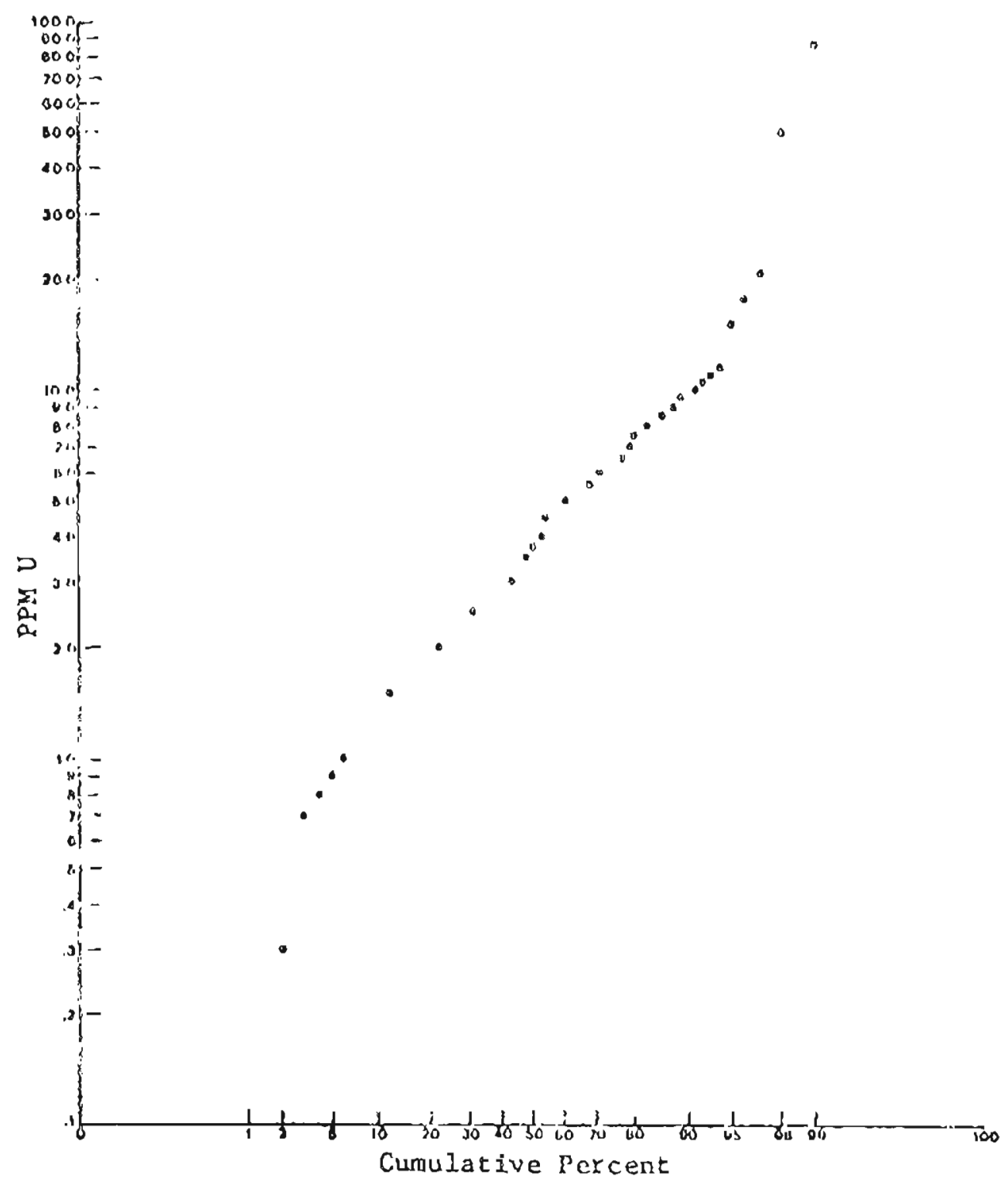

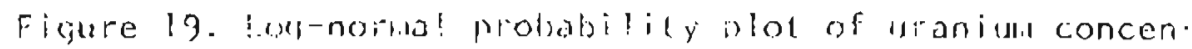
reations.

\section{Darby Mounlains}

A ur.nium concentration of 14.5 ppm was detected in a quartz monzonite - ... : is darty Mountains. Although this vaive is high in terms of this wy "viiier and Bunker (1975a) have analysed a number of samples from the misy Mouncairs snowing similar conceatrations.

\section{Asjessment of Uranium Anomalies}

The above areas, particularly the locations in the lane flills and the Selaw:k Lake Complex are of economic interest and serve as good target areas for future exploration. Both areas drain into basins that may confain Tertiary 
sediments beneath the tundra cover. Recent uranium analyses from the monzonite unic in the Zane Hills by Statz and Miller (1976) have yielded uranium concentrations as high as $130 \mathrm{ppm}$.

A uranium concenträtion of 100 ppm is not uncommon in alkaline rocks in the rest of the world. In the 111 imaussaq intrusive, at freenland, the average uranium concentration is greater than $200 \mathrm{ppm}$. In Alaska the Bokan Mountain alkali granite contains a range of 20-200 ppm uranium disseminated throughout the pluton.

The price of $U_{3} \mathrm{O}_{3}$ is negotiable and as a result is highly variable. Today's average cost is about $\$ 20.00 / 1 b$. Price is dependent on the location of the mine. and the nature of the deposit. The current minimum ore grade is 0.' ian although one mine in West Africa, where labor costs are exceecing: $y^{-}$: Jw, consists of $a 0.05 \%$ deposit, in pegmatites.

Because of the high transportation costs, the minimum current ore grade in Alaska is about $0.2 \% \mathrm{U}_{3} 08$. Although $100 \mathrm{ppm}$ is far from ore grade, the abundance of plutons containing leachable uranium in the Selawik Basin and Seward Peninsula, provide ample source areas for secondary Colorado-type uranium depusits in the adjacent Tertiary Basins. In addition, with the rapid growth in the cost of uranium, the United States will soon begin expioiting some of the lower grade deposits.

\section{Uranium Analyses of Mineral Separates}

Individual minerals were separated from five rock samples and analysed for their uranium concentrations. The results of these analyses are presented in Table 6. Uranium is highly concentrated in sphene, apatite, and allanite. The highest concentration was detected in a small sample that appeared to contain a few minute crystals of uranothorite.

Table 6

Lianium Eoncentrations in Mineral Separates and Estimation of the : ontrabucion of individual Mineral Phases to the Whole Rock Uranium Content

Granite Mountain

$$
\text { U-ppm } \times \text { \% }
$$

Minerai

Quartz +

fe!dsoar

Amphibole

Pyroxene

Biotite

Sphene

Apatite

Allanite

Urano.

thorite'

$\begin{array}{ll}-3 & 90.5\end{array}$

$?$

$-3 \quad 2.5$

$-3 \quad 5.6$

$-$

234

$612 \%$

$-$

$?$

$?$

1.0

$-$
Zane Hills

$\mathrm{U}-\rho p m \times \%$

i-ppm. of rock of rock U-ppm \% of rock of rock

12.

86.4

10.32

7.

7.5

46.

0.6

0.28

- 23.

$2.34 \quad 1520 \%$

1.1

0.25

$2.45 \% 1146 \%$

1.4

21. 28 :

0.4

$4.58 \%$

\begin{tabular}{l}
$-\quad 1780$. \\
\hline
\end{tabular}

0.1

1.78 
Table 6 (Cont.)

Granite Mountain

$\begin{array}{lll}\text { Whole rock analysis } & 1.2 & 17.2 \mathrm{ppm} \\ \text { Total due to rock forming minerals } & ? & 11.4 \mathrm{ppm} \\ \text { Total due to accessory minerals } & 4.8 \mathrm{ppm} * & 27.6 \mathrm{ppm}^{*}\end{array}$

Darby Mountains

\begin{tabular}{|c|c|c|c|}
\hline Mineral & $U-\rho p m$ & $\%$ of rock & $\begin{array}{l}U \text {-ppm } \times \% \\
\text { of rock }\end{array}$ \\
\hline $\begin{array}{l}\text { Quartz }+ \\
\text { feldspar }\end{array}$ & -3 & 97.6 & $?$ \\
\hline Amphi bole & - & - & - \\
\hline Pyroxene & - & - & - \\
\hline Biotite & 29. & 2.1 & 0.61 \\
\hline Sphene & 1512 & 0.1 & 1.51 \\
\hline Apatite & $646 . *$ & 0.1 & $0.65 *$ \\
\hline Allanite & 948. & $0 . i$ & 0.95 \\
\hline $\begin{array}{l}\text { Urano- } \\
\text { thoritel }\end{array}$ & - & - & - \\
\hline
\end{tabular}

Darby Mountains

Whole rock analysis

Total due to rock forming minerals

rotal due to accessory minerals
$2.8 \mathrm{ppm}$

$.6 \mathrm{ppm}$

$3.1 \mathrm{ppm}:$

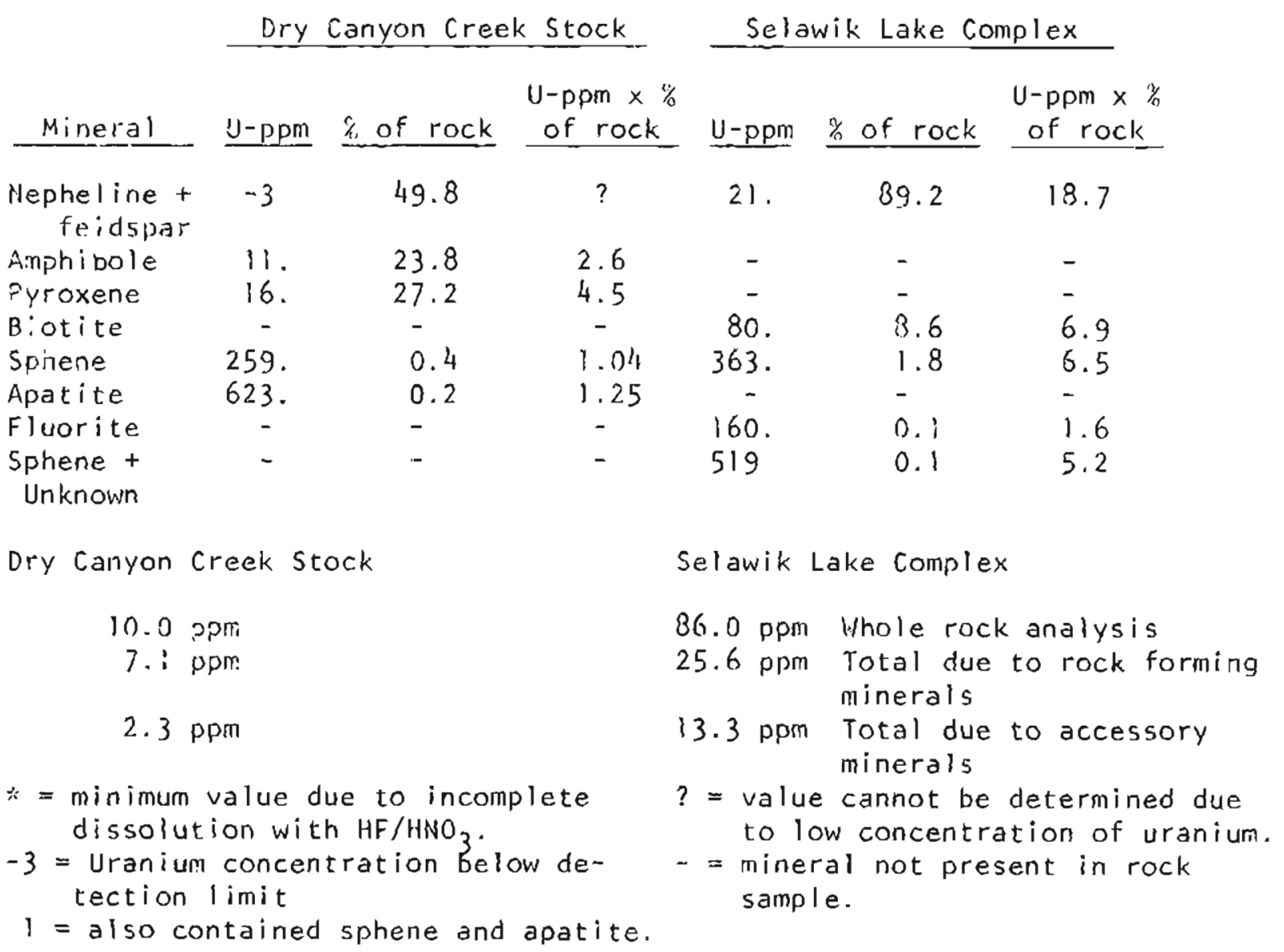


An attempt was made to determine the contribution of each of the mineral phases to the total concentrotion of uranium in the whole rock sample. The percentage of the mineral was multiplied by the concentration of uranium in that mineral. The results of this calculation do not always closely approxinate the uranium concentration as determineo from the analysis of the entire sample.

There are a number of errors that must be considered. Contamination of the rock sample during mineral separation is a strong possibilicy although every precaution was taken in the laboratory to produce clean separates. The samples, particularly those containing the accessory minerals, were often very small which reduces the accuracy of the analysis. The point countins technique used in this study is good to approximately $4.2 .0 \%$ (see Appendix 4). Some of the concentrations of the minerals fall below this limit.

The possibility of an error in the detected uranium value of a highly uraniferous accessory mineral is much less than the possibility of an error in the uranium concentration of the felsic minerals. The inclusion of 1 grain of sphene for example, to every 100 grains of feldspar, would cause a tremendous error in the calculated contribution of the felsic minerals to the concentration in the whoie sample.

One or two grains of quartz or feldspar in a sphene or apatite sample would have little effect on the analysed concentration of these minerals. If we have some confidenca in the uranium concentrations determined in the accessory minerals we can draw an interesting conclusion. Assuming that the percentage of these minerals in the individual rock samples is approximately correct as determined by point count analysis, we can account for all of the uranium in the samples from Granite Mouncain, the Zane Hilis, and the Darby Mountains, by the accessory minerais alone. Howsver, in the two nepheline-bearing rocks, if we make the same assumption we can only account for a fraction of the total uranium concentration from the accessory minerals.

Our conclusion is that either the uranium is concentrated in the felsic phases, in the latter two samples, or there are additional phases that make up very minor proportions of the rock that contain very high concentrations of uranium and are partly included in the rock-forming minerals.

\section{Corralations: U-Th-K-Mineralogy-CPS}

A step-wise multiple linear regression analys ls was performed on the mineralogical and chemical data for the 108 rock samples in this study. The objective of this onalysis was to see if there is some systematic variation between the uranium and thorium concentrations, and the mineralogy of the samples. The Honeywell progran called SMLRP, was used. The program relates a number of independent variabies, in this case, potassium, auartz, plagioclase, K-Feldspar, amphibole, pyroxene, biotite, nepheline, garnet, accessory minerals, and counts per second (CPS from a scintillameter), to e ither uranium or thorium as the dependent variable. 
In addition to performing the step-wise regression on the entire data set, the program was also performed on individual plutons. This analysis nrcvides exce? lent mineralogical guides for the prospector, within individual piutons, and illustrates the wide variety of rock types that contain anomalous concentrations of uranium and thorium.

Analys is of Total Sample Set

The correlation matrix for all of the samples analysed is shown in Figure 20. A correlation of 1.000 represents a perfect correlation, i.e., all of the variance of one variable can be explained by the variance of another. A correlation of 0.000 represents no linear relationship between the variables. Graphical examples of correlation coefficients are shown in Appendix 3. If a relationship between uranium and one of the variables existed and was of the form of a higher order polynomial equation, the correlation coefficient would not necessarily reflect this relationship. Based on the correlation coefficients, the regression builds a linear equation that explains how $Y$ (uranium or thorium) is related to $X$ (mineraiagy or potassium). What we wish to determine is: How much of the variability of $Y$ can we explain in terms of a number of independent variables, $x_{1}, x_{2}, x_{3}, \ldots x_{12}$.

in Figure 20 columns one and two are of the greatest interest economically. Uranium does not correlate well with any of the minerals in the study area. The highest correlation coefficient is a negative relationship with plagioclase, but a coefficient of -0.283 , although significant with this number of samples at the $90 \%$ confidence level, shows only a weak relationship. In terms of the regression equation, plagioclase is the first variable considered: $R^{2}$ is 0.0803 .1 The addition of four other variables, thorium, K-feldspar, quartz, and total accessories only increases the $R^{2}$ value to 0.1468 . We can say that there is very $i$ ittle correlation between any of the mineralogical constituents measjred and the uranium content of the rock, and that we can explain very littie of the variability of uranium in terms of the mineralogical data. This is not a surprising result. As noted earlier, a wide variety of rocks in the thesis area are known to contain anomalous concentrations of radioactive elements. The regression reflects this observation.

The correlations between thorium and mineralogy are only slightly better. After five variables have been added to the regression, we are only able to describe $30 \%$ of the variance of th in terms of the other variables. The wide range in rock types in which high concentrations of radioactive elements are found throughout the thesis area is reflected ay the regression equation for both uranium and thorium.

On a regional scale, in terms of the data available, the uranium and thorium concentrations cannot be precicted in terms of mineralogy. We cannot assist the prospecting geologist by suggesting rock types in

$T R^{2}=$ goodness to fit of the linear equation

$$
=\frac{\text { sum of squares due to regression }}{\text { total sum of squares }}, \begin{aligned}
& =\text { pe } \\
& 0=\text { no tit fit }
\end{aligned}
$$




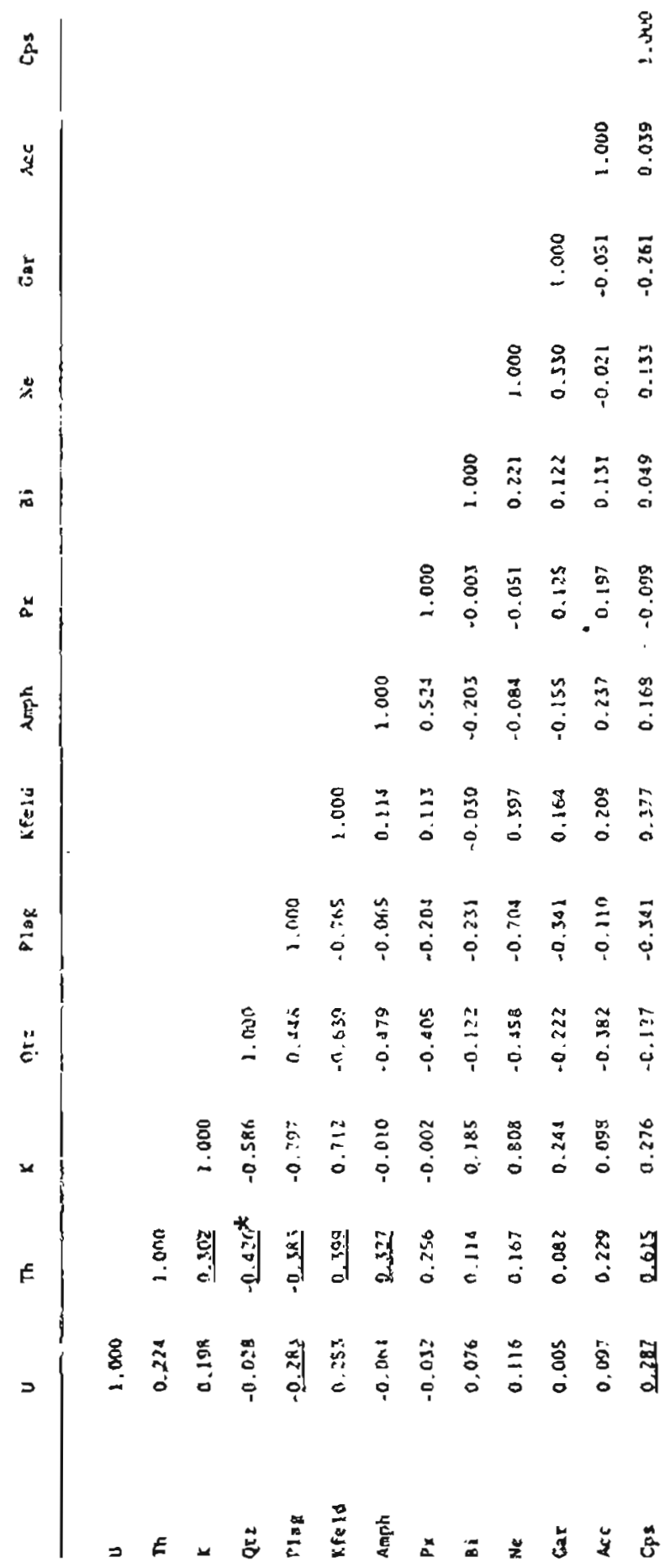

figure 20. Correlation matrix for 108 samples analyzed in this study. tunderlined values are significant correlations with uranium and thorium at a $90 \%$ confidence level. 
the thesis area upon which he should concentrate his investigations. We can suggest, from these data, that all of the plutons in the area, and not only the alkaline varieties, should be studied in some detail.

We can look at the uranium and thorium data in a different way by considering the means, standard deviations, and maxima and minima for each rock type as listed in Table 7.

Table 7

Mcans, Standard Deviations, and Maxima and Minima of Uranium and Thorium Data, By Rock Type

Rock Type

Nepheline Syenite
Alkali Gabbro
Syenite
Monzonite
l)uartz Monzonite
Cranite
Granodiorite
Rock Type

Mepheline Syenite

"lkali Gabbro

Syenite

Monzonite

Ouartz Monzon ite

Granite

Sranodiorite

Mean Uranium

12.83

8.77

5.87

3.03

5.47

10.38

2.19

$$
\begin{array}{r}
23.24 \\
1.37 \\
4.64 \\
1.98 \\
8.10 \\
6.23 \\
1.14
\end{array}
$$

32.0

1.0

7.3

1.6

17.2

8.4

0.8

49.0

19.1

4.8
$-0.3$

5.1

0.7

Thor ium

Obiervations

Mean Std. Dev. Maximum Minimum

$\begin{array}{lcccr}46.15 & 19.9 & 82.3 & 3.33 & 25 \\ 66.3 & 8.97 & 76.0 & 53.3 & 3 \\ 42.01 & 14.06 & 62.5 & 24.5 & 10 \\ 40.94 & 21.45 & 74.0 & 3.11 & 19 \\ 28.71 & 16.65 & 64.3 & 8.85 & 36 \\ 32.3 & 13.3 & 51.0 & 21.3 & 4 \\ 17.5 & 12.38 & 44.8 & 2.1 & 14\end{array}$

An analysis of variance was performed on these data to determine if there is a significant difference between the means of the individual groups. The results of this analysis are listed in Table 8.

The analysis determined that at $\alpha=.05$ there is no significant dif.. ference in the means of the uranium values. The $F$ value $=1.78$, whereas the critical $f$ at this confidence level is 2.20 .

In accordance with the observation made earlier, that there were significant correlations between thorium and mineralogy, the analysis of variance determined that there is a very significant difference between the mean thorium concentrations of individual rock types. This reflects the more predictable behavior of thorium during the differentiation process. Calculated $F$ at a $35 \%$ confidence level is 7.72 , with the critical $F$ remaining the same value as above, 2.20 . 
Table 8

Summary Table for the Analysis of Variance-Uranium

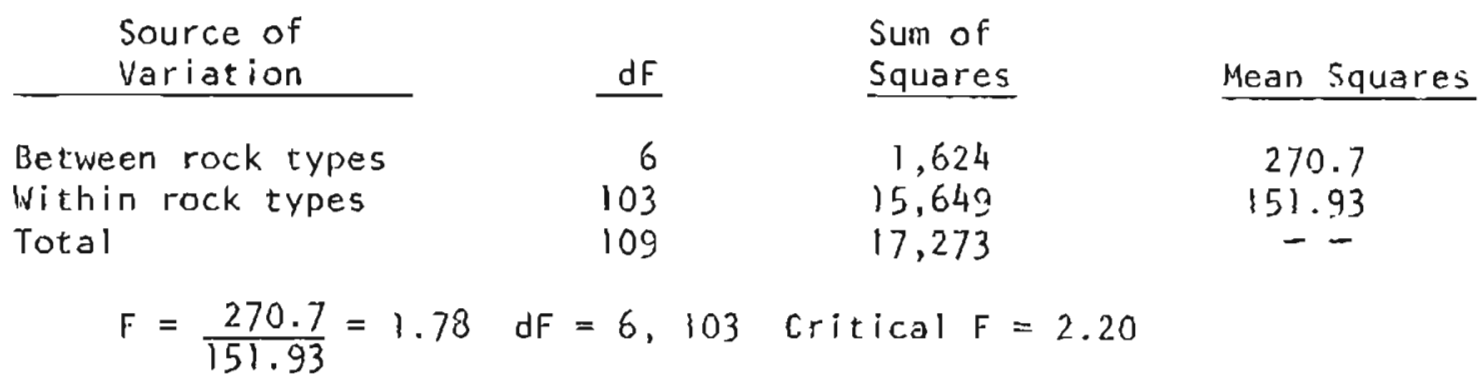

Summary Table for the Analysis of Variance-Thorium

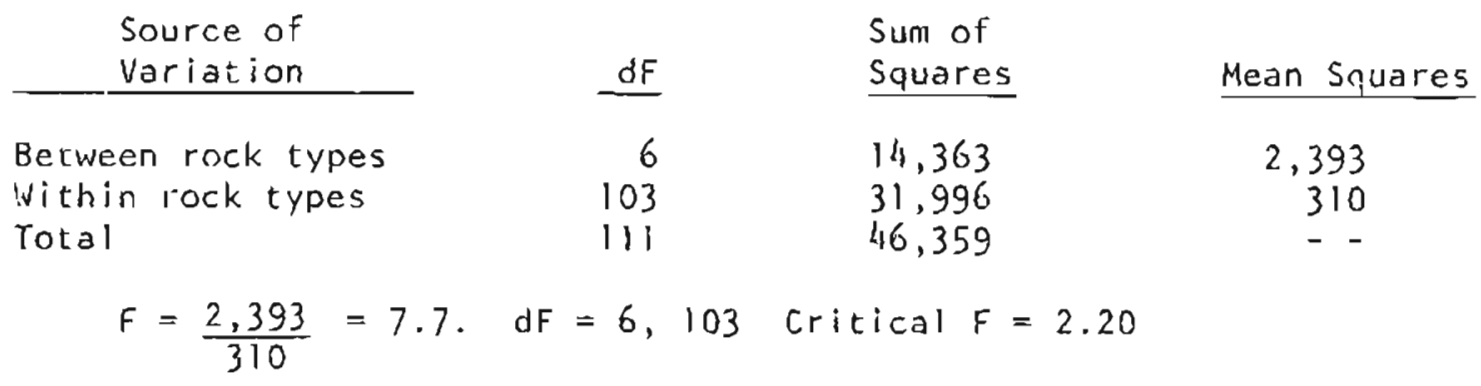

The Scintillation Counter as a Prospecting Tool

The hand-held scintillometer is standard prospecting equipment for uranlum. We can comment on the reliability of this device for recording uranium values by computing the correlation coefflcients (Figure 20 .

The correlation between uranium and counts per second is 0.287 . The correlation coefficient for thorium and counts per second is 0.615 , which is highly sigrificant. In most cases the scintillometer does not reflect the uranium content of the rock. Thorium is much more abundant in most of the rocks and the gamma radiation produced by the uranium is overshadowed by that produced from thorium. The scintillometer fails to register minor fluctuations in uranium content in an area predominantly enriched in thorium. These minor fluctuations are of little interest to the exploration geologist.

The standard deviation of an individual scintillometer reading is equal to $\sqrt{n}$, where $n=$ counting rate. The square root of 450 is 21 . Therefore the counting rate of 500 deviates significantly from 450 at the $95 \%$ confidence level. 475 is not significant at this confidence level. Therefore a considerable amount of variability in the counting rate can be attributed to statistical variation within the counter.

The mean and standard deviation for all the scintillometer data $=414$ CPS and 168 CPS, respectively. A reading greater than 582 is significant at the $1 \sigma$ level, and a reading greater than 750 is significant at the $2 \sigma$ 
level. Individual scintillometer readings are listed in Appendix 1. The only readings that exceed the 2 alpha limit are from the uraniferous rocks of the Selawik Lake Complex, and the radioactive rocks of the alkaline monzonite zone in the Zane Hills. When one considers individual readings in terms of their variability from the mean, it is possible to discriminate the more significant anomalies.

Admittediy the statistical information obtained is based on a relatively small sample size. Additional data in the Selawik Basin and eastern Seward Peninsula will aid in defining the overall background and variability of the scintillations.

Although there is little correlation hetween the radioactivity and mineralogy in the total sample set, examination of data for individual plutons revealed significant relationships that should aid the prospector.

\section{Granite Mountain}

Means, standard deviations, minima, and maxima for uranium and thorium analyses of 32 samples from the riranite Mountain pluton are shown in Table 5. The correlation matrix for Caranite Mountain is shown in Figure 21. rood correlations are apparent between uranium and potassium, $r=0.647$, uranium and nepheline, $r=0.742$, and uranium and garnet, $r=0.697$. The regression determines that 55\% of the variance of uranium can be explained by the single variable nepheline. $65 \%$ of the variance can be explained by the variables neptreline and thorium, and 70\% of the variance of uranium can be explained by nepheline, thorium, and pyroxene.

Thorium does not correlate as well with any of the mineralogical variables. In the regression, $22 \%$ of the variability of thorium can be described by the variance of uranium. The combination of thorium and total accessories explains $34 \%$ of the variance of $T h$. The addition of other variables does not appreciably improve the regression equation.

The variation in uranium, thorium, potassium, and CPS across the zones of the reanite Mountain intrusive is shown graphically in Figure 22 . There is virtually no correlation at all between uranium and CPS. Thorium has a distinct effect on the CPS reading, whereas potassium has no effect. It seems unlikely that the rest of the variability in CPS could be the result of the general background produced by cosmic radiation. Another possibility is that there is a great deal of within outcrop variability. Therefore the scintillations, recorded over an area of about 25 square feet, do not correlate well with individual samples, which vary a great deal across short distances. However, within outcrop variability was determined to be small at Granite Mountain (Figure 22). The poor correlation between thorium and counts per second is probably the result of a number of variables including cosmic radiation, analytical error, within outcrop variability, weathering variations, and the effect of potassium, i.e. variability in background.

\section{Darby Pluton}

The average uranium content of the Darby Pluton is 11 ppm (Miller, 1975a). Along the traverse the value was significantly lower, averaging 5.9 ppm for 22 observations (see Table 5). Thorium averages $37.1 \mathrm{ppm}$. 


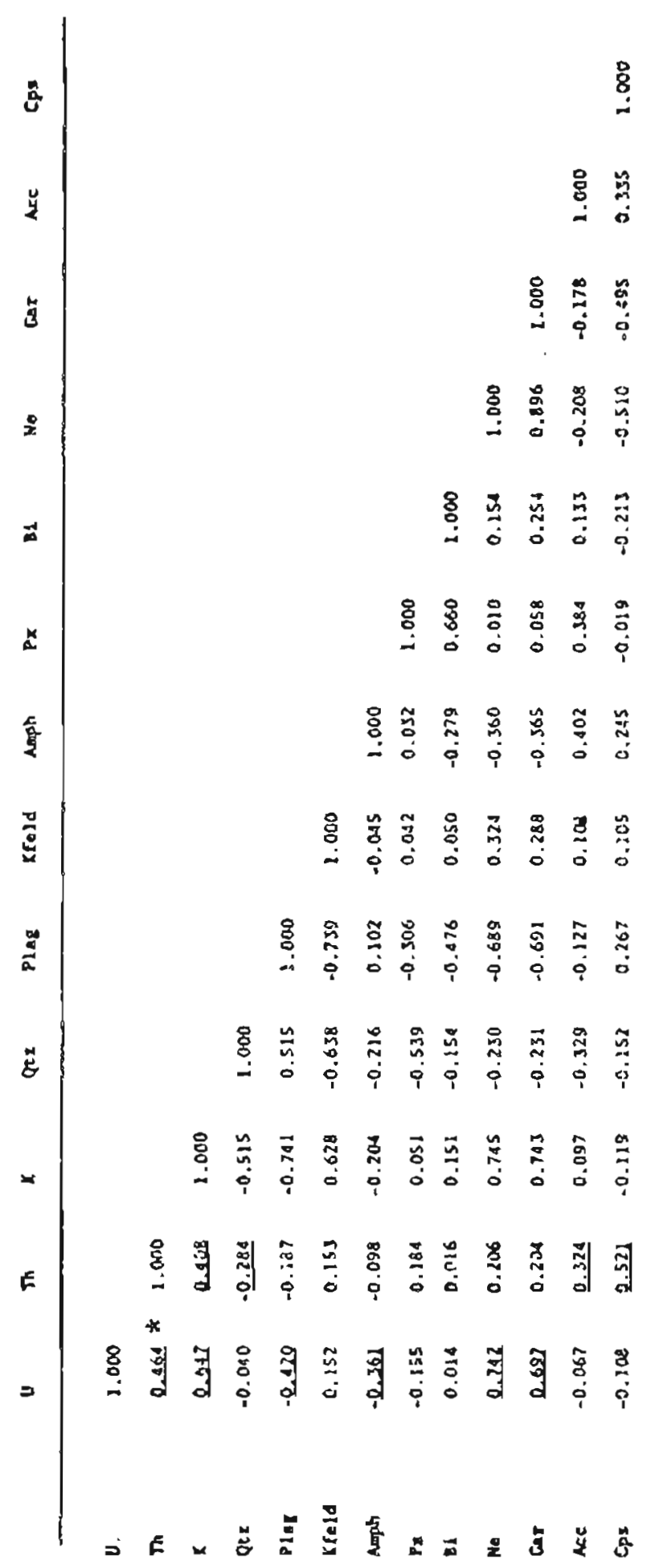

Figure 21. Correlation matrix for 32 samples from the Granite Mountain Pluton. *Underlined values are significant correlations with uranium and thorium at a $90 \%$ confidence level. 

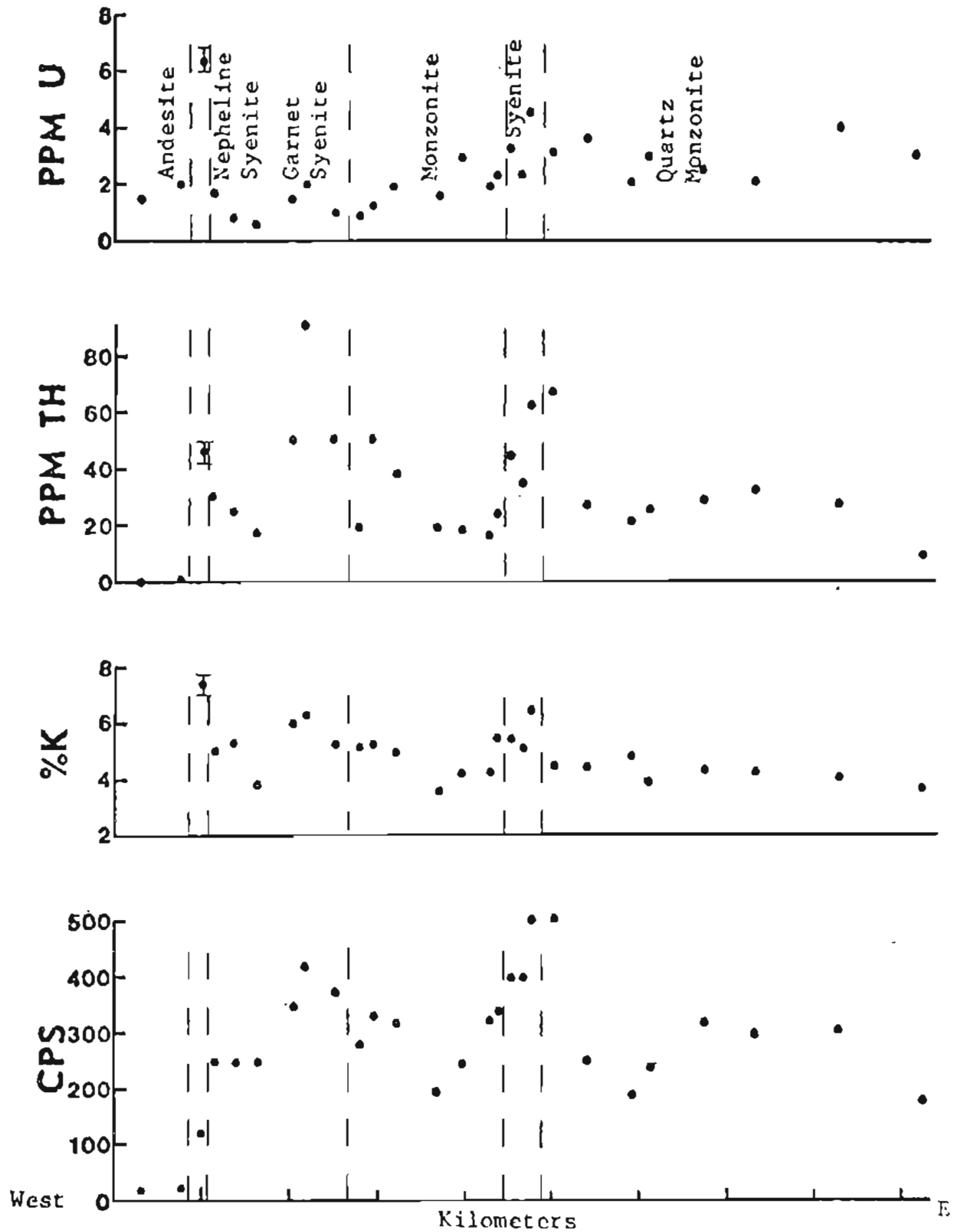

Figure 22. Variation in uranium, thorium, potassium, and counts per second across the Granite Mountain Pluton. Error bars indicate total within outcrop variability as determined from 3 samples. 
The correlation matrix for mineralogical and chemical data from the Darby Mountains is shown in Figure 23. Uranium is weakly correlative with the presence of quartz, the absence of plagioclase, and the absence of biotite. The regression shows that $33 \%$ of the variability in uranium can be described by biotite and accessory minerals. Additional variables do not improve the goodness of $\mathrm{fit}$ of the regression equation.

Thorium does not show significant correlation with any of the variables at the 90\% confidence level. In the regression, 8 variables account for on lv $35 \%$ of the variability of thorium.

Uranium, thorium, potassium, and counts per second are graphically represented in Figure 24. Data collected in the field with the use of a scintillometer suggested uranium and thorium enrichment towards the west margin of the pluton. From Figure 24 it is apparent that this enrichment does not include uranium. The increased CPS reflects a general increase in thorium. The potassium content is roughly constant across the pluton.

The number of dikes present, and the number of samples displaying the peculiar porphyritic texture described earlier, increase towards the west edge of the pluton. These observations suggest that the western edge of the Darby Pluton was an area enriched in volatiles or was more extensively fractured. The west contact of the pluton was mapped by Miller and others (1972) as an intrusive contact, whereas the eastern margin is fault bounded. Therefore the western zone represents the crystalline edge of the Darby Pluton in agreement with the expected increase ir concentration of volatiles.

Zane Mills Pluton

Means. sîandard deviations, minima, and maxima for uranium and thorium analyses from 32 samples from the Zane Hills are listed in Table 5 . The correlation natrix for the Zane Hills is shown in Figure 25. Of particular interest, are the corselations between thorium and quartz, amphibole, and $\mathrm{K}-\mathrm{feldspar}$. The relationship between thorium and quartz is shown graphically in figure 26.

Variation in quartz accounts for $62 \%$ of the variance of thorium. Quartz, and plagioclase account for $66 \%$ of the variance of thorium, and quartz, plagioclase, and amphibole account for $68 \%$. The addition of pyroxene and biotile to the regression improves the approximation of the linear fit to $72 \%$. The addition of other variables does not appreciably improve the regression equation. Figure 26 and Figure 13 show that the good correlation between quartz and thorium is not a linear relationship, but two populations; one containing little quartz and abundant thorium, and another containing abundant quartz, which is thorium-poor.

Correlation between uranium and mineralogy are not as marked. Uranium correlates with plagioclase, K-feldspar, and potassium.

The poor correlation between uranium and mineralogy is largely the result of two samples which contain high concentrations of uramium in granophyric dike rocks associated with the augen gneiss unit (Figure 27). In addition, the alkali monzonite unit, although high in thorium, does not show uranium enrichment within the samples analysed. 


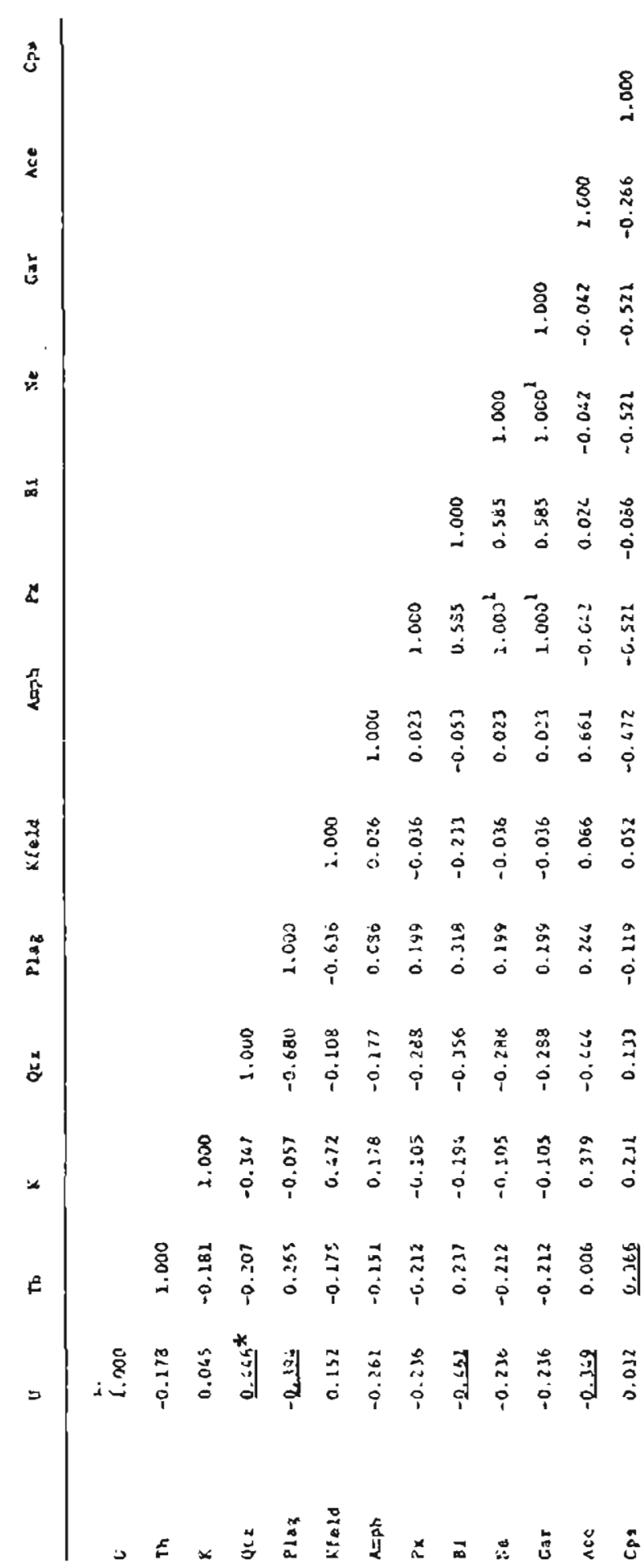

Figure 23. Correlation matrix for 22 samples from the Darby Pluton. tunderlined values are significant correlations with uranium and thorium at a $90 \%$ confidence level. 

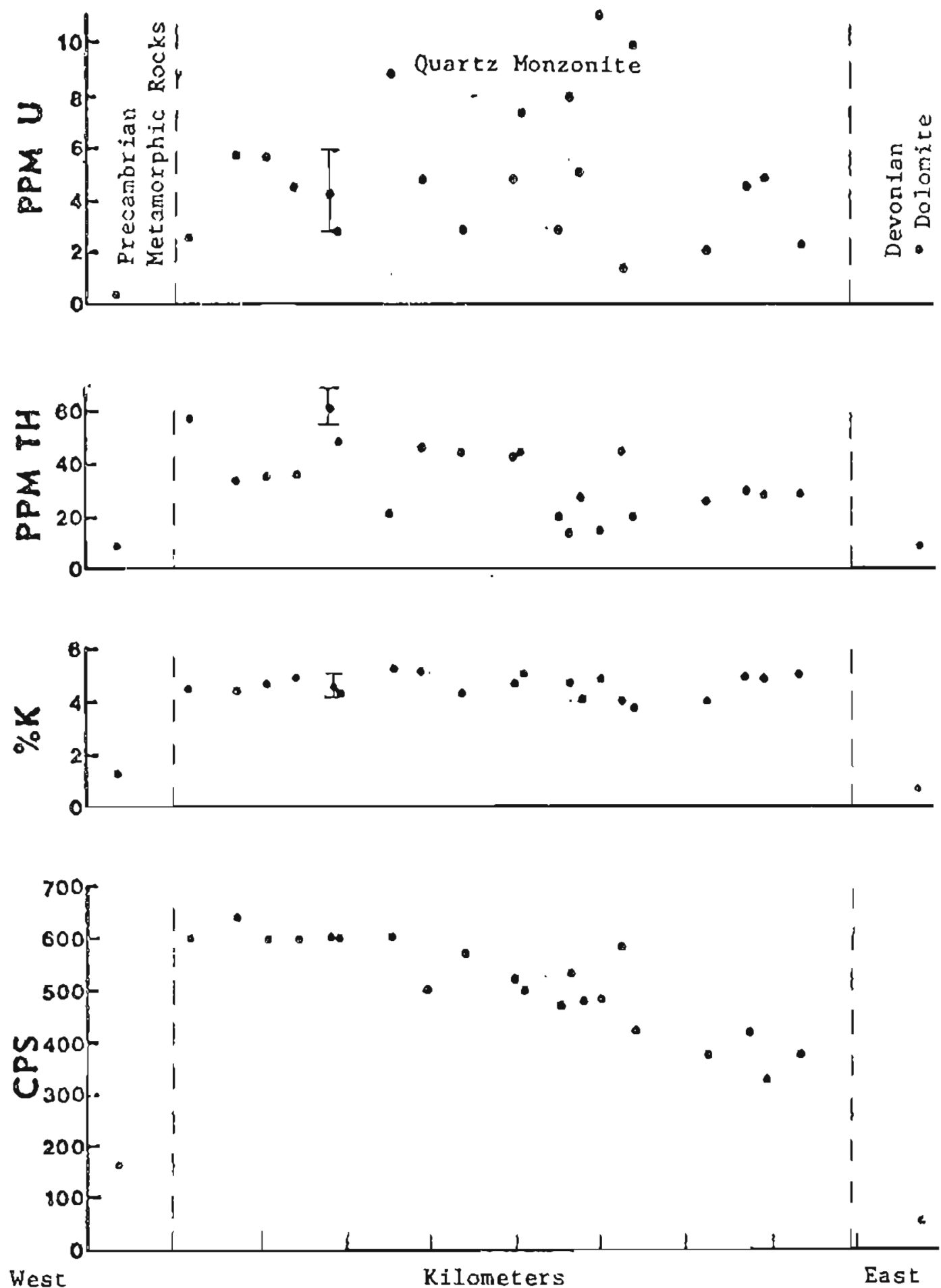

Figure 24. Variation in uranium, thorium, potassium, and counts per second across the Darby Pluton. Error bars indicate total within outcrop varialility as determined from 2 samples. 


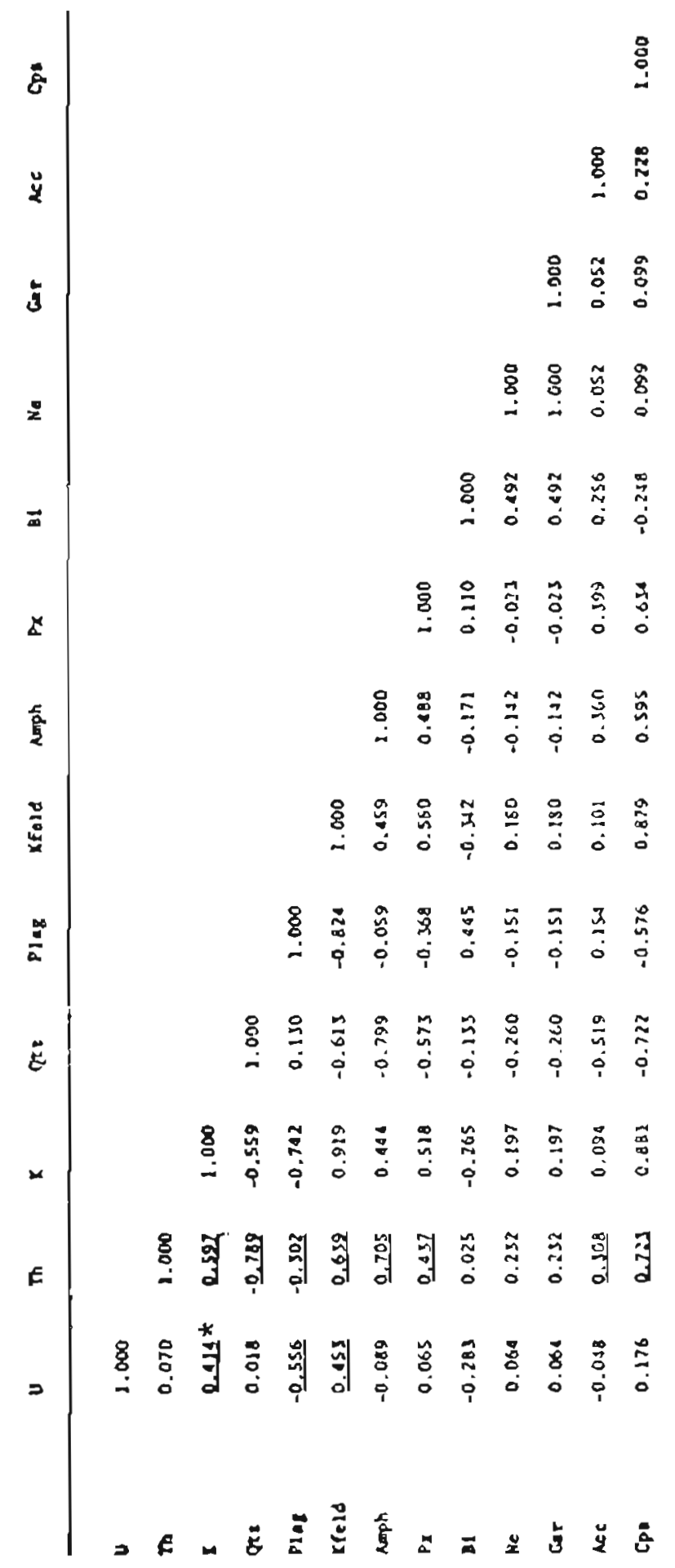

Figure 25. Correlation matrix for 32 samples from the Zane Hills Pluton. :Underlined values are significant correlations with uranium and thorium at a $90 \%$ confidence level. 


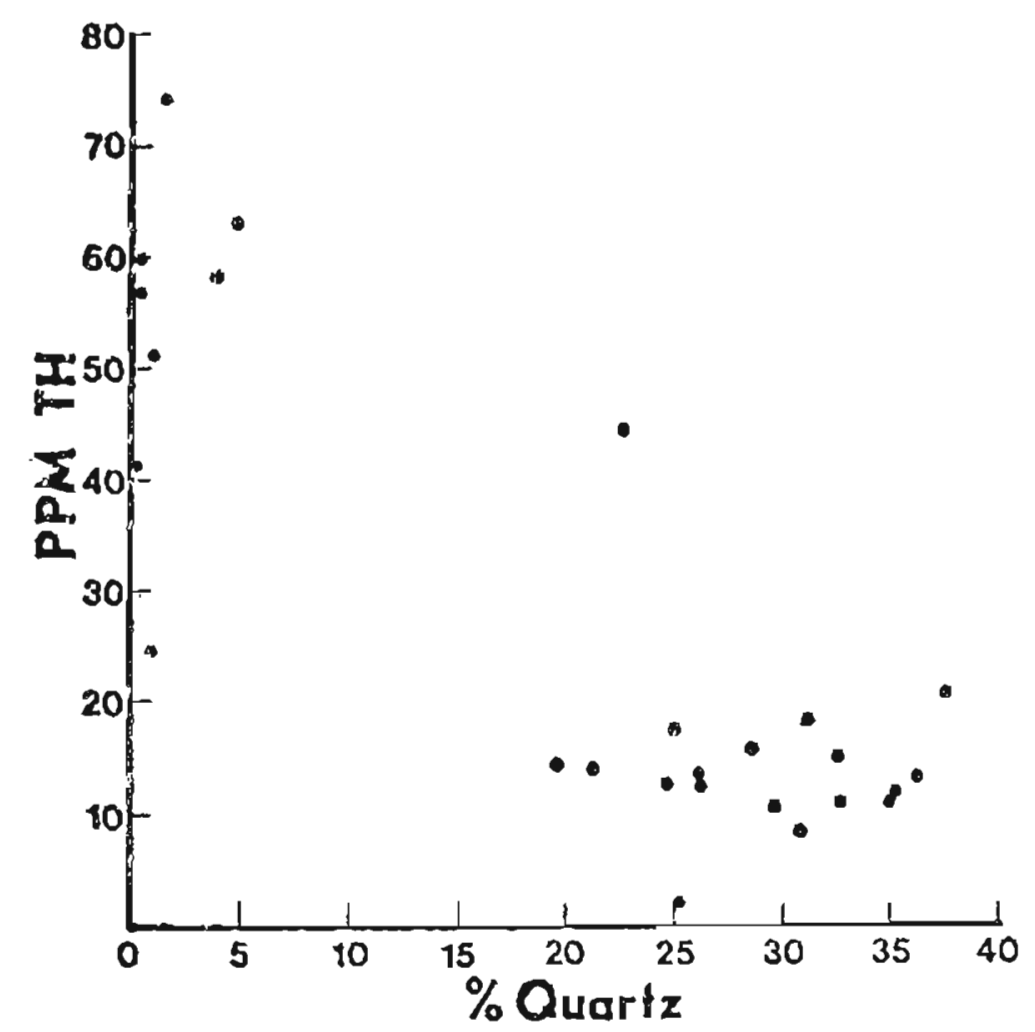

Figure 26. Plot of quartz dgainst thorium concentrations froril the Zane Hills Plutor.

Uranium, thorium, potassium, and CPS are shown graphically in Figure 27. Notice the strong correlation between thorium and potassium, and C.PS with thorium and potassium. The scintillometer closely reflects the thorium concentrations in the Zane Hills rocks. Uraniun correlates poorly with the scintillometer values. Although a few high concentrations of uranium were found, they occurred in thin dike rocks that would have little effect on a scintillometer heid at waist level. The thorium appears to be disseninated through the rock, contained in large part in minute grains of uranothorile, monazite, apatite, and sphene.

\section{The Dry Canyon Ereek Stack}

Means, standard deviations, minima, and maxima are given in Table 5 for uranium and thoriun analyses for 16 samples collecced from the Dry Canyon Creak Stock.

Thcriuni is particularly high at the Dry Canyon Creek Stock. Uranium is slightly anomalous. Scintillometer readings from this stock were quite high, up to $800 \mathrm{CPJ}$.

The correlation matrix for the Dry Canyon Creek Stock is shown in Figure 28. Uranium correlates well with the percent accessory minerals. Uraniur is largely concentrated in the accessory minerals. Thorium does not show this same relationship. A) though the data are not conclusive, it is possible that the thurium is concentrated predominantly in other mineral phases, or that the uranium originally located in the more abundani and more easily weathered rock-forming minerals, has been leached. The more resistant thorium compounds in the rock-forming minerals would remain behind. 

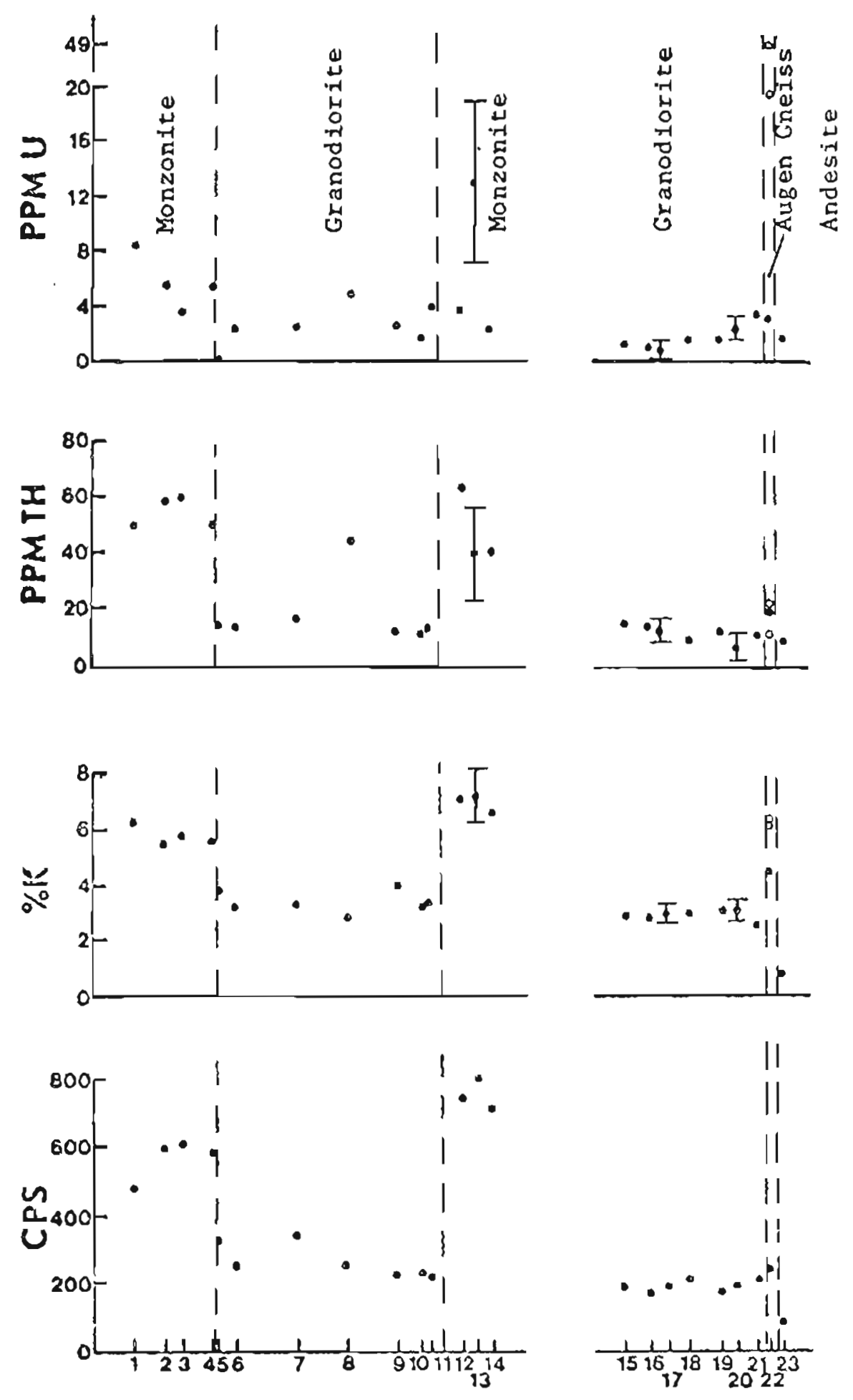

Figure 27. Variation in uranium, thorium, potassium, and counts per second along two traverses in the Zane Hills pluton. Error bars indicate total within outcrop variability, from two samples. 


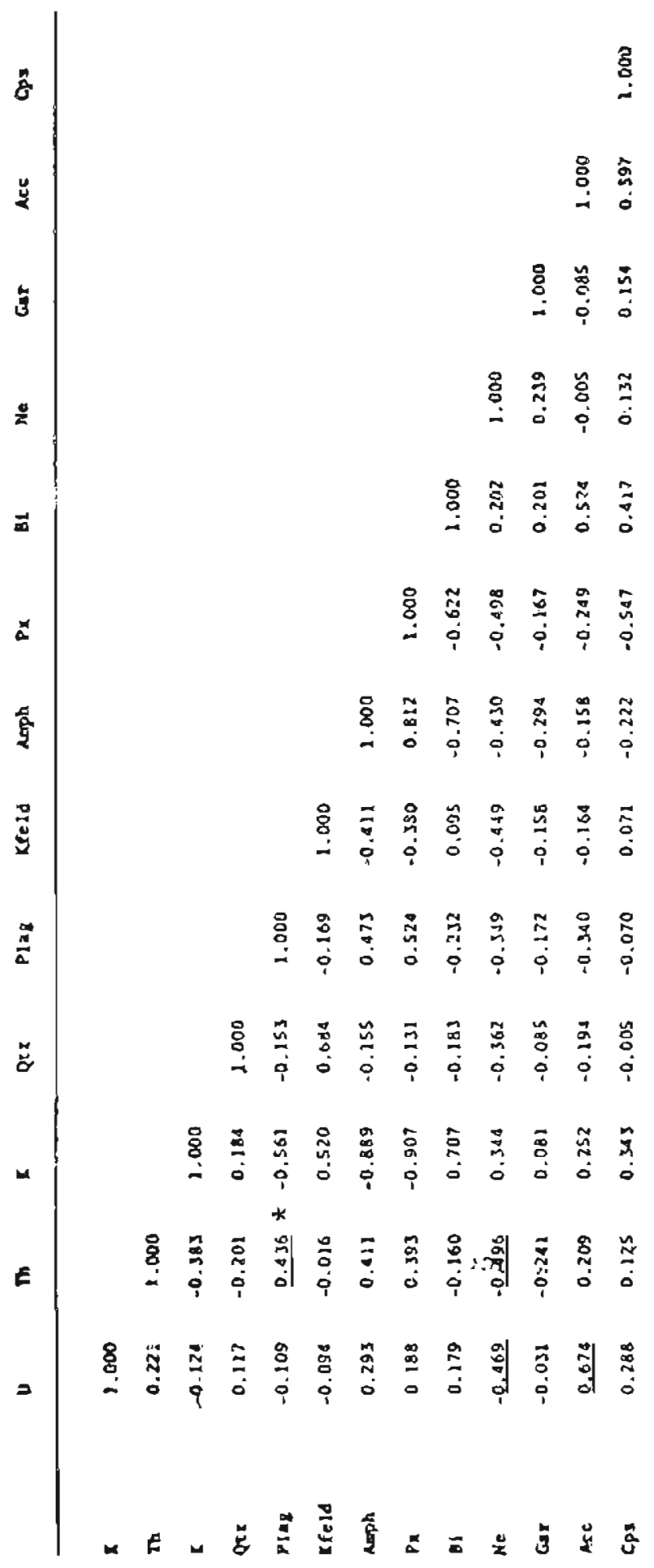

Figure 28. Correlation matrix for 16 samples from the Ory Canyon Creek Stock. xunderlined values are significant correlations with uranium and chorium at a $90 \%$ confidence level. 
Both uranium and thorium show a moderate negative correlation with nepheline. The distribution of uranium and thorium during crystallization of a magma usually shows a correlation between increase in alkalies and increase in radioactivity. Perhaps the relationship between nepheline and uranium can be explained by the oxidation of $y^{4+}$ during the latest stage of crystallization of the stock. This would remove the uranium from the intrusive into hydrothermal solutions, during the period when nepheline was crystallizing.

In the regression analysis, we are able to explain $45 \%$ of the variance of uranium in terms of the percent of accessories. Sixty-seven percent of the variance is explained in terms of accessories and nepheline, 72\% in terms of accessories, nepheline, and amphibole. The addition of thorium explains $79 \%$ of the variance, and finally the addition of quartz explains $85 \%$ of the variance. Additional variables do not appreciably improve the regression equation.

Uranium concentrations in the Dry Canyon Creek Stock can be explained quite wel! in terms of five variables, by far the most significant of which is the \% of total accessories. Uranium appears to be substituting for $\mathrm{Ca}^{2+}$ in the accessory minerals sphene and apatite.

The relationships between thorium and the mineralogical variabies are not as clear. Nepheline and quartz (two mutually exclusive variables) account for $141 \%$ of the total variance of thorium.

The scintillometer readings do not vary a great deal at the Dry Canyon creek stock, and it is not surprising that there is almost no correlation among uranium, thorium and total counts per second.

\section{Selawik Lake Complex}

Means, standard deviations, and minima and maxima for uranium and thorium anaiyses from 10 samples from the Selawik Lake Complex are shown in Table 5 .

The correlation matrix for 13 variables is shown in Figure 29 . Uranium correlates rather strongly with biotite, thorium, and counts per second. The correlation with the scintillometer reading is a result of much higher concentrations of uranium, which allow the gamma radiation emitted from the uranium to exceed that of thorium and cause a noticeable fluctuation in the counting rate. In addition uranium and thorium are correlative, and the gamma radiation that they emit is compounded in the scintillometer reading. The combination of gamma ray emissions from both elements reduces the effect of cosmic radiation and the correlations with the scintillometer are much improved.

The mineralized zone in the Selawik Lake complex is not confined to a dike rock but extends into the country rock for a few meters on either side of the intruded area. This broad concentration of uranium allows for a larger area of radioactive rock to affect the counter. This is distinctively different than the Zane Hills occurrence where the high concentrations of uranium were restricted to thin dike rocks. 


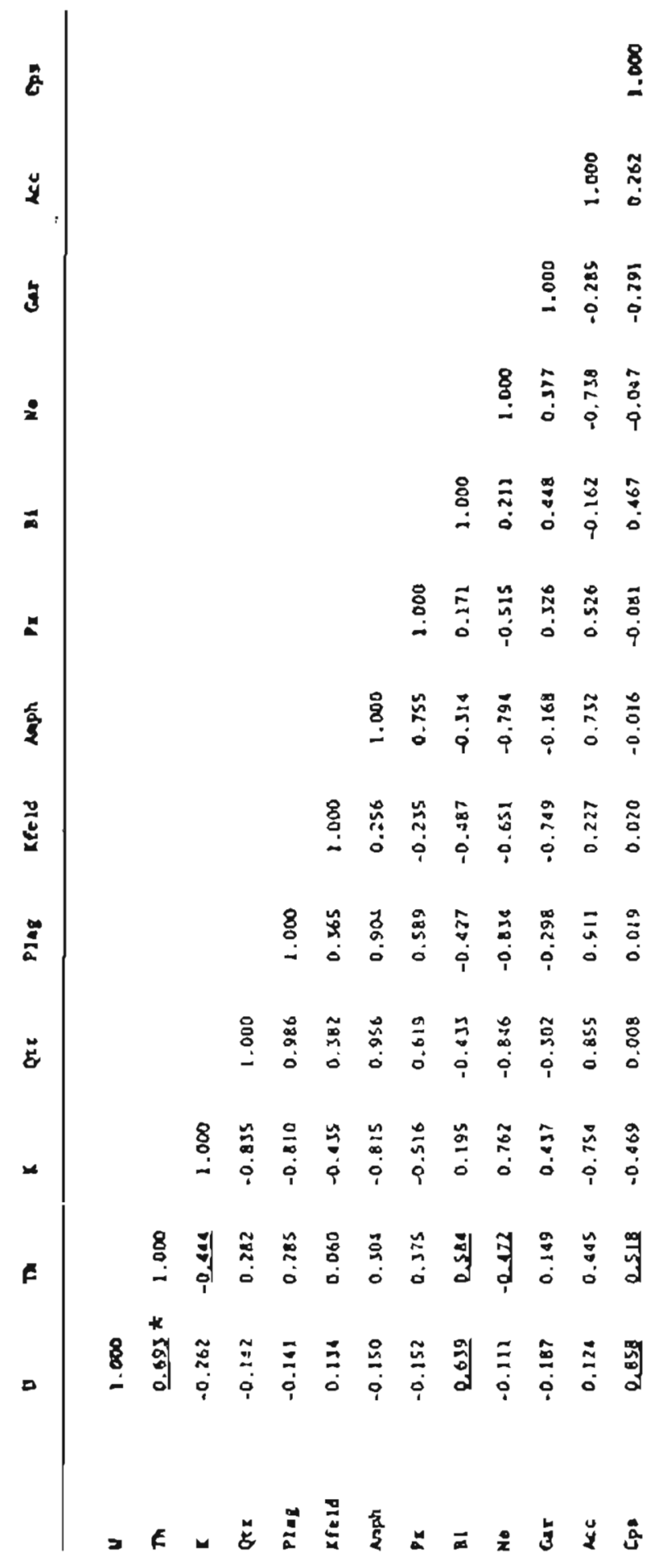

Figure 29. Correlation matrix for 10 samples from the Selawik Lake Complex. :Underlined values are significant correlations with uranium and thorium at a $90 \%$ confidence level. 
Approximately $41 \%$ of the variance of uranium is explained by variations in the biotite content. The addition of garnet to the equation increases the $R^{2}$ value to 0.69 . Ninety-three percent of the variance of uranium can be described by the variables potassium, garnet, biotite, $\mathrm{K}$-feldspar, and amphibole. For this regression, the thorium variable (because it could not be determined in the field) was not included.

We can describe the distribution of uranium sufficiently in terms of four mineralogical variables and potassium.

Seventy-two percent of the variance of thorium is explained by the variation in biotite and nepheline in the regression equation. There are no other significant variables with the exception of uraniun which was not included in the regression.

Certalniy the distribution of the radioactive elements in the Selawik Lake Complex follows a much more systematic distribution than is observed in other plutons in this study.

\section{CONCLUSIONS}

The calculation of correlation coefficients and the step-wise multiple linear regression breaks down a very complicated data set into a more concise form. The apparent result of the step-wise analysis for all of the samples within the thesis area, was that there was virtually no correlation between mineralogy, and the uranium and thorium concentrations. This is a misleading conclusion. The analysis of individual plutons shows that there are strong correlations between mineralogy and radioactivity, but that the independent variable that describes the greatest amount of variance of uranium or thorium changes from one pluzon to the next. Therefore when one considers the entire picture, the correlations in the individual plutons cancel each other and we are left with the apparently random distribution of uranium and thorium within the province.

The correlations suggest that there is some relationship between the crystallization history of the rocks and the distribution of uranium and thorium within individual plutons. The lack of correlation on a regional scale is indicative of multiple origins for the plutons. This is not surprisirg, because the age of the intrusives span a period of 20 million years, and they vary in composition from highly oversaturated granodiorite and quartz monzonite to highly alkaline nepheline syenite.

The results of this investigation are in some ways discouraging for the exploration geologist who hopes to discover a uranium ore body based on regional mineralogical associations. Had there been a strong correlation on the broad scale between radioactivity and certain rock types, perhaps the area of exploration in the Yukon-Koyukuk Basin and eastern Seward Peninsula could be greatly reduced, and individual target areas selected. The broad variation of rock type in which high concentrations of uranium are found does not allow the exploration geologist to leave any plutons unexplored in this area. 
The poor correlation between uranium and counts per second re" gistered on the hand-held scintillometer is not as discouraging a il may seem. When high concentrations of uranium are present, as in the vein deposits and associated mineralized zones of the Selawik Lake Complex, scintillometer counts represent the concentrations of uranium very well. It fails to register the small granophyre dikes of the Zane hills, but the fact that they are snall makes them a much less significant deposit than the Selawik Lake occurrence. What the scintillometer fails to record, appears to be minor variations in uranium concentration in rocks that are predominantly thoriummich. These minor variarions $\cdots$ in is orimary interest to economic geo:n $\varsigma_{1}= \pm$, gic , resiefore the failure of lha scintillometer to register them is not a hindrance to exploration.

Uranium prospecting is in a state of infancy in Mlaska when compared to the rest of the United States. The discovery of uranium concentrations up to $92 \mathrm{ppm}$ in the Selawik Lake Complex and additional high concentralion'i in a mineralized granophyric dike zone in the Zane Hills encourages tha identification of this area as a uranium and thorium province. The existence of high concentrations of the radioactive elements in accessory minerals, such as sphene and apatite, where they can be leached by weathering processes, makes possible the deposition of large quantities of uranium in the Tertiary basins believed to exist adjacent to the investigated plutons.

Recent work by the 5rate of Alaska Geological and freophysicul surusy, in cooperation with the Los Alamos laboratory indicates that the concentration of uranium in surface water samples is exceedingly low. Although this is discouraging, the paleo-environments of Alaska have changed significantly through the Cenozoic Era, since the emplacement of these plutons during Cretaceous time. Colorado-Piateau type conditions have been described from cther localities inroughout the world, lying at relatively righ latitudes (Meyerhoff, 1972). It is a possibility that the appropriate environment for leaching, distribution and precinitation of uranium was present in the Yukon-Koyukuk Basin and the eastern Seward Peninsula at some time since the emplacement of the Cretrceous flutons.

\section{Mineralogical Guidelines for Future Uranium Investigations}

\section{Granite Mountain}

Concentrate investigations on rocks containing abundant nephelire, and garnet, and littie plagioclase and amphibole.

\section{Darby Mountains}

Explore the west margin of the Darby Pluton. It appears to be a zone of abundant dike intrusion and is a likely area for uranium mineralization.

Z̈ane Hills

Concentrate investigations within the Augen fneiss Unit, and determine if it is continuous along the east margin of the pluton. Within the Monzonite unit select samples that contain little plagioclase.

\section{Dry Canyon Creek Stock}

Select samples that contain abundant accessory minerals, and that contain little nepheline. 
Selawik Lake Complex

Select samples containing abundant biotite. Use the scintillometer to outline target areas. Because of the association of biotite with uranium, magnetometer surveys of the pluton should be a useful exploration tool.

\section{REFERENCES}

Adams, J.A.S., 1954, Uranium and thorium contents of volcanic rocks, in Faul, H., ed., Nuclear Geology, John Wiley \& Sons, Inc., New York, $41 l_{1} p$.

Adler, Hans, H., 1975, Chemical factors contributing to uranium concentration in alkalic igneous rocks: Prepared for Technical Committee Meeting on Recognition and Evaluation of Uraniferous areas, Vienna, Austria, November 17-21, $13 \mathrm{p}$.

Bowen, N.L., 1928, The evolution of the igneous rocks: Princeton, Princeton Univ. Press (reprint by Dover Publications, Inc., 1956) $332 \mathrm{p}$.

Chayes, F., 1956, Petrographic modal analysis, an elementary statistical appraisal, John Wiley and Sons, Inc., Hew York, $113 \mathrm{p}$.

Davis, I.C., 1973, Statistics and data analysis in geology: John Wiley $\varepsilon$ Sons, Inc., New York, 550 p.

Deer, W.A., Howie, R.A., and Zussman, 3., 1963, Rock-forming minerals: William Clowes \& Sons, Ltd., London, 1788 p., V. 1-5.

Eakin, H.M., 1916, The Yukon-Koyukuk region, Alaska: U.S. Geol. Survey Bull. $631,88 \mathrm{p}$.

Eakins, G.R., 1975, investigation of Alaska's uranium potential: Sunmary Report for the Energy Research and Develop. Admin., ruo-1627, Part 1.

Eakins, G.R., Forbes, R.B., Jones, B.K., and Carver, C., 1976, Uranium report, contract number AT(05-1)-1639, in preparation.

Elliott, R.L., and Miller, T.P., 1969, Results of stream sediment sampling in the western Candle and southern Selawik quadrangles, Alaska: U.S. Geol. Survey Open-file report, $61 \mathrm{p}$.

Forbes, R.B., Investigation of Alaska's uranium potential: Summary Report for the Energy Research and Develop. Admin., GJ0-1627, Part 2.

Fudali, R.F., 1963, Experimental studies bearing on the origin of pseudoleucite and associated problems of alkalic rock systems: Geol. Soc. America Bull., V. 74, p. 1101-1126.

Gault, H.R., Killeen, P.L., and West, W.S., 1953, Reconnaissance for radioactive deposits in the northeastern part of the Seward Peninsula, Alaska, 1945-47 and 1951: U.S. Geol. Survey Circ. 250, 31 p.

Honeywell Time-sharing Applications Library Guide, V. II Statistics, 1973: Honeywell Information Inc., Wellesley Hills, Mass., 225 p.

Kleeman, J.0., and Lovering, J.F., 1967, Uranium distribution studies by fission track registration in lexan plastic prints: Reprinted from the AAEC Journal, "Atomic Energy in Australia," $8 \mathrm{p}$.

Korkisch, J. and Dimitriadis, D., 1973, Anion-exchange separation and spectrophotometric determination of thorium in geological samples: Talanta, v. 20, p. 1199-1205.

Larsen, E.S., Jr., and Phair, G., 1954, The distribution of uranium and thorium in igneous rocks, in Faul, H., ed., Nuclear Geology, John Wiley $\&$ Sons, inc., New York, 414 p.

Meyerhoff, A.A., 1972, The new global tectonics: Major inconsistencies: Am. Assoc. Petroleum beologists Bull., V. 56, p. 269-336. 
Miller, T.P., Patton, W.W., Jr., and Lanphere, M.A., 1966, Preliminary report on a plutonic belt in west-central Alaska, in feological Survey Research 1966: U.S. Geol. Survey Prof. Paper 550-0, D. D158-0162.

, and Ferrians, 0.J., Jr., 1968, Suggested areas for prospecting in the central Koyukuk River region, Alaska: U.S. Geol. Survey Circ. $570,12 \mathrm{p}$.

, and Elliott, R.L., 1969, Metalliferous deposits near Granite Mountain, eastern Seward Peninsula, Alaska: U.S. Geol. Survey Circ. $614,19 \mathrm{p}$.

Miller, T.P., $1970 a$, Petrology of the plutonic rocks of west-central Alaska (Ph.D. thesis): Stanford University, Stanford, California, $132 \mathrm{p}$.

,Elliott, R.L., Grybeck, O.H., and Hudson, T.L., 1971, Results of geochemical sampling in the northern Darby Mountains; Seward Peninsula, Alaska: U.S. Geol. Survey Open-file report, $12 \mathrm{p}$.

, 1972, Potassium-rich alkaline intrusive rocks of western Alaska:

Geological Society of America Bulletin, v. 83, p. 2111-2128.

, and Bunker, C.M., 1975a, A reconnaissance study of the $U$ and $T h$ contents of plutonic rocks of the southeastern Seward Peninsula, Alaska: U.S. Geol. Survey Open-file report, $33 \mathrm{p}$.

, 19756, U, Th, and $K$ analyses of selected plutonic rocks from west-central Alaska: U.S. Geol. Survey Open File report, 5 p.

, 1976. Hardrock uranium potential in Alaska: U.S. Geol. Survey Open-file report, $14 \mathrm{p}$.

Moffitt, F.H., 1905. The Fairhaven gold placers, Seward Peninsula, Alaska: U.S. Geol, Survey Bulletin $247,85 \mathrm{p}$.

Norman, M.B., 1974, Improved techniques for selective staining of feldspar and other minerals using amaranth: Jour. Research U.S. Feol. Survey, V. 2, No. 1, p. 73-74.

Panofsky, H.A., and Brier, G.W., 1968, Some Applications of Statistics to Meteorology: The Pennsylvania State University, University Park, Pennsylvania, 22t $p$.

Patton, W.W., Jr., and Miller, T.P., 1966, Regional geologic map of the Hughes quadrangle, Alaska: U.S. Geol. Survey Misc. Geol. Inv. Map 1-459, scale $1: 250,000$.

, 1967, Regional geologic map of the Candle quadrangle, Alaska: U.S. Geol. Survey Misc. Geol. Inv. Map 1-492, scale 1:250,000.

Patton, W.W., Jr., and Miller, T.P., 1968, Regional geologic map of the Selawik and southeastern Baird Mountain quadrangles, Alaska: U.S. Geol. Survey Misc. Creol. Inv. Map 1-530, scale I:250,000.

, and Miller, T.P., and Tailleur, I.L., 1968, Regional geolouic map of the Shungnck and southern part of the Ambler River quadrangles, Alaska: U.S. Geol. Survey Misc. Geol. Inv. Map 1-554, scale 1-250,000. 1970a, Mesozoic tectonics and correlations in Yukon-Koyukuk province, west-central Alaska (abs.): Am. Assoc. Petroleum Geologists Bull., v. 54, No. 12 , p. 2500.

- 197l, Preliminary geologic investigations of western St. Lawrence Island, Alaska: U.S. Geol. Survey Prof. Paper 684-C.

Pliler, R., and Adams, J.A.S., 1962, The distribution of thorium and uranium in a Pennsylvanian weathering profile: Cieochim. et Cosmochim., Acta, v. 26, p. $1137-1146$.

Sainsbury, C.L., 1969. The A.J. Collier thrust belt of the Seward Peninsula: Cieol. Soc. America Bull., V. 80, no. 12, p. 2595-2596.

Sarantsina and Shinkarev, 1967, Petrography of Magmatic and Metamorphic Rocks (in Russian): Nedra, Leningrad. 
Shand, S.J., 1922, The problem of the alkaline rocks, Proc. reol. Soc. S. Afr. 25, $x|x-x \times x| 11$.

, 1939, On staining of feldspathoids and on zonal structure of nepheline: Am. Mineralogist, 24, 508-513.

Smith, P.S., and Eakin, H.M., 1911, A geologic reconnaissance in southeastern Seward Peninsula and the Norton Bay-Nulato region, Alaska: U.S. Geol. Survey Bull. 449, 146 p.

Sorensen, H., 1970, Low-grade uranium deposits in agpaitic nepheline syenites, South Greenland, in Uranium Exploration Geology: International Atomic Energy Agency, Vienna, Austria, p. 151-159.

, 1970, Occurrence of uranium in alkaline igneous rocks, in Uranium Exploration Geology: International Atomic Energy Agency, Vienna, Austria, P. 161-168.

, 1974, The aikaline rocks: The Garden City Press l.imited, Letchworth, Hertfordshire, Great Britain, $622 \mathrm{p}$.

Staatz, M.H., and Miller, T.P., 1976, Uranium and thorium content of radioactive phases of the Zane Hills pluton: U.S. Geol. Survey Cire. 733. P. 39-41.

Stanton, R.L., 1972, Ore petrology, Mcraw-Hill, inc., 713 p.

Texas Instruments Incorporated, 1975, Airborne geophysical survey of Copper River and Seward-Selawik areas. Alaska: Prepared for United State Energy Research and Development Administration, Grand Junction office.

Hest, W.S., and White, M.G., 1952, The occurrence of zeunerite at Brooks Mountain, Seward Peninsula, Alaska: U.5. Ceol. Survey Circ. 214, 7 p. , 1953, Reconnaissance for radioactive deposits in che Darby Mounliains, Seward Peninsula, Alaska, 1948: U.5. Reol. Survey Circ. 300, $7 \mathrm{p}$. 
0000000000000000000000000000

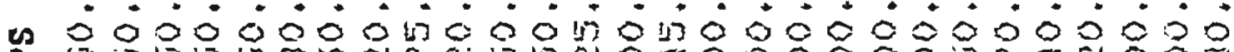
स

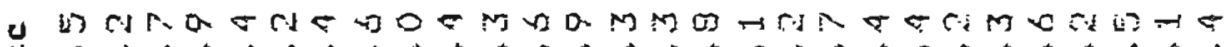

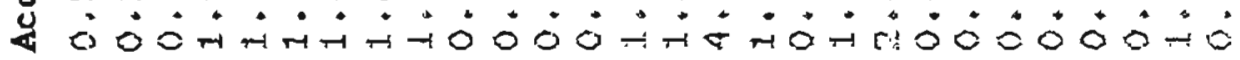
4) MnO0000000000000000,00000000 H. M 100000000000000000000000000 य

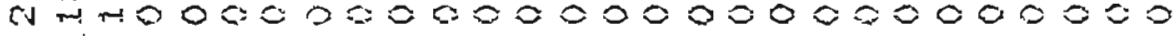

munto0000000000000000000000\%

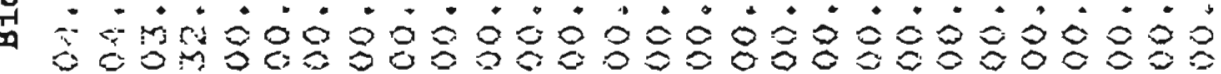
H m m m

म

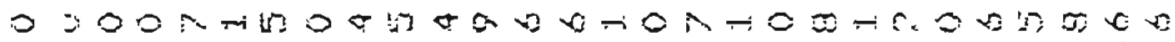

帛 A $\mathrm{d}$
$\mathrm{l}$

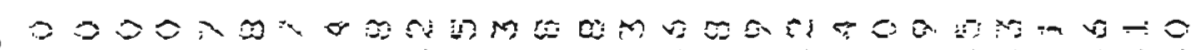

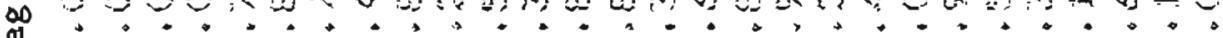

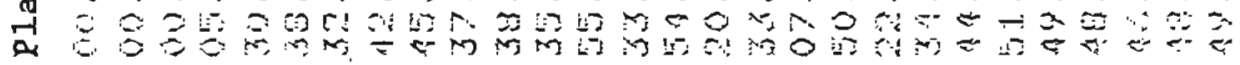

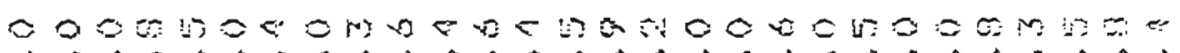
出 $\dot{0} \dot{0} \dot{0} \dot{0} \dot{0} \dot{0} \dot{0} \dot{0} \dot{0} \dot{0} \dot{0} \dot{0} \dot{0} \dot{0} \dot{0} \dot{0} \dot{0} \dot{0} \dot{0} \dot{0}$ $\sigma=00000000000000000000-1-\pi+m i$

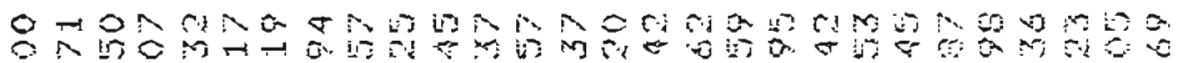

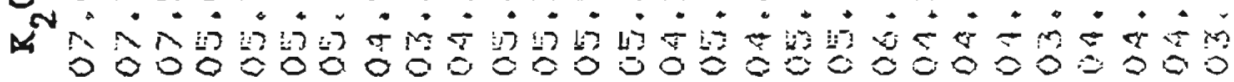

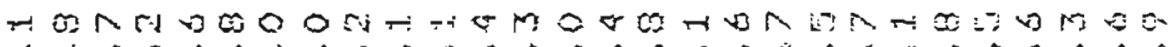

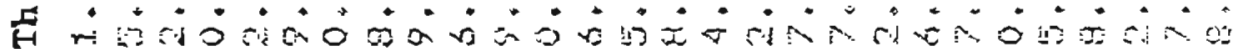

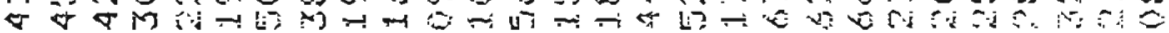

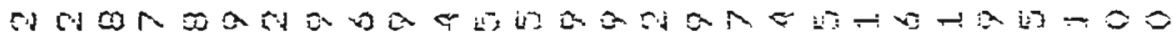

$\Rightarrow$ व

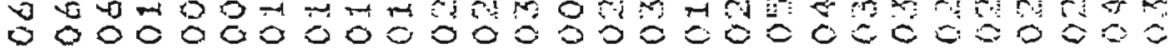

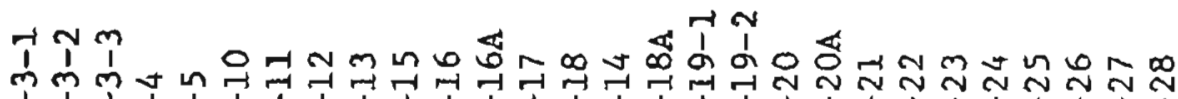

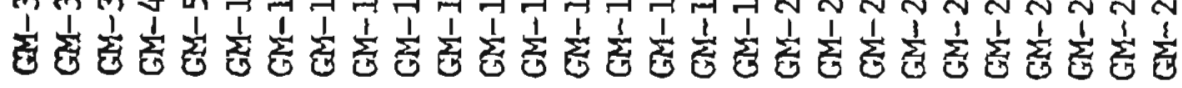
aoznTd afezunOK azfuezo 


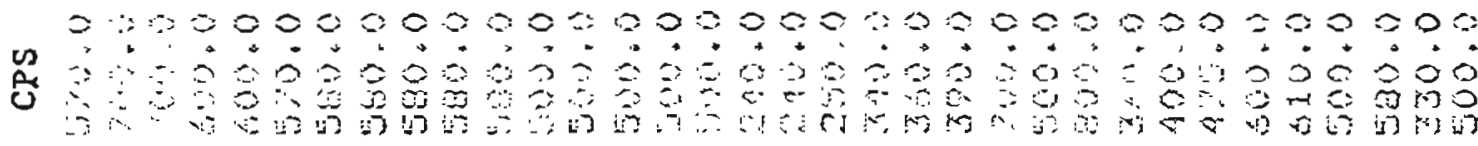

u

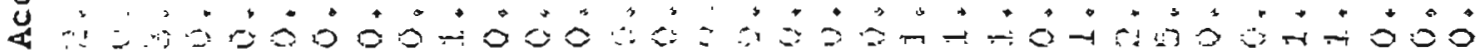

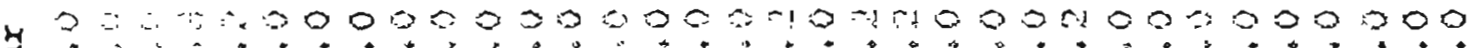

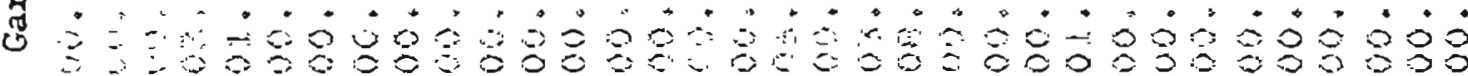

;

中 $\therefore-6 \%$ -

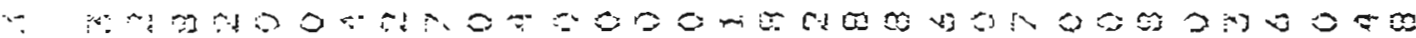

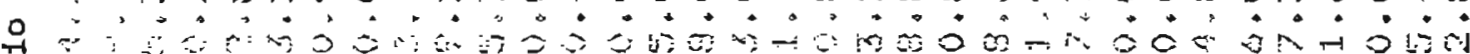

中

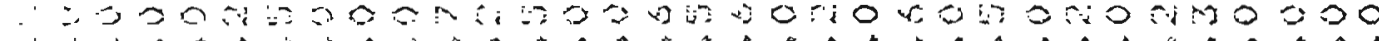

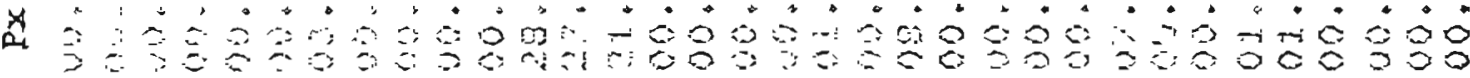

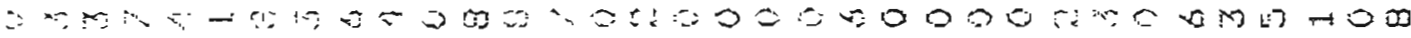

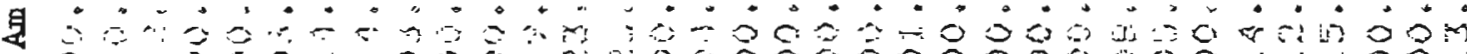
-

品

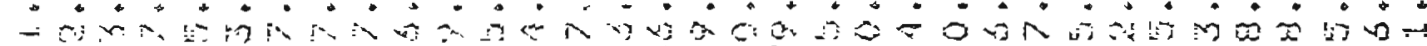

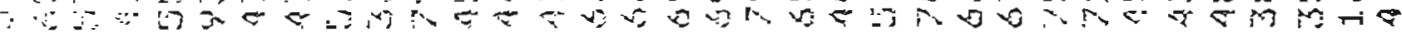

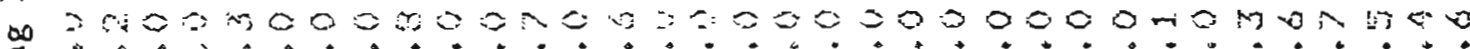
开 $00000: 000000000=000000000000$ mathor

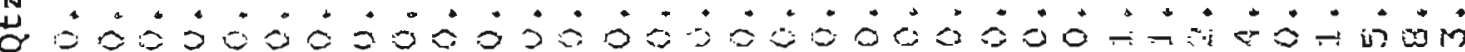

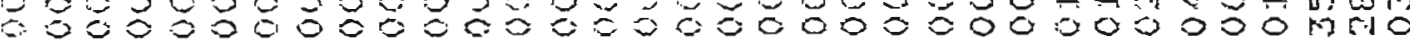
in

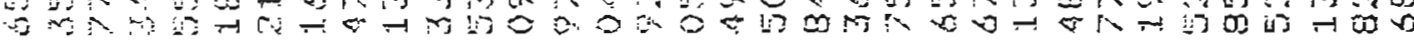

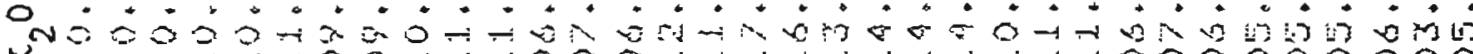

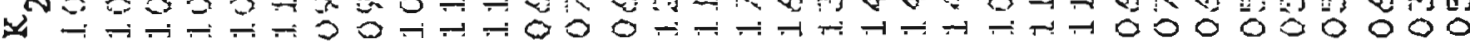

N 4 -

E करे ज:

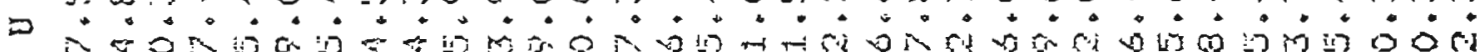

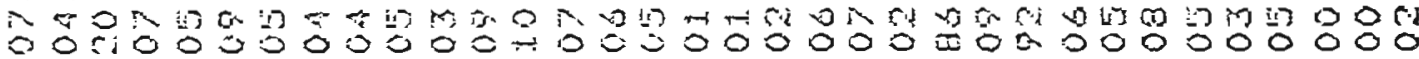

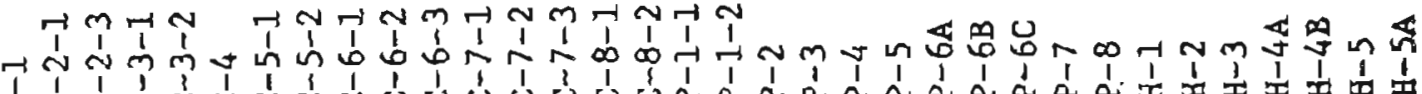

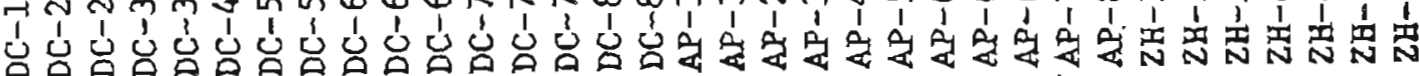


000000000000000000000000000000000

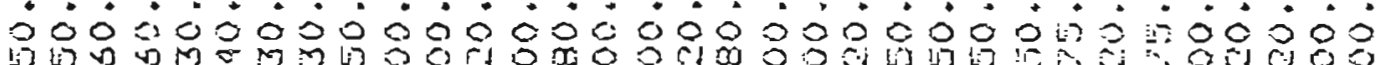

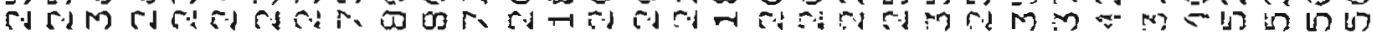

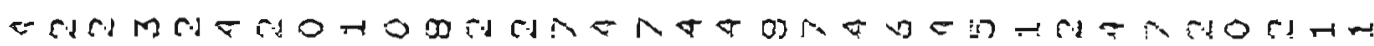

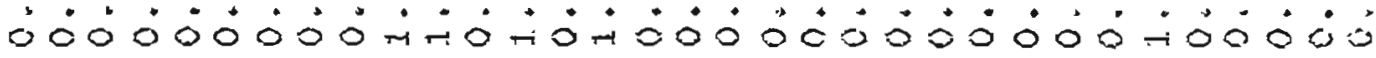
000000000000000000000000000000000

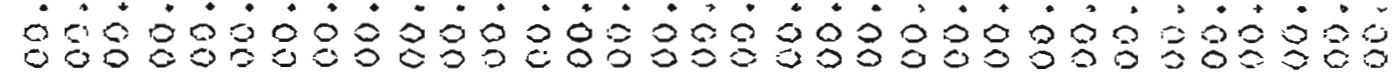
$0000000000000000 \leqslant 0000000000000000$ य

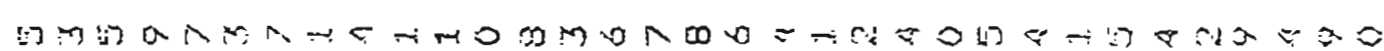

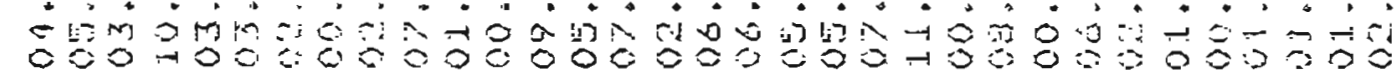
000000000 arpo0000000000000000000

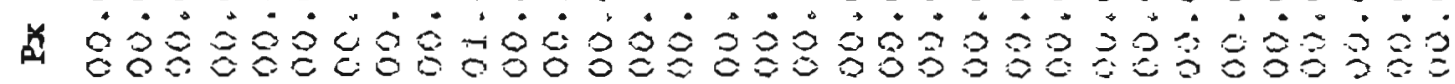
00000000 1

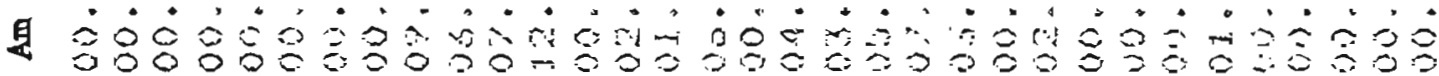

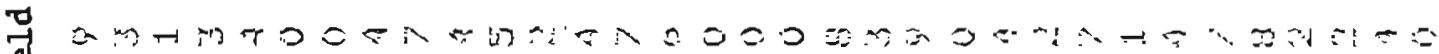

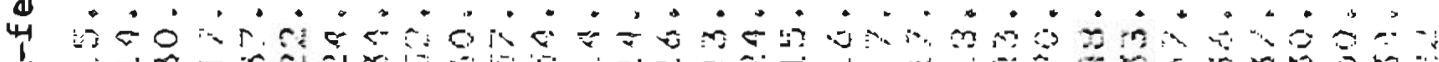

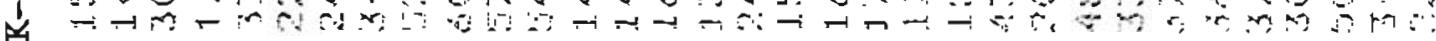

D 64

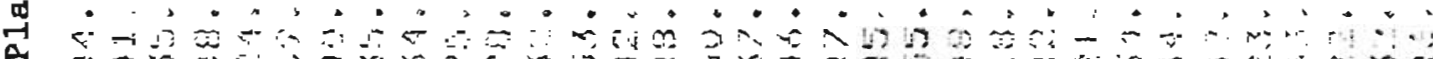
A $<$ \&

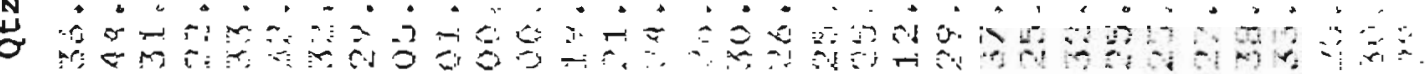

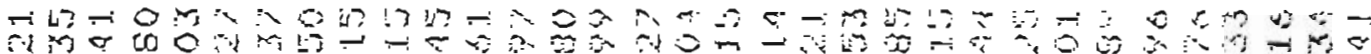
o ๙ 山!

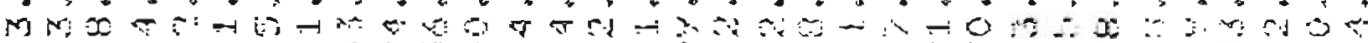

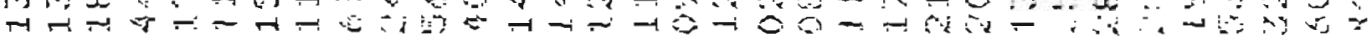

D बM

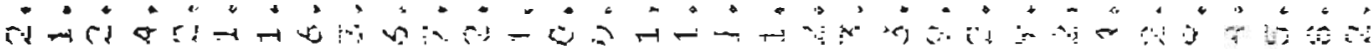
$0000000000-10005000000 \div 000000000$

\section{$\rightarrow \widetilde{N}$} i

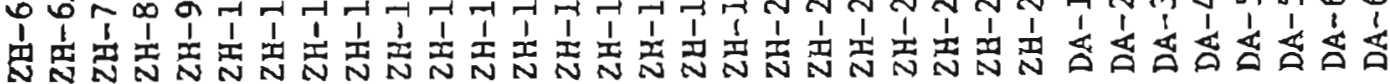


$00000<00000000$

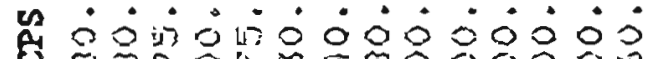

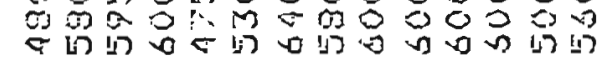

य

400000000000000

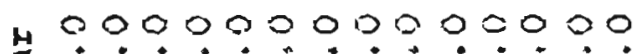

号 $\dot{0} \dot{0} \dot{0} \dot{0} \dot{0} \dot{0} \dot{0} \dot{0} \dot{0} \dot{0}$ 0050000000000

00000000000000

வ $: \dot{0} \approx \dot{0} \dot{0} \dot{0}=\dot{0} \dot{0} \dot{0} \dot{0}$ घ0 $000=00000000$

0 सा:

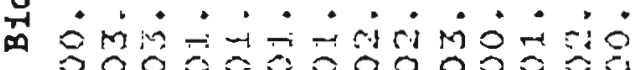

x 00000000000000 - $\dot{0} \dot{0} \dot{0} \dot{0} \dot{0} \dot{0} \dot{0} \dot{0} \dot{0} \dot{0}$

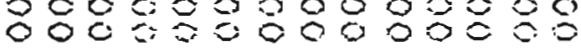

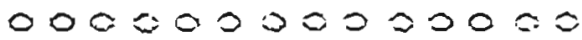

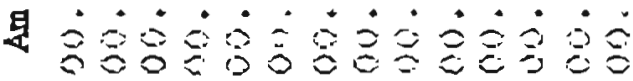

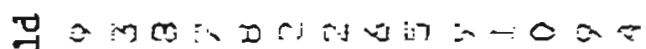

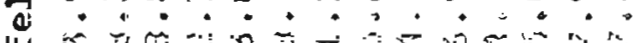

山 ?

$\infty$ แ

d $\mathrm{d}$ के

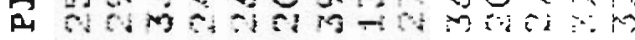

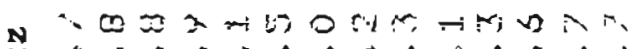

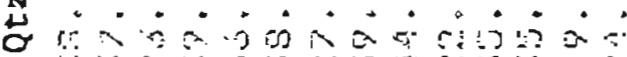

जी

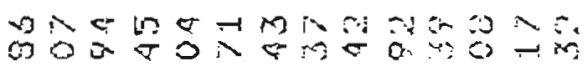

O

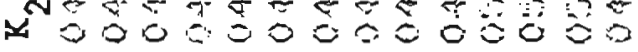

y

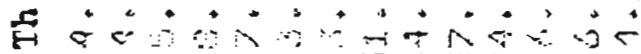

$\Rightarrow$ F

EM M

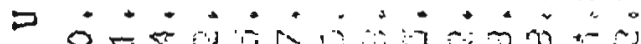

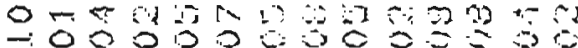

त ल ल

N

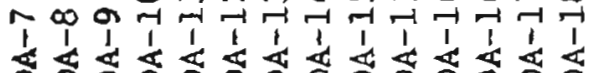

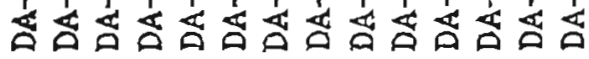
4oznTd kqIea

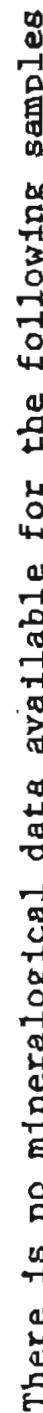

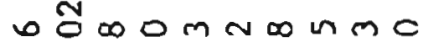
$\dot{0} \dot{0} \dot{0} \dot{0} \dot{0} \dot{0}$

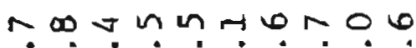

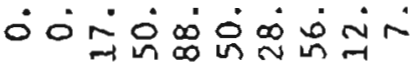

un $060000 \mathrm{~m}$ -í⿴囗十்

HNon on on 유메

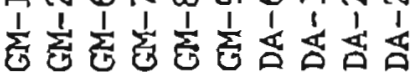




\section{Alkal ine Igneous Rocks}

The term alkaline has not been used consistently in the past. Sorensen (1974) summarizes the differing uses of this term.

1. Igneous rocks of Atlantic or Alkaline series (branch. group, facies).

2. Igneous rocks with alkali feldspar as the predominant feldspar, that is with more alkalies than average for their clans.

3. Igneous rocks containing feldspathoids.

4. Igneous rocks with an alkali-lime index less than 51.

5. Igneous rocks containing feldspathoids, and/or sodaPyroxenes and/or amphiboles.

We have accepted Shand's definition of alkaline rocks. Noting that alkalies are chiefly contained in feldspar, he suggested the following: If the ratio of alkalies to alumina, or the ratio of alkalies to silica in the bulk composition exceeded the ratio found in $k$-feldspar or albite, the rock is to be considered alkaline. 
APPINDIX 3

Examples of Correlation Coeffictents, as taken from Davis (1973).
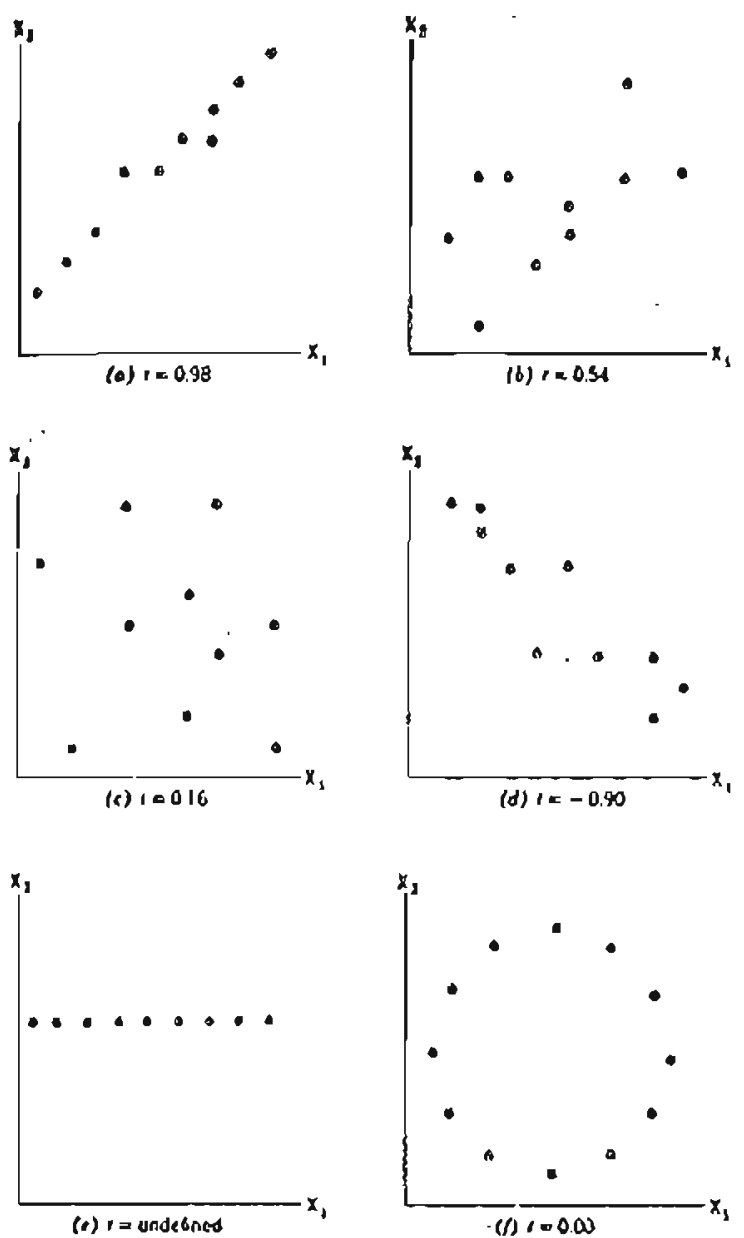

Figure 30. Correlation coefficients. 
APPENDIX 4

Analytical Techniques

Uranium Determination

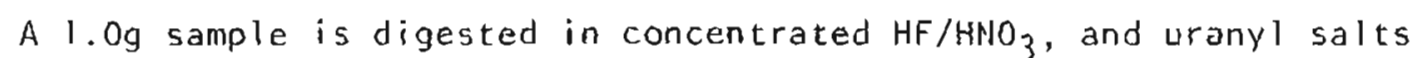
are formed. $10 \mathrm{ml}$ of this solution are extracted into ethyl acetate using a saturated aluminum nitrate solution.

$0.1 \mathrm{ml}$ of the ethyl acetate layer is extracted and placed on a mixed carbonate-fluoride pellet in a platinum crucible. The ethyl acetate is evaporated under a quartz heat lamp.

The pellets and ethyl acetate residue are then fused at a temperature of $650^{\circ} \mathrm{C}$ for exactly 15 minures.

The resulting fluorescence of the fused pellet is determined using a Turner model lis fluorometer.

Thorium Determinations (Korkisch and Dimitriadis, 1973)

Thorium is separated from all matrix elements by means of anion exchange using the strongly basic anion exchanger Dowex 1, X8 (100-200 mesh; chloride form). After elution thorium is determined spectrophotometrically by using arsenazo 111.

lon exchange separation

The sorption solution is passed through the ion-exchange coiumn containing $10 \mathrm{~g}$ of resin in the chloride form. The resin is washed, in portions, with a tutal of $100 \mathrm{ml}$ of $8 \mathrm{Mnitric}$ acid in order to remove all elements accompanying the thorium, including those which are weakly retained by the resin. The absorbed thorium is then eluted with $100 \mathrm{ml}$ of $6 \mathrm{M}$ hydrochloric acid and the eluate is evaporated to dryness on a hot plate. To the residue, $5 \mathrm{ml}$ each of $1 \mathrm{M}$ hydrochioric acid and $2 \%$ potassium permanganate solution are added and the solution is evaporated on the hot plate to destroy organic matter. The residue (which may contain manganese dioxide) is taken up in $10 \mathrm{ml}$ of concentrated hydrochloric acid and the solution is evaporated to dryness on the hot plate. Ten ml of hydrochloric acid plus 5 drops of formic acid are added 10 eliminate all nitrates and the solution is evaporated again.

\section{Arsenazo method}

The residue containing manganese (11) chloride and the thorium is dissolved in $5 \mathrm{ml}$ of concentrated hydrochloric acid and after the addition of $1 \mathrm{ml}$ of $0.2 \%$ arsenazo the solution is diluted with water to $10 \mathrm{ml}$ in a standard flask. The thorium content is obtained by measuring the resulting color of the solution using a Beckman 25 spectrophotometer.

\section{Potassium Determination}

Potassium concentrations were determined using the atomic absorption method subsequent to rusion with $\mathrm{LiBO}_{2}$. 
Reproducibility of uranium analyses

To determine the variability in the uranium analyses performed by Resource Associates of Alaska, six splits of the same sample were submitted. These results are shown below.

Table 9

Replicate Analyses of DC-5-1

$\begin{array}{cc}\text { Trial } & \text { U-ppm } \\ 1 & 5.4 \\ 2 & 6.6 \\ 3 & 5.4 \\ 4 & 6.1 \\ 5 & 7.2 \\ 6 & 5.1\end{array}$

The reproducibility for this batch of check samples was quite good. However, the results of random check samples occasionally produced drastic differences in the analysed values. Some examples are listed below:

Table 10

Replicate Analyses of Mineral Separates

Sample Irial U-ppm

Darby Mtns.

Felsic minerals

34

Biotite

$2-3 . \div$

129.

Dry Canyon Felsic minerals

$\begin{array}{lll} & 2 & 17 . \\ \text { Felsic minerals } & 1 & 19 .\end{array}$

Pyroxene 1140.

216.

Wranium concentration is below the detection limit

The above samples were mineral separates. One would expect a larger error associated with these samples. However some of the results indicate a serious technical error. The unrealistically high values are believed to have been caused by contamination of the sample from the previous analysis. This presents a problem that must be considered when working with the uranium analyses in this report. A uranium analysis succeeding a high value may sometimes show a concentration of uranium where this concentration is the result of contamination.

The highest uranium values reported in this paper were resumitted to the laboratory to check the validity of these results.

\section{Point Counting Techniques}

Modes were determined for 108 rock samples using point counting 
techniques. Nne thousand points were counted for each rock sample. Rock slabs were counted by placing a grid system, on transparent acetate, directly onto the rock slab. The counting interval was approximately equal to the largest crystal diameter, except in the highly porphyritic rocks.

Standard 1,000 $\mathrm{mm}^{2}$ thin sections were counted using a J. Swift and Sons automatic mechanical stage. Two-inch by three-inch sections, equal to $3,000 \mathrm{~mm}^{2}$ in area were counted using a hand operated mechanical stage.

According to Chayes (1956), the accuracy of the point counting analysis can be estimateo in terms of the grain size and the area of the rock that is counted. Grain size is measured in this determination as "the number of major mineral identity changes along a unit length of line". The unit length is $40 \mathrm{~mm}$. This analys is yields a number that is called the IC number.

Figure 31 shows the number of thin sections required for maintaining average major mineral analytical error $51.4 \%, 2.0 \%$ and $2.5 \%$. Based on the determination of IC numbers of a number of rock samples in this study, the analytical error is approximately $\leq 2.0 \%$.

\section{Staining Techniques}

To aid in modal determinations, rock slabs, and thin sections were stained for potassium feldspar, plagioclase feldspar, and nepheline.

Potassium feldspar was stained with sodium cobaltinitrite after etching in concentrated HF (Norman II, 1974). Rock slabs were immersed in hydrofluoric acid, whereas the thin sections were etched with HF vapor at room temperature. If there was some difficulty in discriminating plagioclase from quartz, the sample was then dipped in a saturated $8 \mathrm{aCl} 2$ solution, which gives the plagioclase a powdery white appearance. Amaranth was not used because the color produced on the etched plagioclase was quite similar to the color of quartz in many of the samples.

Nepheline was stained using methylene blue and concentrated phosphoric acid, after Shand (1939). The syrupy phosphoric acid is spread onto the thin section or rock slab using a glass rod. After about one minute the sample is gently dipped in water. A silica gel is formed as a result of the decomposition of the nepheline by the acid. When dipped in a weak methylene blue solution (0.25\%) the silica gel is stained deep blue. Preserving the stain is rather difficult. If the sample is allowed to dry the gel contracts and is easily removed in small dry flakes. A drop of watersoluble glue placed on the wet surface and covered with a coverglass will permanently preserve the stain. 


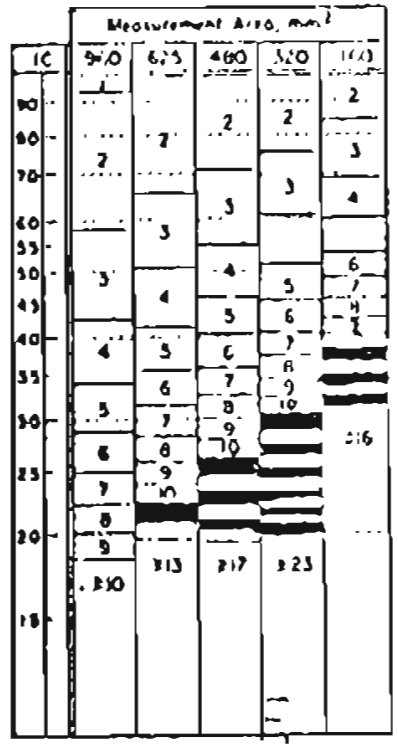

1.41

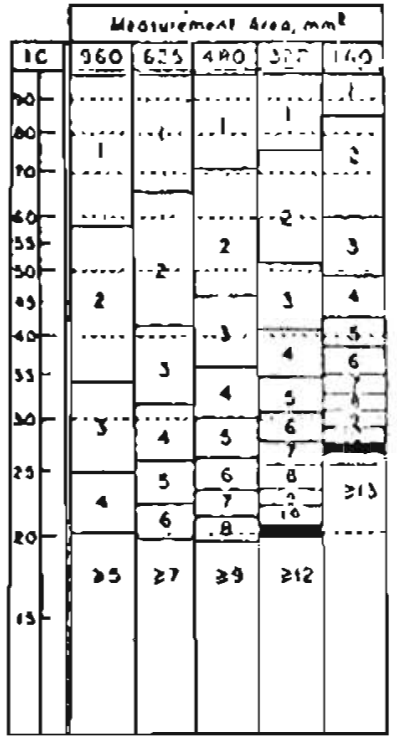

2.0

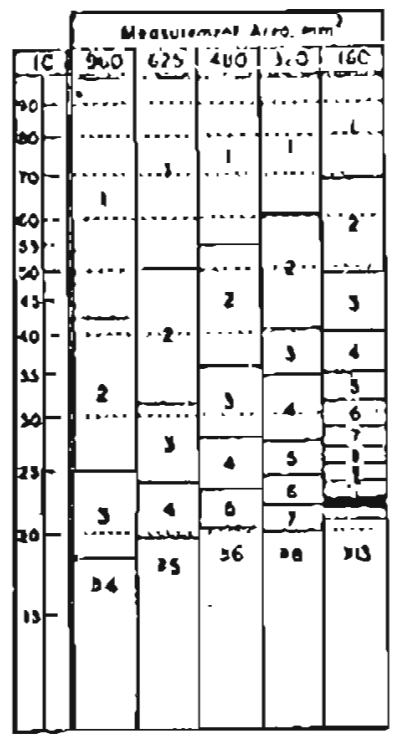

2.5

Figure 31. Number of thin sections required to maintain major mineral analytical error less than $1.41 \%, 2.0 \%$, and $2.5 \%$, based on the area of the thin section and the $I C$ number, after chayes (1956). 